\title{
Direct (Hetero)arylation of Heteroarenes Catalyzed by Unsymmetrical Pd-PEPPSI-NHC Complexes under Mild Conditions
}

A-Xiang Song, Xiao-Xiao Zeng, Bei-Bei Ma, Chang Xu, ${ }^{*}$ Feng-Shou Liu*

School of Chemistry and Chemical Engineering, Guangdong Cosmetics Engineering \& Technology Research Center, Guangdong Pharmaceutical University, Zhongshan, Guangdong, 528458, China

Contents of Supporting Information

1. NMR data of cross coupling products S2-S11

2. NMR spectrums of the ligands and palladium complexes S12-S17

3. NMR spectrums of the arylated cross-coupling products. S18-S81

4. GPC curves S81

5. Tables of reaction optimization S82 
The NMR data of compounds were obtained on a Varian Mercury-Plus $400 \mathrm{MHz}$ spectrometer at ambient temperature with the decoupled nucleus, using $\mathrm{CDCl}_{3}$ as solvent and referenced versus TMS as standard.

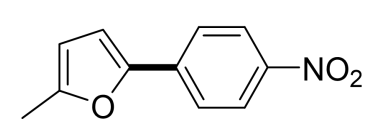

2-methyl-5-(4-nitrophenyl)furan (3aa): ${ }^{1} \mathrm{H}$ NMR $\left(400 \mathrm{MHz}, \mathrm{CDCl}_{3}\right) \delta$ $8.25-8.19(\mathrm{~m}, \mathrm{Ar}-\mathrm{H}, 2 \mathrm{H}), 7.75-7.70(\mathrm{~m}, \mathrm{Ar}-\mathrm{H}, 2 \mathrm{H}), 6.78(\mathrm{~d}, J=3.3 \mathrm{~Hz}$, Ar-H, 1H), $6.18-6.12(\mathrm{~m}, \mathrm{Ar}-\mathrm{H}, 1 \mathrm{H}), 2.41\left(\mathrm{~s}, \mathrm{CH}_{3}, 3 \mathrm{H}\right) .{ }^{13} \mathrm{C}$ NMR $(101$ $\left.\mathrm{MHz}, \mathrm{CDCl}_{3}\right) \delta 154.7,150.1,145.9,136.7,124.4,123.3,110.3,108.8,13.9$.

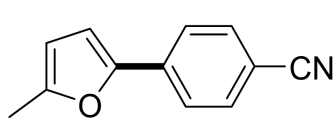

4-(5-methylfuran-2-yl)benzonitrile (3ab): ${ }^{1} \mathrm{H}$ NMR $\left(400 \mathrm{MHz}, \mathrm{CDCl}_{3}\right) \delta$ $7.69-7.66$ (m, Ar-H, 2H), $7.62(\mathrm{dt}, J=6.7,1.0 \mathrm{~Hz}, \mathrm{Ar}-\mathrm{H}, 2 \mathrm{H}), 6.70$ (d, $J=$ $3.3 \mathrm{~Hz}, \mathrm{Ar}-\mathrm{H}, 1 \mathrm{H}), 6.11(\mathrm{dq}, J=3.0,0.9 \mathrm{~Hz}, \mathrm{Ar}-\mathrm{H}, 1 \mathrm{H}), 2.39-2.38\left(\mathrm{~m}, \mathrm{CH}_{3}\right.$, 3H). ${ }^{13} \mathrm{C}$ NMR (101 MHz, $\left.\mathrm{CDCl}_{3}\right) \delta 154.0,150.3,134.9,132.5,123.3,119.1,109.4,109.3,108.5,13.8$.

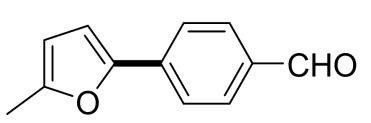

4-(5-methylfuran-2-yl)benzaldehyde (3ac): ${ }^{1} \mathrm{H}$ NMR $\left(400 \mathrm{MHz}, \mathrm{CDCl}_{3}\right)$ $\delta 9.89(\mathrm{~d}, J=1.8 \mathrm{~Hz}, \mathrm{Ar}-\mathrm{H}, 1 \mathrm{H}), 7.79$ (dd, $J=8.3,1.6 \mathrm{~Hz}, \mathrm{Ar}-\mathrm{H}, 2 \mathrm{H}), 7.68$ (dd, $J=8.2,1.5 \mathrm{~Hz}, \mathrm{Ar}-\mathrm{H}, 2 \mathrm{H}), 6.69-6.65$ (m, Ar-H, 1H), $6.07-6.04$ (m, $\mathrm{Ar}-\mathrm{H}, 1 \mathrm{H}), 2.33\left(\mathrm{~s}, \mathrm{CH}_{3}, 3 \mathrm{H}\right) .{ }^{13} \mathrm{C} \mathrm{NMR}\left(101 \mathrm{MHz}, \mathrm{CDCl}_{3}\right) \delta 191.6,154.0,151.0,136.4,134.4,130.4$, 123.3, 109.4, 108.5, 13.8 .

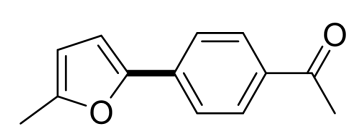

1-(4-(5-methylfuran-2-yl)phenyl)ethanone (3ad): ${ }^{1} \mathrm{H}$ NMR $(400 \mathrm{MHz}$, $\left.\mathrm{CDCl}_{3}\right) \delta 7.96(\mathrm{~d}, J=1.7 \mathrm{~Hz}, \mathrm{Ar}-\mathrm{H}, 1 \mathrm{H}), 7.94(\mathrm{~d}, J=1.8 \mathrm{~Hz}, \mathrm{Ar}-\mathrm{H}, 1 \mathrm{H})$, $7.70(\mathrm{~d}, J=1.8 \mathrm{~Hz}, \mathrm{Ar}-\mathrm{H}, 1 \mathrm{H}), 7.68$ (d, $J=1.7 \mathrm{~Hz}, \mathrm{Ar}-\mathrm{H}, 1 \mathrm{H}), 6.70$ (d, $J=$ $3.3 \mathrm{~Hz}, \mathrm{Ar}-\mathrm{H}, 1 \mathrm{H}), 6.11-6.10$ (m, Ar-H, 1H), $2.60\left(\mathrm{~s}, \mathrm{CH}_{3}, 3 \mathrm{H}\right), 2.39\left(\mathrm{~s}, \mathrm{COCH}_{3}, 3 \mathrm{H}\right) .{ }^{13} \mathrm{C}$ NMR $(101$ $\left.\mathrm{MHz}, \mathrm{CDCl}_{3}\right) \delta 197.4,153.5,151.1,135.1,134.9,128.9,122.9,108.6,108.3,26.5,13.8$.

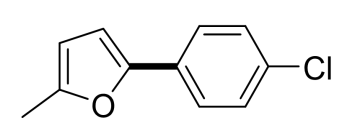

2-(4-chlorophenyl)-5-methylfuran (3ae): ${ }^{1} \mathrm{H}$ NMR (400 $\left.\mathrm{MHz}, \mathrm{CDCl}_{3}\right) \delta$ $7.55(\mathrm{~d}, J=8.7 \mathrm{~Hz}, \mathrm{Ar}-\mathrm{H}, 2 \mathrm{H}), 7.33-7.30(\mathrm{~m}, \mathrm{Ar}-\mathrm{H}, 2 \mathrm{H}), 6.52(\mathrm{~d}, J=3.2 \mathrm{~Hz}$, Ar-H, 1H), $6.07-6.03(\mathrm{~m}, \mathrm{Ar}-\mathrm{H}, 1 \mathrm{H}), 2.36\left(\mathrm{~d}, J=0.6 \mathrm{~Hz}, \mathrm{CH}_{3}, 3 \mathrm{H}\right) .{ }^{13} \mathrm{C}$ NMR (101 MHz, $\left.\mathrm{CDCl}_{3}\right) \delta 152.3,151.2,132.3,129.7,128.8,124.5,107.9,106.4,13.7$.

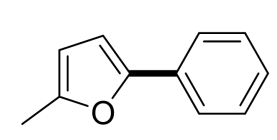

2-methyl-5-phenylfuran (3af): ${ }^{1} \mathrm{H}$ NMR $\left(400 \mathrm{MHz}, \mathrm{CDCl}_{3}\right) \delta 7.65-7.62(\mathrm{~m}$, Ar-H, 2H), $7.38-7.34$ (m, Ar-H, 2H), 7.22 (dt, J=9.0, 4.3 Hz, Ar-H, 1H), 6.54 (d, $J=3.2 \mathrm{~Hz}, \mathrm{Ar}-\mathrm{H}, 1 \mathrm{H}), 6.07-6.05(\mathrm{~m}, \mathrm{Ar}-\mathrm{H}, 1 \mathrm{H}), 2.38\left(\mathrm{~d}, J=0.5 \mathrm{~Hz}, \mathrm{CH}_{3}\right.$, 3H). ${ }^{13} \mathrm{C}$ NMR $\left(101 \mathrm{MHz}, \mathrm{CDCl}_{3}\right) \delta 152.3,151.9,131.2,128.6,126.7,123.3,107.7,105.8,13.7$.

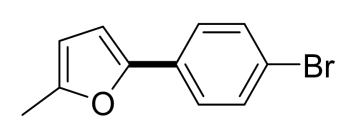

3-(4-bromophenyl)-5-methylfuran (3ag): ${ }^{1} \mathrm{H}$ NMR (400 $\left.\mathrm{MHz}, \mathrm{CDCl}_{3}\right) \delta$ 7.54 (s, Ar-H, 2H), 7.19 (s, Ar-H, 2H), 6.47 (d, J=3.2 Hz, Ar-H, 1H), $6.01-$ $5.97(\mathrm{~m}, \mathrm{Ar}-\mathrm{H}, 1 \mathrm{H}), 2.31\left(\mathrm{~d}, J=0.6 \mathrm{~Hz}, \mathrm{CH}_{3}, 3 \mathrm{H}\right) .{ }^{13} \mathrm{C} \mathrm{NMR}(101 \mathrm{MHz}$, $\left.\mathrm{CDCl}_{3}\right) \delta 152.2,152.0,129.4,129.3,123.5,123.1,107.8,105.8,13.7$. 


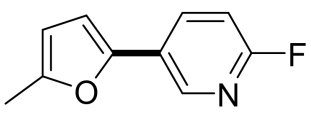

2-fluoro-5-(5-methylfuran-2-yl)pyridine (3ah): ${ }^{1} \mathrm{H}$ NMR $\left(400 \mathrm{MHz}, \mathrm{CDCl}_{3}\right)$ $\delta 8.46(\mathrm{dd}, J=1.5,0.9 \mathrm{~Hz}, \mathrm{Ar}-\mathrm{H}, 1 \mathrm{H}), 7.97$ (ddd, $J=8.5,7.6,2.5 \mathrm{~Hz}, \mathrm{Ar}-\mathrm{H}$, 1H), 6.92 (ddd, $J=8.6,3.0,0.6 \mathrm{~Hz}, \mathrm{Ar}-\mathrm{H}, 1 \mathrm{H}), 6.55$ (d, $J=3.2 \mathrm{~Hz}, \mathrm{Ar}-\mathrm{H}, 1 \mathrm{H}$ ), 6.07 (ddd, $J=3.0,1.9,0.9 \mathrm{~Hz}, \mathrm{Ar}-\mathrm{H}, 1 \mathrm{H}), 2.36$ (d, $\left.J=0.4 \mathrm{~Hz}, \mathrm{CH}_{3}, 3 \mathrm{H}\right) .{ }^{13} \mathrm{C} \mathrm{NMR}\left(101 \mathrm{MHz}, \mathrm{CDCl}_{3}\right)$ $\delta 163.4,161.0,153.0,148.3,142.5,142.4,135.8,135.8,125.5,125.5,109.6,109.2,107.9,107.0,107.0$, 13.6 .

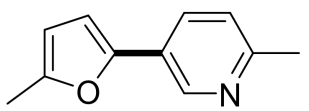

2-methyl-5-(5-methylfuran-2-yl)pyridine (3ai): ${ }^{1} \mathrm{H}$ NMR (400 $\left.\mathrm{MHz}, \mathrm{CDCl}_{3}\right)$ $\delta 8.75$ (d, $J=1.9 \mathrm{~Hz}, \mathrm{Ar}-\mathrm{H}, 1 \mathrm{H}), 7.77$ (dd, $J=8.1,2.3 \mathrm{~Hz}, \mathrm{Ar}-\mathrm{H}, 1 \mathrm{H}), 7.13$ (d, $J=8.1 \mathrm{~Hz}, \mathrm{Ar}-\mathrm{H}, 1 \mathrm{H}), 6.56$ (d, $J=3.2 \mathrm{~Hz}, \mathrm{Ar}-\mathrm{H}, 1 \mathrm{H}), 6.07-6.05$ (m, Ar-H, $1 \mathrm{H}), 2.55\left(\mathrm{~s}, \mathrm{CH}_{3}, 3 \mathrm{H}\right), 2.37\left(\mathrm{~s}, \mathrm{CH}_{3}, 3 \mathrm{H}\right) .{ }^{13} \mathrm{C} \mathrm{NMR}\left(101 \mathrm{MHz}, \mathrm{CDCl}_{3}\right) \delta 156.4,152.6,149.6,144.3$, $130.8,124.5,123.0,107.8,106.5,24.2,13.7$.

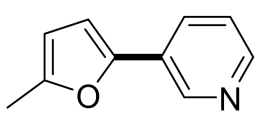

3-(5-methylfuran-2-yl)pyridine (3aj): ${ }^{1} \mathrm{H}$ NMR (400 $\left.\mathrm{MHz}, \mathrm{CDCl}_{3}\right) \delta 8.81$ (d, $J=$ $1.7 \mathrm{~Hz}, \mathrm{Ar}-\mathrm{H}, 1 \mathrm{H}), 8.37$ (dd, $J=4.8,1.5 \mathrm{~Hz}, \mathrm{Ar}-\mathrm{H}, 1 \mathrm{H}), 7.82-7.79$ (m, Ar-H, 1H), $7.22-7.18$ (m, Ar-H, 1H), 6.56 (d, J=3.2 Hz, Ar-H, 1H), $6.03-6.00$ (m, Ar-H, $1 \mathrm{H}), 2.31\left(\mathrm{~d}, J=0.5 \mathrm{~Hz}, \mathrm{CH}_{3}, 3 \mathrm{H}\right) .{ }^{13} \mathrm{C} \mathrm{NMR}\left(101 \mathrm{MHz}, \mathrm{CDCl}_{3}\right) \delta 153.1,149.3,147.6,145.0,130.2$, $127.2,123.5,108.0,107.4,13.7$.

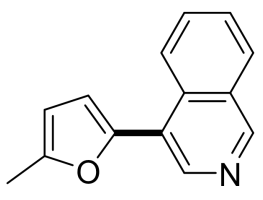

4-(5-methylfuran-2-yl)isoquinoline (3ak): ${ }^{1} \mathrm{H} \mathrm{NMR}\left(400 \mathrm{MHz}, \mathrm{CDCl}_{3}\right) \delta 9.16(\mathrm{~s}$, Ar-H, 1H), 8.75 (s, Ar-H, 1H), 8.44 (dd, $J=8.6,0.7 \mathrm{~Hz}, \mathrm{Ar}-\mathrm{H}, 1 \mathrm{H}), 8.00$ (d, $J=$ $8.2 \mathrm{~Hz}, \mathrm{Ar}-\mathrm{H}, 1 \mathrm{H}), 7.75$ (ddd, $J=8.4,6.9,1.3 \mathrm{~Hz}, \mathrm{Ar}-\mathrm{H}, 1 \mathrm{H}), 7.66-7.61$ (m, Ar-H, 1H), 6.70 (d, J=3.2 Hz, Ar-H, 1H), $6.22-6.16$ (m, Ar-H, 1H), 2.46 (d, $J=$ $\left.0.5 \mathrm{~Hz}, \mathrm{CH}_{3}, 3 \mathrm{H}\right) .{ }^{13} \mathrm{C} \mathrm{NMR}\left(101 \mathrm{MHz}, \mathrm{CDCl}_{3}\right) \delta 153.3,151.8,149.2,141.7,132.4,130.8,128.5$, $128.1,127.2,124.8,122.5,111.0,107.8,13.9$.

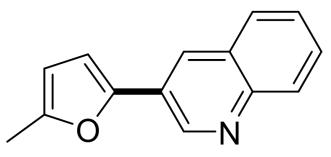

(5-methylfuran-2-yl)quinolone (3al): ${ }^{1} \mathrm{H}$ NMR $\left(400 \mathrm{MHz}, \mathrm{CDCl}_{3}\right) \delta 9.16(\mathrm{~d}$ $J=2.2 \mathrm{~Hz}, \mathrm{Ar}-\mathrm{H}, 1 \mathrm{H}), 8.29$ (s, Ar-H, 1H), 8.06 (d, $J=8.4 \mathrm{~Hz}, \mathrm{Ar}-\mathrm{H}, 1 \mathrm{H})$, $7.81(\mathrm{~d}, J=8.1 \mathrm{~Hz}, \mathrm{Ar}-\mathrm{H}, 1 \mathrm{H}), 7.64(\mathrm{t}, J=7.6 \mathrm{~Hz}, \mathrm{Ar}-\mathrm{H}, 1 \mathrm{H}), 7.52$ (dd, $J=$ 7.9, 7.1 Hz, Ar-H, 1H), 6.75 (d, J=3.2 Hz, Ar-H, 1H), 6.13 (dd, J=2.3, $1.0 \mathrm{~Hz}, \mathrm{Ar}-\mathrm{H}, 1 \mathrm{H}$ ), 2.42 (s, $\left.\mathrm{CH}_{3}, 3 \mathrm{H}\right) .{ }^{13} \mathrm{C}$ NMR $\left(101 \mathrm{MHz}, \mathrm{CDCl}_{3}\right) \delta 153.3,149.6,147.0,146.8,129.2,128.8,128.1,128.0,127.8$, 127.0, 124.3, 108.1, 107.7, 13.7.

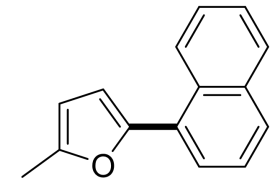

2-methyl-5-(naphthalen-1-yl)furan (3am): ${ }^{1} \mathrm{H}$ NMR $\left(400 \mathrm{MHz}, \mathrm{CDCl}_{3}\right) \delta 8.46$ (dd, $J=5.1,4.5 \mathrm{~Hz}, \mathrm{Ar}-\mathrm{H}, 1 \mathrm{H}), 7.89$ (dd, $J=7.1,2.4 \mathrm{~Hz}, \mathrm{Ar}-\mathrm{H}, 1 \mathrm{H}), 7.81$ (d, $J=$ $8.2 \mathrm{~Hz}, \mathrm{Ar}-\mathrm{H}, 1 \mathrm{H}), 7.73$ (dd, $J=7.2,1.1 \mathrm{~Hz}, \mathrm{Ar}-\mathrm{H}, 1 \mathrm{H}$ ), 7.53 (ddd, $J=6.9,4.5$, $1.3 \mathrm{~Hz}, \mathrm{Ar}-\mathrm{H}, 3 \mathrm{H}), 6.63$ (d, J=3.1 Hz, Ar-H, 1H), $6.20-6.18$ (m, Ar-H, 1H), 2.46 $\left(\mathrm{d}, J=0.5 \mathrm{~Hz}, \mathrm{CH}_{3}, 3 \mathrm{H}\right) .{ }^{13} \mathrm{C} \mathrm{NMR}\left(101 \mathrm{MHz}, \mathrm{CDCl}_{3}\right) \delta 152.2,151.6,134.0,130.2,128.9,128.5$, $128.0,126.3,125.8,125.6,125.6,125.3,110.2,107.5,13.8$.

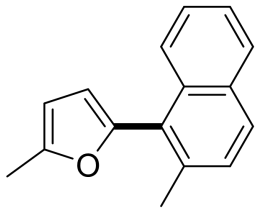

2-methyl-5-(2-methylnaphthalen-1-yl)furan (3an): ${ }^{1} \mathrm{H}$ NMR $\left(400 \mathrm{MHz}, \mathrm{CDCl}_{3}\right)$ $\delta 7.84-7.78$ (m, Ar-H, 3H), 7.42 (ddd, $J=13.8,7.9,5.4 \mathrm{~Hz}, \mathrm{Ar}-\mathrm{H}, 3 \mathrm{H}), 6.35$ (d, $J$ $=3.0 \mathrm{~Hz}, \mathrm{Ar}-\mathrm{H}, 1 \mathrm{H}), 6.20-6.19(\mathrm{~m}, \mathrm{Ar}-\mathrm{H}, 1 \mathrm{H}), 2.43\left(\mathrm{~s}, \mathrm{CH}_{3}, 3 \mathrm{H}\right), 2.42(\mathrm{~d}, J=0.7$ $\left.\mathrm{Hz}, \mathrm{CH}_{3}, 3 \mathrm{H}\right) .{ }^{13} \mathrm{C} \mathrm{NMR}\left(101 \mathrm{MHz}, \mathrm{CDCl}_{3}\right) \delta 151.8,149.3,136.3,133.4,132.0$, 


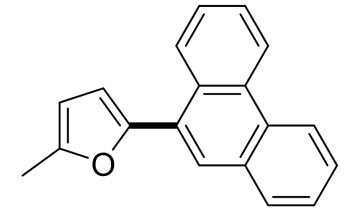

2-methyl-5-(phenanthren-9-yl)furan (3ao): ${ }^{1} \mathrm{H}$ NMR $\left(400 \mathrm{MHz}, \mathrm{CDCl}_{3}\right) \delta$ $8.78(\mathrm{dd}, J=8.4,1.1 \mathrm{~Hz}, \mathrm{Ar}-\mathrm{H}, 1 \mathrm{H}), 8.71-8.68$ (m, Ar-H, 1H), $8.54-8.51$ (m, Ar-H, 1H), $8.02(\mathrm{~s}, \mathrm{Ar}-\mathrm{H}, 1 \mathrm{H}), 7.95-7.92(\mathrm{~m}, \mathrm{Ar}-\mathrm{H}, 1 \mathrm{H}), 7.76-7.58(\mathrm{~m}$, Ar-H, 5H), 6.71 (d, J=3.1 Hz, Ar-H, 1H), 6.26-6.22 (m, Ar-H, 1H), 2.51 (d, $\left.J=0.7 \mathrm{~Hz}, \mathrm{CH}_{3}, 3 \mathrm{H}\right) .{ }^{13} \mathrm{C} \mathrm{NMR}\left(101 \mathrm{MHz}, \mathrm{CDCl}_{3}\right) \delta 152.3,151.5,131.4,130.7,130.0,129.5,128.8$, $127.5,126.8,126.8,126.7,126.6,126.5,126.3,123.0,122.5,110.6,107.4,13.8$.

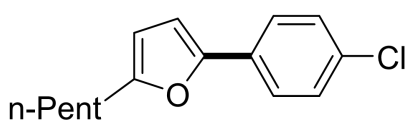

2-(4-chlorophenyl)-5-pentylfuran (3be): ${ }^{1} \mathrm{H} \mathrm{NMR}\left(400 \mathrm{MHz}, \mathrm{CDCl}_{3}\right)$ $\delta 7.61-7.51$ (m, Ar-H, 2H), $7.34-7.30$ (m, Ar-H, 2H), 6.54 (d, J=3.2 $\mathrm{Hz}, \mathrm{Ar}-\mathrm{H}, 1 \mathrm{H}), 6.06$ (dt, $J=3.2,0.8 \mathrm{~Hz}, \mathrm{Ar}-\mathrm{H}, 1 \mathrm{H}), 2.67$ (t, $J=7.6 \mathrm{~Hz}$, $\left.\mathrm{CH}_{2}, 2 \mathrm{H}\right), 1.70\left(\mathrm{~s}, \mathrm{CH}_{2}, 2 \mathrm{H}\right), 1.40-1.35\left(\mathrm{~m}, \mathrm{CH}_{2}, 4 \mathrm{H}\right), 0.92(\mathrm{ddd}, J=7.1,5.2,1.9 \mathrm{~Hz}, 3 \mathrm{H}) .{ }^{13} \mathrm{C}$ NMR $\left(101 \mathrm{MHz}, \mathrm{CDCl}_{3}\right) \delta 156.8,151.0,132.2,129.7,128.7,124.5,107.0,106.1,31.4,28.1,27.7,22.4$, 14.0 .

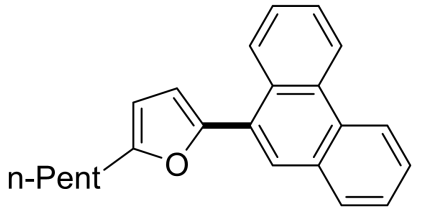

2-pentyl-5-(phenanthren-9-yl)furan (3bo): ${ }^{1} \mathrm{H}$ NMR (400 $\mathrm{MHz}$, $\left.\mathrm{CDCl}_{3}\right) \delta 8.79-8.76(\mathrm{~m}, \mathrm{Ar}-\mathrm{H}, 1 \mathrm{H}), 8.69(\mathrm{dd}, J=8.1,0.5 \mathrm{~Hz}, \mathrm{Ar}-\mathrm{H}$, $1 \mathrm{H}), 8.52-8.48(\mathrm{~m}, \mathrm{Ar}-\mathrm{H}, 1 \mathrm{H}), 8.00(\mathrm{~s}, \mathrm{Ar}-\mathrm{H}, 1 \mathrm{H}), 7.95-7.91$ (m, Ar-H, 1H), $7.73-7.59$ (m, Ar-H, 4H), 6.71 (d, $J=3.2 \mathrm{~Hz}, \mathrm{Ar}-\mathrm{H}, 1 \mathrm{H})$, $6.23(\mathrm{dt}, J=3.1,0.8 \mathrm{~Hz}, \mathrm{Ar}-\mathrm{H}, 1 \mathrm{H}), 2.81$ (t, $\left.J=7.6 \mathrm{~Hz}, \mathrm{CH}_{2}, 2 \mathrm{H}\right), 1.80$ (d, $\left.J=7.6 \mathrm{~Hz}, \mathrm{CH}_{2}, 2 \mathrm{H}\right), 1.49-$ $1.41\left(\mathrm{~m}, \mathrm{CH}_{2}, 4 \mathrm{H}\right), 0.98\left(\mathrm{t}, J=7.1 \mathrm{~Hz}, \mathrm{CH}_{3}, 3 \mathrm{H}\right) .{ }^{13} \mathrm{C} \mathrm{NMR}\left(101 \mathrm{MHz}, \mathrm{CDCl}_{3}\right) \delta 156.8,151.4,131.5$, $130.8,130.0,129.6,128.8,127.7,126.8,126.8,126.7,126.6,126.5,126.4,123.0,122.5,110.3,106.6$, $31.5,28.2,27.9,22.5,14.1$.<smiles>P[Pb]c1ccc(-c2ccc(Cl)nc2)o1</smiles>

2-chloro-5-(5-pentylfuran-2-yl)pyridine (3bp): ${ }^{1} \mathrm{H}$ NMR (400 MHz, $\left.\mathrm{CDCl}_{3}\right) \delta 8.55(\mathrm{~s}, \mathrm{Ar}-\mathrm{H}, 1 \mathrm{H}), 7.82-7.67(\mathrm{~m}, \mathrm{Ar}-\mathrm{H}, 1 \mathrm{H}), 7.22(\mathrm{~d}, J=6.7$ $\mathrm{Hz}, \mathrm{Ar}-\mathrm{H}, 1 \mathrm{H}), 6.55$ (s, Ar-H, 1H), 6.02 (s, Ar-H, 1H), 2.60 (s, $\mathrm{CH}_{2}$, $2 \mathrm{H}), 1.61\left(\mathrm{~s}, \mathrm{CH}_{2}, 2 \mathrm{H}\right), 1.28\left(\mathrm{~s}, \mathrm{CH}_{2}, 4 \mathrm{H}\right), 0.83\left(\mathrm{~s}, \mathrm{CH}_{3}, 3 \mathrm{H}\right) .{ }^{13} \mathrm{C} \mathrm{NMR}\left(101 \mathrm{MHz}, \mathrm{CDCl}_{3}\right) \delta 158.0$, $148.8,147.9,144.5,133.0,126.2,124.0,107.8,107.2,31.3,28.0,27.6,22.3,13.9$.<smiles>[PH]c1ccc(-c2cncnc2)o1</smiles>

5-(5-pentylfuran-2-yl)pyrimidine (3bq): ${ }^{1} \mathrm{H}$ NMR $\left(400 \mathrm{MHz}, \mathrm{CDCl}_{3}\right) \delta$ 9.03 (s, Ar-H, 1H), 8.93 (s, Ar-H, 2H), 6.71 (d, $J=3.3 \mathrm{~Hz}, \mathrm{Ar}-\mathrm{H}, 1 \mathrm{H}), 6.12$ $(\mathrm{dt}, J=3.3,0.8 \mathrm{~Hz}, \mathrm{Ar}-\mathrm{H}, 1 \mathrm{H}), 2.68\left(\mathrm{t}, J=7.6 \mathrm{~Hz}, \mathrm{CH}_{2}, 2 \mathrm{H}\right), 1.68\left(\mathrm{~s}, \mathrm{CH}_{2}\right.$, $2 \mathrm{H}), 1.38-1.33\left(\mathrm{~m}, \mathrm{CH}_{2}, 4 \mathrm{H}\right), 0.92-0.88\left(\mathrm{~m}, \mathrm{CH}_{3}, 3 \mathrm{H}\right) .{ }^{13} \mathrm{C} \mathrm{NMR}\left(101 \mathrm{MHz}, \mathrm{CDCl}_{3}\right) \delta 158.8,156.3$, $151.2,145.9,125.3,108.8,107.4,31.3,28.0,27.6,22.3,13.9$.

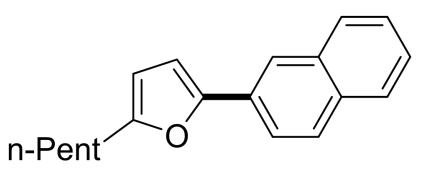

(naphthalen-2-yl)-5-pentylfuran (3br): ${ }^{1} \mathrm{H}$ NMR $\left(400 \mathrm{MHz}, \mathrm{CDCl}_{3}\right)$ $\delta 8.09$ (s, Ar-H, 1H), $7.87-7.78$ (m, Ar-H, 3H), 7.74 (dd, $J=8.6,1.3$ $\mathrm{Hz}, \mathrm{Ar}-\mathrm{H}, 1 \mathrm{H}), 7.49-7.40$ (m, Ar-H, 2H), 6.67 (d, $J=3.2 \mathrm{~Hz}, \mathrm{Ar}-\mathrm{H}$, $1 \mathrm{H}), 6.11(\mathrm{~d}, J=3.2 \mathrm{~Hz}, \mathrm{Ar}-\mathrm{H}, 1 \mathrm{H}), 2.73\left(\mathrm{t}, J=7.6 \mathrm{~Hz}, \mathrm{CH}_{2}, 2 \mathrm{H}\right), 1.78$ $-1.71\left(\mathrm{~m}, \mathrm{CH}_{2}, 2 \mathrm{H}\right), 1.45-1.36\left(\mathrm{~m}, \mathrm{CH}_{2}, 4 \mathrm{H}\right), 0.94\left(\mathrm{t}, J=6.6 \mathrm{~Hz}, \mathrm{CH}_{3}, 3 \mathrm{H}\right) .{ }^{13} \mathrm{C} \mathrm{NMR}(101 \mathrm{MHz}$, $\left.\mathrm{CDCl}_{3}\right) \delta 156.9,152.1,133.6,132.4,128.6,128.2,128.0,127.7,126.3,125.5,122.2,121.2,107.0$, $106.4,31.4,28.2,27.8,22.4,14.0$. 
<smiles>O=Cc1ccc(-c2ccc([N+](=O)[O-])cc2)o1</smiles>

4-(4-nitrophenyl)furan-2-carbaldehyde (3ca): ${ }^{1} \mathrm{H}$ NMR (400 MHz, $\left.\mathrm{CDCl}_{3}\right) \delta 9.73(\mathrm{~s}, \mathrm{CHO}, 1 \mathrm{H}), 8.30$ (s, Ar-H, 2H), 7.99 (s, Ar-H, 2H), 7.37 (d, $J=3.8 \mathrm{~Hz}, \mathrm{Ar}-\mathrm{H}, 1 \mathrm{H}), 7.04$ (d, $J=3.8 \mathrm{~Hz}, \mathrm{Ar}-\mathrm{H}, 1 \mathrm{H}) .{ }^{13} \mathrm{C}$ NMR (101 MHz, $\left.\mathrm{CDCl}_{3}\right) \delta$ 177.6, 156.3, 153.0, 147.9, 134.5, 125.8, 124.4, 122.8, 110.6.

(quinolin-3-yl)furan-2-carbaldehyde (3cl): ${ }^{1} \mathrm{H}$ NMR $\left(400 \mathrm{MHz}, \mathrm{CDCl}_{3}\right)$ $\delta 9.73(\mathrm{~s}, \mathrm{CHO}, 1 \mathrm{H}), 9.28(\mathrm{~d}, J=2.2 \mathrm{~Hz}, \mathrm{Ar}-\mathrm{H}, 1 \mathrm{H}), 8.64(\mathrm{~d}, J=2.1 \mathrm{~Hz}$, Ar-H, $1 \mathrm{H}), 8.13(\mathrm{~d}, J=8.2 \mathrm{~Hz}, \mathrm{Ar}-\mathrm{H}, 1 \mathrm{H}), 7.91(\mathrm{dd}, J=8.2,1.2 \mathrm{~Hz}$, Ar-H, 1H), 7.77 (ddd, $J=8.4,6.9,1.4 \mathrm{~Hz}, \mathrm{Ar}-\mathrm{H}, 1 \mathrm{H}), 7.62$ (ddd, $J=8.1$, 7.0, 1.1 Hz, Ar-H, 1H), 7.40 (d, $J=3.7 \mathrm{~Hz}, \mathrm{Ar}-\mathrm{H}, 1 \mathrm{H}), 7.07$ (d, $J=3.7 \mathrm{~Hz}, \mathrm{Ar}-\mathrm{H}, 1 \mathrm{H}) .{ }^{13} \mathrm{C}$ NMR $(101$ $\left.\mathrm{MHz}, \mathrm{CDCl}_{3}\right) \delta 179.8,150.3,147.5,140.5,135.8,133.6,130.33,129.4,128.0,127.5,127.3,124.4$, 124.3, 111.7, 34.4.<smiles>COc1ccc(-c2ccc(C=O)o2)cn1</smiles>

(6-methoxypyridin-3-yl)furan-2-carbaldehyde (3cs): ${ }^{1} \mathrm{H}$ NMR (400 $\left.\mathrm{MHz}, \mathrm{CDCl}_{3}\right) \delta 9.64(\mathrm{~s}, \mathrm{CHO}, 1 \mathrm{H}), 8.63(\mathrm{dd}, J=2.4,0.6 \mathrm{~Hz}, \mathrm{Ar}-\mathrm{H}, 1 \mathrm{H})$, 8.00 (dd, $J=8.7,2.5 \mathrm{~Hz}, \mathrm{Ar}-\mathrm{H}, 1 \mathrm{H}), 7.32$ (d, $J=3.7 \mathrm{~Hz}, \mathrm{Ar}-\mathrm{H}, 1 \mathrm{H}), 6.83$ (dd, $J=8.7,0.7 \mathrm{~Hz}, \mathrm{Ar}-\mathrm{H}, 1 \mathrm{H}), 6.77$ (d, $J=3.7 \mathrm{~Hz}, \mathrm{Ar}-\mathrm{H}, 1 \mathrm{H}), 3.99$ (s, $\left.\mathrm{OCH}_{3}, 3 \mathrm{H}\right) .{ }^{13} \mathrm{C}$ NMR $(101$ $\left.\mathrm{MHz}, \mathrm{CDCl}_{3}\right) \delta 177.0,164.8,157.3,152.0,144.6,135.4,119.0,111.3,106.9,53.9,29.7$.

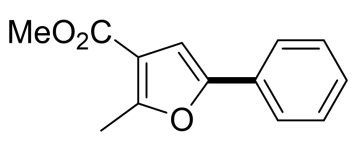

methyl 2-methyl-5-phenylfuran-3-carboxylate (3df): ${ }^{1} \mathrm{H}$ NMR $(400 \mathrm{MHz}$, $\left.\mathrm{CDCl}_{3}\right) \delta 7.64(\mathrm{~d}, J=1.3 \mathrm{~Hz}, \mathrm{Ar}-\mathrm{H}, 1 \mathrm{H}), 7.62(\mathrm{~d}, J=1.1 \mathrm{~Hz}, \mathrm{Ar}-\mathrm{H}, 1 \mathrm{H})$, 7.37 (dd, $J=8.2,7.1 \mathrm{~Hz}, \mathrm{Ar}-\mathrm{H}, 2 \mathrm{H}), 7.29-7.24$ (m, Ar-H, 1H), 6.87 (s, $\mathrm{Ar}-\mathrm{H}, 1 \mathrm{H}), 3.84\left(\mathrm{~s}, \mathrm{COOCH}_{3}, 3 \mathrm{H}\right), 2.64\left(\mathrm{~s}, \mathrm{CH}_{3}, 3 \mathrm{H}\right) .{ }^{13} \mathrm{C} \mathrm{NMR}\left(101 \mathrm{MHz}, \mathrm{CDCl}_{3}\right) \delta$ 164.4, 158.7, $151.7,130.0,128.7,127.6,123.6,115.1,105.4,51.4,13.8$.

$\mathrm{MeO}_{2} \mathrm{C}$

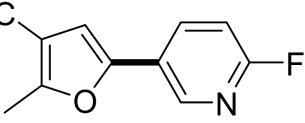

methyl 5-(6-fluoropyridin-3-yl)-2-methylfuran-3-carboxylate (3dh):

${ }^{1} \mathrm{H}$ NMR $\left(400 \mathrm{MHz}, \mathrm{CDCl}_{3}\right) \delta 8.48(\mathrm{~s}, \mathrm{Ar}-\mathrm{H}, 1 \mathrm{H}), 8.01-7.95(\mathrm{~m}, \mathrm{Ar}-\mathrm{H}$, $1 \mathrm{H}), 6.95$ (dd, $J=8.5,2.9 \mathrm{~Hz}, \mathrm{Ar}-\mathrm{H}, 1 \mathrm{H}), 6.90$ (s, Ar-H, 1H), 3.85 (s, $\left.\mathrm{COOCH}_{3}, 3 \mathrm{H}\right), 2.64\left(\mathrm{~s}, \mathrm{CH}_{3}, 3 \mathrm{H}\right) .{ }^{13} \mathrm{C} \mathrm{NMR}\left(101 \mathrm{MHz}, \mathrm{CDCl}_{3}\right) \delta 164.0,163.9,161.5,159.6,147.9$, 143.0, 142.9, 136.3, 136.2, 124.4, 124.4, 115.3, 109.9, 109.5, 106.7, 51.5, 13.8 .

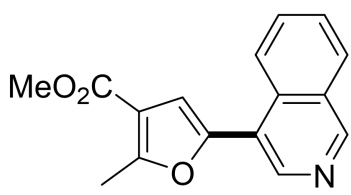

methyl 5-(isoquinolin-4-yl)-2-methylfuran-3-carboxylate (3dk): $\quad{ }^{1} \mathrm{H}$ NMR $\left(400 \mathrm{MHz}, \mathrm{CDCl}_{3}\right) \delta 9.17(\mathrm{~d}, J=2.2 \mathrm{~Hz}, \mathrm{Ar}-\mathrm{H}, 1 \mathrm{H}), 8.35(\mathrm{~d}, J=2.1$ $\mathrm{Hz}, \mathrm{Ar}-\mathrm{H}, 1 \mathrm{H}), 8.09$ (d, $J=8.4 \mathrm{~Hz}, \mathrm{Ar}-\mathrm{H}, 1 \mathrm{H}), 7.85$ (dd, $J=8.1,1.2 \mathrm{~Hz}$, Ar-H, 1H), 7.70 (ddd, $J=8.4,6.9,1.4 \mathrm{~Hz}, \mathrm{Ar}-\mathrm{H}, 1 \mathrm{H}), 7.57$ (ddd, $J=8.1$, 6.9, $1.2 \mathrm{~Hz}, \mathrm{Ar}-\mathrm{H}, 1 \mathrm{H}), 7.11(\mathrm{~s}, \mathrm{Ar}-\mathrm{H}, 1 \mathrm{H}), 3.88\left(\mathrm{~s}, \mathrm{COOCH}_{3}, 3 \mathrm{H}\right), 2.72\left(\mathrm{~s}, \mathrm{CH}_{3}, 3 \mathrm{H}\right) .{ }^{13} \mathrm{C} \mathrm{NMR}(101$ $\left.\mathrm{MHz}, \mathrm{CDCl}_{3}\right) \delta 164.1,159.9,149.2,147.2,146.8,129.5,129.4,129.1,128.0,127.7,127.3,123.3$, $115.5,107.2,51.5,14.0$.<smiles>CC(=O)c1cc(-c2c(C)ccc3ccccc23)oc1C</smiles>

methyl 2-methyl-5-(2-methylnaphthalen-1-yl)furan-3-carboxylate (3dn): ${ }^{1} \mathrm{H}$ NMR (400 MHz, $\left.\mathrm{CDCl}_{3}\right) \delta 7.85-7.81(\mathrm{~m}, \mathrm{Ar}-\mathrm{H}, 2 \mathrm{H}), 7.77-$ 7.73 (m, Ar-H, 1H), 7.47 - 7.42 (m, Ar-H, 2H), 7.40 (d, J=8.4 Hz, Ar-H, 1H), $6.75(\mathrm{~s}, \mathrm{Ar}-\mathrm{H}, 1 \mathrm{H}), 3.90\left(\mathrm{~s}, \mathrm{COOCH}_{3}, 3 \mathrm{H}\right), 2.71\left(\mathrm{~s}, \mathrm{CH}_{3}, 3 \mathrm{H}\right), 2.43(\mathrm{~s}$, 
$\left.\mathrm{CH}_{3}, 3 \mathrm{H}\right) .{ }^{13} \mathrm{C} \mathrm{NMR}\left(101 \mathrm{MHz}, \mathrm{CDCl}_{3}\right) \delta 164.7,159.1,149.1,136.7,133.2,131.9,129.2,128.4,127.9$, $126.6,126.3,125.3,125.1,114.2,111.2,51.3,20.7,13.9$.

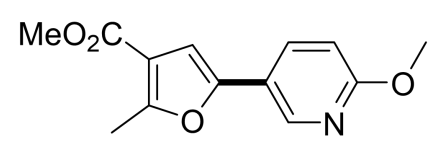

5-(6-methoxypyridin-3-yl)furan-2-carbaldehyde (3ds): ${ }^{1} \mathrm{H} \quad \mathrm{NMR}$ $\left(400 \mathrm{MHz}, \mathrm{CDCl}_{3}\right) \delta 8.41(\mathrm{~d}, J=2.0 \mathrm{~Hz}, \mathrm{Ar}-\mathrm{H}, 1 \mathrm{H}), 7.76(\mathrm{dd}, J=8.7$, $2.5 \mathrm{~Hz}, \mathrm{Ar}-\mathrm{H}, 1 \mathrm{H}), 6.76-6.72(\mathrm{~m}, \mathrm{Ar}-\mathrm{H}, 2 \mathrm{H}), 3.93\left(\mathrm{~s}, \mathrm{COOCH}_{3}, 3 \mathrm{H}\right)$, $3.83\left(\mathrm{~s}, \mathrm{OCH}_{3}, 3 \mathrm{H}\right), 2.61\left(\mathrm{~s}, \mathrm{CH}_{3}, 3 \mathrm{H}\right) .{ }^{13} \mathrm{C} \mathrm{NMR}\left(101 \mathrm{MHz}, \mathrm{CDCl}_{3}\right) \delta 164.3,163.5,158.7,149.3,142.4$, $134.2,119.9,115.0,110.9,104.7,53.5,51.4,13.8$.

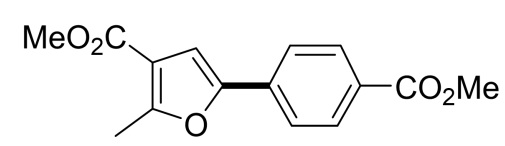

methyl

5-(4-(methoxycarbonyl)phenyl)-2-methylfuran-3-carboxylate (3dt): ${ }^{1} \mathrm{H}$ NMR $\left(400 \mathrm{MHz}, \mathrm{CDCl}_{3}\right) \delta 8.06(\mathrm{~d}, J=1.8 \mathrm{~Hz}, \mathrm{Ar}-\mathrm{H}$, 1H), 8.04 (d, $J=1.8 \mathrm{~Hz}, \mathrm{Ar}-\mathrm{H}, 1 \mathrm{H}), 7.69$ (d, $J=1.8 \mathrm{~Hz}, \mathrm{Ar}-\mathrm{H}, 1 \mathrm{H}), 7.68$ (d, $J=1.7 \mathrm{~Hz}, \mathrm{Ar}-\mathrm{H}, 1 \mathrm{H})$, $7.01(\mathrm{~s}, \mathrm{Ar}-\mathrm{H}, 1 \mathrm{H}), 3.92\left(\mathrm{~s}, \mathrm{COOCH}_{3}, 3 \mathrm{H}\right), 3.86\left(\mathrm{~s}, \mathrm{COOCH}_{3}, 3 \mathrm{H}\right), 2.67\left(\mathrm{~s}, \mathrm{CH}_{3}, 3 \mathrm{H}\right) .{ }^{13} \mathrm{C} \mathrm{NMR}(101$ $\left.\mathrm{MHz}_{2} \mathrm{CDCl}_{3}\right) \delta 166.7,164.2,159.8,150.7,133.9,130.1,128.8,123.2,115.5,107.7,52.1,51.5,13.9$.

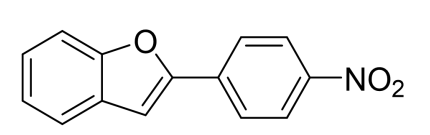

2-(4-nitrophenyl)benzofuran (3ea): ${ }^{1} \mathrm{H}$ NMR (400 $\left.\mathrm{MHz}, \mathrm{CDCl} 3\right) \delta$ $8.33-8.28$ (m, Ar-H, 2H), $8.02-7.97$ (m, Ar-H, 2H), 7.64 (ddd, J = 7.7, 1.2, 0.7 Hz, Ar-H, 1H), 7.56 (dd, J = 8.3, 0.8 Hz, Ar-H, 1H), 7.37 (ddd, J $=8.4,7.3,1.3 \mathrm{~Hz}, \mathrm{Ar}-\mathrm{H}, 1 \mathrm{H}), 7.31-7.26(\mathrm{~m}, \mathrm{Ar}-\mathrm{H}, 1 \mathrm{H}), 7.24$ (d, J = 0.9 Hz, Ar-H, 1H). ${ }^{13} \mathrm{C}$ NMR $(101$ $\mathrm{MHz}, \mathrm{CDCl} 3) \delta 155.4,153.2,147.2,136.3,128.6,125.8,125.2,124.3,123.5,121.6,111.5,105.1$.

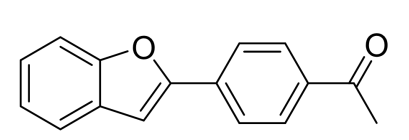

1-(4-(benzofuran-2-yl)phenyl)ethanone (3ed): ${ }^{1} \mathrm{H}$ NMR $(400 \mathrm{MHz}$, $\left.\mathrm{CDCl}_{3}\right) \delta 8.06-8.02(\mathrm{~m}, \mathrm{Ar}-\mathrm{H}, 2 \mathrm{H}), 7.98-7.93(\mathrm{~m}, \mathrm{Ar}-\mathrm{H}, 2 \mathrm{H}), 7.62(\mathrm{ddd}$, $J=7.7,1.3,0.6 \mathrm{~Hz}, \mathrm{Ar}-\mathrm{H}, 1 \mathrm{H}), 7.55$ (dd, $J=8.2,0.8 \mathrm{~Hz}, \mathrm{Ar}-\mathrm{H}, 1 \mathrm{H}), 7.34$ (ddd, $J=8.3,7.3,1.4 \mathrm{~Hz}$, Ar-H, 1H), 7.28-7.24 (dd, $J=1.0 \mathrm{~Hz}, \mathrm{Ar}-\mathrm{H}, 2 \mathrm{H}$ ), 7.18 (d, $J=0.9 \mathrm{~Hz}, \mathrm{Ar}-\mathrm{H}$, $1 \mathrm{H}), 2.64\left(\mathrm{~s}, \mathrm{CH}_{3}, 3 \mathrm{H}\right) \cdot{ }^{13} \mathrm{C} \mathrm{NMR}\left(101 \mathrm{MHz}, \mathrm{CDCl}_{3}\right) \delta 197.4,155.2,154.5,136.5,134.6,128.9,128.9$, 125.2, 124.8, 123.3, 121.3, 111.4, 103.7, 26.6 .

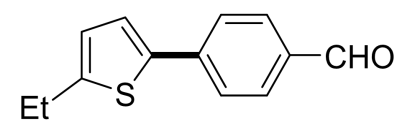

4-(5-ethylthiophen-2-yl)benzaldehyde (5ac): ${ }^{1} \mathrm{H}$ NMR $(400 \mathrm{MHz}$, $\left.\mathrm{CDCl}_{3}\right) \delta 9.97(\mathrm{~s}, \mathrm{CHO}, 1 \mathrm{H}), 7.85(\mathrm{~d}, J=8.2 \mathrm{~Hz}, \mathrm{Ar}-\mathrm{H}, 2 \mathrm{H}), 7.70(\mathrm{~d}, J=$ $8.2 \mathrm{~Hz}, \mathrm{Ar}-\mathrm{H}, 2 \mathrm{H}), 7.29$ (d, $J=3.5 \mathrm{~Hz}, \mathrm{Ar}-\mathrm{H}, 1 \mathrm{H}), 6.82$ (d, $J=3.4 \mathrm{~Hz}$, Ar-H, 1H), 2.88 (q, $\left.J=7.5 \mathrm{~Hz}, \mathrm{CH}_{2}, 2 \mathrm{H}\right), 1.35$ (t, $\left.J=7.5 \mathrm{~Hz}, \mathrm{CH}_{3}, 3 \mathrm{H}\right) .{ }^{13} \mathrm{C} \mathrm{NMR}\left(101 \mathrm{MHz}, \mathrm{CDCl}_{3}\right) \delta$ $191.5,161.7,149.8,140.5,139.8,134.6,130.4,125.4,124.9,23.7,15.8$.

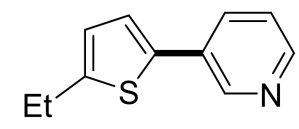

(5-ethylthiophen-2-yl)pyridine (5aj): ${ }^{1} \mathrm{H}$ NMR (400 MHz, $\left.\mathrm{CDCl}_{3}\right) \delta 8.73$ (s, Ar-H, 1H), 8.40 - 8.29 (m, Ar-H, 1H), $7.71-7.60$ (m, Ar-H, 1H), 7.15 - 7.00 (m, Ar-H, 2H), $6.71-6.60$ (m, Ar-H, 1H), 2.73 (dd, $\left.J=9.6,3.1 \mathrm{~Hz}, \mathrm{CH}_{2}, 2 \mathrm{H}\right), 1.21$ $\left(\mathrm{ddd}, J=7.5,6.8,4.5 \mathrm{~Hz}, \mathrm{CH}_{3}, 3 \mathrm{H}\right) .{ }^{13} \mathrm{C} \mathrm{NMR}\left(101 \mathrm{MHz}, \mathrm{CDCl}_{3}\right) \delta 148.1,147.3,146.0,137.0,131.9$, $130.3,124.2,123.5,123.1,23.1,15.4$.

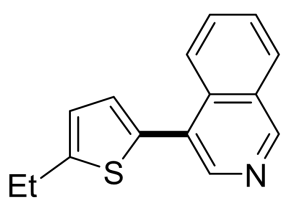

4-(5-ethylthiophen-2-yl)isoquinoline (5ak): ${ }^{1} \mathrm{H}$ NMR $\left(400 \mathrm{MHz}, \mathrm{CDCl}_{3}\right) \delta 9.15$ (s, Ar-H, 1H), 8.57 (s, Ar-H, 1H), 8.24 (d, $J=8.5 \mathrm{~Hz}, \mathrm{Ar}-\mathrm{H}, 1 \mathrm{H}), 7.92$ (d, $J=8.1$ $\mathrm{Hz}, \mathrm{Ar}-\mathrm{H}, 1 \mathrm{H}), 7.69-7.59$ (m, Ar-H, 1H), 7.54 (t, J= $7.1 \mathrm{~Hz}, \mathrm{Ar}-\mathrm{H}, 1 \mathrm{H}), 7.06$ (d, 
$J=3.4 \mathrm{~Hz}, \mathrm{Ar}-\mathrm{H}, 1 \mathrm{H}), 6.85(\mathrm{dd}, J=2.4,0.9 \mathrm{~Hz}, \mathrm{Ar}-\mathrm{H}, 1 \mathrm{H}), 2.88\left(\mathrm{q}, J=7.5 \mathrm{~Hz}, \mathrm{CH}_{2}, 2 \mathrm{H}\right), 1.35$ (t, $J=$ $\left.7.5 \mathrm{~Hz}, \mathrm{CH}_{3}, 3 \mathrm{H}\right) .{ }^{13} \mathrm{C} \mathrm{NMR}\left(101 \mathrm{MHz}, \mathrm{CDCl}_{3}\right) \delta 151.7,148.7,143.0,134.7,133.8,130.5,128.1,127.6$, 127.6, 127.0, 126.4, 124.4, 123.8, 23.3, 15.7.

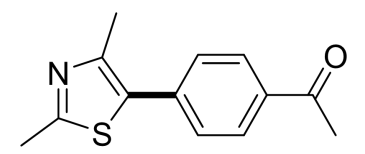

(4-(2,4-dimethylthiazol-5-yl)phenyl)ethanone (5bd): ${ }^{1} \mathrm{H}$ NMR $(400 \mathrm{MHz}$, $\left.\mathrm{CDCl}_{3}\right) \delta 8.00-7.97$ (m, Ar-H, 2H), $7.51-7.48$ (m, Ar-H, 2H), 2.69 (s, $\mathrm{CH}_{3}$, $3 \mathrm{H}), 2.61\left(\mathrm{~s}, \mathrm{CH}_{3}, 3 \mathrm{H}\right), 2.48\left(\mathrm{~s}, \mathrm{CH}_{3}, 3 \mathrm{H}\right) \cdot{ }^{13} \mathrm{C} \mathrm{NMR}\left(101 \mathrm{MHz}, \mathrm{CDCl}_{3}\right) \delta$ 197.3, 164.3, 148.3, 137.2, 135.8, 130.3, 129.0, 128.7, 26.6, 19.1, 16.3.

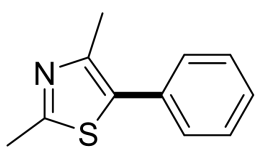

$127.5,19.1,16.0$

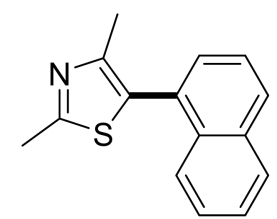

2,4-dimethyl-5-(naphthalen-1-yl)thiazole (5bm): ${ }^{1} \mathrm{H}$ NMR $\left(400 \mathrm{MHz}, \mathrm{CDCl}_{3}\right) \delta$ 7.93 - 7.89 (m, Ar-H, 2H), 7.80 - 7.76 (m, Ar-H, 1H), 7.53 - 7.47 (m, Ar-H, 4H), $2.77\left(\mathrm{~s}, \mathrm{CH}_{3}, 3 \mathrm{H}\right), 2.22\left(\mathrm{~s}, \mathrm{CH}_{3}, 3 \mathrm{H}\right) .{ }^{13} \mathrm{C} \mathrm{NMR}\left(101 \mathrm{MHz}, \mathrm{CDCl}_{3}\right) \delta 164.4,149.3$, $133.6,132.4$, 129.2, 129.2, 128.9, 128.3, 128.3, 126.5, 126.1, 125.6, 125.1, 19.1,

15.7 .

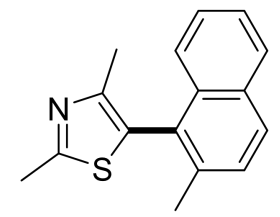

2,4-dimethyl-5-(2-methylnaphthalen-1-yl)thiazole (5bn): ${ }^{1} \mathrm{H}$ NMR (400 MHz, $\left.\mathrm{CDCl}_{3}\right) \delta 7.86-7.80(\mathrm{~m}, \mathrm{Ar}-\mathrm{H}, 2 \mathrm{H}), 7.53(\mathrm{dd}, J=8.0,1.7 \mathrm{~Hz}, \mathrm{Ar}-\mathrm{H}, 1 \mathrm{H}), 7.44-$ 7.40 (m, Ar-H, 3H), 2.77 (s, $\left.\mathrm{CH}_{3}, 3 \mathrm{H}\right), 2.31\left(\mathrm{~s}, \mathrm{CH}_{3}, 3 \mathrm{H}\right), 2.08\left(\mathrm{~s}, \mathrm{CH}_{3}, 3 \mathrm{H}\right) .{ }^{13} \mathrm{C}$ NMR $\left(101 \mathrm{MHz}, \mathrm{CDCl}_{3}\right) \delta 165.1,149.6,136.6,133.6,131.9,128.7,128.4,127.9$, $127.0,126.8,126.5,125.3,125.1,20.6,19.2,15.1$.

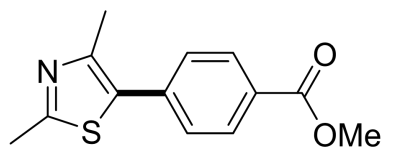

methyl 4-(2,4-dimethylthiazol-5-yl)benzoate (5bt): ${ }^{1} \mathrm{H}$ NMR $(400 \mathrm{MHz}$, $\left.\mathrm{CDCl}_{3}\right) \delta 8.08(\mathrm{~d}, J=2.4 \mathrm{~Hz}, \mathrm{Ar}-\mathrm{H}, 1 \mathrm{H}), 8.06(\mathrm{~d}, J=2.4 \mathrm{~Hz}, \mathrm{Ar}-\mathrm{H}, 1 \mathrm{H})$, 7.50 (d, $J=2.4 \mathrm{~Hz}, \mathrm{Ar}-\mathrm{H}, 1 \mathrm{H}), 7.48$ (d, $J=2.4 \mathrm{~Hz}, \mathrm{Ar}-\mathrm{H}, 1 \mathrm{H}), 3.94$ (d, $J$ $\left.=2.8 \mathrm{~Hz}, \mathrm{COOCH}_{3}, 3 \mathrm{H}\right), 2.70\left(\mathrm{~d}, J=2.7 \mathrm{~Hz}, \mathrm{CH}_{3}, 3 \mathrm{H}\right), 2.49\left(\mathrm{~d}, J=2.7 \mathrm{~Hz}, \mathrm{CH}_{3}, 3 \mathrm{H}\right) .{ }^{13} \mathrm{C} \mathrm{NMR}(101$ $\left.\mathrm{MHz}, \mathrm{CDCl}_{3}\right) \delta 166.6,164.2,148.2,137.1,130.4,129.9,129.0,128.8,19.2,16.3$.

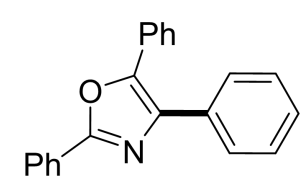

2,4,5-triphenyloxazole (5cf): ${ }^{1} \mathrm{H}$ NMR $\left(400 \mathrm{MHz}, \mathrm{CDCl}_{3}\right) \delta 8.19-8.16(\mathrm{~m}$, Ar-H, 2H), 7.75 (dd, $J=7.1,1.1 \mathrm{~Hz}, \mathrm{Ar}-\mathrm{H}, 2 \mathrm{H}), 7.71-7.68$ (m, Ar-H, 2H), 7.51 -7.47 (m, Ar-H, 3H), $7.43-7.35$ (m, Ar-H, 6H). $\left.{ }^{13} \mathrm{C} \mathrm{NMR} \mathrm{(101} \mathrm{MHz,} \mathrm{CDCl}_{3}\right) \delta$ $160.1,145.5,136.7,132.5,130.3,128.9,128.7,128.7,128.6,128.5,128.2$, $128.1,127.3,126.5,126.4$.<smiles>Clc1ccc(-c2nc(-c3ccccc3)oc2-c2ccccc2)cn1</smiles>

4-(6-chloropyridin-3-yl)-2,5-diphenyloxazole (5cp): ${ }^{1} \mathrm{H}$ NMR (400 MHz, $\left.\mathrm{CDCl}_{3}\right) \delta 8.06(\mathrm{ddd}, J=5.2,4.2,1.9 \mathrm{~Hz}, \mathrm{Ar}-\mathrm{H}, 2 \mathrm{H}), 7.61-7.54$ (m, Ar-H, 4H), $7.41-7.37$ (m, Ar-H, 3H), 7.32 (d, $J=1.4 \mathrm{~Hz}, \mathrm{Ar}-\mathrm{H}, 1 \mathrm{H}), 7.31-7.29$ (m, Ar-H, 2H), 7.28 (d, $J=1.9 \mathrm{~Hz}, \mathrm{Ar}-\mathrm{H}, 1 \mathrm{H}) .{ }^{13} \mathrm{C} \mathrm{NMR}\left(101 \mathrm{MHz}, \mathrm{CDCl}_{3}\right)$ $\delta 160.3,145.8,135.6,134.0,131.0,130.5,129.3,128.8,128.8,128.8,128.7,127.2,126.7,126.4$.<smiles>Cc1ccc(-c2nc(-c3ccccc3)oc2-c2ccccc2)cc1</smiles>

2,5-diphenyl-4-(p-tolyl)oxazole (5cu): ${ }^{1} \mathrm{H}$ NMR (400 MHz, $\left.\mathrm{CDCl}_{3}\right) \delta 8.07$ 
(dd, $J=7.8,1.9 \mathrm{~Hz}, \mathrm{Ar}-\mathrm{H}, 2 \mathrm{H}), 7.63-7.59$ (m, Ar-H, 2H), $7.56-7.52$ (m, Ar-H, 2H), $7.42-7.37$ (m, 3H), $7.33-7.24$ (m, Ar-H, 3H), 7.14 (d, J=7.9 Hz, Ar-H, 2H), 2.32 (s, $\left.\mathrm{CH}_{3}, 3 \mathrm{H}\right) .{ }^{13} \mathrm{C}$ NMR $(101 \mathrm{MHz}$, $\left.\mathrm{CDCl}_{3}\right) \delta 160.0,145.2,138.1,136.9,130.3,129.6,129.3,129.1,128.7,128.6,128.4,128.0,127.4$, 126.5, 126.4, 21.3.

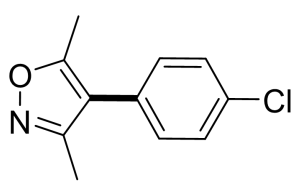

4-(4-chlorophenyl)-3,5-dimethylisoxazole (5de): ${ }^{1} \mathrm{H}$ NMR (400 $\mathrm{MHz}, \mathrm{CDCl}_{3}$ ) $\delta 7.30(\mathrm{dd}, J=6.7,1.2 \mathrm{~Hz}, \mathrm{Ar}-\mathrm{H}, 2 \mathrm{H}), 7.10(\mathrm{~d}, J=8.4 \mathrm{~Hz}, \mathrm{Ar}-\mathrm{H}, 2 \mathrm{H}), 2.28(\mathrm{~d}, J$ $\left.=1.2 \mathrm{~Hz}, \mathrm{CH}_{3}, 3 \mathrm{H}\right), 2.14\left(\mathrm{~d}, J=1.1 \mathrm{~Hz}, \mathrm{CH}_{3}, 3 \mathrm{H}\right) .{ }^{13} \mathrm{C} \mathrm{NMR}\left(101 \mathrm{MHz}, \mathrm{CDCl}_{3}\right)$ $\delta 165.1,158.2,133.3,130.2,128.8,128.7,115.5,11.3,10.5$.

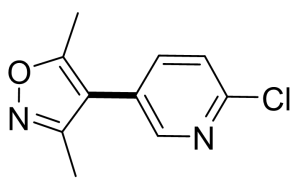

4-(6-chloropyridin-3-yl)-3,5-dimethylisoxazole (5dp): ${ }^{1} \mathrm{H}$ NMR (400 MHz, $\left.\mathrm{CDCl}_{3}\right) \delta 8.33-8.30(\mathrm{~m}, \mathrm{Ar}-\mathrm{H}, 1 \mathrm{H}), 7.56(\mathrm{dd}, J=8.2,2.5 \mathrm{~Hz}, \mathrm{Ar}-\mathrm{H}, 1 \mathrm{H}), 7.43$ $(\mathrm{dd}, J=8.2,0.6 \mathrm{~Hz}, \mathrm{Ar}-\mathrm{H}, 1 \mathrm{H}), 2.42\left(\mathrm{~s}, \mathrm{CH}_{3}, 3 \mathrm{H}\right), 2.27\left(\mathrm{~s}, \mathrm{CH}_{3}, 3 \mathrm{H}\right) .{ }^{13} \mathrm{C} \mathrm{NMR}$ $\left(101 \mathrm{MHz}, \mathrm{CDCl}_{3}\right) \delta 166.3,158.3,150.8,149.6,138.9,125.5,124.5,112.4$,

$11.6,10.7$

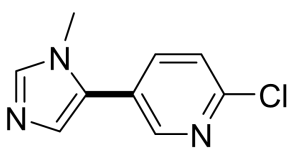

2-chloro-5-(1-methyl-1H-imidazol-5-yl)pyridine (5ep): ${ }^{1} \mathrm{H}$ NMR (400 MHz, $\left.\mathrm{CDCl}_{3}\right) \delta 8.43(\mathrm{~d}, J=2.0 \mathrm{~Hz}, 1 \mathrm{H}), 7.67(\mathrm{dd}, J=8.2,2.5 \mathrm{~Hz}, \mathrm{Ar}-\mathrm{H}, 1 \mathrm{H}), 7.57$ (s, Ar-H, 1H), 7.41 (dd, $J=8.2,0.5 \mathrm{~Hz}, \mathrm{Ar}-\mathrm{H}, 1 \mathrm{H}), 7.16$ (s, Ar-H, 1H), 3.67 (s, $\left.\mathrm{CH}_{3}, 3 \mathrm{H}\right) .{ }^{13} \mathrm{C}$ NMR $\left(101 \mathrm{MHz}, \mathrm{CDCl}_{3}\right) \delta 150.9,148.6,140.2,138.2,129.5,128.7,124.9,124.3,32.6$.

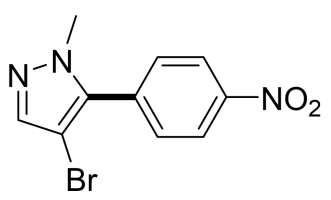

5-bromo-1-methyl-5-(4-nitrophenyl)-1H-pyrazole (5fa): ${ }^{1} \mathrm{H}$ NMR (400 $\left.\mathrm{MHz} \mathrm{CDCl}_{3}\right) \delta 8.39$ (s, Ar-H, 1H), 8.37 (d, $\left.J=2.0 \mathrm{~Hz}, \mathrm{Ar}-\mathrm{H}, 1 \mathrm{H}\right), 7.64$ (d, $J$ $=2.1 \mathrm{~Hz}, \mathrm{Ar}-\mathrm{H}, 1 \mathrm{H}), 7.62(\mathrm{~d}, J=2.0 \mathrm{~Hz}, \mathrm{Ar}-\mathrm{H}, 1 \mathrm{H}), 7.58(\mathrm{~s}, \mathrm{Ar}-\mathrm{H}, 1 \mathrm{H}), 3.86$ $\left(\mathrm{s}, \mathrm{CH}_{3}, 3 \mathrm{H}\right) .{ }^{13} \mathrm{C} \mathrm{NMR}\left(101 \mathrm{MHz}, \mathrm{CDCl}_{3}\right) \delta 148.0,139.7,139.0,134.8$, $130.8,124.0,94.4,38.6$.<smiles>Cn1ncc(Br)c1-c1ccc(C#N)cc1</smiles>

4-(4-bromo-1-methyl-1H-pyrazol-5-yl)benzonitrile (5fb): ${ }^{1} \mathrm{H}$ NMR (400 $\left.\mathrm{MHz} \mathrm{CDCl}_{3}\right) \delta 7.81$ (s, Ar-H, 1H), 7.79 (s, Ar-H, 1H), 7.56 (s, Ar-H, 1H), 7.54 $(\mathrm{d}, J=2.8 \mathrm{~Hz}, \mathrm{Ar}-\mathrm{H}, 2 \mathrm{H}), 3.83\left(\mathrm{~s}, \mathrm{CH}_{3}, 3 \mathrm{H}\right) .{ }^{13} \mathrm{C} \mathrm{NMR}\left(101 \mathrm{MHz}, \mathrm{CDCl}_{3}\right) \delta$ $139.5,139.2,132.9,132.4,130.4,118.1,113.0,94.1,38.5$.

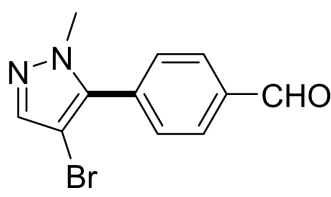

4-(4-bromo-1-methyl-1H-pyrazol-5-yl)benzaldehyde (5fc): ${ }^{1} \mathrm{H}$ NMR (400 $\left.\mathrm{MHz}, \mathrm{CDCl}_{3}\right) \delta 10.10$ (s, CHO, 1H), 8.03 (d, J=1.9 Hz, Ar-H, 1H), 8.02 (d, $J=1.9 \mathrm{~Hz}, \operatorname{Ar}-\mathrm{H}, 1 \mathrm{H}), 7.62$ (d, $J=1.6 \mathrm{~Hz}, \mathrm{Ar}-\mathrm{H}, 1 \mathrm{H}), 7.60$ (d, $J=1.6 \mathrm{~Hz}$, Ar-H, 1H), 7.57 (s, Ar-H, 1H), 3.85 (s, $\left.\mathrm{CH}_{3}, 3 \mathrm{H}\right) .{ }^{13} \mathrm{C} \mathrm{NMR}(101 \mathrm{MHz}$, $\left.\mathrm{CDCl}_{3}\right) \delta 191.4,139.9,139.6,136.4,134.3,130.4,129.9,94.0,38.6$.

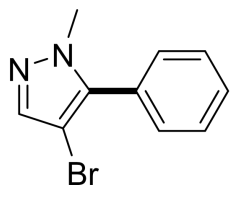

4-bromo-1-methyl-5-phenyl-1H-pyrazole (5ff): ${ }^{1} \mathrm{H}$ NMR (400 $\left.\mathrm{MHz}, \mathrm{CDCl}_{3}\right) \delta$ 7.54 (s, Ar-H, 1H), $7.51-7.46$ (m, Ar-H, 3H), 7.40 (dd, J= 7.9, 1.6 Hz, Ar-H, 2H), $3.82\left(\mathrm{~s}, \mathrm{CH}_{3}, 3 \mathrm{H}\right) .{ }^{13} \mathrm{C} \mathrm{NMR}\left(101 \mathrm{MHz}, \mathrm{CDCl}_{3}\right) \delta 141.2,139.2,129.8,129.2,128.7$, 128.4, 93.4, 38.3. 


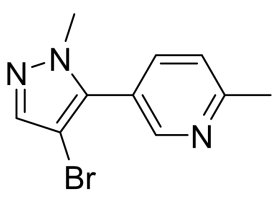

(4-bromo-1-methyl-1H-pyrazol-5-yl)-2-methylpyridine (5fi): ${ }^{1} \mathrm{H}$ NMR (400 $\left.\mathrm{MHz} \mathrm{CDCl}_{3}\right) \delta 8.56(\mathrm{~d}, J=1.9 \mathrm{~Hz}, \mathrm{Ar}-\mathrm{H}, 1 \mathrm{H}), 7.65(\mathrm{dd}, J=8.0,2.3 \mathrm{~Hz}, \mathrm{Ar}-\mathrm{H}$, 1H), 7.55 (s, Ar-H, 1H), 7.32 (d, $J=7.8 \mathrm{~Hz}, \mathrm{Ar}-\mathrm{H}, 1 \mathrm{H}), 3.84$ (s, $\left.\mathrm{CH}_{3}, 3 \mathrm{H}\right), 2.65$ $\left(\mathrm{s}, \mathrm{CH}_{3}, 3 \mathrm{H}\right) .{ }^{13} \mathrm{C} \mathrm{NMR}\left(101 \mathrm{MHz}, \mathrm{CDCl}_{3}\right) \delta 159.3,149.4,139.3,138.1,137.3$, 123.0, 121.6, 94.1, 38.3, 24.3.

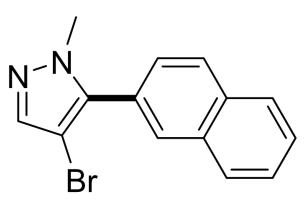

4-bromo-1-methyl-5-(naphthalen-2-yl)-1H-pyrazole (5fr): ${ }^{1} \mathrm{H}$ NMR (400 $\left.\mathrm{MHz} \mathrm{CDCl}_{3}\right) \delta 7.97(\mathrm{~d}, J=8.4 \mathrm{~Hz}, \mathrm{Ar}-\mathrm{H}, 1 \mathrm{H}), 7.91$ (dd, $J=8.3,3.8 \mathrm{~Hz}, \mathrm{Ar}-\mathrm{H}$, $3 \mathrm{H}), 7.57$ (dd, $J=7.4,3.5 \mathrm{~Hz}, \mathrm{Ar}-\mathrm{H}, 3 \mathrm{H}), 7.49$ (dd, $J=8.4,1.7 \mathrm{~Hz}, \mathrm{Ar}-\mathrm{H}, 1 \mathrm{H})$, $3.87\left(\mathrm{~s}, \mathrm{CH}_{3}, \mathrm{H}\right) .{ }^{13} \mathrm{C}$ NMR $\left(101 \mathrm{MHz}, \mathrm{CDCl}_{3}\right) \delta 141.2,139.3,133.2,133.0$, $129.6,128.5,128.2,127.8,127.1,126.8,126.7,125.7,93.7,38.4$.

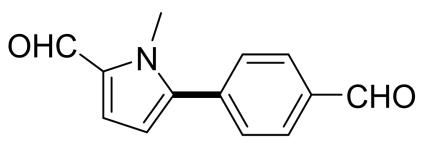

5-(4-formylphenyl)-1-methyl-1H-pyrrole-2-carbaldehyde (5gc): ${ }^{1} \mathrm{H}$ NMR (400 MHz, $\left.\mathrm{CDCl}_{3}\right) \delta 10.08(\mathrm{~s}, \mathrm{CHO}, 1 \mathrm{H}), 9.63(\mathrm{~s}, \mathrm{CHO}, 1 \mathrm{H})$, $8.00-7.96$ (m, Ar-H, 2H), $7.64-7.59$ (m, Ar-H, 2H), 7.01 (d, $J=4.1$

$\mathrm{Hz}, \mathrm{Ar}-\mathrm{H}, 1 \mathrm{H}), 6.41$ (d, $J=4.1 \mathrm{~Hz}, \mathrm{Ar}-\mathrm{H}, 1 \mathrm{H}), 3.97$ (s, $\left.\mathrm{CH}_{3}, 3 \mathrm{H}\right) .{ }^{13} \mathrm{C} \mathrm{NMR}\left(101 \mathrm{MHz}, \mathrm{CDCl}_{3}\right) \delta 191.5$, $178.0,142.5,136.9,135.9,133.8,129.9,129.6,124.3,111.6,34.6$.<smiles>Cn1c(C=O)ccc1-c1cccnc1</smiles>

1-methyl-5-(pyridin-3-yl)-1H-pyrrole-2-carbaldehyde (5gj): ${ }^{1} \mathrm{H}$ NMR (400 $\left.\mathrm{MHz} \mathrm{CDCl}_{3}\right) \delta 9.61(\mathrm{~s}, \mathrm{CHO}, 1 \mathrm{H}), 8.71(\mathrm{~d}, J=1.6 \mathrm{~Hz}, \mathrm{Ar}-\mathrm{H}, 1 \mathrm{H}), 8.66$ (dd, $J$ $=4.9,1.6 \mathrm{~Hz}, \mathrm{Ar}-\mathrm{H}, 1 \mathrm{H}), 7.76-7.73(\mathrm{~m}, \mathrm{Ar}-\mathrm{H}, 1 \mathrm{H}), 7.41$ (ddd, J= 7.9, 4.9, $0.7 \mathrm{~Hz}, \mathrm{Ar}-\mathrm{H}, 1 \mathrm{H}), 7.01$ (d, $J=4.1 \mathrm{~Hz}, \mathrm{Ar}-\mathrm{H}, 1 \mathrm{H}), 6.36(\mathrm{~d}, J=4.1 \mathrm{~Hz}, \mathrm{Ar}-\mathrm{H}, 1 \mathrm{H}), 3.94\left(\mathrm{~s}, \mathrm{CH}_{3}, 3 \mathrm{H}\right) .{ }^{13} \mathrm{C} \mathrm{NMR}\left(101 \mathrm{MHz}, \mathrm{CDCl}_{3}\right)$ $\delta 179.9,149.8,149.6,140.3,136.3,133.6,127.3,124.3,123.4,111.3,34.3$.<smiles>Cn1c(C=O)ccc1-c1cncnc1</smiles>

(pyrimidin-5-yl)-1H-pyrrole-2-carbaldehyde (5gq): ${ }^{1} \mathrm{H}$ NMR $(400 \mathrm{MHz}$, $\left.\mathrm{CDCl}_{3}\right) \delta 9.65(\mathrm{~s}, \mathrm{CHO}, 1 \mathrm{H}), 9.25(\mathrm{~s}, \mathrm{Ar}-\mathrm{H}, 1 \mathrm{H}), 8.83(\mathrm{~s}, \mathrm{Ar}-\mathrm{H}, 2 \mathrm{H}), 7.03(\mathrm{~d}, J$ $=4.1 \mathrm{~Hz}, \mathrm{Ar}-\mathrm{H}, 1 \mathrm{H}), 6.42(\mathrm{~d}, J=4.1 \mathrm{~Hz}, \mathrm{Ar}-\mathrm{H}, 1 \mathrm{H}), 3.96\left(\mathrm{~s}, \mathrm{CH}_{3}, 3 \mathrm{H}\right) .{ }^{13} \mathrm{C}$ NMR $\left(101 \mathrm{MHz}, \mathrm{CDCl}_{3}\right) \delta 180.1,158.2,156.4,136.4,134.2,125.8,124.2,111.9,34.3$.

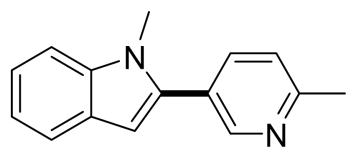

1-methyl-2-(6-methylpyridin-3-yl)-1H-indole (5hi): ${ }^{1} \mathrm{H}$ NMR (400 MHz, $\left.\mathrm{CDCl}_{3}\right) \delta 8.59(\mathrm{~d}, J=2.2 \mathrm{~Hz}, \mathrm{Ar}-\mathrm{H}, 1 \mathrm{H}), 7.63-7.53$ (m, Ar-H, 2H), 7.28 (dd, $J=8.2,0.7 \mathrm{~Hz}, \mathrm{Ar}-\mathrm{H}, 1 \mathrm{H}), 7.18$ (dd, $J=12.0,4.5 \mathrm{~Hz}, \mathrm{Ar}-\mathrm{H}, 2 \mathrm{H}), 7.07$ $(\mathrm{dd}, J=7.7,7.2 \mathrm{~Hz}, \mathrm{Ar}-\mathrm{H}, 1 \mathrm{H}), 6.51(\mathrm{~s}, \mathrm{Ar}-\mathrm{H}, 1 \mathrm{H}), 3.64\left(\mathrm{~s}, \mathrm{CH}_{3}, 3 \mathrm{H}\right), 2.55\left(\mathrm{~s}, \mathrm{CH}_{3}, 3 \mathrm{H}\right) .{ }^{13} \mathrm{C} \mathrm{NMR}(101$ $\left.\mathrm{MHz}_{,} \mathrm{CDCl}_{3}\right) \delta 157.8,149.2,138.4,137.9,136.7,127.8,125.8,122.9,122.0,120.5,120.0,109.6$, $102.3,31.0,24.3$.

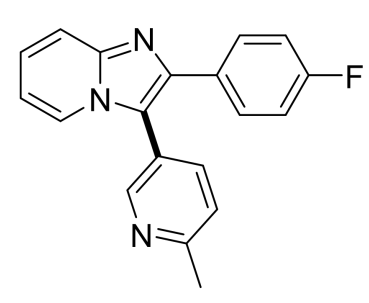

2-(4-fluorophenyl)-3-(6-methylpyridin-3-yl)imidazo[1,2-a]pyridine (5ii): ${ }^{1} \mathrm{H}$ NMR $\left(400 \mathrm{MHz}, \mathrm{CDCl}_{3}\right) \delta 8.62(\mathrm{~d}, J=1.7 \mathrm{~Hz}, \mathrm{Ar}-\mathrm{H}, 1 \mathrm{H}), 7.93$ (dt, $J=$ 6.9, 1.1 Hz, Ar-H, 1H), 7.71 (dt, $J=9.1,1.1 \mathrm{~Hz}, \mathrm{Ar}-\mathrm{H}, 1 \mathrm{H}), 7.66-7.59$ (m, Ar-H, 3H), 7.34 (d, J=8.0 Hz, Ar-H, 1H), $7.26-7.22$ (m, Ar-H, 1H), 7.03 -6.98 (m, Ar-H, 2H), 6.80 (td, $J=6.8,1.2 \mathrm{~Hz}, \mathrm{Ar}-\mathrm{H}, 1 \mathrm{H}), 2.69$ (s, $\mathrm{CH}_{3}$, $3 \mathrm{H}) .{ }^{13} \mathrm{C} \mathrm{NMR}\left(101 \mathrm{MHz}, \mathrm{CDCl}_{3}\right) \delta 159.2,150.6,145.2,142.7,138.6$, $129.9,129.8,125.3,124.0,123.0,123.0,117.7,117.4,115.6,115.4,112.8,24.4$. 


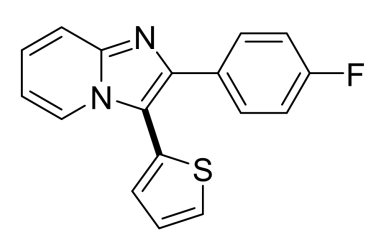

2-(4-fluorophenyl)-3-(thiophen-2-yl)imidazo[1,2-a]pyridine $\quad$ (5iv): ${ }^{1} \mathrm{H}$ NMR (400 MHz, $\left.\mathrm{CDCl}_{3}\right) \delta 7.97(\mathrm{dt}, J=6.9,1.1 \mathrm{~Hz}, \mathrm{Ar}-\mathrm{H}, 1 \mathrm{H}), 7.74-7.65$ (m, Ar-H, 3H), 7.59 (dd, $J=5.2,1.2 \mathrm{~Hz}, \mathrm{Ar}-\mathrm{H}, 1 \mathrm{H}), 7.25-7.20$ (m, Ar-H, 3H), 7.00 (ddd, $J=10.7,6.0,2.5 \mathrm{~Hz}, \mathrm{Ar}-\mathrm{H}, 2 \mathrm{H}), 6.79$ (td, $J=6.8,1.1 \mathrm{~Hz}$, $\mathrm{Ar}-\mathrm{H}, 1 \mathrm{H}) .{ }^{13} \mathrm{C} \mathrm{NMR}\left(101 \mathrm{MHz}, \mathrm{CDCl}_{3}\right) \delta 163.8,161.3,145.3,143.6,130.3$, $129.9,129.8,129.8,129.7,129.0,128.2$, 125.4, 123.9, 117.3, 115.4, 115.2, 113.1, 112.7.

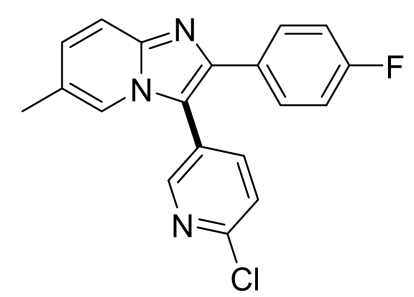

3-(6-chloropyridin-3-yl)-2-(4-fluorophenyl)-6-methylimidazo[1,2-a]p yridine(5jp): ${ }^{1} \mathrm{H}$ NMR $\left(400 \mathrm{MHz}, \mathrm{CDCl}_{3}\right) \delta 8.50(\mathrm{~d}, J=2.3 \mathrm{~Hz}, \mathrm{Ar}-\mathrm{H}$, $1 \mathrm{H}), 7.70(\mathrm{dd}, J=8.2,2.5 \mathrm{~Hz}, \mathrm{Ar}-\mathrm{H}, 2 \mathrm{H}), 7.60$ (d, $J=9.2 \mathrm{~Hz}, \mathrm{Ar}-\mathrm{H}, 1 \mathrm{H})$, $7.57-7.53$ (m, Ar-H, 2H), $7.50(\mathrm{~d}, J=8.2 \mathrm{~Hz}, \mathrm{Ar}-\mathrm{H}, 1 \mathrm{H}), 7.12$ (dd, $J=$ 9.2, 1.5 Hz, Ar-H, 1H), 7.01 (ddd, $J=10.6,6.0,2.5 \mathrm{~Hz}$, Ar-H, 2H), 2.30 $\left(\mathrm{d}, J=0.6 \mathrm{~Hz}, \mathrm{CH}_{3}, 3 \mathrm{H}\right) \cdot{ }^{13} \mathrm{C} \mathrm{NMR}\left(101 \mathrm{MHz}, \mathrm{CDCl}_{3}\right) \delta 163.8,161.4$, $151.7,151.1,144.6,143.1,140.8,129.8,129.8,129.6,129.6,128.8,125.2,123.0,120.3,117.2$, 115.6, $115.5,18.3$

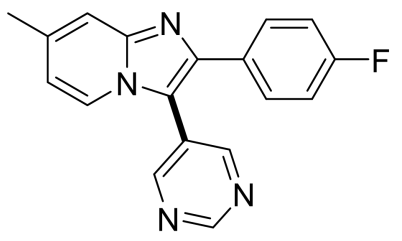

2-(4-fluorophenyl)-7-methyl-3-(pyrimidin-5-yl)imidazo[1,2-a]pyridi ne (5kq): ${ }^{1} \mathrm{H}$ NMR (400 MHz, $\left.\mathrm{CDCl}_{3}\right) \delta 9.27$ (s, Ar-H, 1H), $8.81(\mathrm{~s}$, Ar-H, 2H), 7.87 - 7.84 (m, Ar-H, 1H), $7.54-7.50$ (m, Ar-H, 2H), 7.45 $(\mathrm{dt}, J=1.8,1.0 \mathrm{~Hz}, \mathrm{Ar}-\mathrm{H}, 1 \mathrm{H}), 7.03-6.98$ (m, Ar-H, 2H), 6.68 (dd, $J=$ 7.1, $1.6 \mathrm{~Hz}, \mathrm{Ar}-\mathrm{H}, 1 \mathrm{H}), 2.43$ (d, $\left.J=0.9 \mathrm{~Hz}, \mathrm{CH}_{3}, 3 \mathrm{H}\right) .{ }^{13} \mathrm{C}$ NMR (101 $\left.\mathrm{MHz}, \mathrm{CDCl}_{3}\right) \delta 163.9,161.4,158.1,158.0,146.4,144.0,137.1,130.0,129.9,129.4,129.4,125.1$, 121.6, 116.4, 116.0, 115.8, 115.6, 113.2, 21.3.

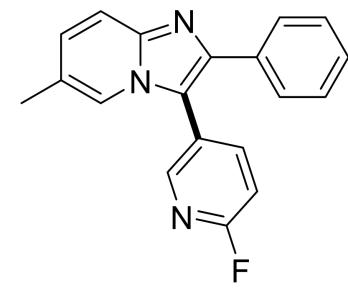

3-(6-fluoropyridin-3-yl)-6-methyl-2-phenylimidazo[1,2-a]pyridine (5lh): ${ }^{1} \mathrm{H}$ NMR $\left(400 \mathrm{MHz}, \mathrm{CDCl}_{3}\right) \delta 8.34(\mathrm{~d}, J=2.4 \mathrm{~Hz}, \mathrm{Ar}-\mathrm{H}, 1 \mathrm{H}), 7.84$ (ddd, $J=$ 8.3, 7.8, 2.5 Hz, Ar-H, 1H), 7.67 (s, Ar-H, 1H), 7.62 - 7.56 (m, Ar-H, 3H), $7.33-7.26$ (m, Ar-H, 3H), 7.11 (dd, $J=8.5,2.6 \mathrm{~Hz}, \mathrm{Ar}-\mathrm{H}, 2 \mathrm{H}), 2.29$ (d, $J=$ $\left.0.7 \mathrm{~Hz}, \mathrm{CH}_{3}, 3 \mathrm{H}\right) .{ }^{13} \mathrm{C} \mathrm{NMR}\left(101 \mathrm{MHz}, \mathrm{CDCl}_{3}\right) \delta 164.7,162.2,149.6,149.5$, $144.5,143.8,143.8,143.7,133.6,128.5,128.0,127.9,124.4,124.4,122.7$,

$120.3,117.2,110.9,110.5,18.3$

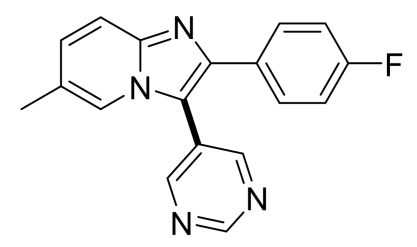

2-(4-fluorophenyl)-6-methyl-3-(pyrimidin-5-yl)imidazo[1,2-a]pyridi ne (5lq): ${ }^{1} \mathrm{H} \mathrm{NMR}\left(400 \mathrm{MHz}, \mathrm{CDCl}_{3}\right) \delta 9.29$ (s, 1H), 8.82 (s, Ar-H, 2H), $7.75-7.72$ (m, Ar-H, 1H), 7.61 (d, $J=9.2 \mathrm{~Hz}, \mathrm{Ar}-\mathrm{H}, 1 \mathrm{H}), 7.54-7.49$ (m, Ar-H, 2H), 7.14 (dd, $J=9.2,1.5 \mathrm{~Hz}, \mathrm{Ar}-\mathrm{H}, 1 \mathrm{H}), 7.04-6.97$ (m, Ar-H, 2H), 2.30 (d, $\left.J=0.8 \mathrm{~Hz}, \mathrm{CH}_{3}, 3 \mathrm{H}\right) \cdot{ }^{13} \mathrm{C} \mathrm{NMR}\left(101 \mathrm{MHz}, \mathrm{CDCl}_{3}\right) \delta$ 163.9, 161.4, 158.2, 158.2, 145.0, 144.0, 129.9, 129.9, 129.4, 129.4, 129.1, 125.1, 123.3, 120.0, 117.3, $115.8,115.6,113.4,18.3$.

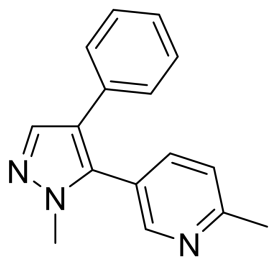

2-methyl-5-(1-methyl-4-phenyl-1H-pyrazol-5-yl)pyridine (7): ${ }^{1} \mathrm{H}$ NMR (400 $\left.\mathrm{MHz} \mathrm{CDCl}_{3}\right) \delta 8.48(\mathrm{~d}, J=1.9 \mathrm{~Hz}, \mathrm{Ar}-\mathrm{H}, 1 \mathrm{H}), 7.73$ (s, Ar-H, 1H), 7.49 (dd, $J=$ 8.0, $2.3 \mathrm{~Hz}, \mathrm{Ar}-\mathrm{H}, 1 \mathrm{H}), 7.24-7.20$ (m, Ar-H, 3H), 7.20 - 7.17 (m, Ar-H, 1H), $7.15(\mathrm{dd}, J=6.8,1.6 \mathrm{~Hz}, \mathrm{Ar}-\mathrm{H}, 2 \mathrm{H}), 3.80\left(\mathrm{~s}, \mathrm{CH}_{3}, 3 \mathrm{H}\right), 2.63\left(\mathrm{~s}, \mathrm{CH}_{3}, 3 \mathrm{H}\right) .{ }^{13} \mathrm{C}$ 
NMR $\left(101 \mathrm{MHz}, \mathrm{CDCl}_{3}\right) \delta 159.0,149.9,137.9,137.9,136.6,132.6,128.6,127.6,126.4,123.6,123.3$, $122.1,37.4,24.4$.

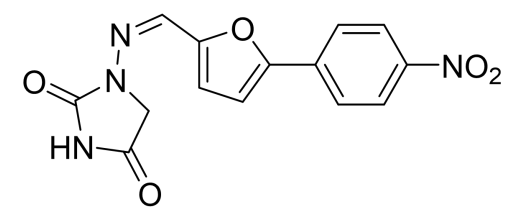

Dantrolene: ${ }^{1} \mathrm{H}$ NMR (400 MHz, DMSO) $\delta 11.36$ (s, NH, 1H), $8.32-8.28(\mathrm{~m}, \mathrm{Ar}-\mathrm{H}, 2 \mathrm{H}), 8.01-7.97(\mathrm{~m}, \mathrm{Ar}-\mathrm{H}, 2 \mathrm{H}), 7.76(\mathrm{~s}$, $\mathrm{N}=\mathrm{CH}, 1 \mathrm{H}), 7.44(\mathrm{~d}, J=3.6 \mathrm{~Hz}, \mathrm{Ar}-\mathrm{H}, 1 \mathrm{H}), 7.03(\mathrm{~d}, J=3.7 \mathrm{~Hz}$, Ar-H, $1 \mathrm{H}), 4.36\left(\mathrm{~s}, \mathrm{CH}_{2}, 2 \mathrm{H}\right){ }^{13} \mathrm{C}$ NMR $(101 \mathrm{MHz}, \mathrm{DMSO}) \delta$ $168.9,153.4,152.2,151.1,146.3,135.2,132.7,124.6,124.6,115.7,112.5,49.0$. 
NMR spectrums of the ligands and palladium complexes

Figure S1. The ${ }^{1} \mathrm{H}$ NMR spectrums of

[2,6-(CHPh $\left.)_{2}-4-\left(\mathrm{CH}_{3}\right)-\mathrm{C}_{6} \mathrm{H}_{2}-\mathrm{N}=\mathrm{C}-(\mathrm{An})-(\mathrm{An})-\mathrm{C}=\mathrm{N}-\left(\mathbf{2}, 4,6-\left(\mathrm{CH}_{3}\right)_{3}-\mathrm{C}_{6} \mathrm{H}_{2}\right)\right](\mathrm{B} 1)$
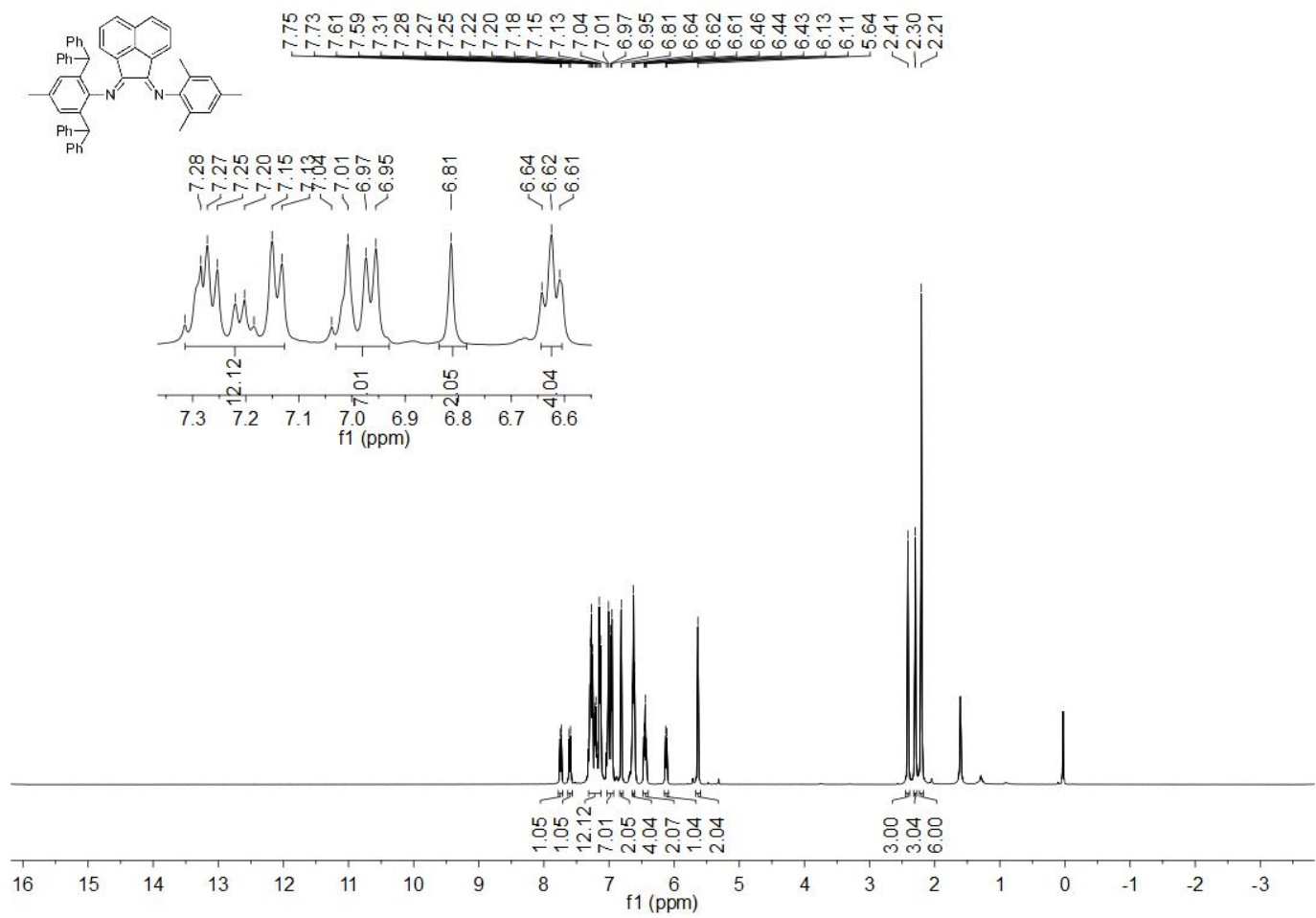

Figure S2. The ${ }^{13} \mathrm{C}$ NMR spectrums of

$\left[2,6-\left(\mathrm{CHPh}_{2}\right)_{2}-4-\left(\mathrm{CH}_{3}\right)-\mathrm{C}_{6} \mathrm{H}_{2}-\mathrm{N}=\mathrm{C}-(\mathrm{An})-(\mathrm{An})-\mathrm{C}=\mathrm{N}-\left(2,4,6-\left(\mathrm{CH}_{3}\right)_{3}-\mathrm{C}_{6} \mathrm{H}_{2}\right)\right](\mathrm{B} 1)$

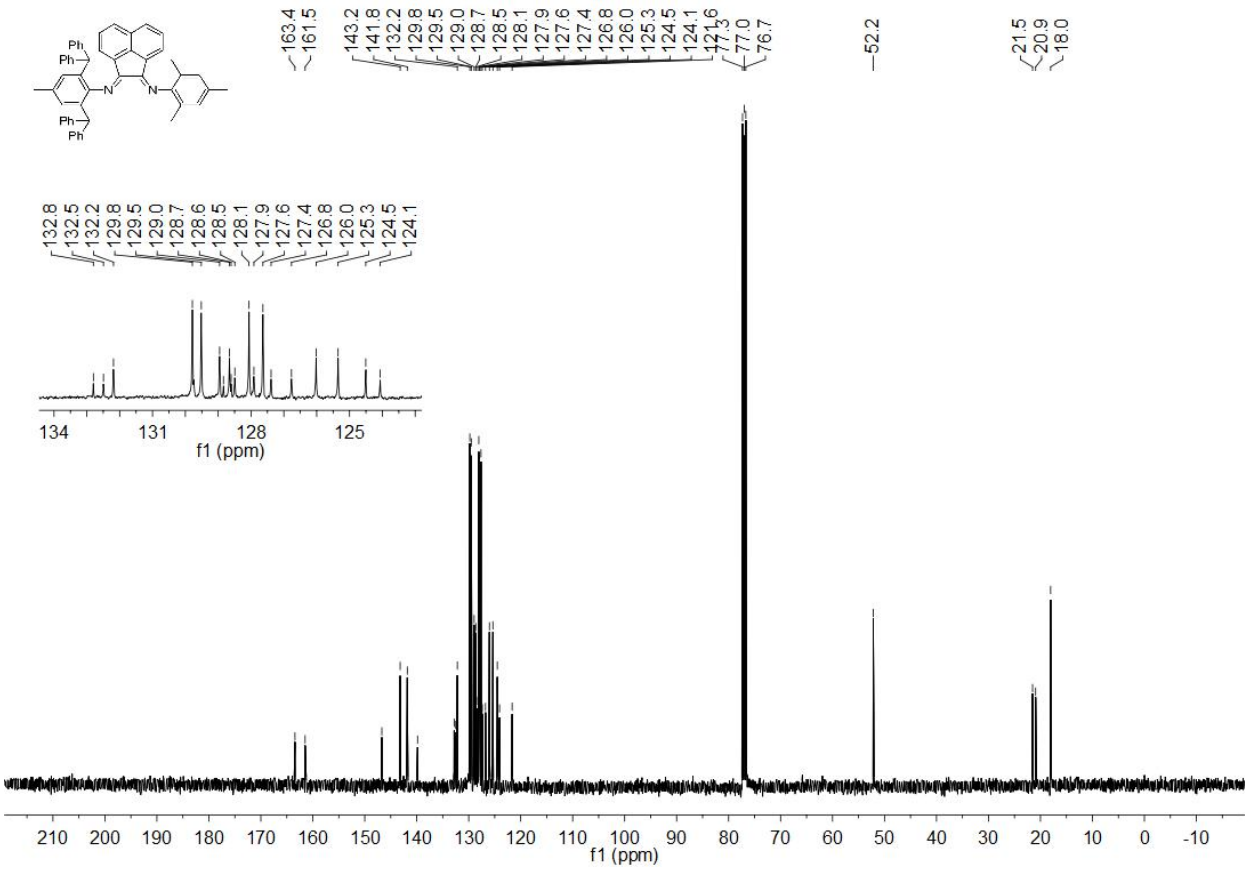


Figure S3. The ${ }^{1} \mathrm{H}$ NMR spectrums of

$\left[2,6-\left(\mathrm{CHPh}_{2}\right)_{2}-4-\left(\mathrm{CH}_{3}\right)-\mathrm{C}_{6} \mathrm{H}_{2}\right]-\left\{[\mathrm{N}=\mathrm{C}-(\mathrm{An})-(\mathrm{An})-\mathrm{C}=\mathrm{N}] \mathrm{CH}^{+} \mathrm{Cl}^{-}\right\}-\left[\left(2,4,6-\left(\mathrm{CH}_{3}\right)_{3}-\mathrm{C}_{6} \mathrm{H}_{2}\right)\right](\mathrm{L1})$

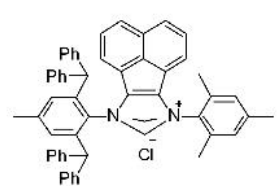

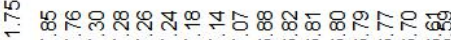

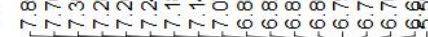

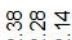

Nitid

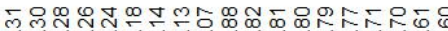

TrTAnTh

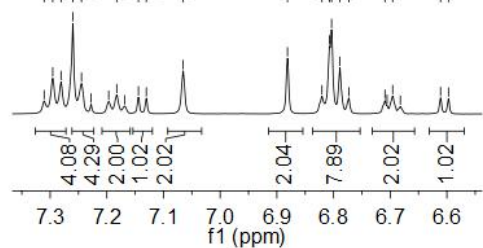

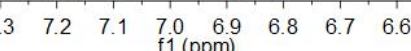

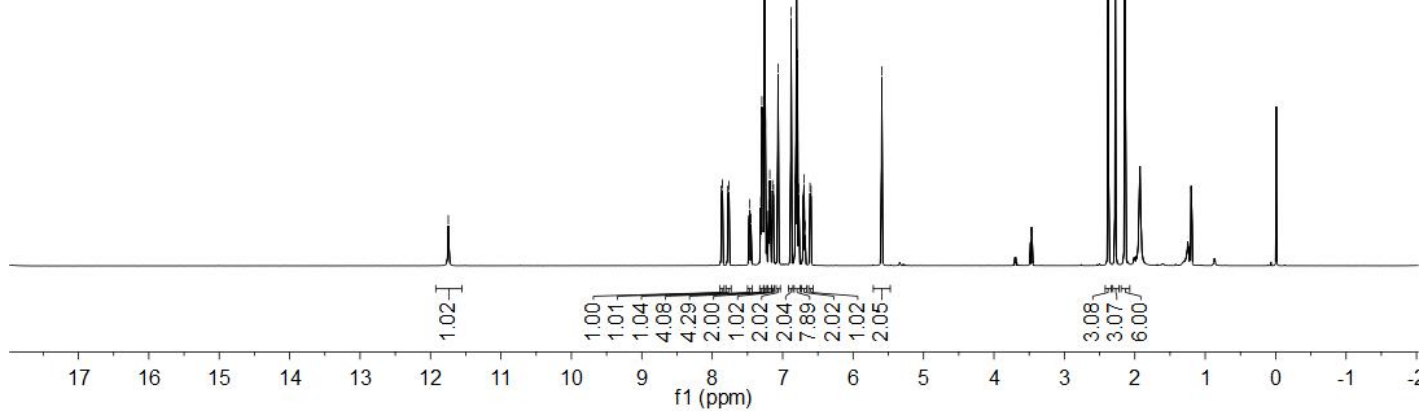

Figure S4. The ${ }^{13} \mathrm{C}$ NMR spectrums of

$\left[2,6-\left(\mathrm{CHPh}_{2}\right)_{2}-4-\left(\mathrm{CH}_{3}\right)-\mathrm{C}_{6} \mathrm{H}_{2}\right]-\left\{[\mathrm{N}=\mathrm{C}-(\mathrm{An})-(\mathrm{An})-\mathrm{C}=\mathrm{N}] \mathrm{CH}^{+} \mathrm{Cl}^{-}\right\}-\left[\left(2,4,6-\left(\mathrm{CH}_{3}\right)_{3}-\mathrm{C}_{6} \mathrm{H}_{2}\right)\right](\mathrm{L1})$
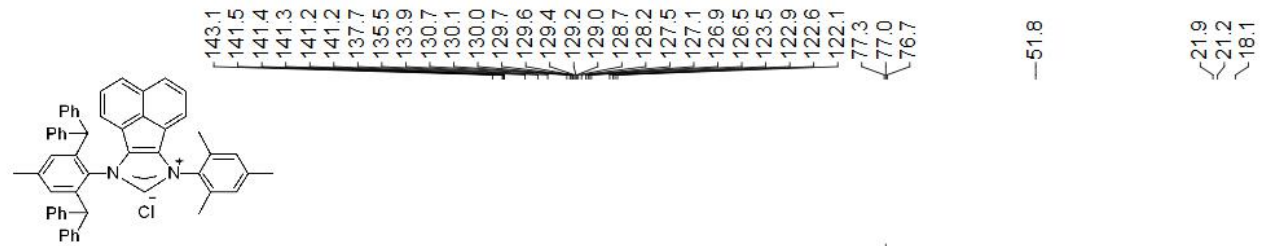

rédn

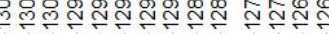
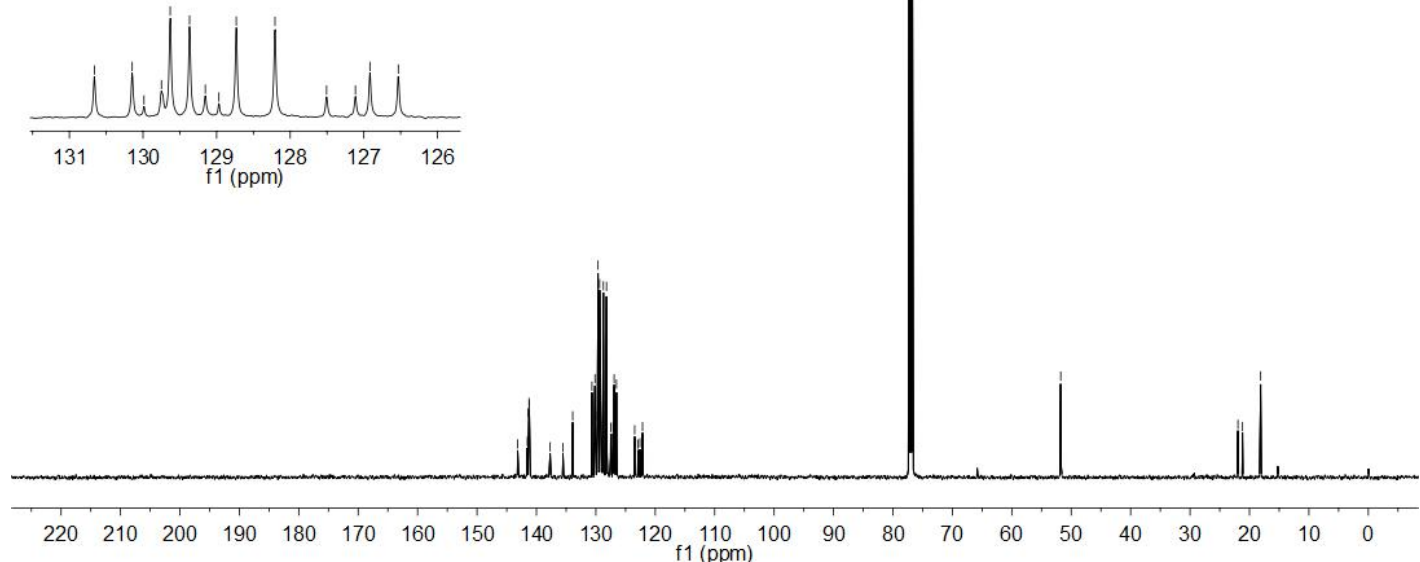
Figure S5. The ${ }^{1} \mathrm{H}$ NMR spectrums of [Pd(IPr*IMe) ${ }^{\mathrm{An}}\left(3-\mathrm{Cl}-\mathrm{py}\right.$ ridinyl)Cl $\left.\mathrm{Cl}_{2}\right](\mathrm{C1})$

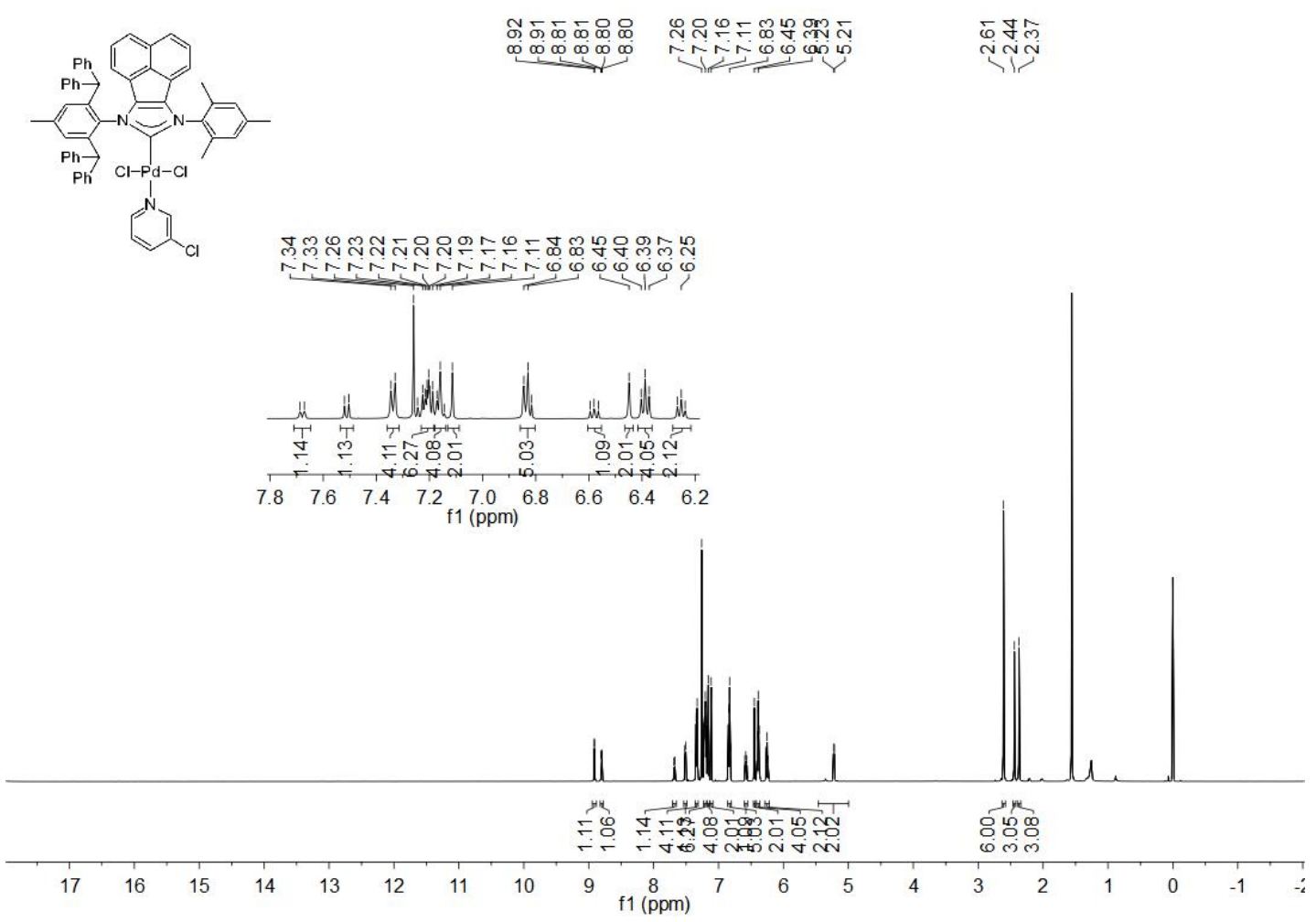

Figure S6. The ${ }^{13} \mathrm{C}$ NMR spectrums of [Pd(IPr*IMe) $\left.{ }^{\mathrm{An}}(3-\mathrm{Cl}-\mathrm{pyridinyl}) \mathrm{Cl}_{2}\right](\mathrm{C1})$

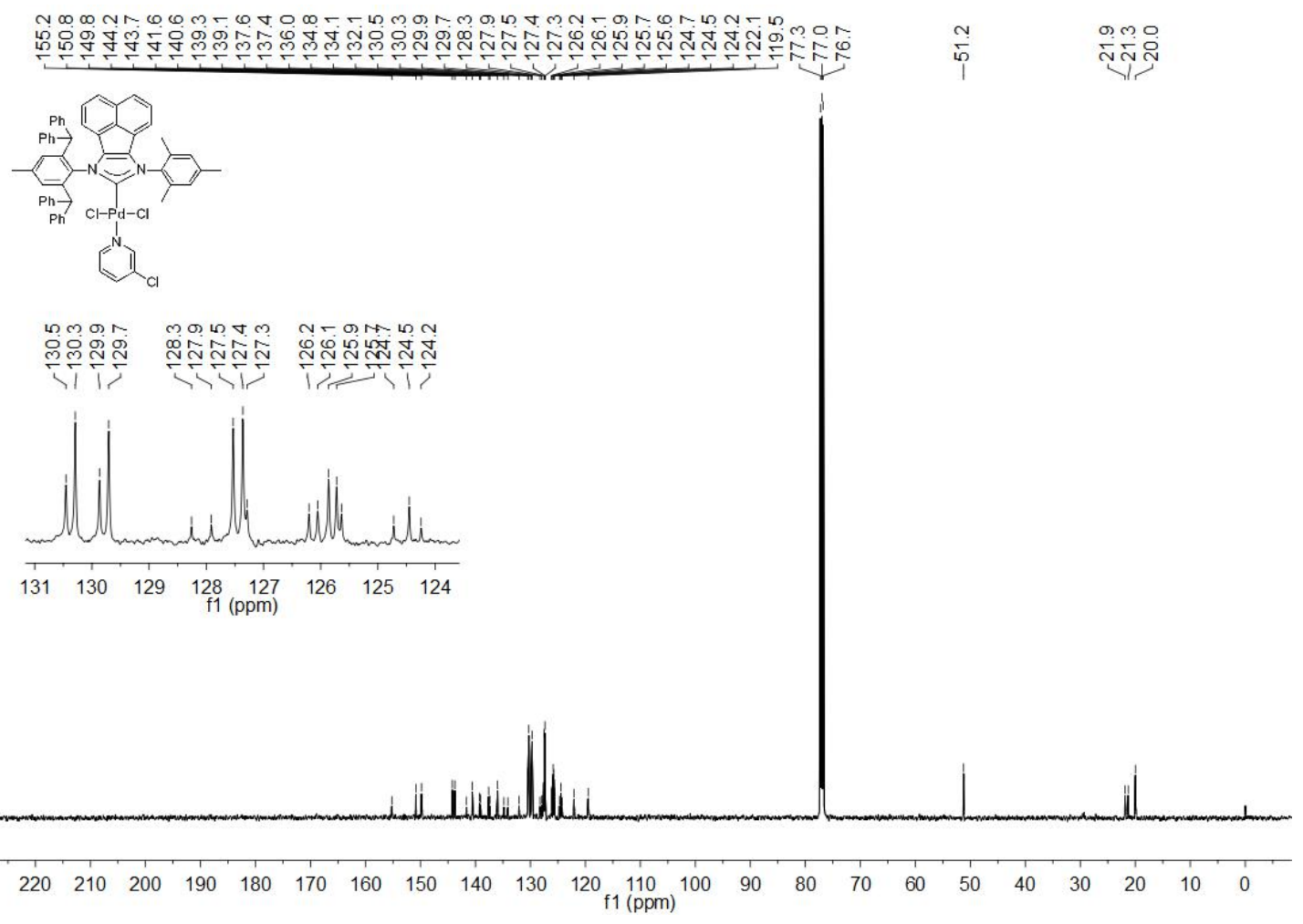


Figure S7. The ${ }^{1} \mathrm{H}$ NMR spectrums of

$\left[2,6-\left(\mathrm{CHPh}_{2}\right)_{2}-4-\left(\mathrm{CH}_{3} \mathrm{O}\right)-\mathrm{C}_{6} \mathrm{H}_{2}-\mathrm{N}=\mathrm{C}-(\mathrm{An})-(\mathrm{An})-\mathrm{C}=\mathrm{N}-\left(2,4,6-\left(\mathrm{CH}_{3}\right)_{3}-\mathrm{C}_{6} \mathrm{H}_{2}\right)\right](\mathrm{B} 2)$

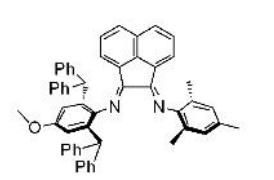

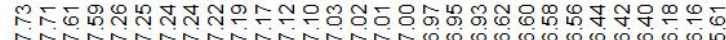

LN

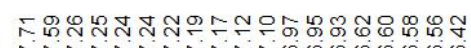

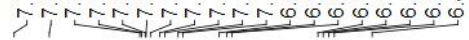
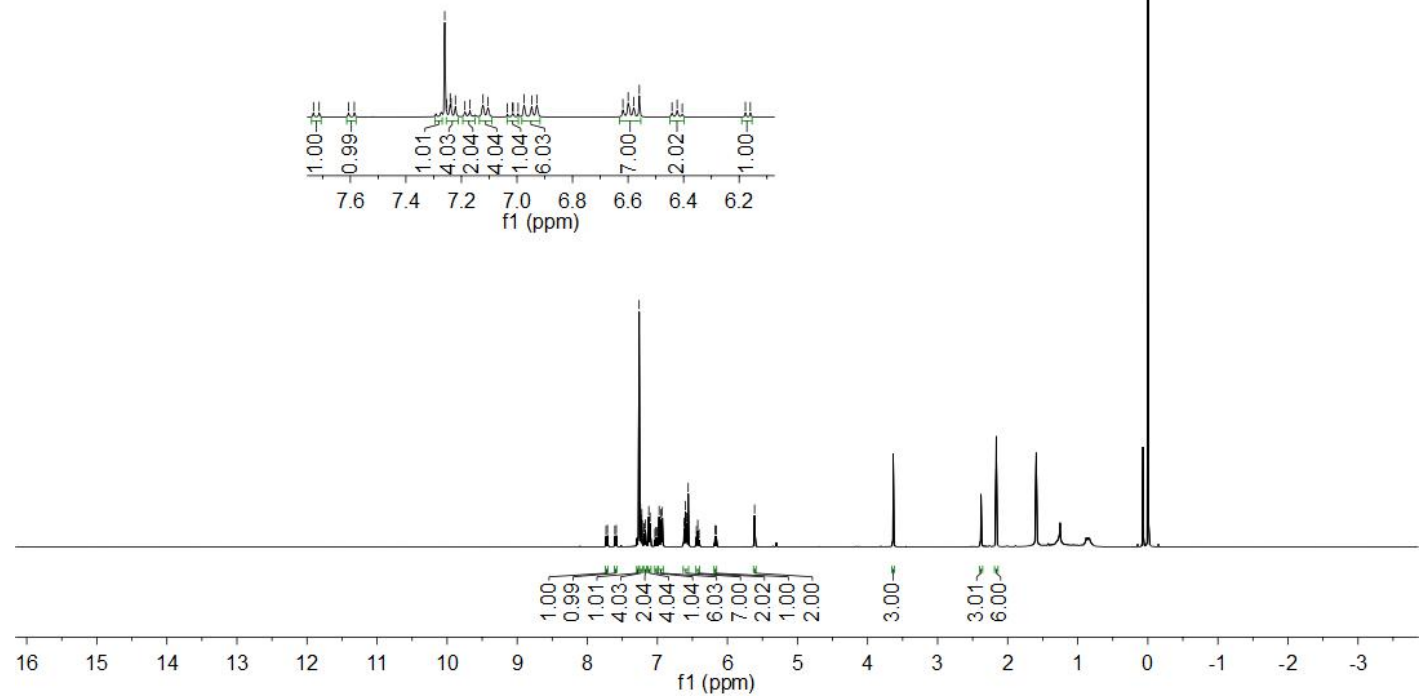

Figure S8. The ${ }^{1} \mathrm{H}$ NMR spectrums of

$\left[2,6-\left(\mathrm{CHPh}_{2}\right)_{2}-4-\left(\mathrm{CH}_{3} \mathrm{O}\right)-\mathrm{C}_{6} \mathrm{H}_{2}-\mathrm{N}=\mathrm{C}-(\mathrm{An})-(\mathrm{An})-\mathrm{C}=\mathrm{N}-\left(\mathbf{2 , 4 , 6}-\left(\mathrm{CH}_{3}\right)_{3}-\mathrm{C}_{6} \mathrm{H}_{2}\right)\right](\mathrm{B2})$
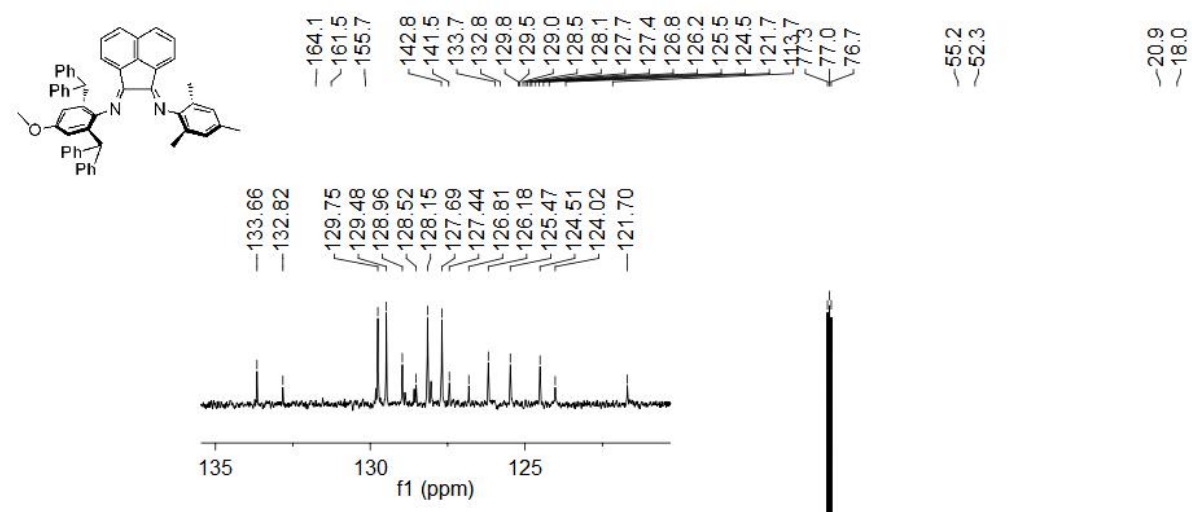

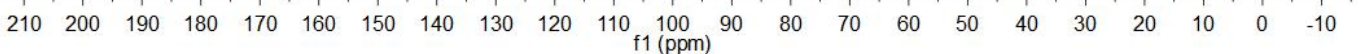


$\begin{array}{lllllll}\text { Figure } & \text { S9. } & \text { The } & { }^{1} \mathbf{H} & \text { NMR } & \text { spectrums } & \text { of }\end{array}$

$\left[2,6-\left(\mathrm{CHPh}_{2}\right)_{2}-4-\left(\mathrm{OCH}_{3}\right)-\mathrm{C}_{6} \mathrm{H}_{2}\right]-\left\{[\mathrm{N}=\mathrm{C}-(\mathrm{An})-(\mathrm{An})-\mathrm{C}=\mathrm{N}] \mathrm{CH}^{+} \mathrm{Cl}^{-}\right\}-\left[\left(2,4,6-\left(\mathrm{CH}_{3}\right)_{3}-\mathrm{C}_{6} \mathrm{H}_{3}\right)\right](\mathrm{L2})$

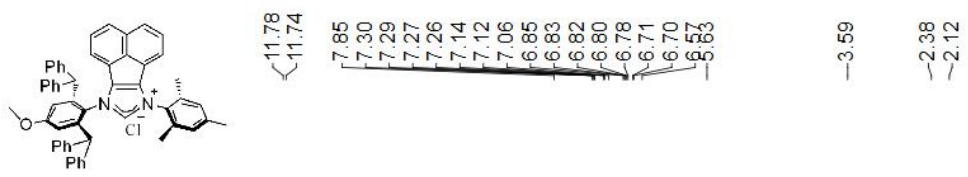

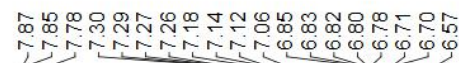
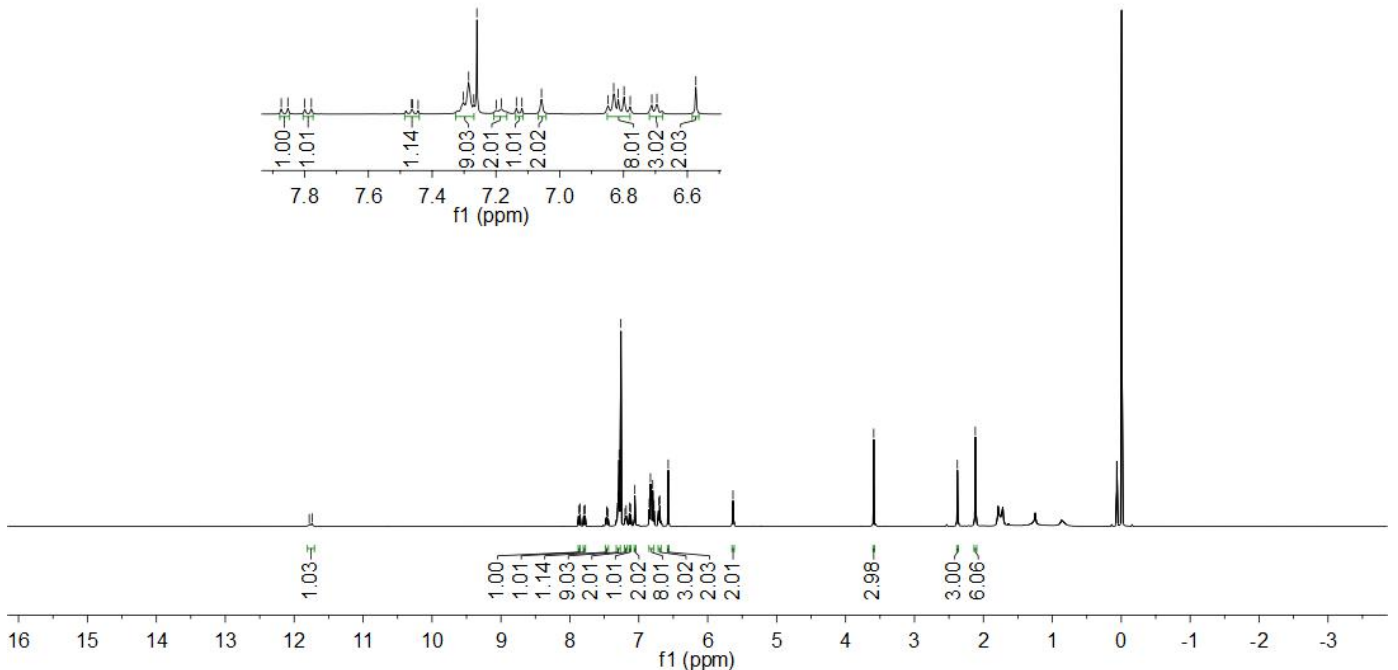

Figure S10. The ${ }^{13} \mathrm{C}$ NMR spectrums of

$\left[2,6-\left(\mathrm{CHPh}_{2}\right)_{2}-4-\left(\mathrm{OCH}_{3}\right)-\mathrm{C}_{6} \mathrm{H}_{2}\right]-\left\{[\mathrm{N}=\mathrm{C}-(\mathrm{An})-(\mathrm{An})-\mathrm{C}=\mathrm{N}] \mathrm{CH}^{+} \mathrm{Cl}^{-}\right\}-\left[\left(2,4,6-\left(\mathrm{CH}_{3}\right)_{3}-\mathrm{C}_{6} \mathrm{H}_{3}\right)\right](\mathrm{L2})$

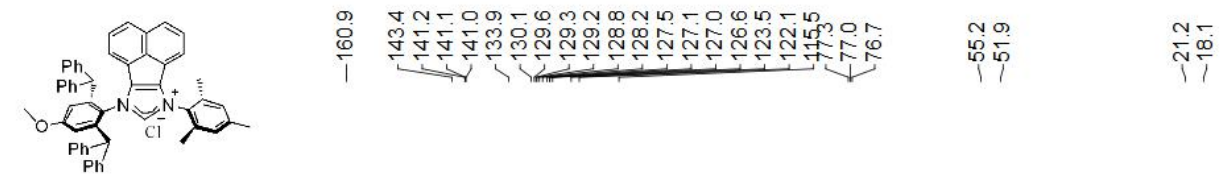

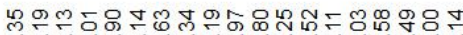

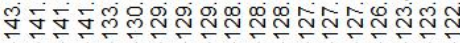

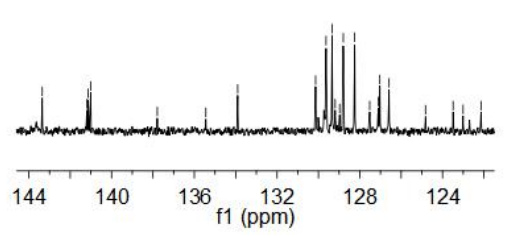

$\begin{array}{lllllllllllllllllllllll}210 & 200 & 190 & 180 & 170 & 160 & 150 & 140 & 130 & 120 & 110 & 100 & 90 & 80 & 70 & 60 & 50 & 40 & 30 & 20 & 10 & 0 & -10\end{array}$ 
Figure S11. The ${ }^{1} \mathrm{H}$ NMR spectrums of [Pd(IPrOMe* IMe $\left.)^{\mathrm{An}}(3-\mathrm{Cl}-\mathrm{pyridinyl}) \mathrm{Cl}_{2}\right](\mathrm{C2})$

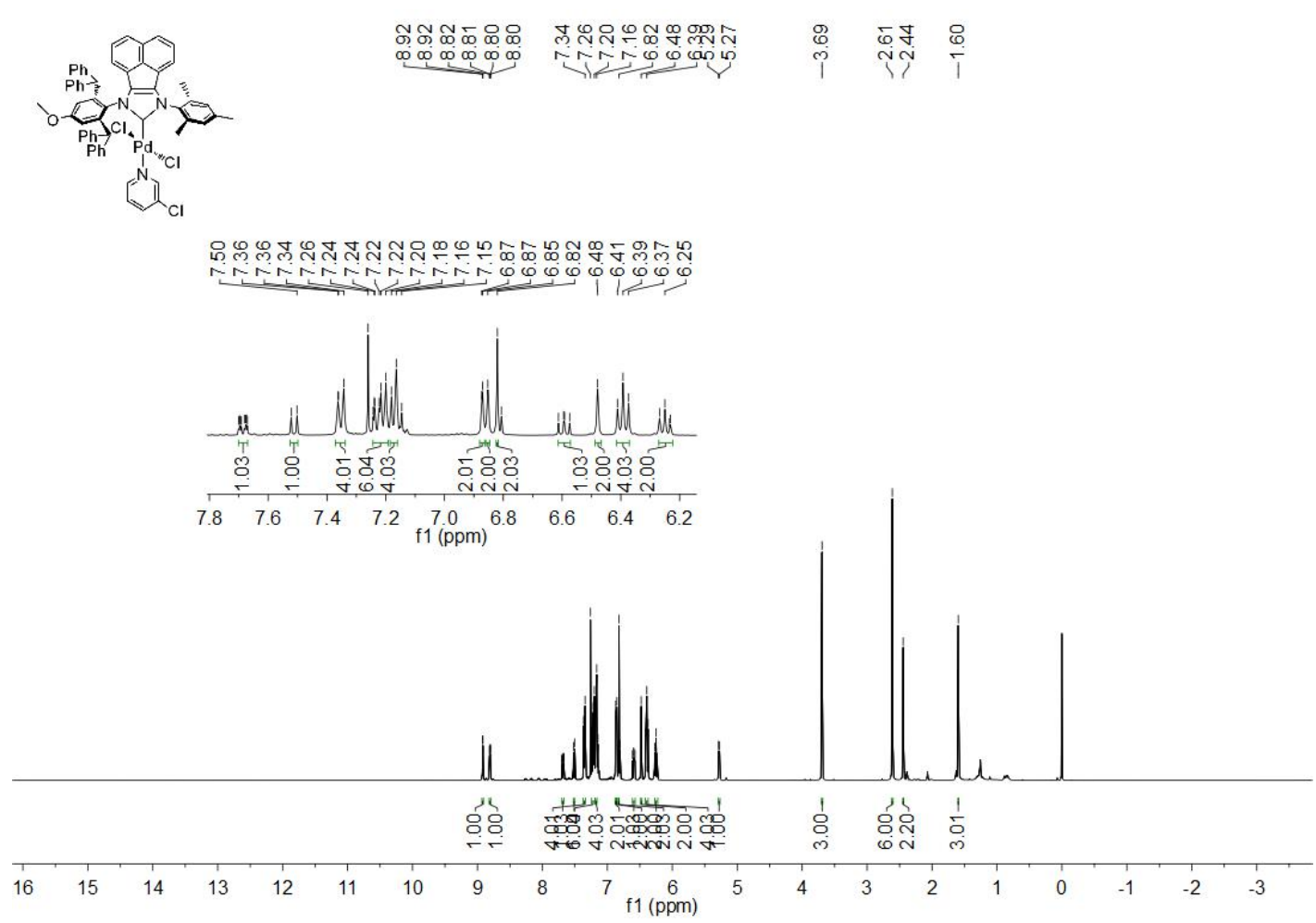

Figure S12. The ${ }^{13} \mathrm{C}$ NMR spectrums of $\left[\mathrm{Pd}\left(\mathrm{IPr}^{\mathrm{OMe} *} \mathrm{IMe}\right)^{\mathrm{An}}(3-\mathrm{Cl}-\mathrm{pyridinyl}) \mathrm{Cl}_{2}\right](\mathrm{C} 2)$

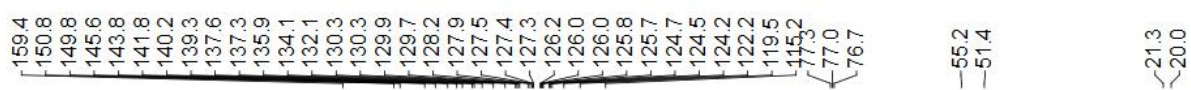
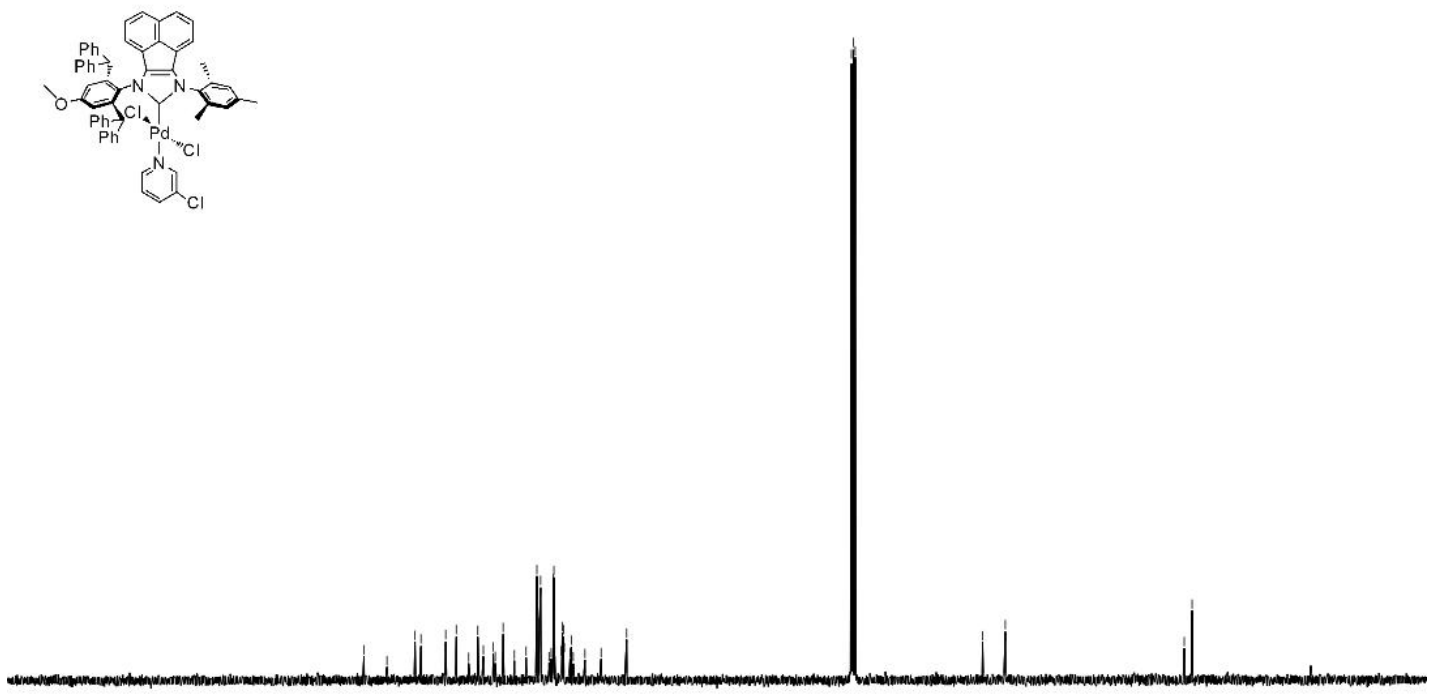

$\begin{array}{lllllllllllllllllllllll}210 & 200 & 190 & 180 & 170 & 160 & 150 & 140 & 130 & 120 & 110 & \begin{array}{c}100 \\ \mathrm{f} 1(\mathrm{ppm})\end{array} & 90 & 80 & 70 & 60 & 50 & 40 & 30 & 20 & 10 & 0 & -10\end{array}$ 
NMR spectrums of the arylated cross-coupling products

Figure S13. The ${ }^{1}$ H NMR spectrums of 2-methyl-5-(4-nitrophenyl)furan (3aa)
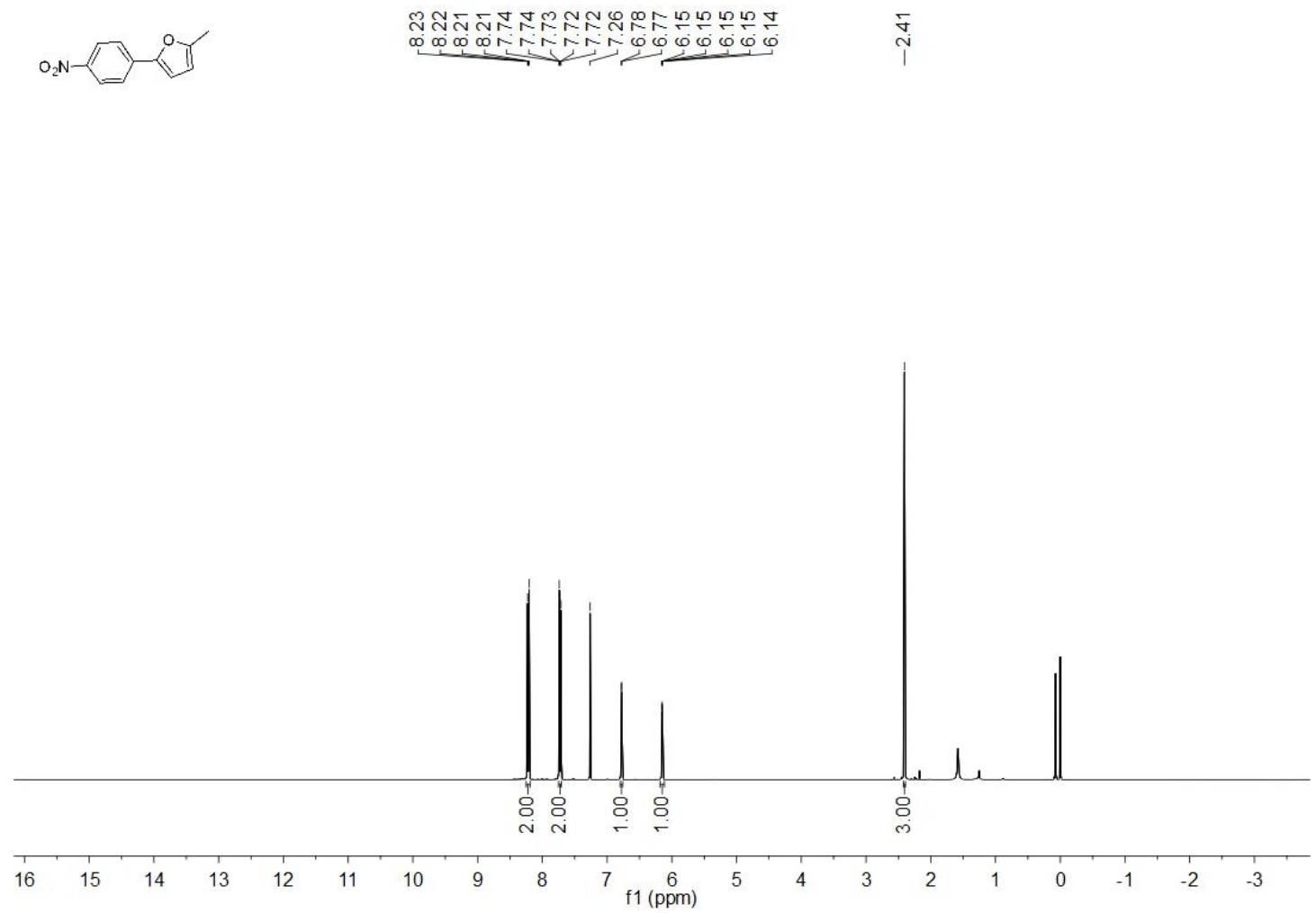

Figure S14. The ${ }^{13} \mathrm{C}$ NMR spectrums of 2-methyl-5-(4-nitrophenyl)furan (3aa)

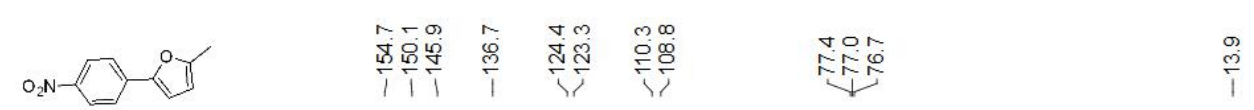

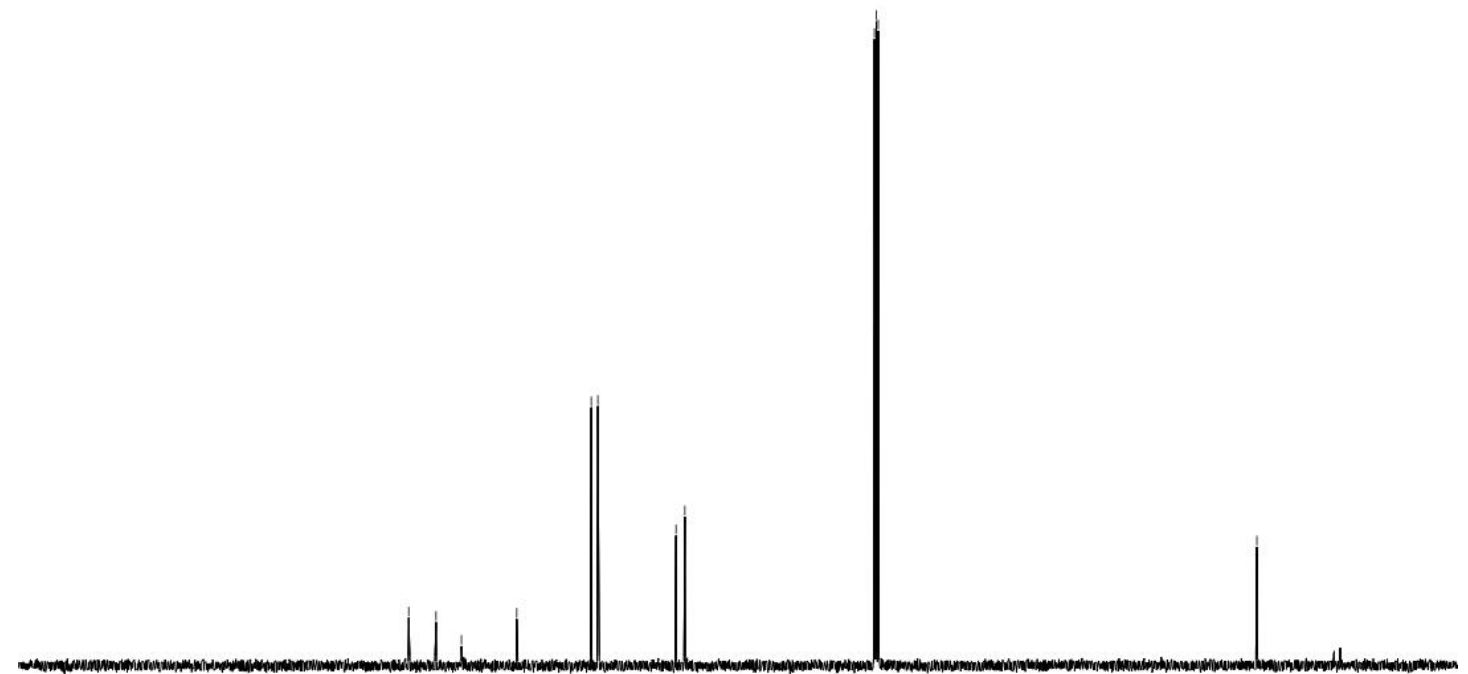

$\begin{array}{lllllllllllllllllllllll}210 & 200 & 190 & 180 & 170 & 160 & 150 & 140 & 130 & 120 & 110 & 100 & 90 & 80 & 70 & 60 & 50 & 40 & 30 & 20 & 10 & 0 & -10\end{array}$ 
Figure S15. The ${ }^{1} \mathrm{H}$ NMR spectrums of 4-(5-methylfuran-2-yl)benzonitrile (3ab)
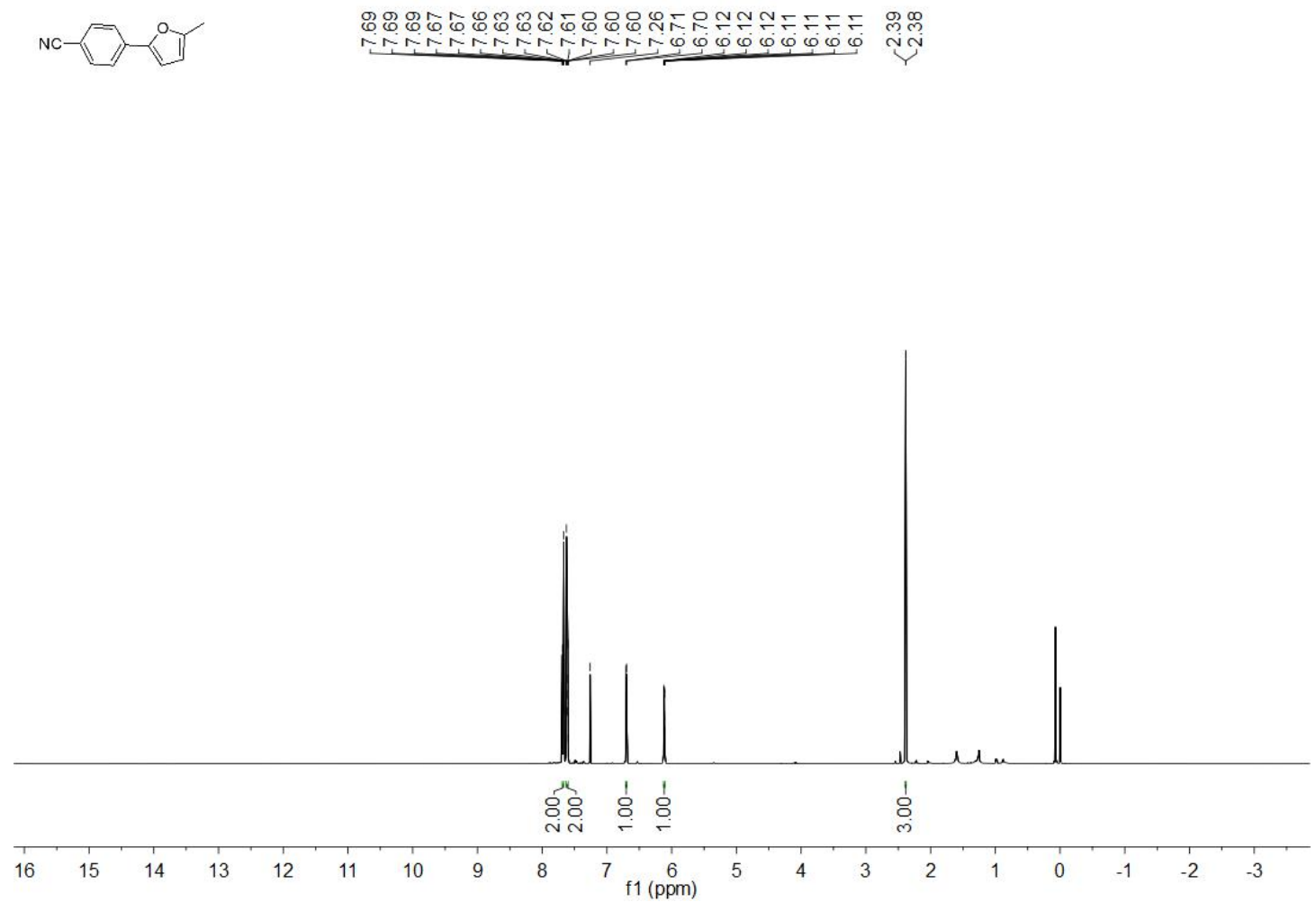

Figure S16. The ${ }^{13} \mathrm{C}$ NMR spectrums of 4-(5-methylfuran-2-yl)benzonitrile (3ab)

《1

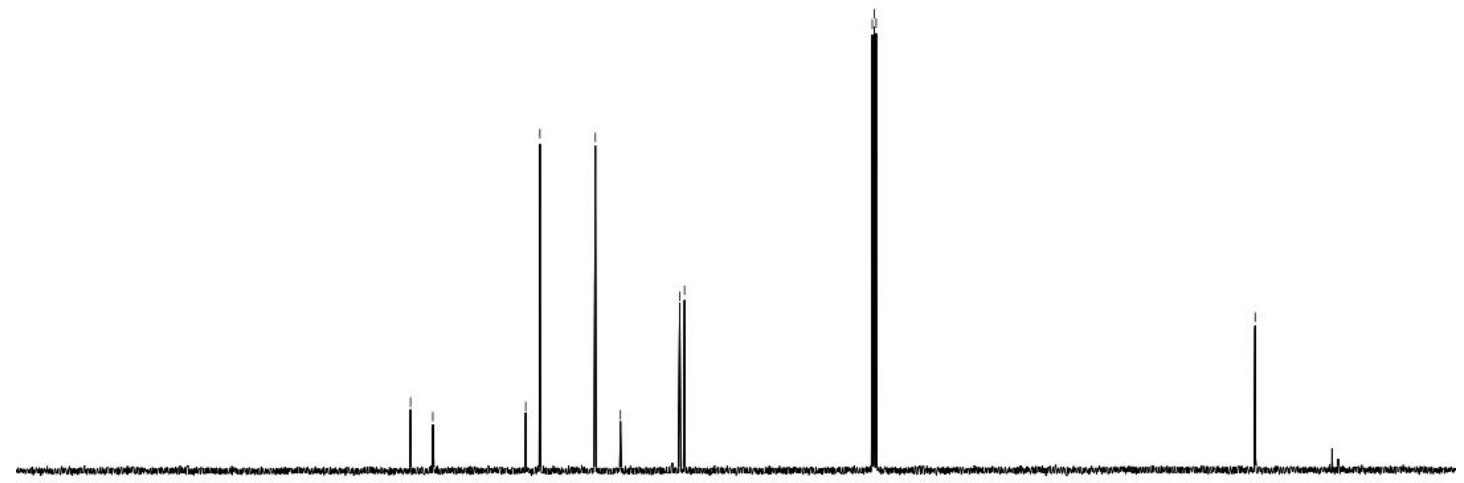

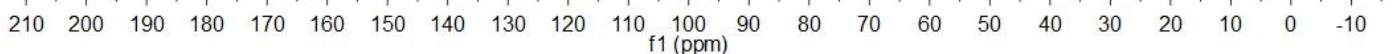


Figure S17. The ${ }^{1} \mathrm{H}$ NMR spectrums of 4-(5-methylfuran-2-yl)benzaldehyde (3ac)
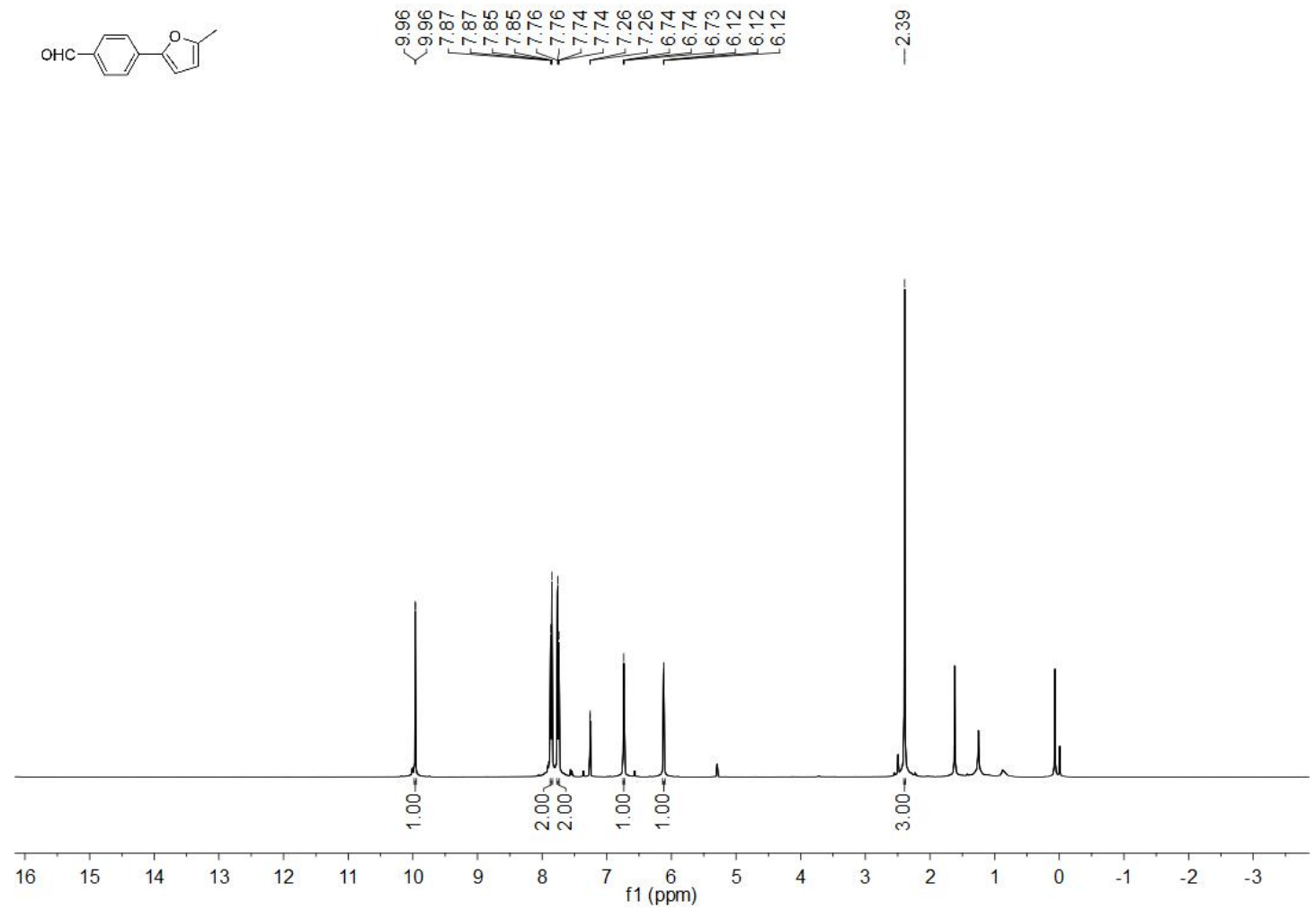

Figure S18. The ${ }^{13} \mathrm{C}$ NMR spectrums of 4-(5-methylfuran-2-yl)benzaldehyde (3ac)

蒠
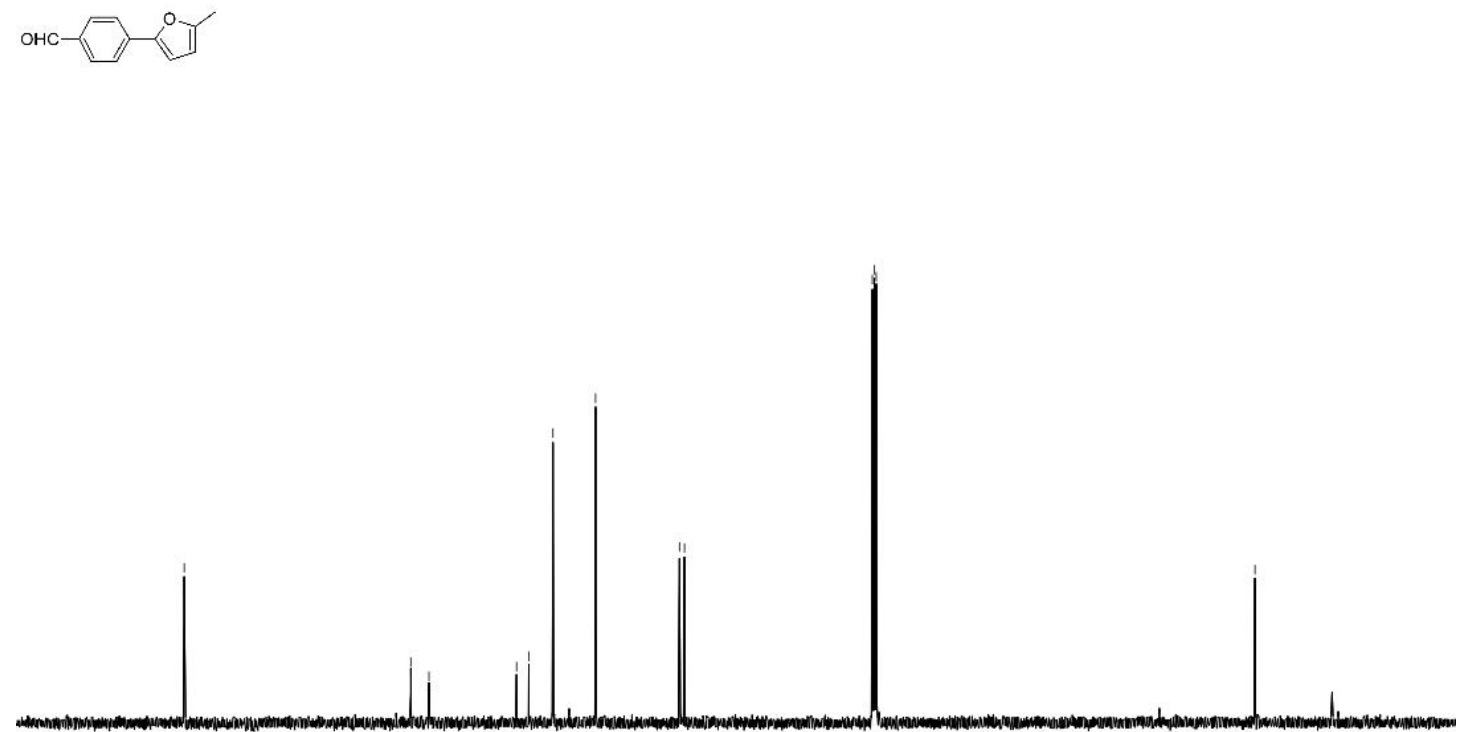

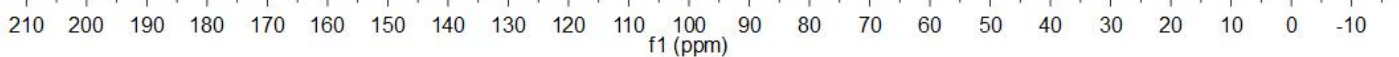


Figure S19. The ${ }^{1}$ H NMR spectrums of 1-(4-(5-methylfuran-2-yl)phenyl)ethanone (3ad)

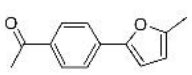

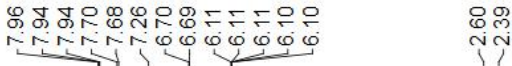

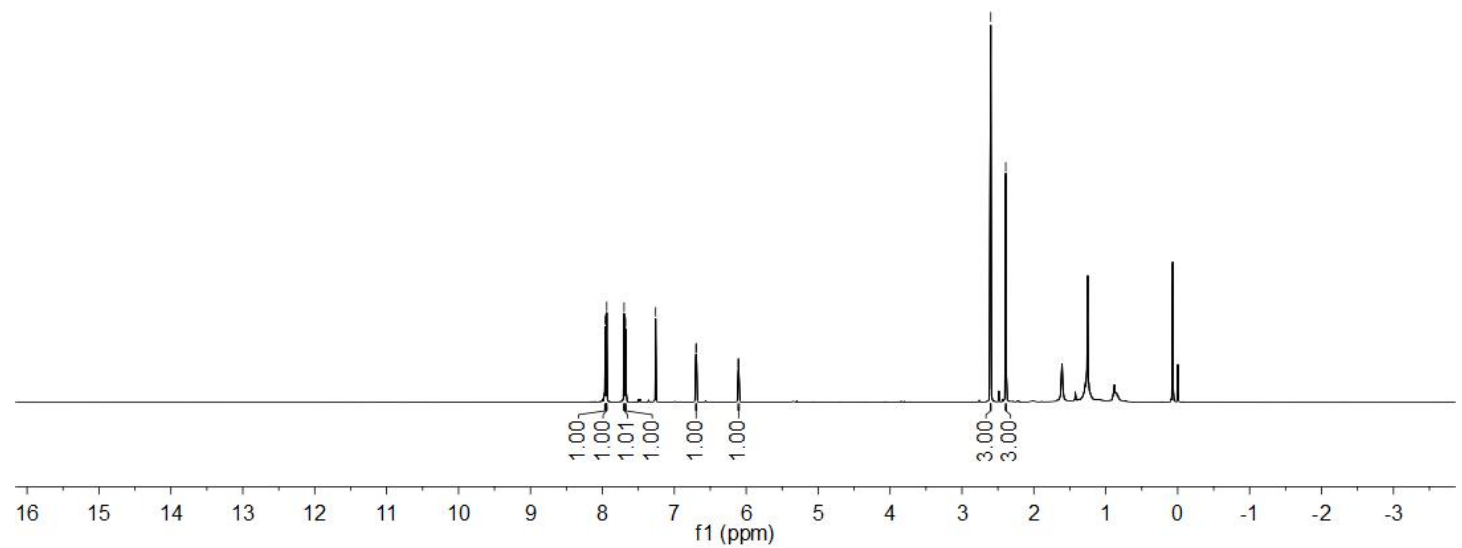

Figure S20. The ${ }^{13} \mathrm{C}$ NMR spectrums of 1-(4-(5-methylfuran-2-yl)phenyl)ethanone (3ad)

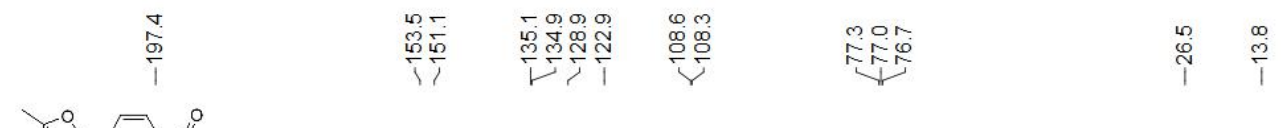

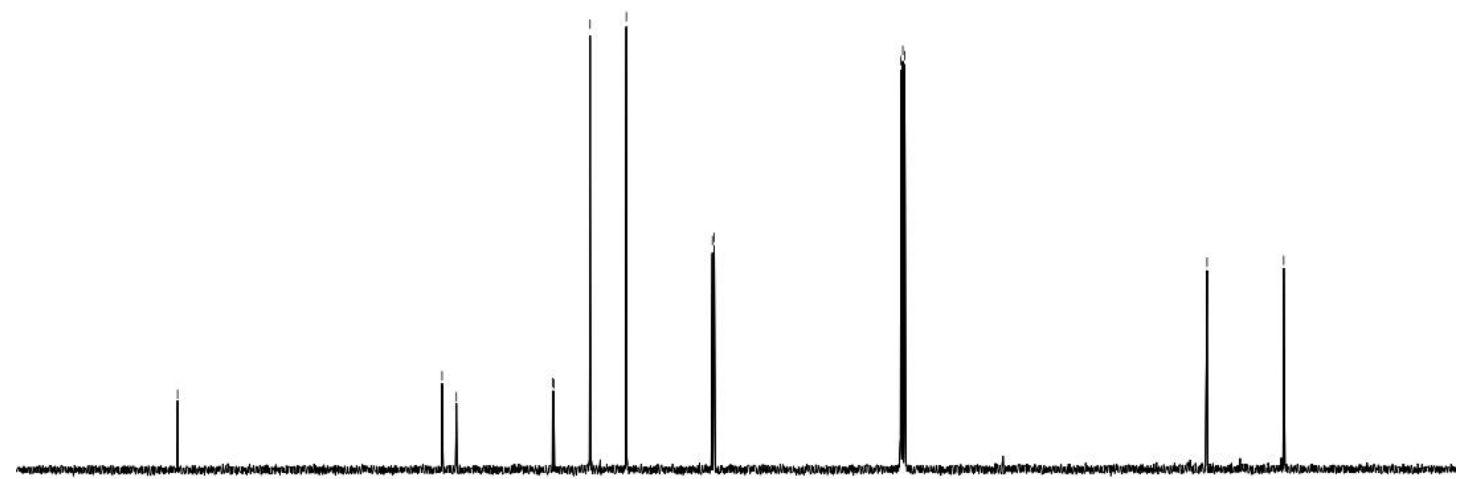

$\begin{array}{lllllllllllllllllllllll}220 & 210 & 200 & 190 & 180 & 170 & 160 & 150 & 140 & 130 & 120 & \begin{array}{c}110 \\ \mathrm{f} 1(\mathrm{ppm})\end{array} & 90 & 80 & 70 & 60 & 50 & 40 & 30 & 20 & 10 & 0 & -10\end{array}$ 
Figure S21. The ${ }^{1} \mathrm{H}$ NMR spectrums of 2-(4-chlorophenyl)-5-methylfuran (3ae)
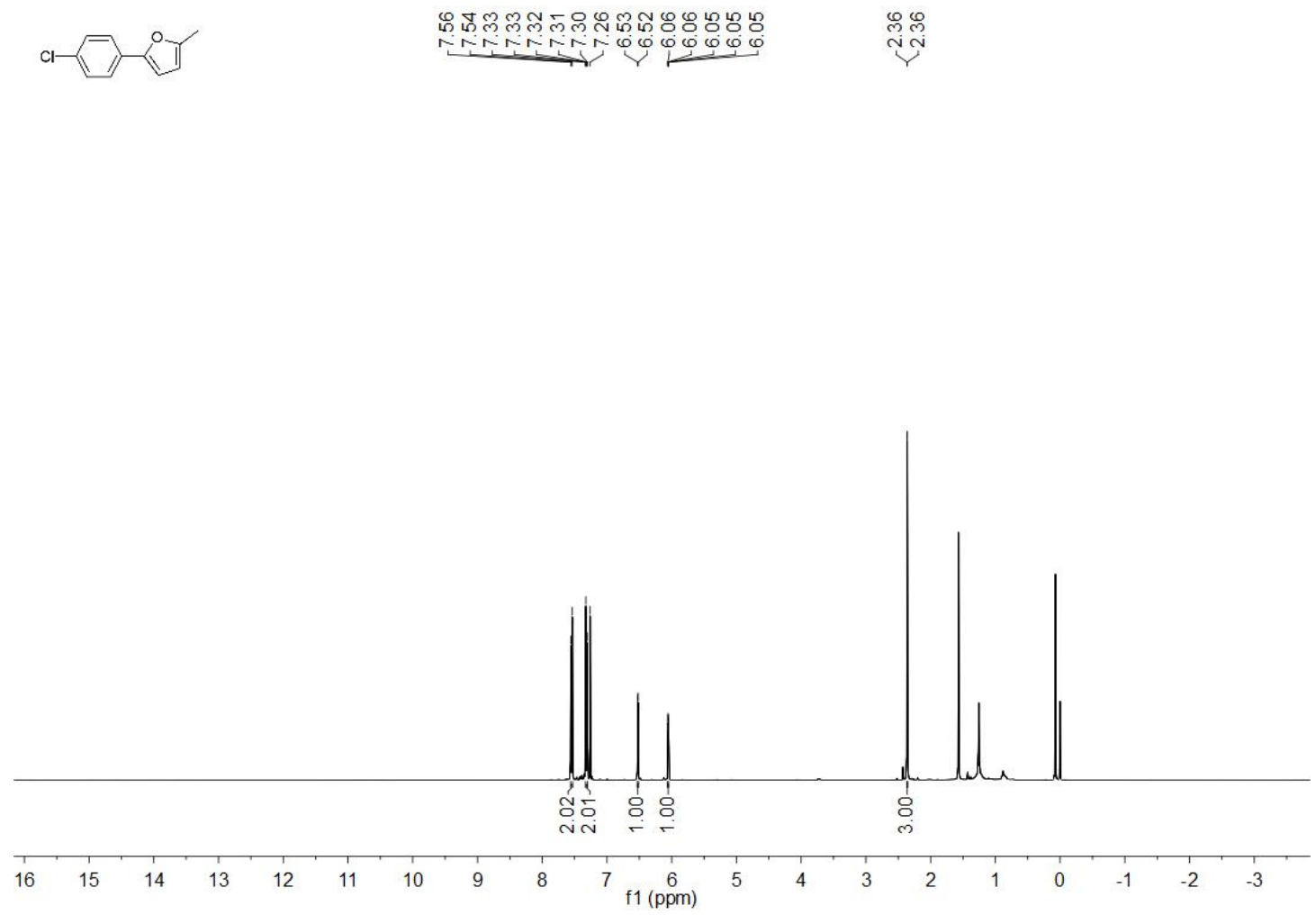

Figure S22. The ${ }^{13}$ C NMR spectrums of 2-(4-chlorophenyl)-5-methylfuran (3ae)

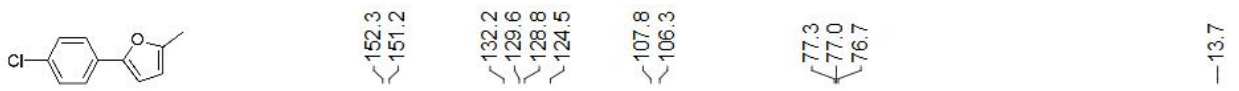

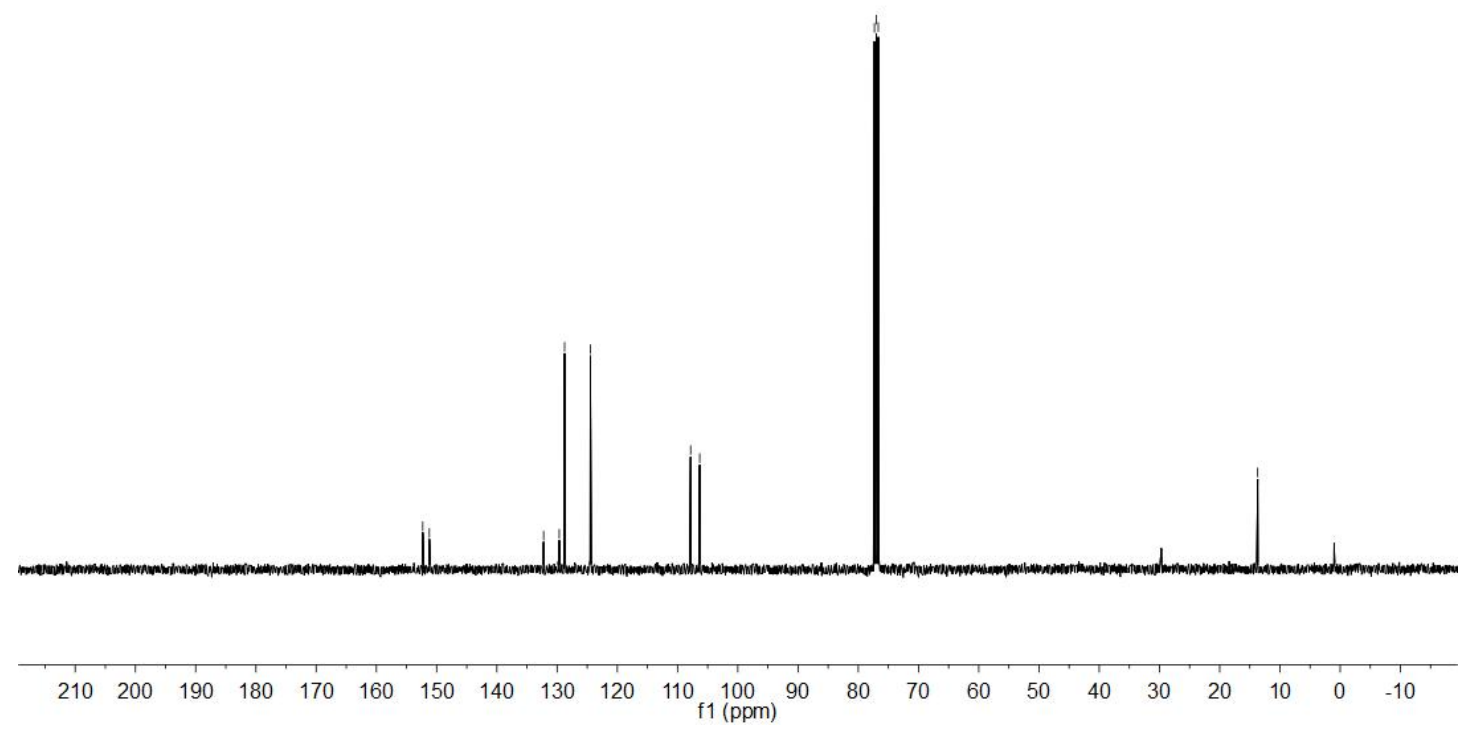


Figure S23. The ${ }^{1} \mathrm{H}$ NMR spectrums of 2-methyl-5-phenylfuran (3af)

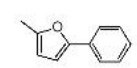

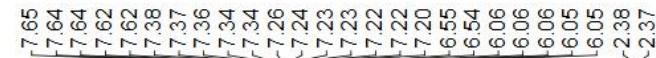

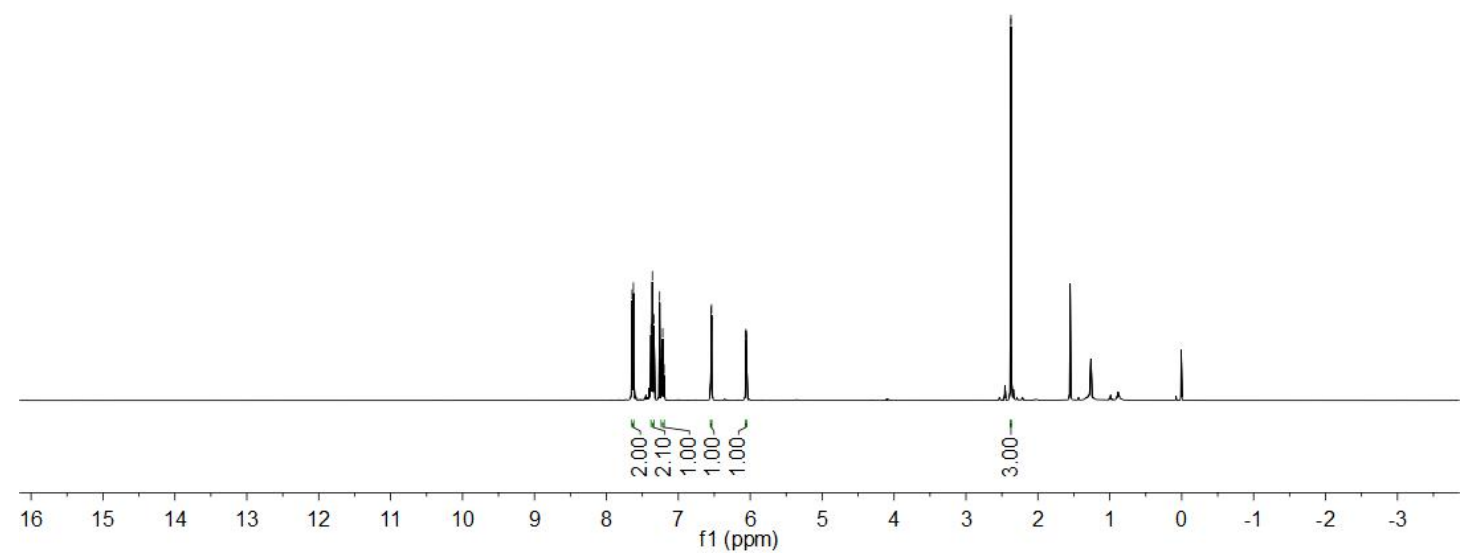

Figure S24. The ${ }^{13} \mathrm{C}$ NMR spectrums of 2-methyl-5-phenylfuran (3af)

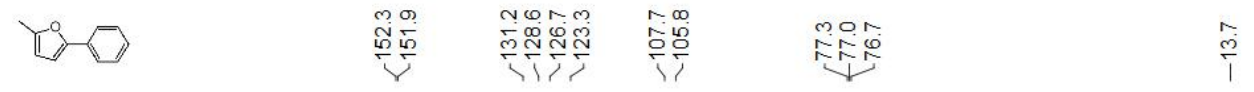

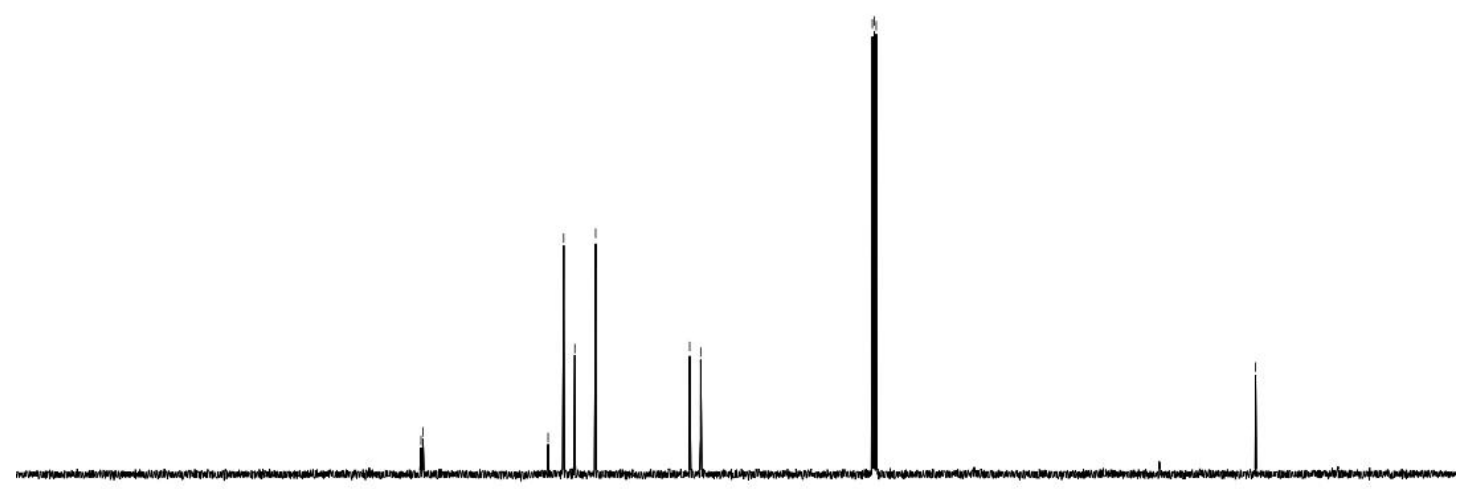

$\begin{array}{lllllllllllllllllllllll}210 & 200 & 190 & 180 & 170 & 160 & 150 & 140 & 130 & 120 & 110 \begin{array}{c}100 \\ \mathrm{f} 1(\mathrm{ppm})\end{array} & 90 & 80 & 70 & 60 & 50 & 40 & 30 & 20 & 10 & 0 & -10\end{array}$ 
Figure S25. The ${ }^{1} \mathrm{H}$ NMR spectrums of 2-(4-bromophenyl)-5-methylfuran (3ag)

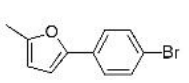

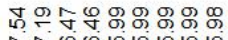

T.

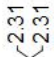

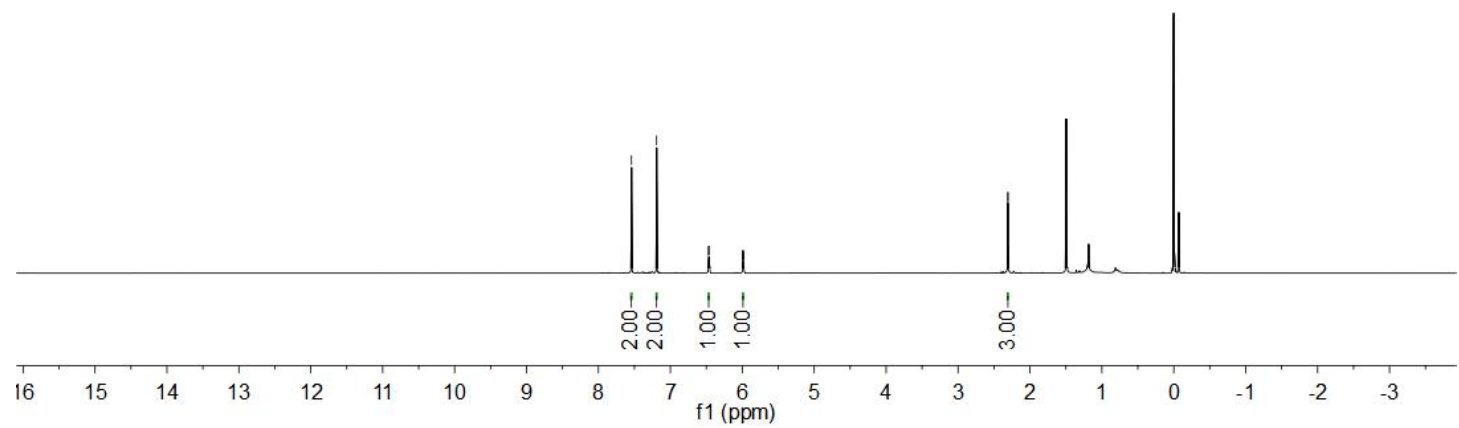

Figure S26. The ${ }^{13} \mathrm{C}$ NMR spectrums of 2-(4-bromophenyl)-5-methylfuran (3ag)

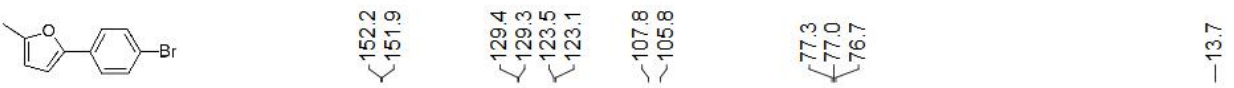

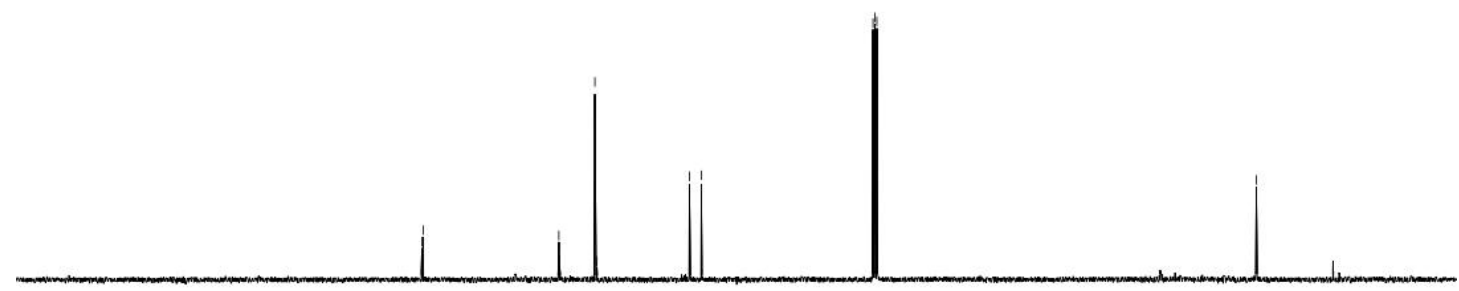

$\begin{array}{llllllllllllllllllllll}210 & 200 & 190 & 180 & 170 & 160 & 150 & 140 & 130 & 120 & 110 \begin{array}{l}100 \\ \mathrm{f} 1(\mathrm{ppm})\end{array} & 90 & 80 & 70 & 60 & 50 & 40 & 30 & 20 & 10 & 0 & -10\end{array}$ 
Figure S27. The ${ }^{1} \mathrm{H}$ NMR spectrums of 2-fluoro-5-(5-methylfuran-2-yl)pyridine (3ah)
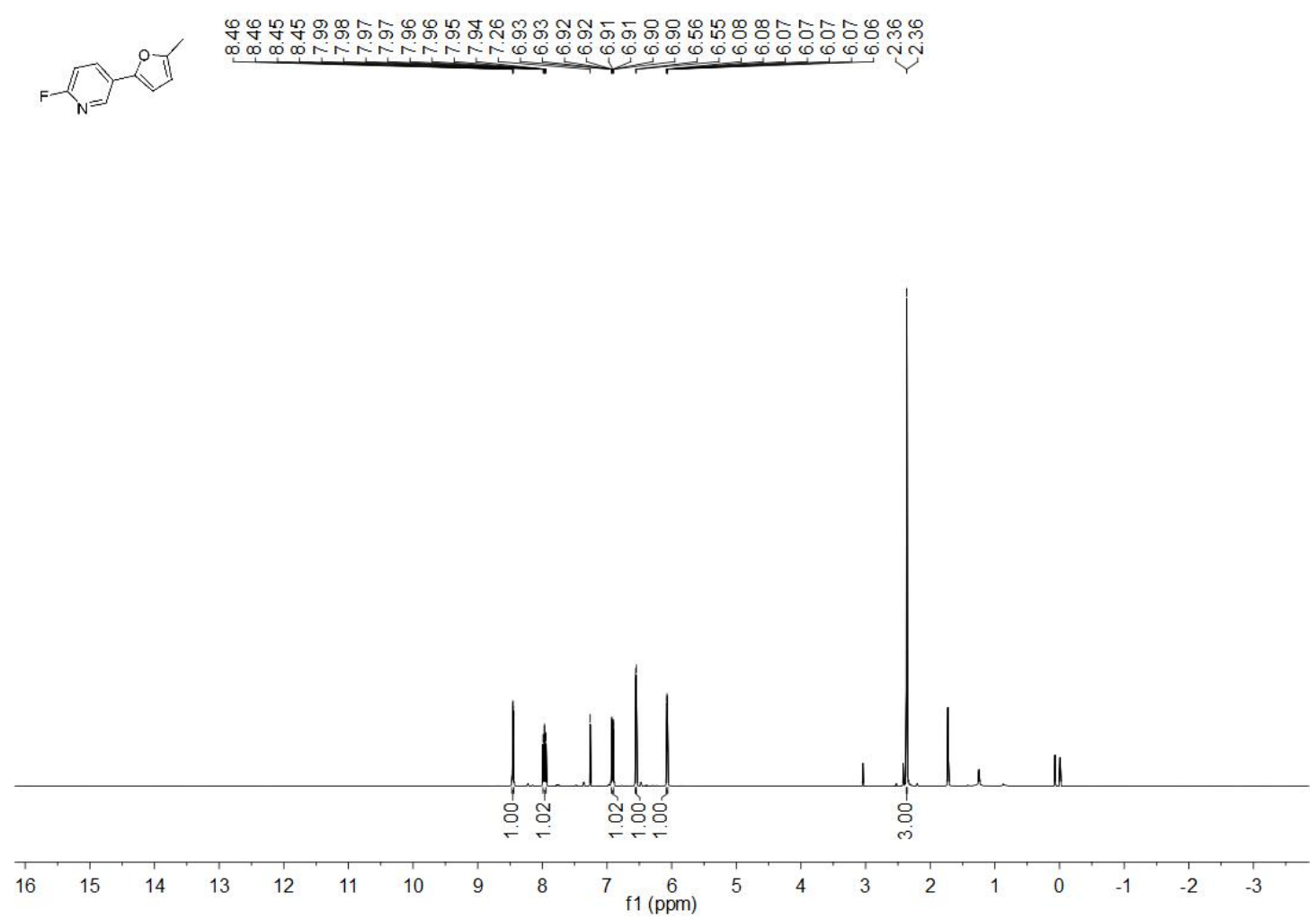

Figure S28. The ${ }^{13} \mathrm{C}$ NMR spectrums of 2-fluoro-5-(5-methylfuran-2-yl)pyridine (3ah)
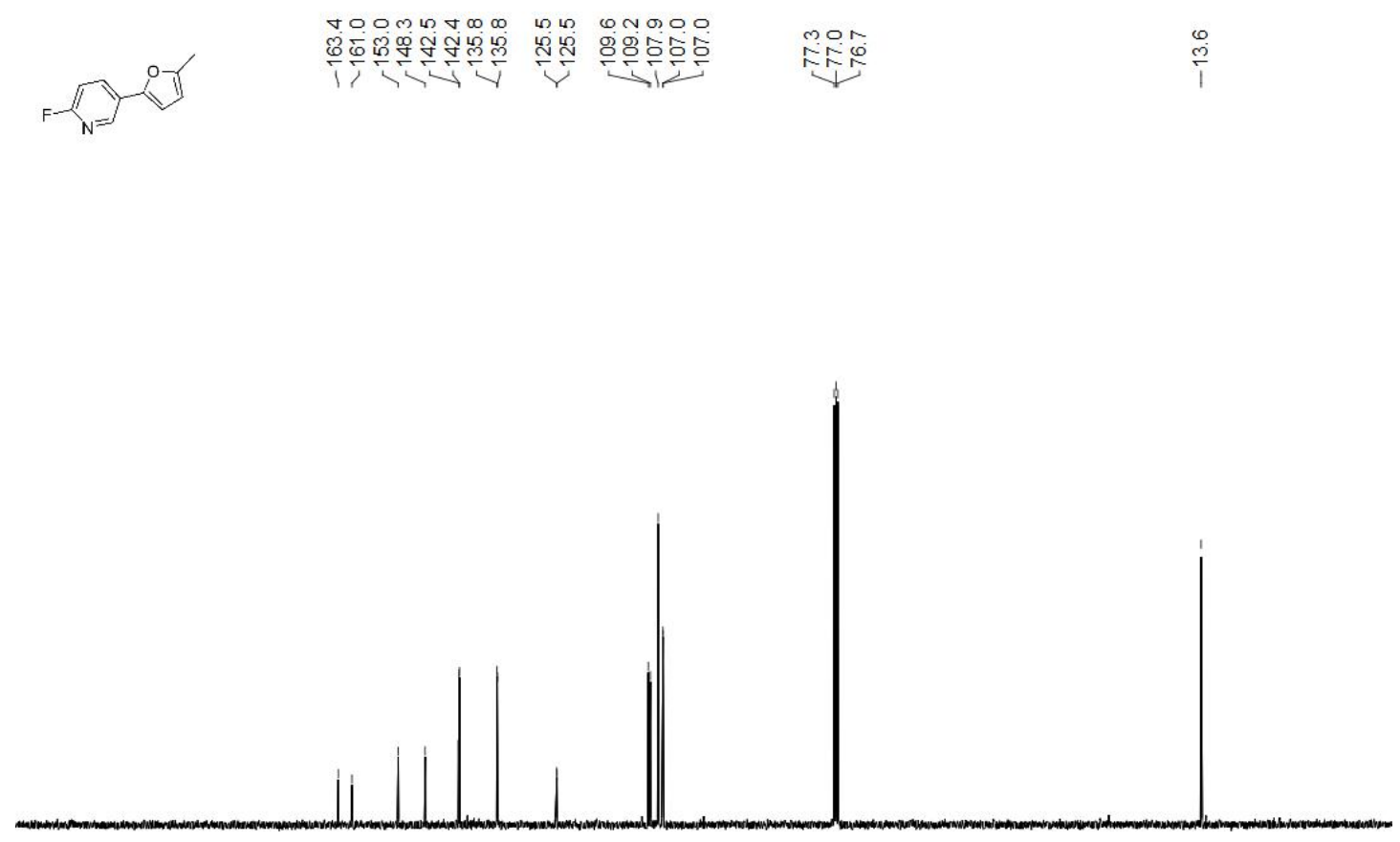

$\begin{array}{llllllllllllllllllllll}210 & 200 & 190 & 180 & 170 & 160 & 150 & 140 & 130 & 120 & 110 \begin{array}{c}100 \\ \mathrm{f} 1(\mathrm{ppm})\end{array} & 90 & 80 & 70 & 60 & 50 & 40 & 30 & 20 & 10 & 0 & -10\end{array}$ 
Figure S29. The ${ }^{1}$ H NMR spectrums of 2-methyl-5-(5-methylfuran-2-yl)pyridine (3ai)
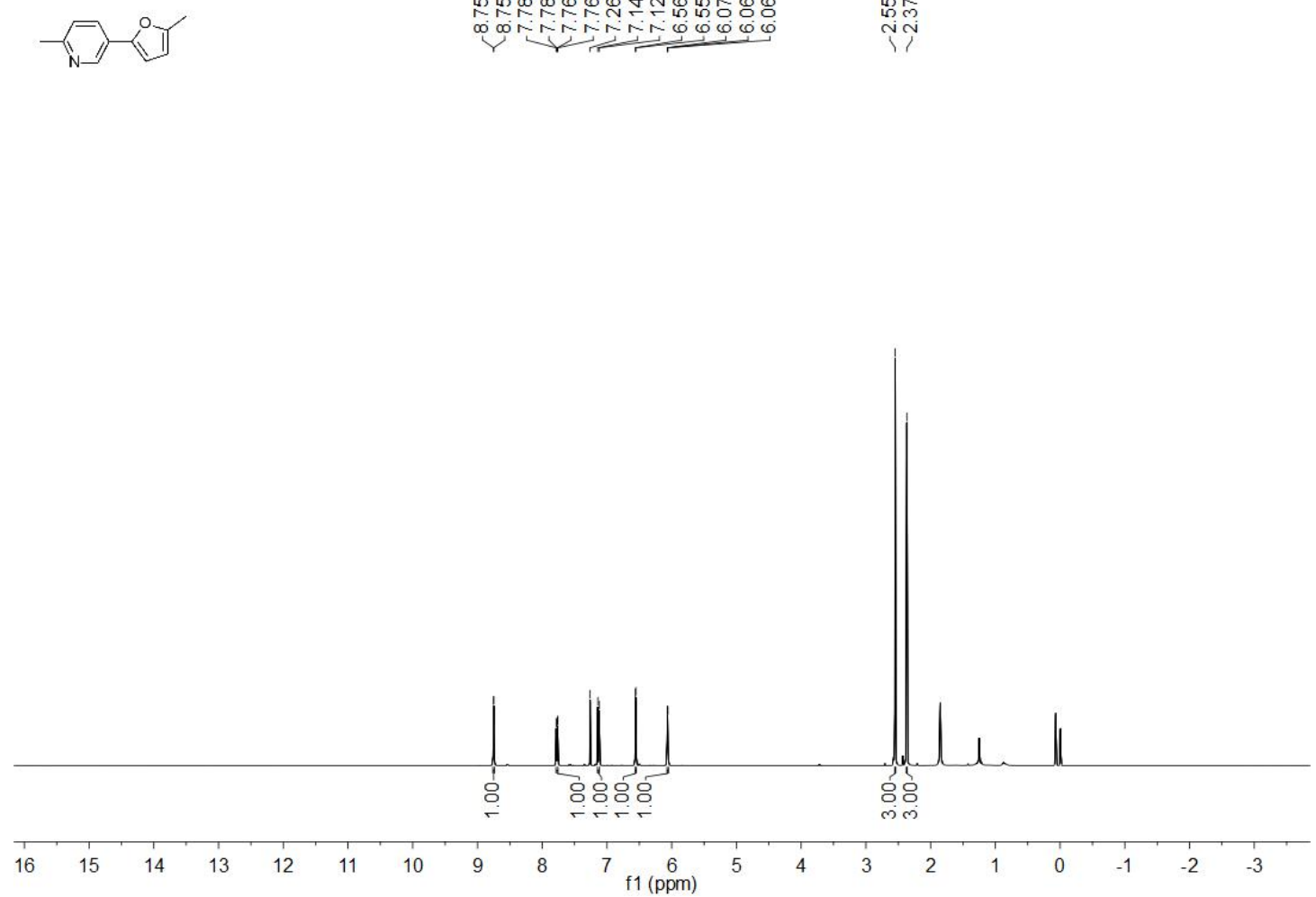

Figure S30. The ${ }^{13} \mathrm{C}$ NMR spectrums of 2-methyl-5-(5-methylfuran-2-yl)pyridine (3ai)

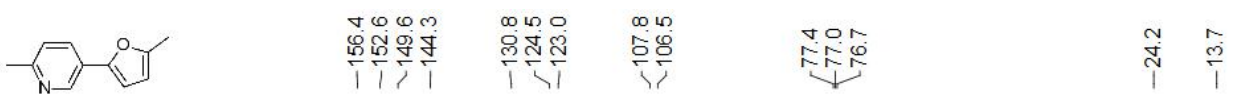

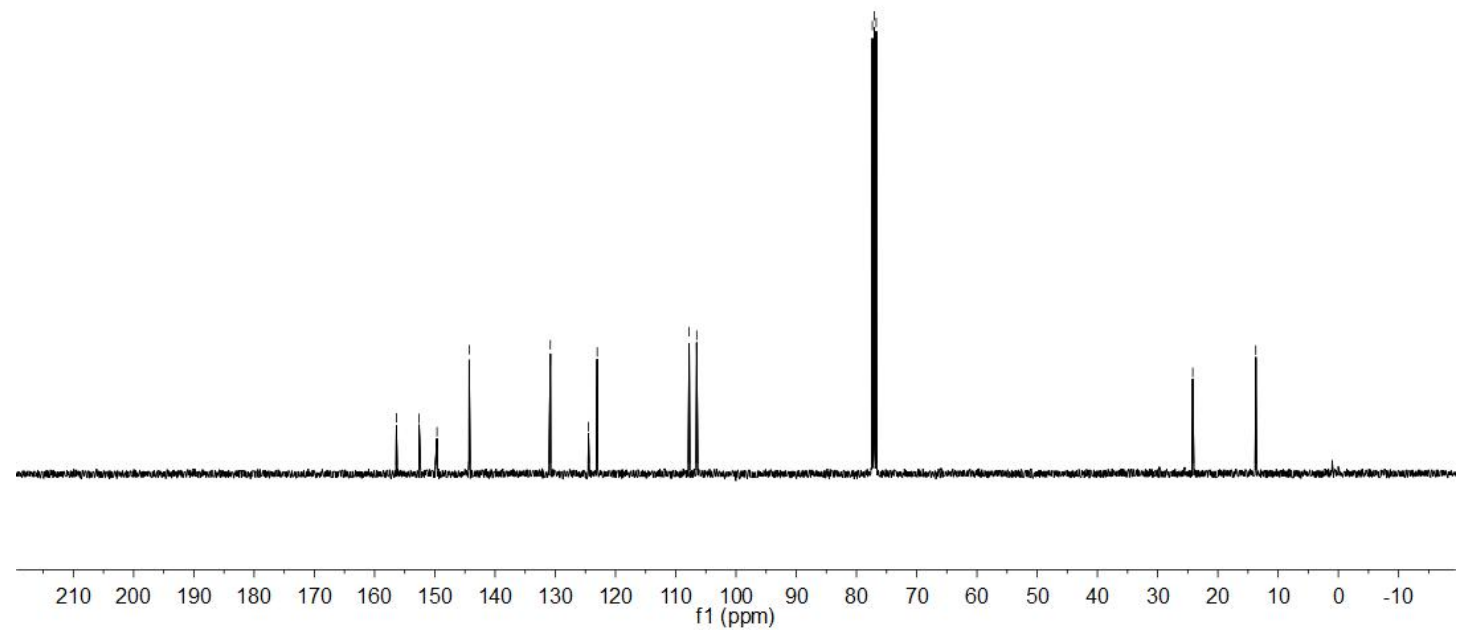


Figure S31. The ${ }^{1} \mathrm{H}$ NMR spectrums of 3-(5-methylfuran-2-yl)pyridine (3aj)
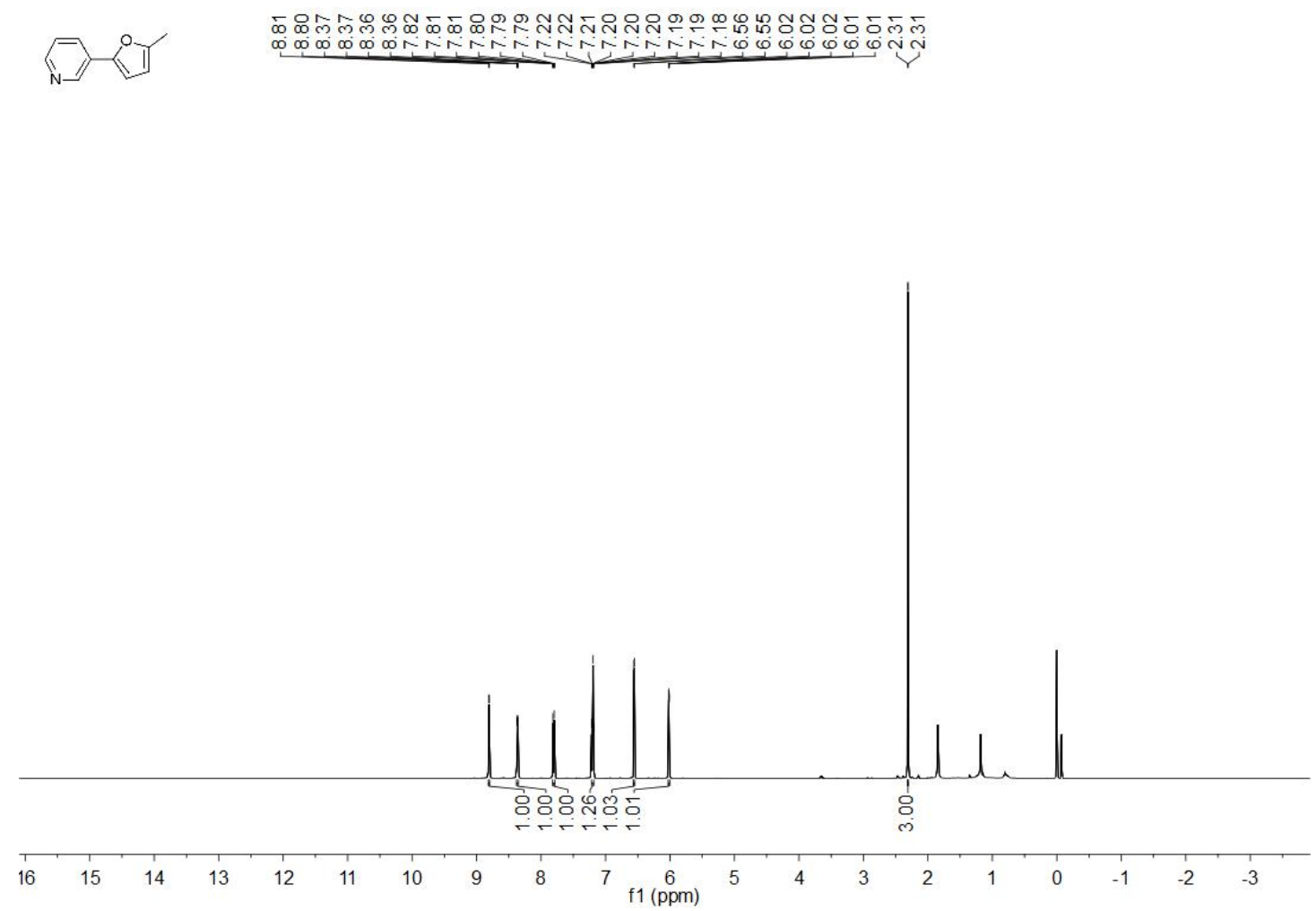

Figure S32. The ${ }^{13} \mathrm{C}$ NMR spectrums of 3-(5-methylfuran-2-yl)pyridine (3aj)

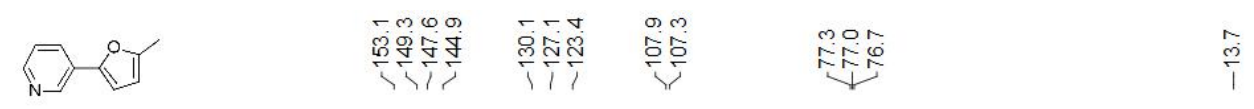

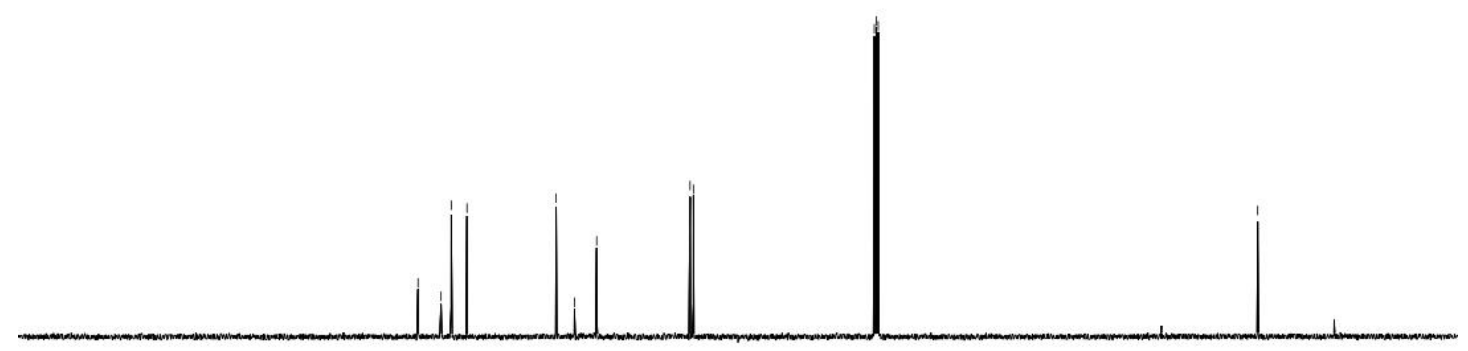

$\begin{array}{llllllllllllllllllllllll}210 & 200 & 190 & 180 & 170 & 160 & 150 & 140 & 130 & 120 & 110 & 100 & 90 & 80 & 70 & 60 & 50 & 40 & 30 & 20 & 10 & 0 & -10\end{array}$ 
Figure S33. The ${ }^{1} \mathrm{H}$ NMR spectrums of 4-(5-methylfuran-2-yl)isoquinoline (3ak)

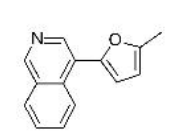

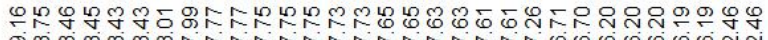

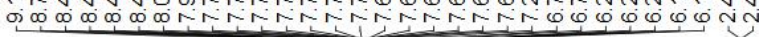

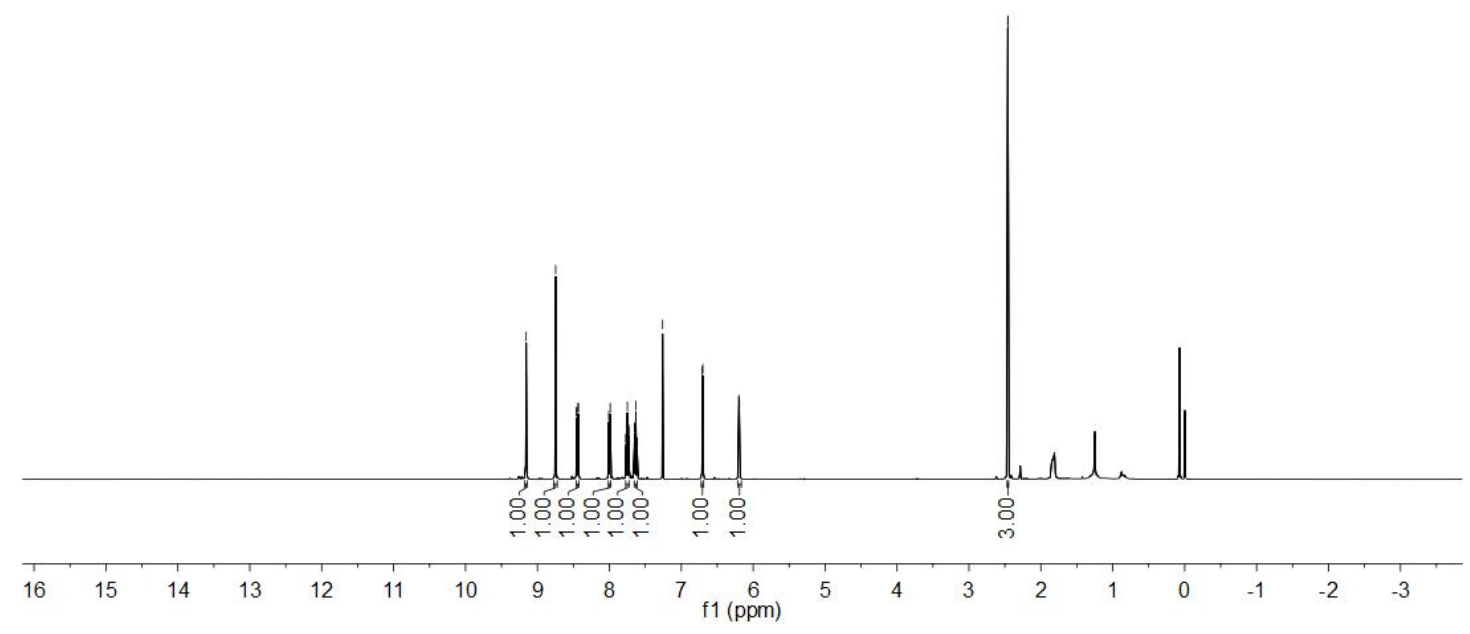

Figure S34. The ${ }^{13} \mathrm{C}$ NMR spectrums of 4-(5-methylfuran-2-yl)isoquinoline (3ak)
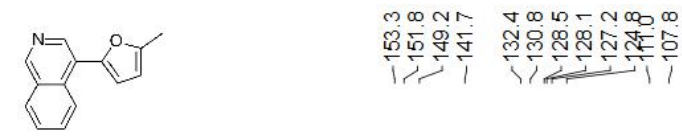

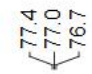

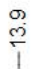

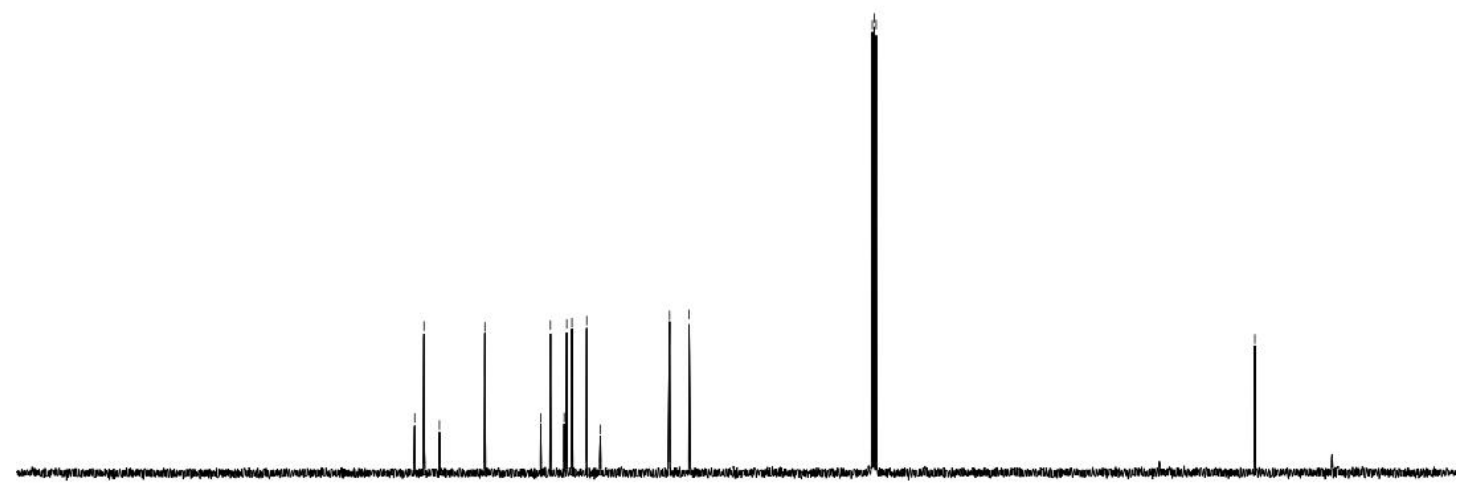

$\begin{array}{llllllllllllllllllllll}210 & 200 & 190 & 180 & 170 & 160 & 150 & 140 & 130 & 120 & 110 \begin{array}{l}100 \\ \mathrm{f} 1(\mathrm{ppm})\end{array} & 90 & 80 & 70 & 60 & 50 & 40 & 30 & 20 & 10 & 0 & -10\end{array}$ 
Figure S35. The ${ }^{1} \mathrm{H}$ NMR spectrums of 3-(5-methylfuran-2-yl)quinolone (3al)
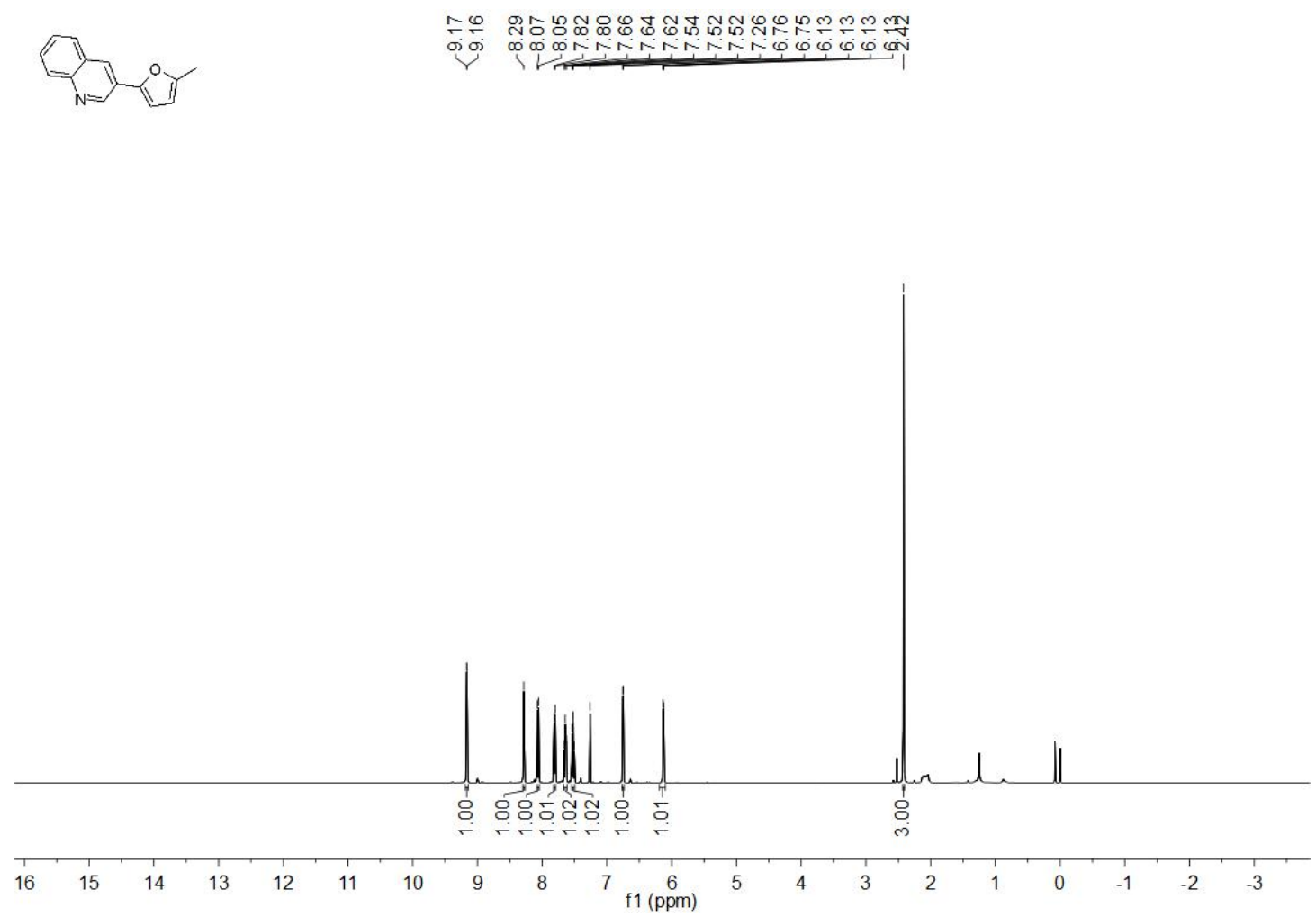

Figure S36. The ${ }^{13} \mathrm{C}$ NMR spectrums of 3-(5-methylfuran-2-yl)quinolone (3al)
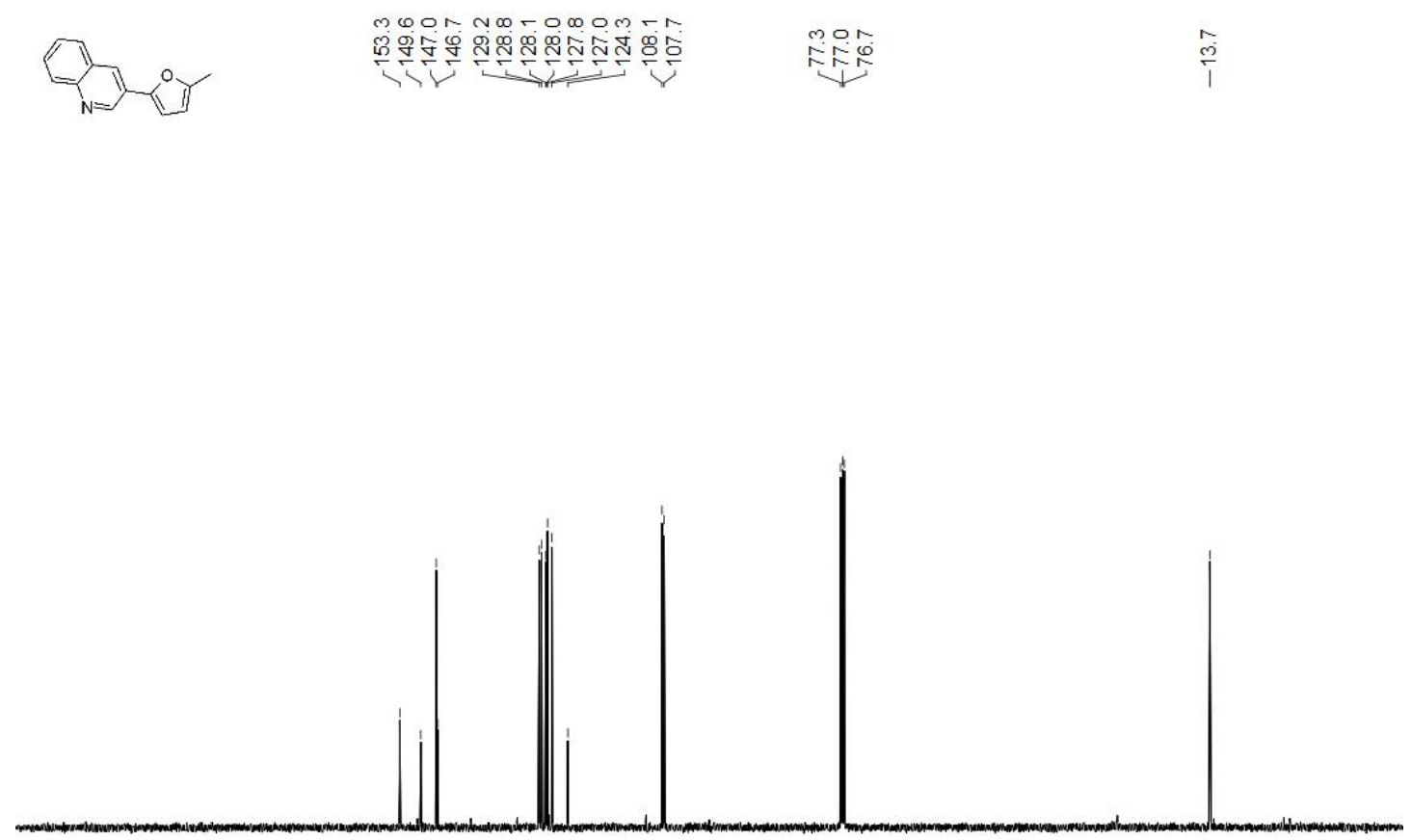

$\begin{array}{llllllllllllllllllllll}210 & 200 & 190 & 180 & 170 & 160 & 150 & 140 & 130 & 120 & 110 \begin{array}{c}100 \\ \mathrm{f} 1(\mathrm{ppm})\end{array} & 90 & 80 & 70 & 60 & 50 & 40 & 30 & 20 & 10 & 0 & -10\end{array}$ 
Figure S37. The ${ }^{1} \mathrm{H}$ NMR spectrums of 2-methyl-5-(naphthalen-1-yl)furan (3am)
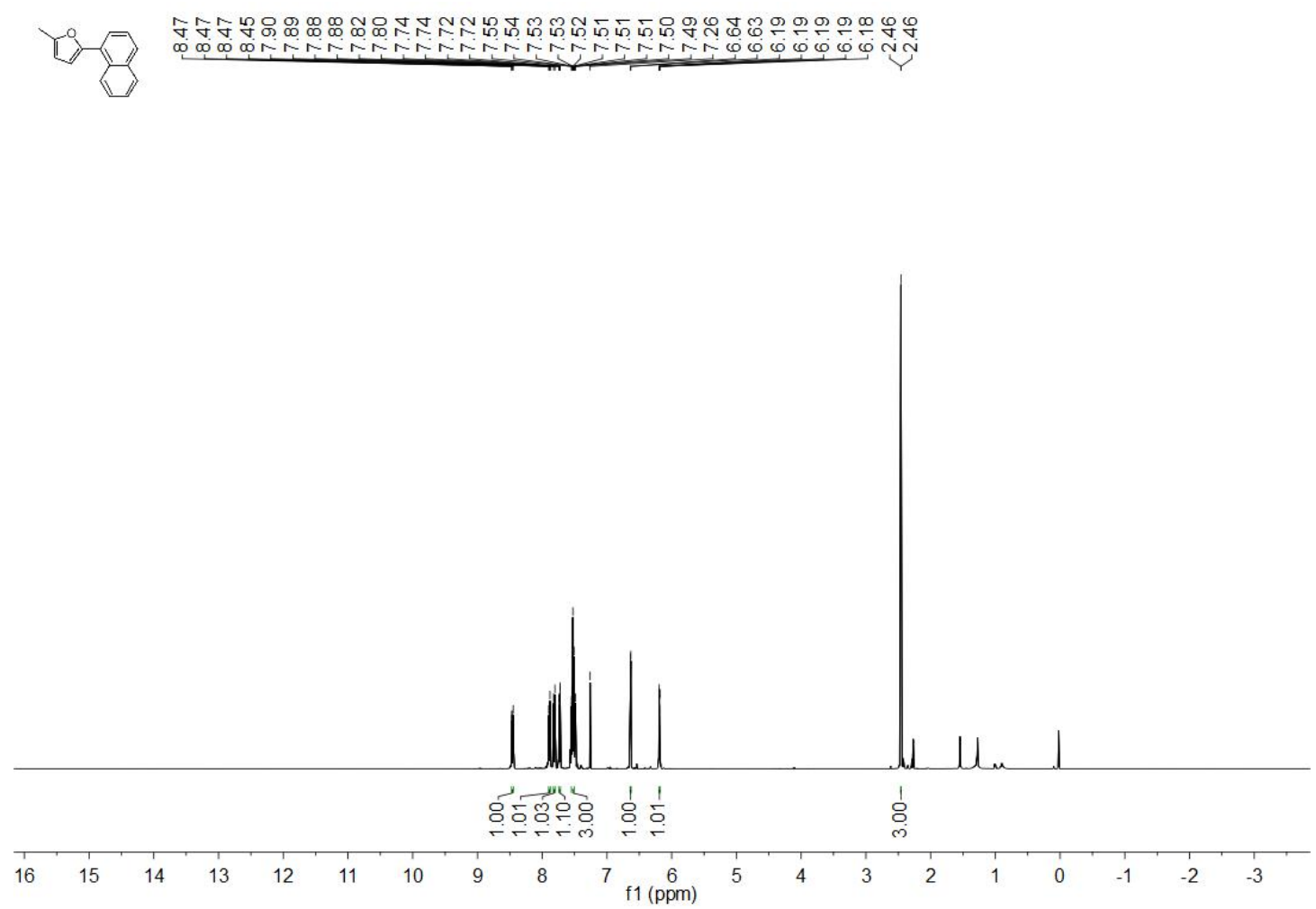

Figure S38. The ${ }^{13}$ C NMR spectrums of 2-methyl-5-(naphthalen-1-yl)furan (3am)
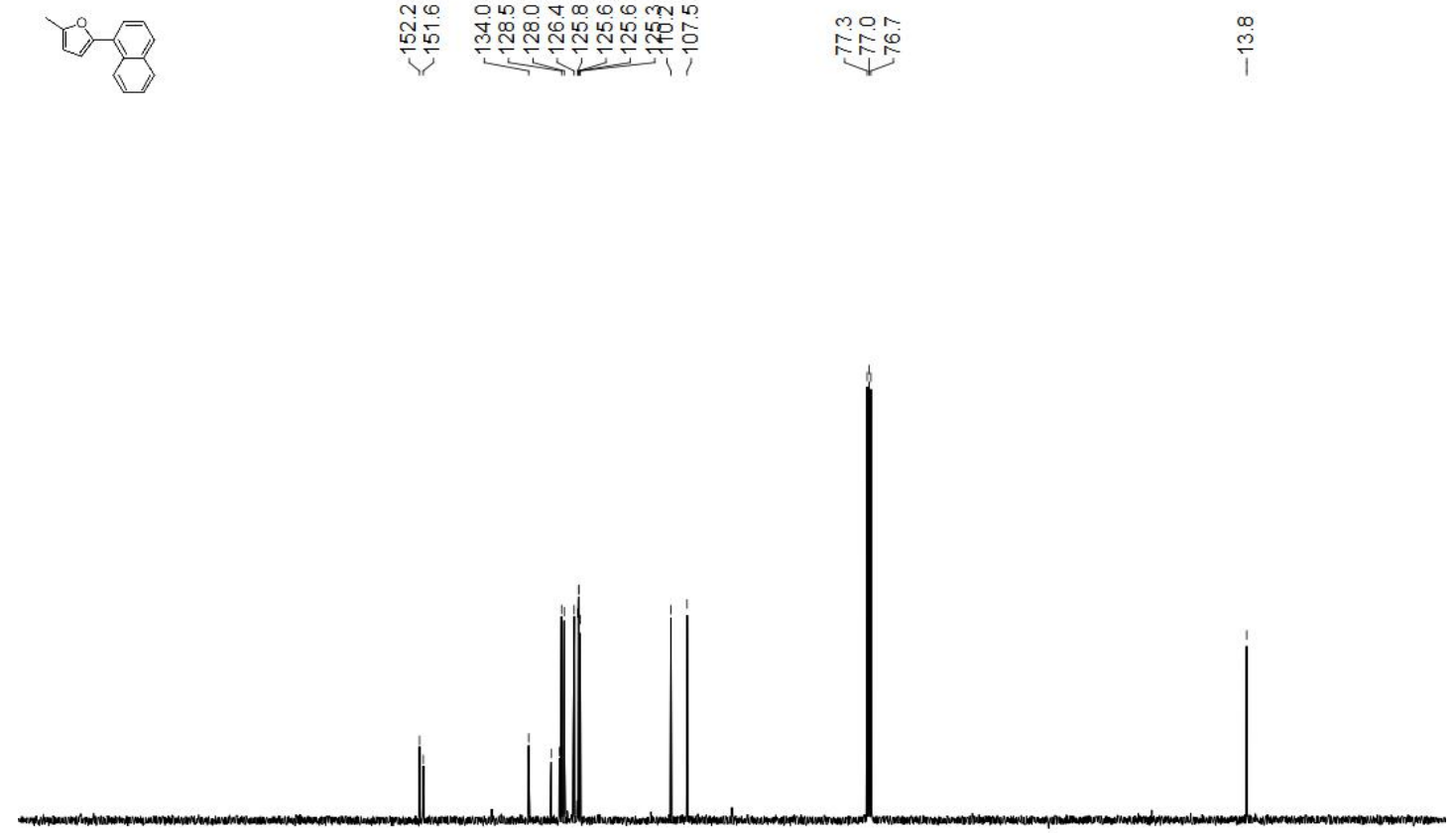

$\begin{array}{llllllllllllllllllllll}210 & 200 & 190 & 180 & 170 & 160 & 150 & 140 & 130 & 120 & 110 \begin{array}{l}100 \\ \mathrm{f} 1(\mathrm{ppm})\end{array} & 90 & 80 & 70 & 60 & 50 & 40 & 30 & 20 & 10 & 0 & -10\end{array}$ 
Figure S39. The ${ }^{1} \mathrm{H}$ NMR spectrums of 2-methyl-5-(2-methylnaphthalen-1-yl)furan (3an)

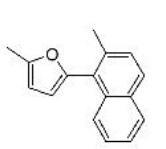

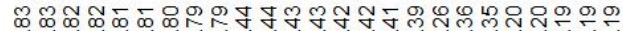

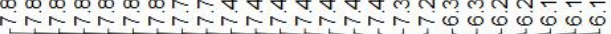

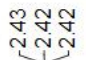

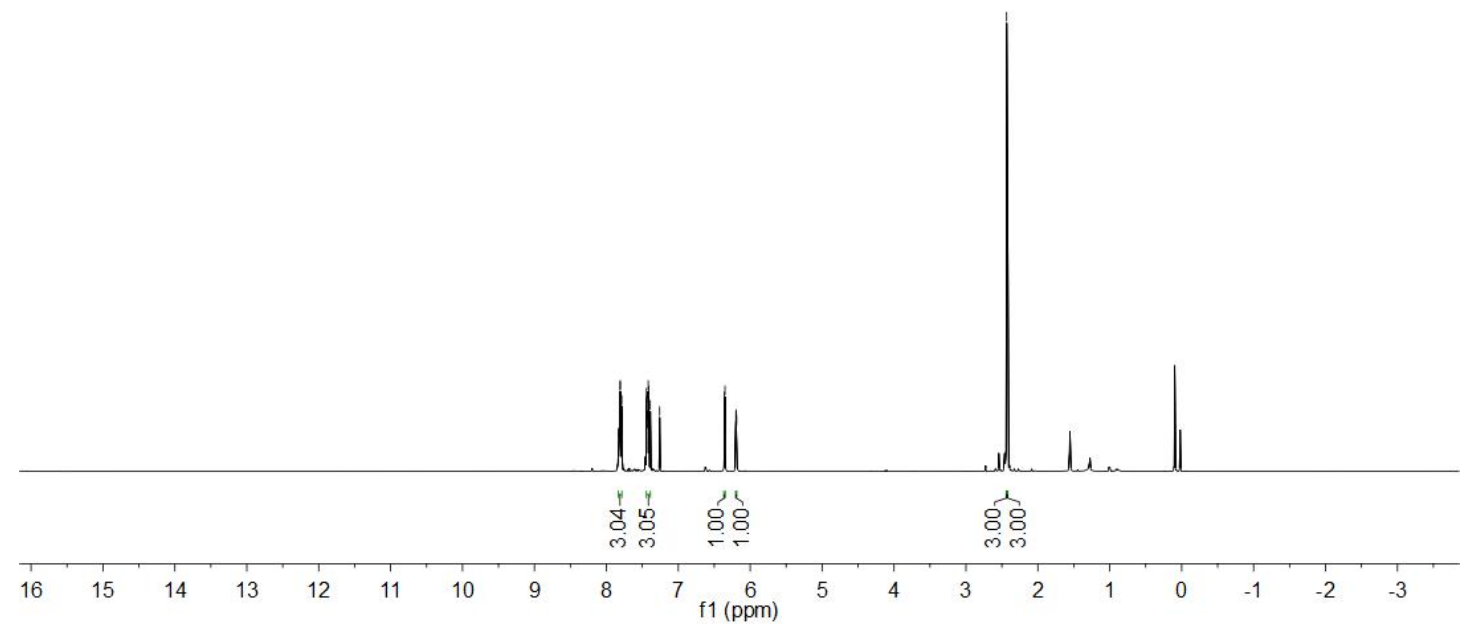

Figure S40. The ${ }^{13} \mathrm{C}$ NMR spectrums of 2-methyl-5-(2-methylnaphthalen-1-yl)furan (3an)
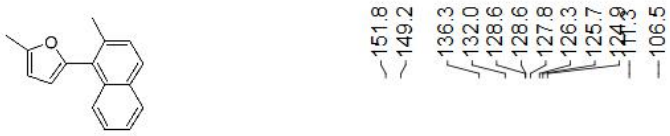

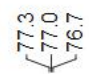

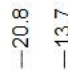

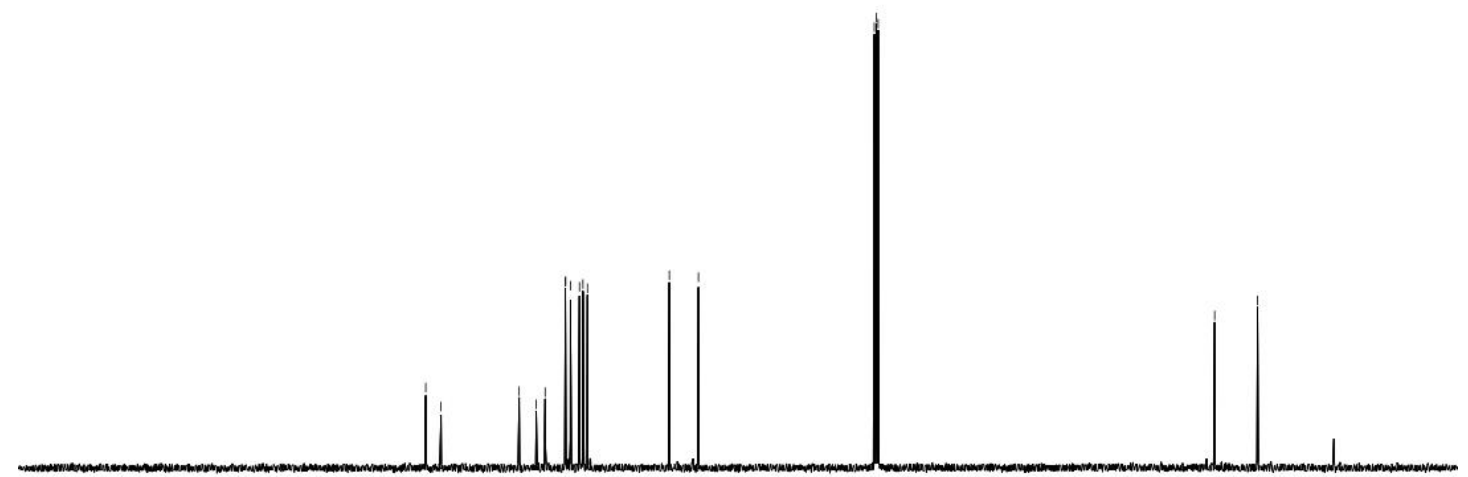

$\begin{array}{llllllllllllllllllllll}210 & 200 & 190 & 180 & 170 & 160 & 150 & 140 & 130 & 120 & 110 \begin{array}{l}100 \\ \mathrm{f} 1(\mathrm{ppm})\end{array} & 90 & 80 & 70 & 60 & 50 & 40 & 30 & 20 & 10 & 0 & -10\end{array}$ 
Figure S41. The ${ }^{1} \mathrm{H}$ NMR spectrums of 2-methyl-5-(phenanthren-9-yl)furan (3ao)

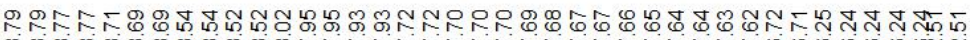

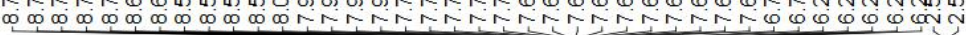
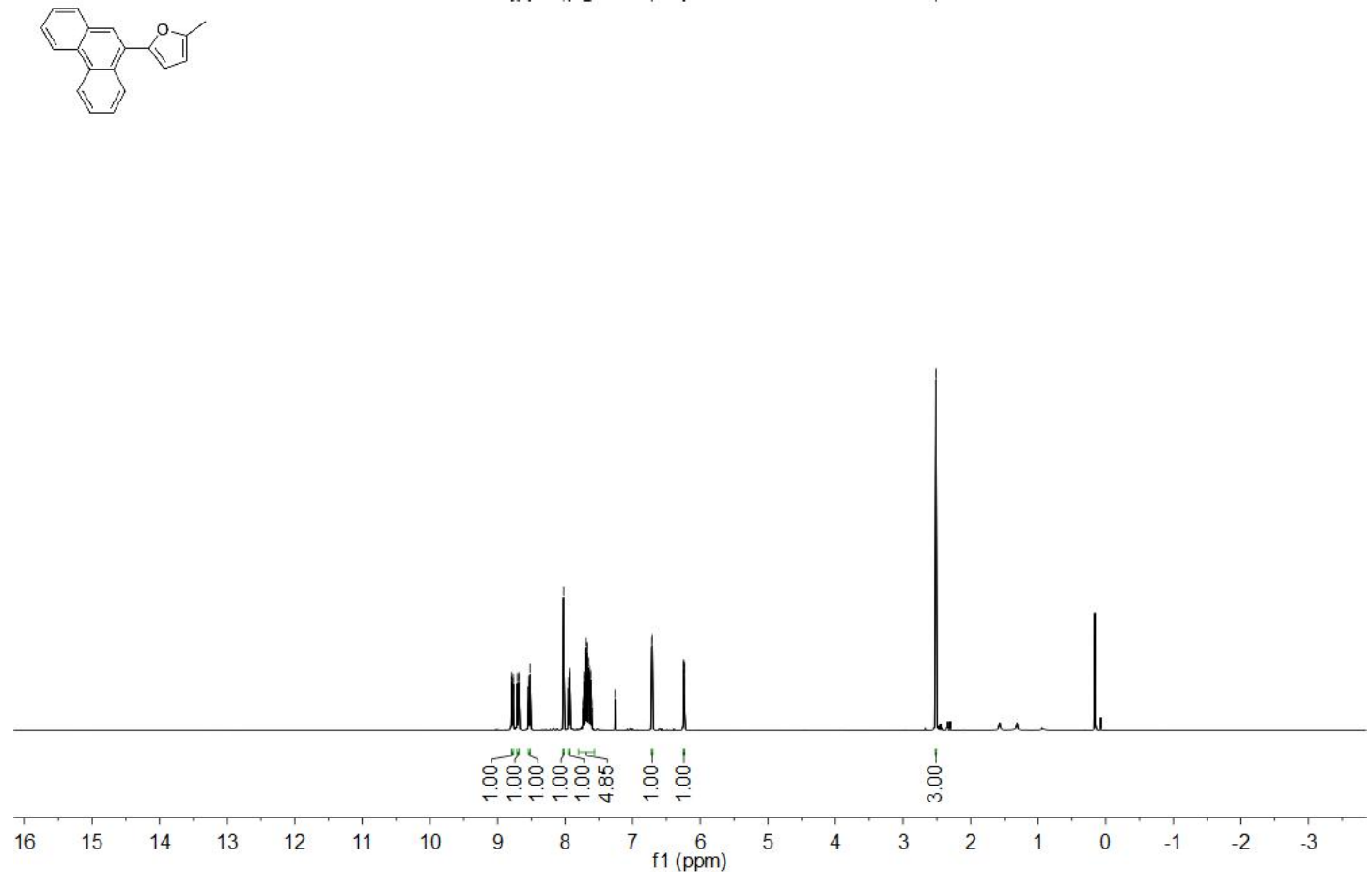

Figure S42. The ${ }^{13} \mathrm{C}$ NMR spectrums of 2-methyl-5-(phenanthren-9-yl)furan (3ao)

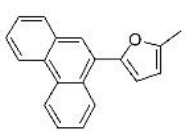

穴

证

$\stackrel{\substack{m \\ \Gamma}}{\sim}$

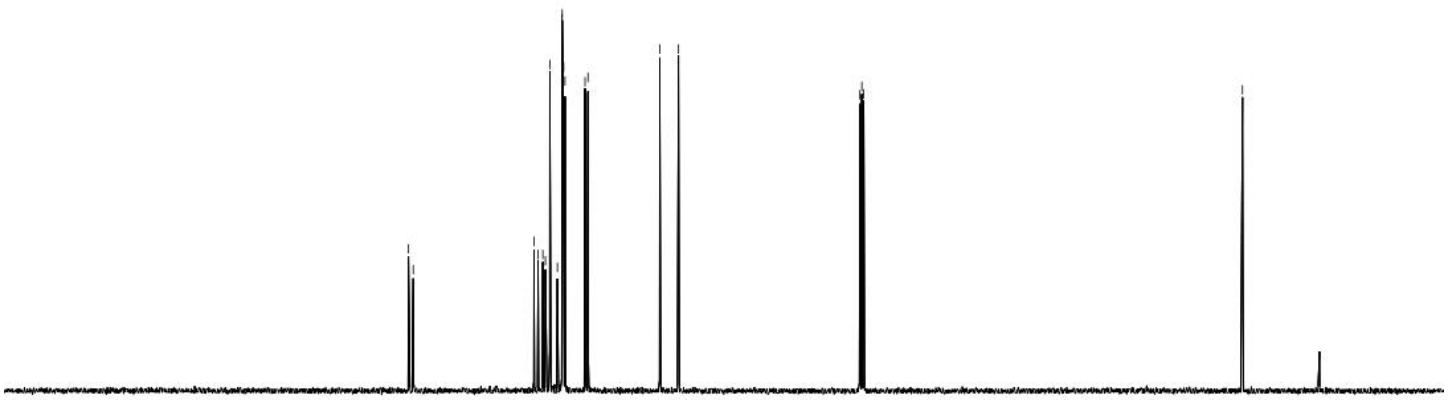

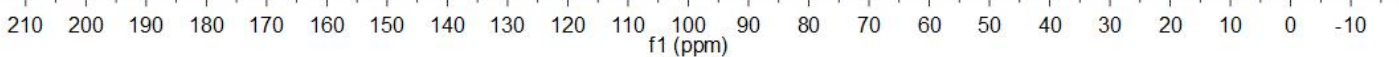


Figure S43. The ${ }^{1}$ H NMR spectrums of 2-(4-chlorophenyl)-5-pentylfuran (3be)
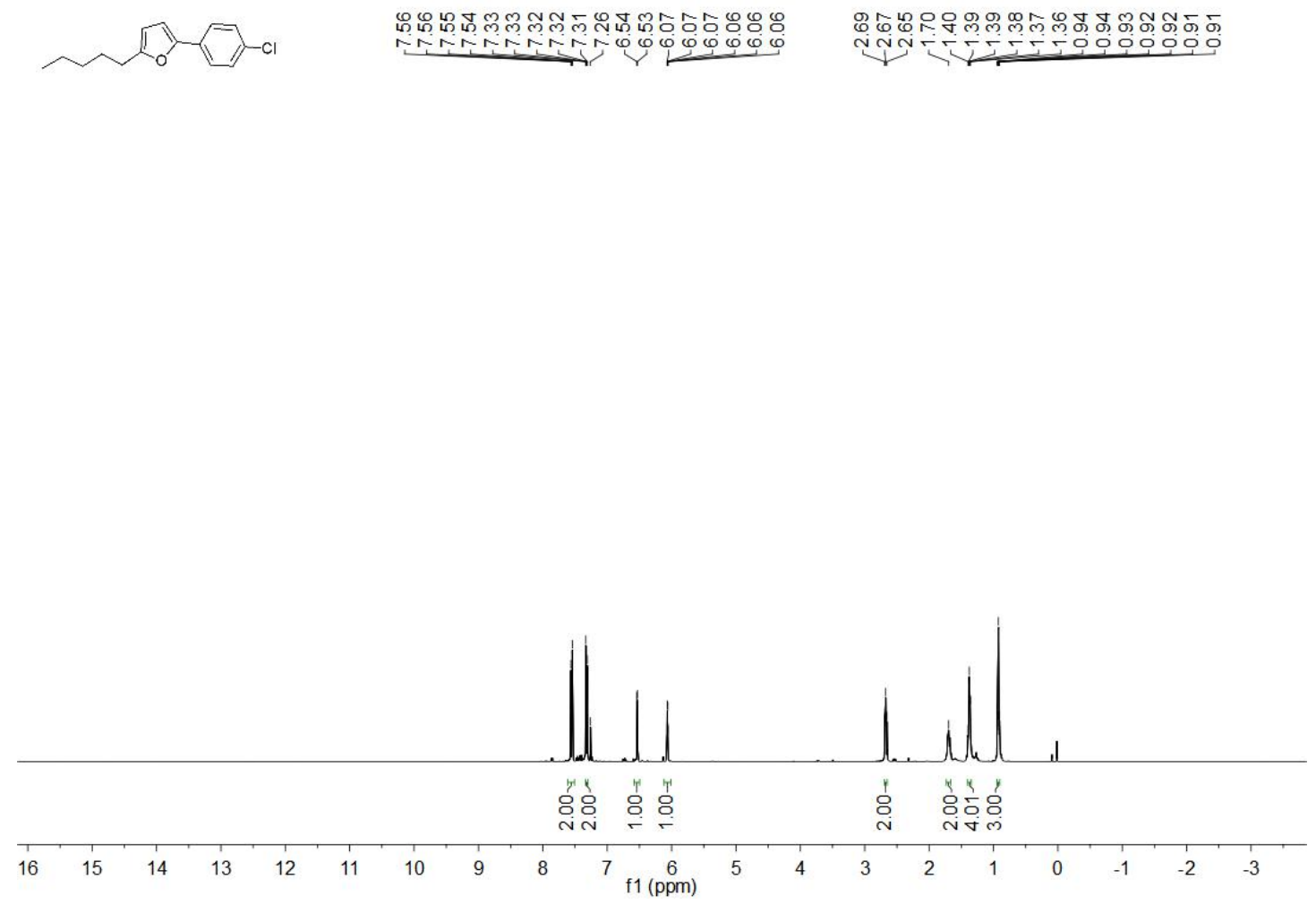

Figure S44. The ${ }^{13} \mathrm{C}$ NMR spectrums of 2-(4-chlorophenyl)-5-pentylfuran (3be)

N

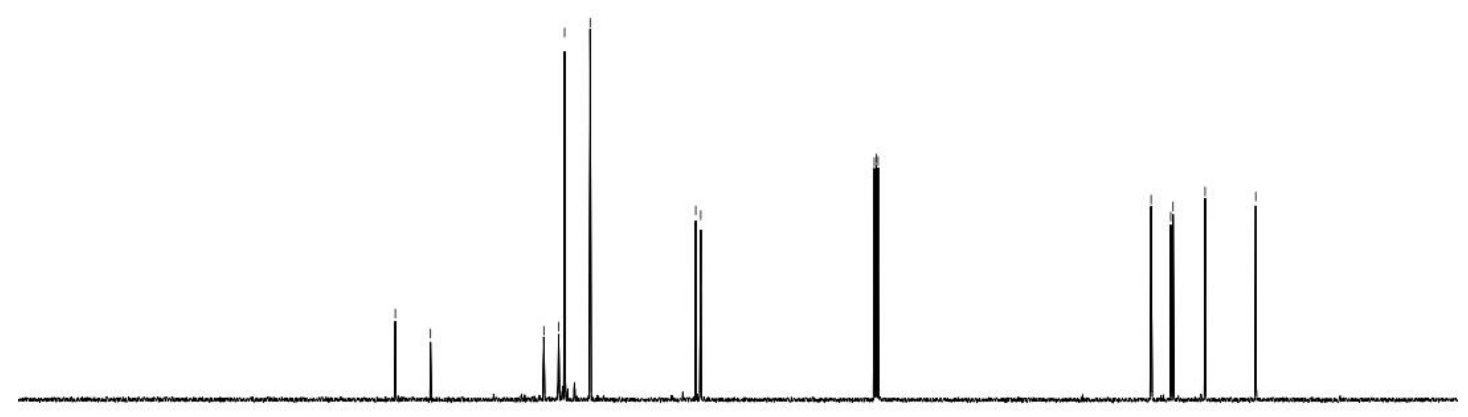

$\begin{array}{lllllllllllllllllllllll}210 & 200 & 190 & 180 & 170 & 160 & 150 & 140 & 130 & 120 & 110 & \begin{array}{c}100 \\ \mathrm{f} 1(\mathrm{ppm})\end{array} & 90 & 80 & 70 & 60 & 50 & 40 & 30 & 20 & 10 & 0 & -10\end{array}$ 
Figure $\mathbf{S 4 5}$. The ${ }^{1} \mathrm{H}$ NMR spectrums of 2-pentyl-5-(phenanthren-9-yl)furan (3bo)

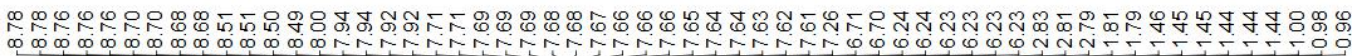

2
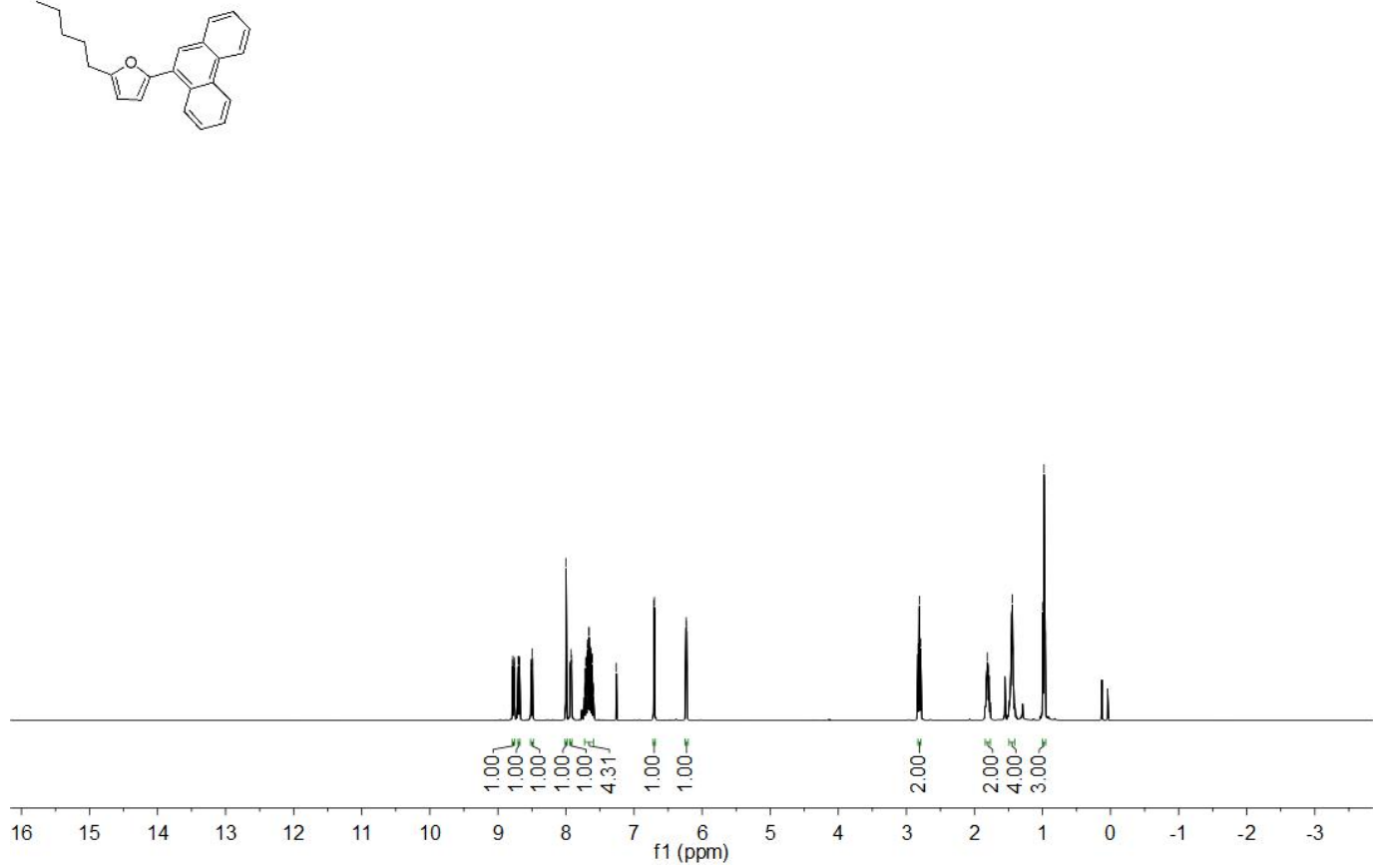

Figure S46. The ${ }^{13} \mathrm{C}$ NMR spectrums of 2-pentyl-5-(phenanthren-9-yl)furan (3bo)
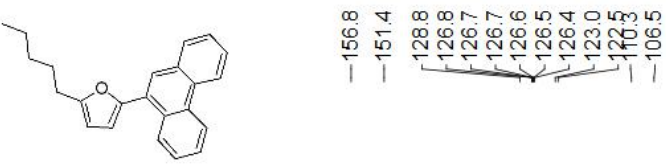

요

miñ

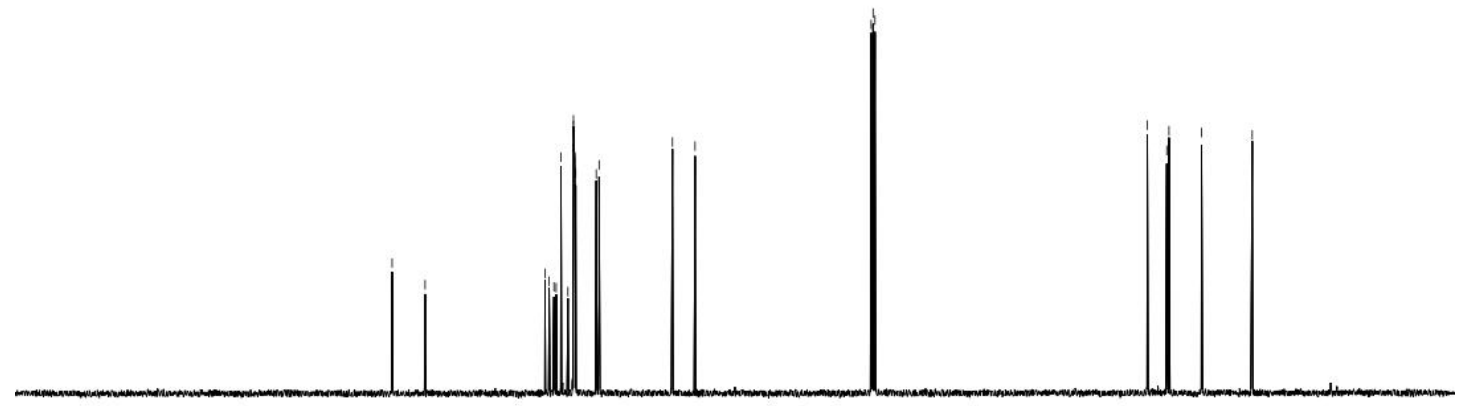

$\begin{array}{lllllllllllllllllllllll}210 & 200 & 190 & 180 & 170 & 160 & 150 & 140 & 130 & 120 & 110 & \begin{array}{c}100 \\ \mathrm{f} 1(\mathrm{ppm})\end{array} & 90 & 80 & 70 & 60 & 50 & 40 & 30 & 20 & 10 & 0 & -10\end{array}$ 
Figure S47. The ${ }^{1} \mathrm{H}$ NMR spectrums of 2-chloro-5-(5-pentylfuran-2-yl)pyridine (3bp)

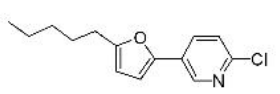

舟

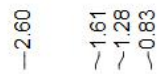

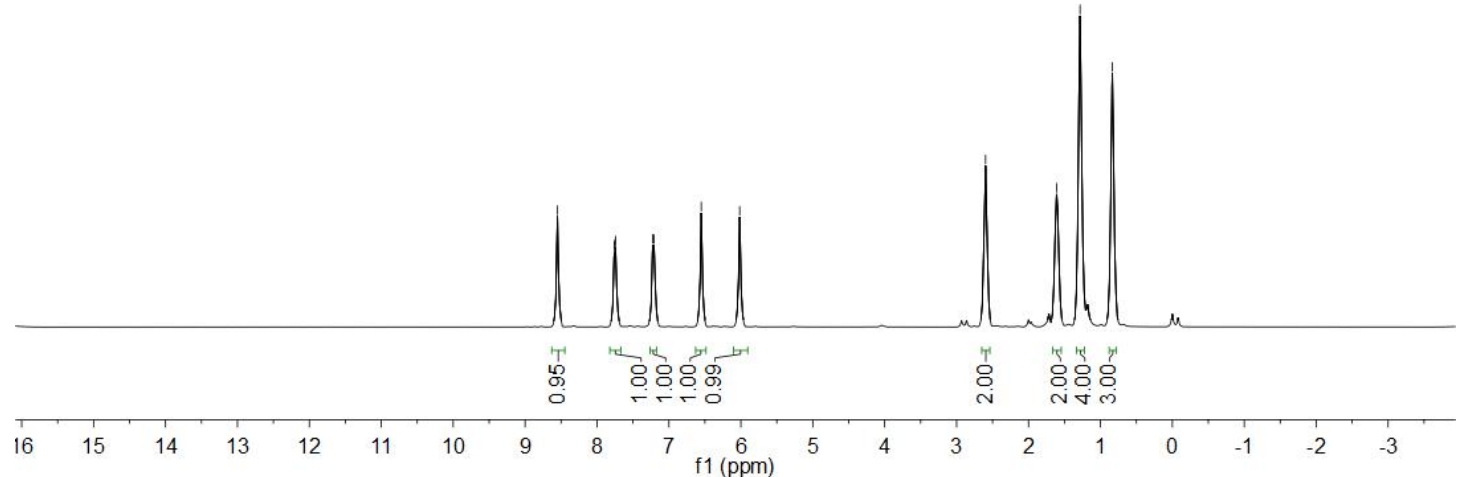

Figure S48. The ${ }^{13} \mathrm{C}$ NMR spectrums of 2-chloro-5-(5-pentylfuran-2-yl)pyridine (3bp)

यु

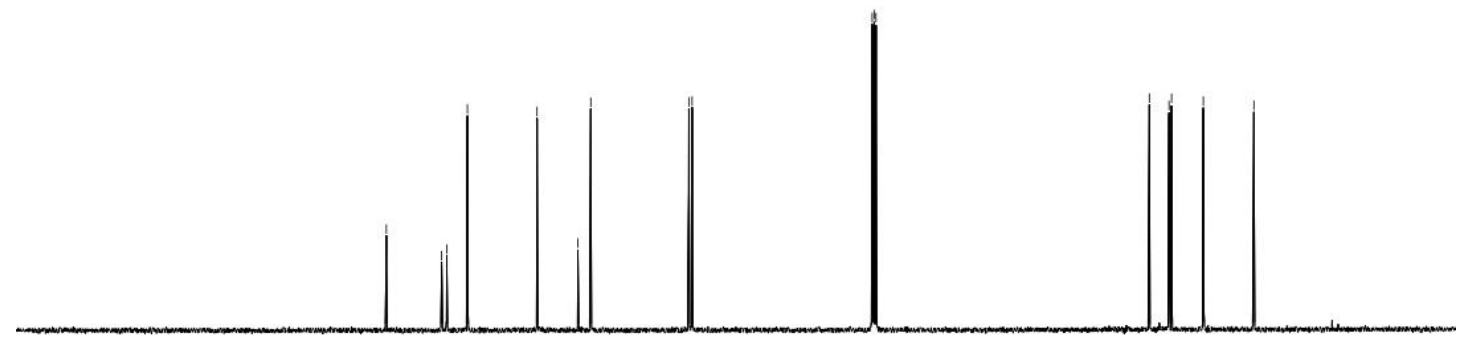

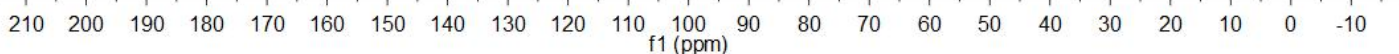


Figure S49. The ${ }^{1} \mathrm{H}$ NMR spectrums of 5-(5-pentylfuran-2-yl)pyrimidine (3bq)
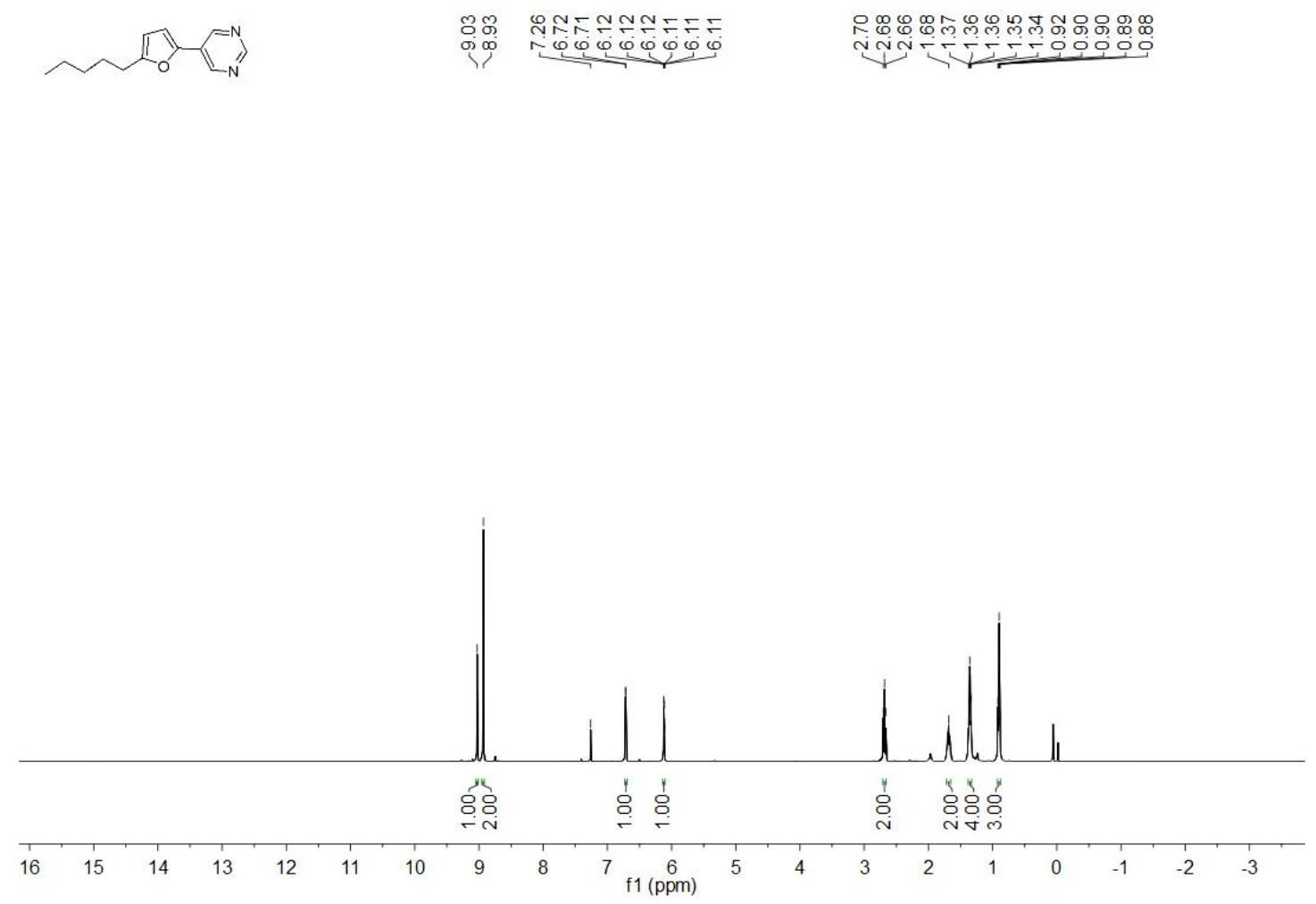

Figure S50. The ${ }^{13} \mathrm{C}$ NMR spectrums of 5-(5-pentylfuran-2-yl)pyrimidine (3bq)

N

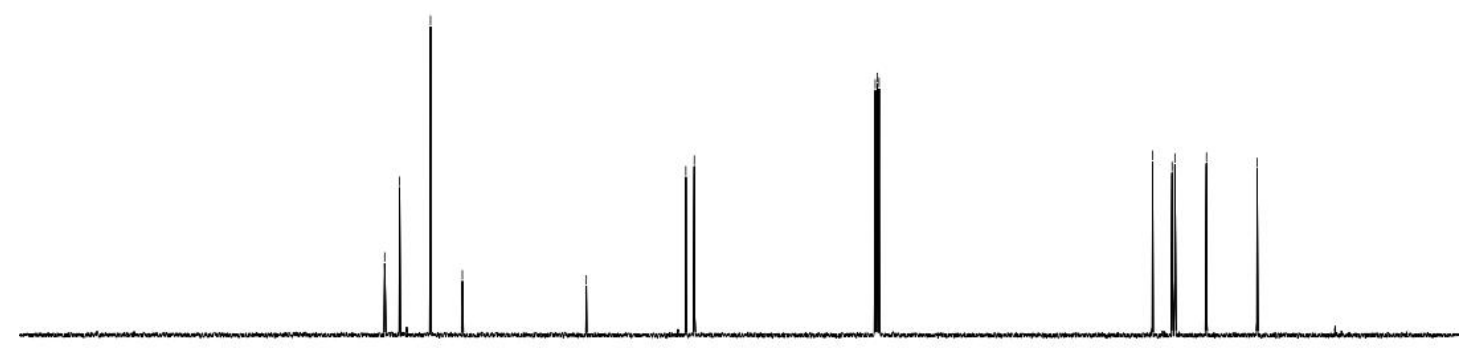

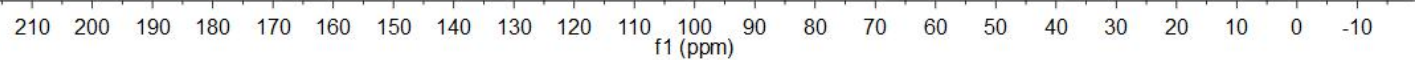


Figure S51. The ${ }^{1} \mathrm{H}$ NMR spectrums of 2-(naphthalen-2-yl)-5-pentylfuran (3br)
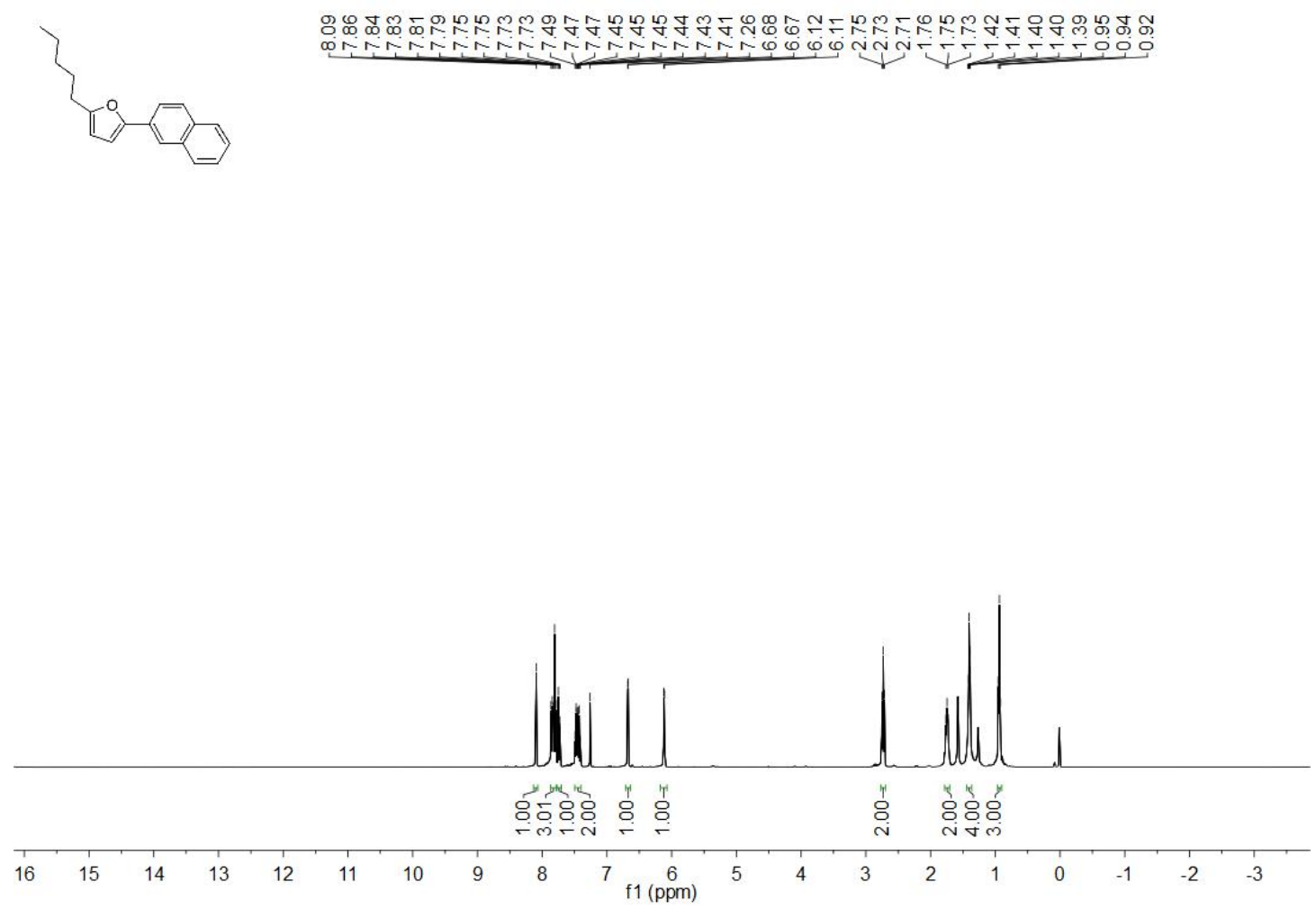

Figure S52. The ${ }^{13} \mathrm{C}$ NMR spectrums of 2-(naphthalen-2-yl)-5-pentylfuran (3br)
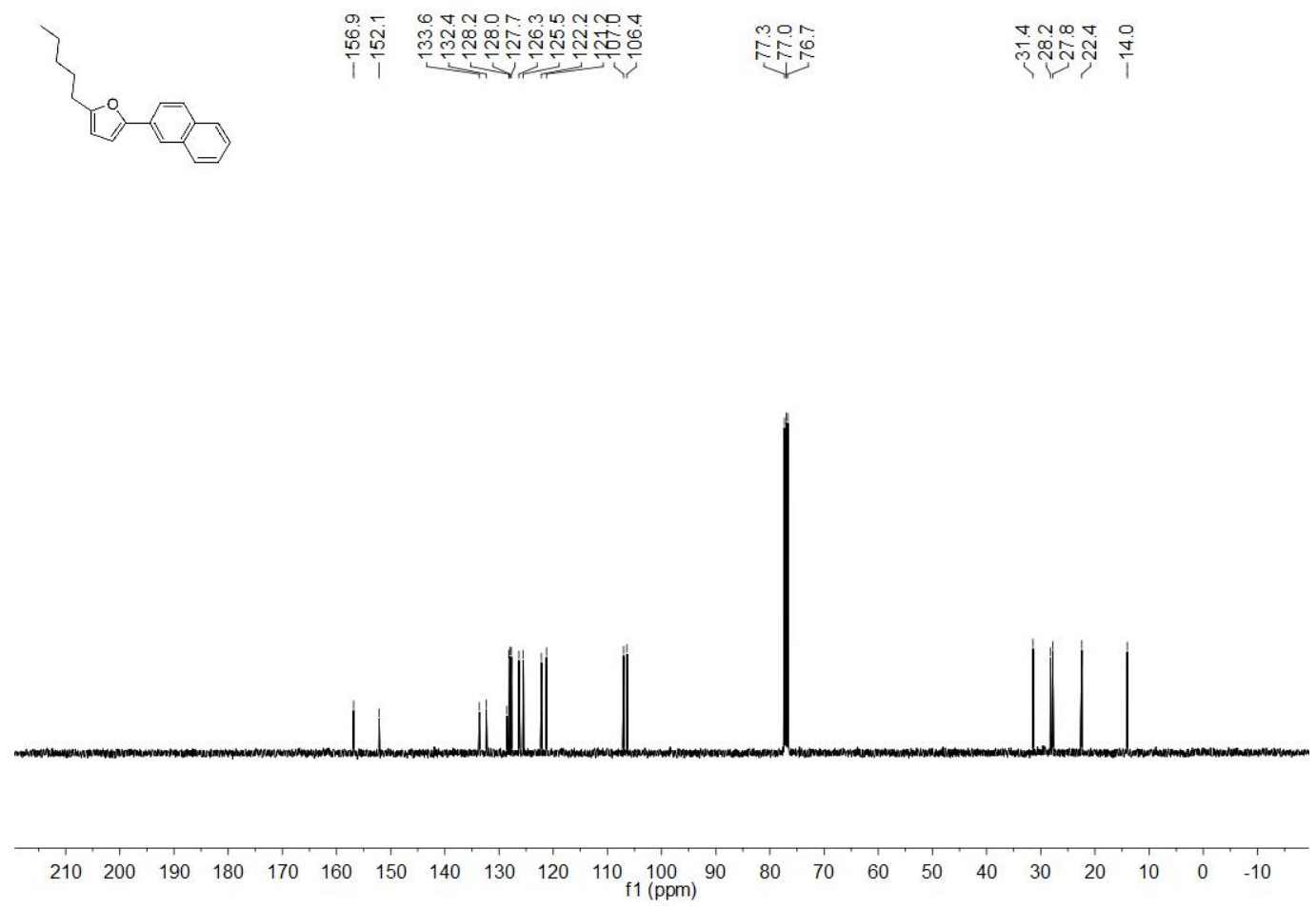
Figure S53. The ${ }^{1} \mathrm{H}$ NMR spectrums of 5-(4-nitrophenyl)furan-2-carbaldehyde (3ca)
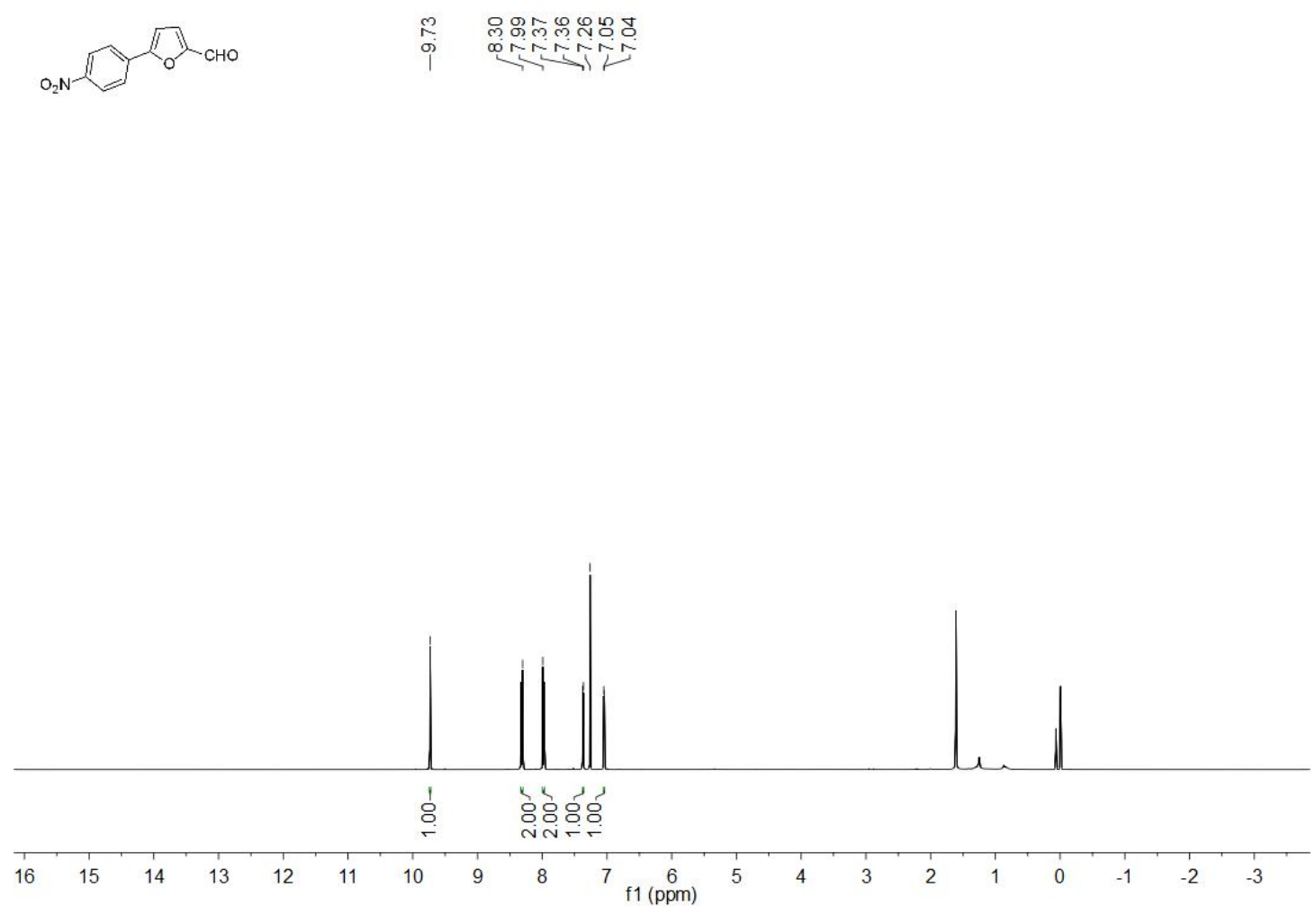

Figure S54. The ${ }^{13} \mathrm{C}$ NMR spectrums of 5-(4-nitrophenyl)furan-2-carbaldehyde (3ca)

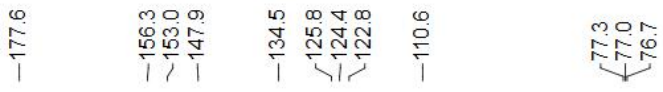<smiles>O=Cc1ccc(-c2ccc([N+](=O)[O-])cc2)o1</smiles>

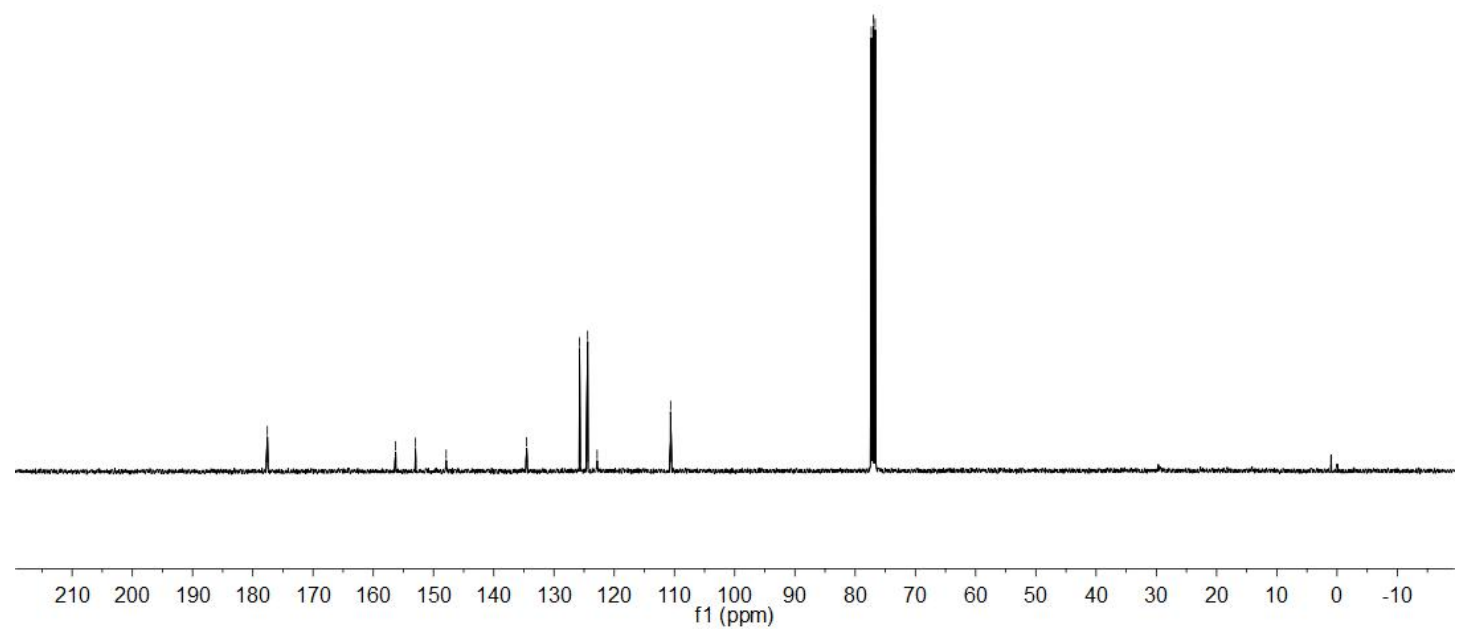


Figure S55. The ${ }^{1} \mathrm{H}$ NMR spectrums of 5-(quinolin-3-yl)furan-2-carbaldehyde (3cl)
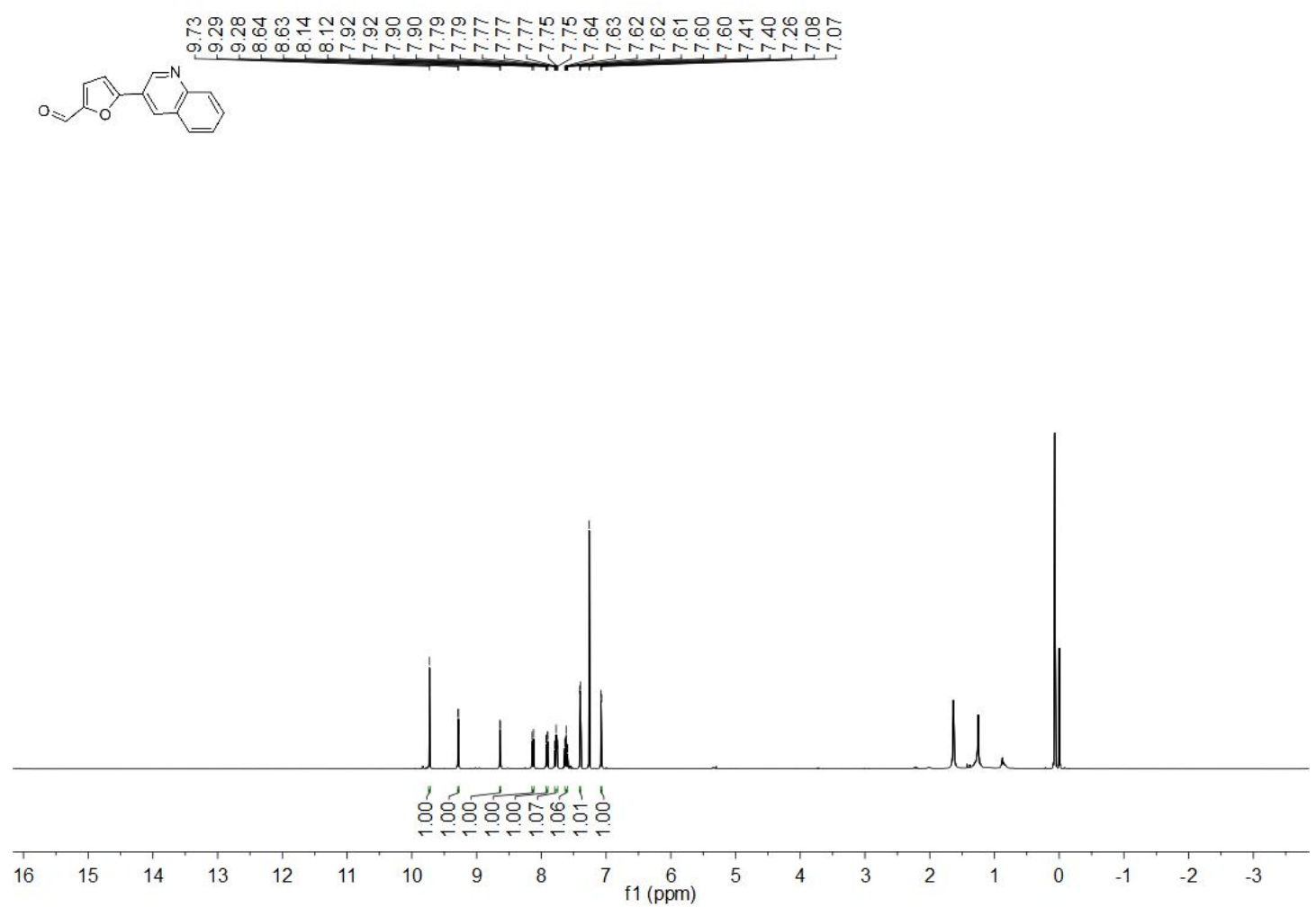

Figure S56. The ${ }^{13} \mathrm{C}$ NMR spectrums of 5-(quinolin-3-yl)furan-2-carbaldehyde (3cl)
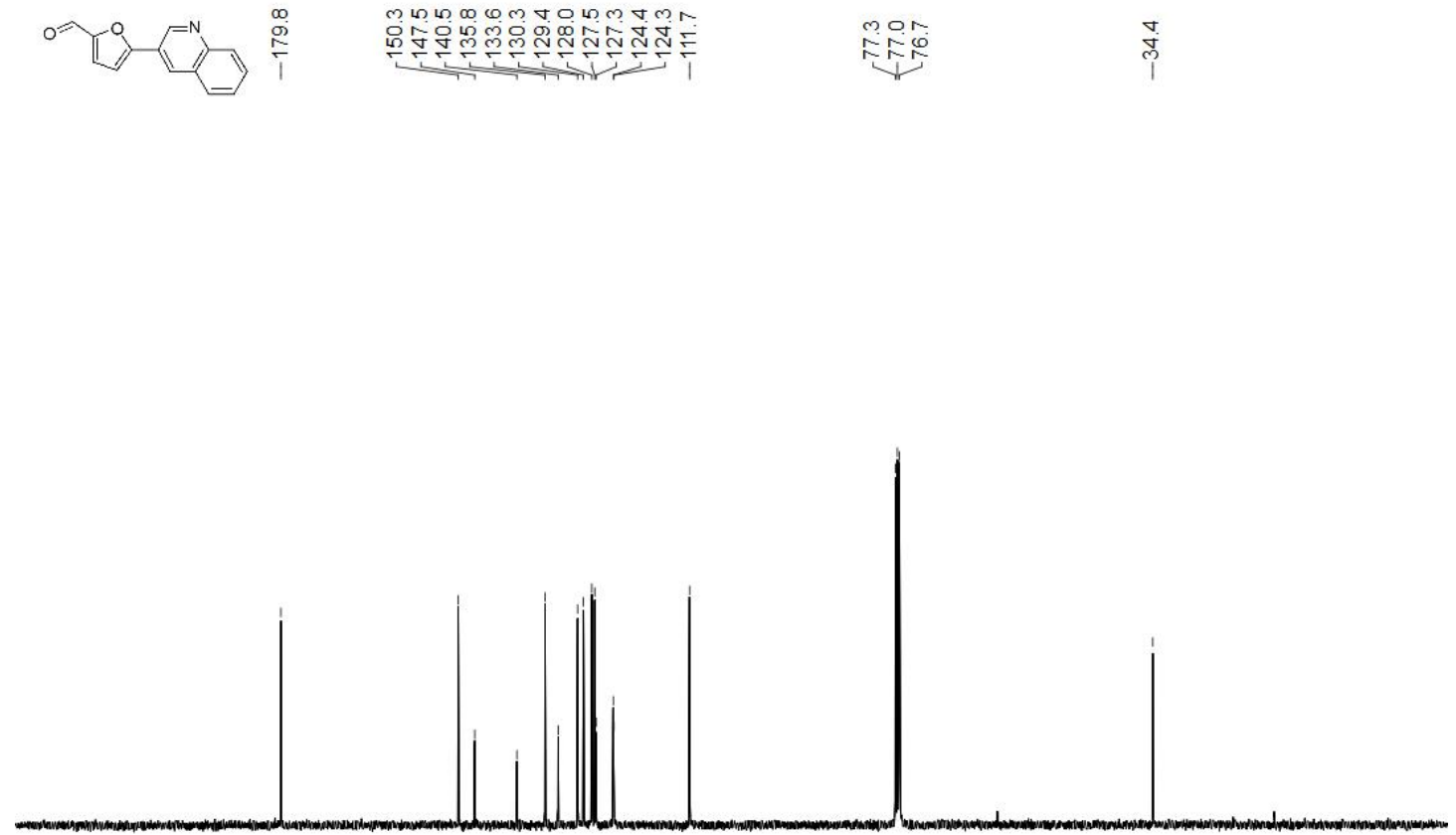

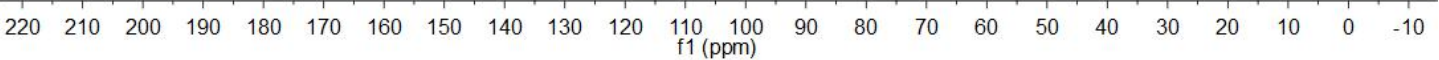


Figure S57. The ${ }^{1} \mathrm{H}$ NMR spectrums of 5-(6-methoxypyridin-3-yl)furan-2-carbaldehyde (3cs)

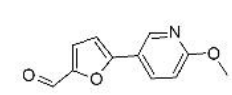

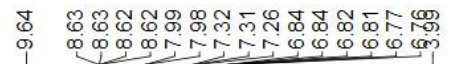

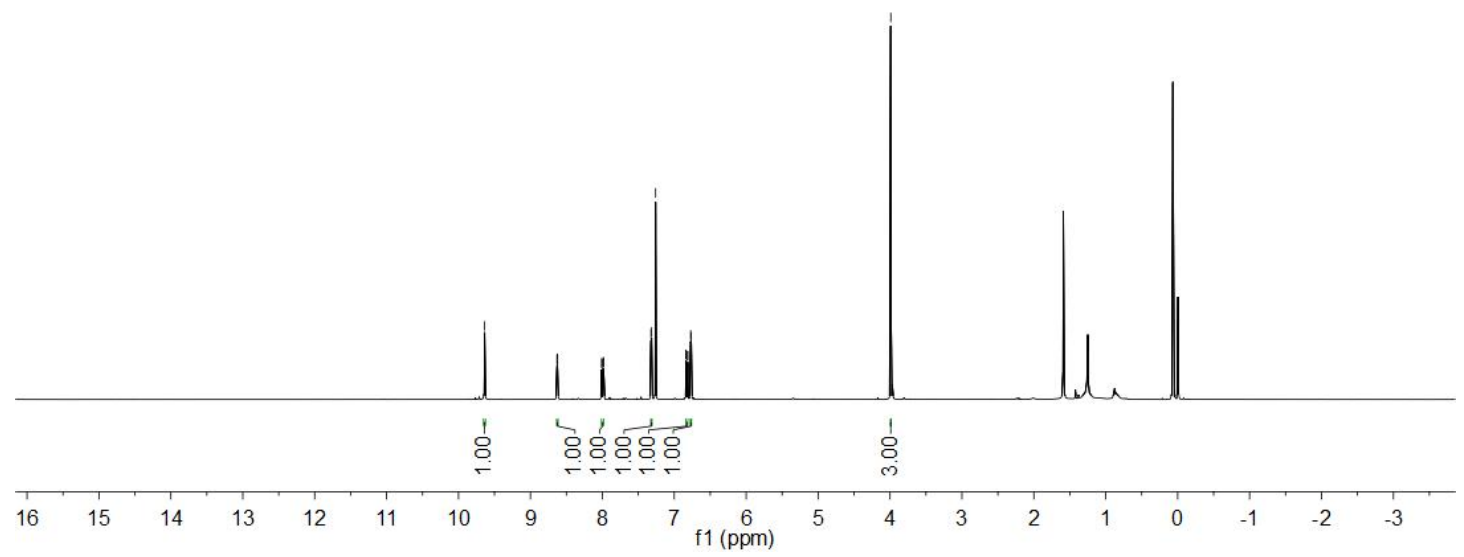

Figure S58. The ${ }^{13} \mathrm{C}$ NMR spectrums of 5-(6-methoxypyridin-3-yl)furan-2-carbaldehyde (3cs)

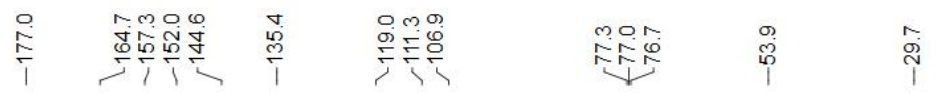
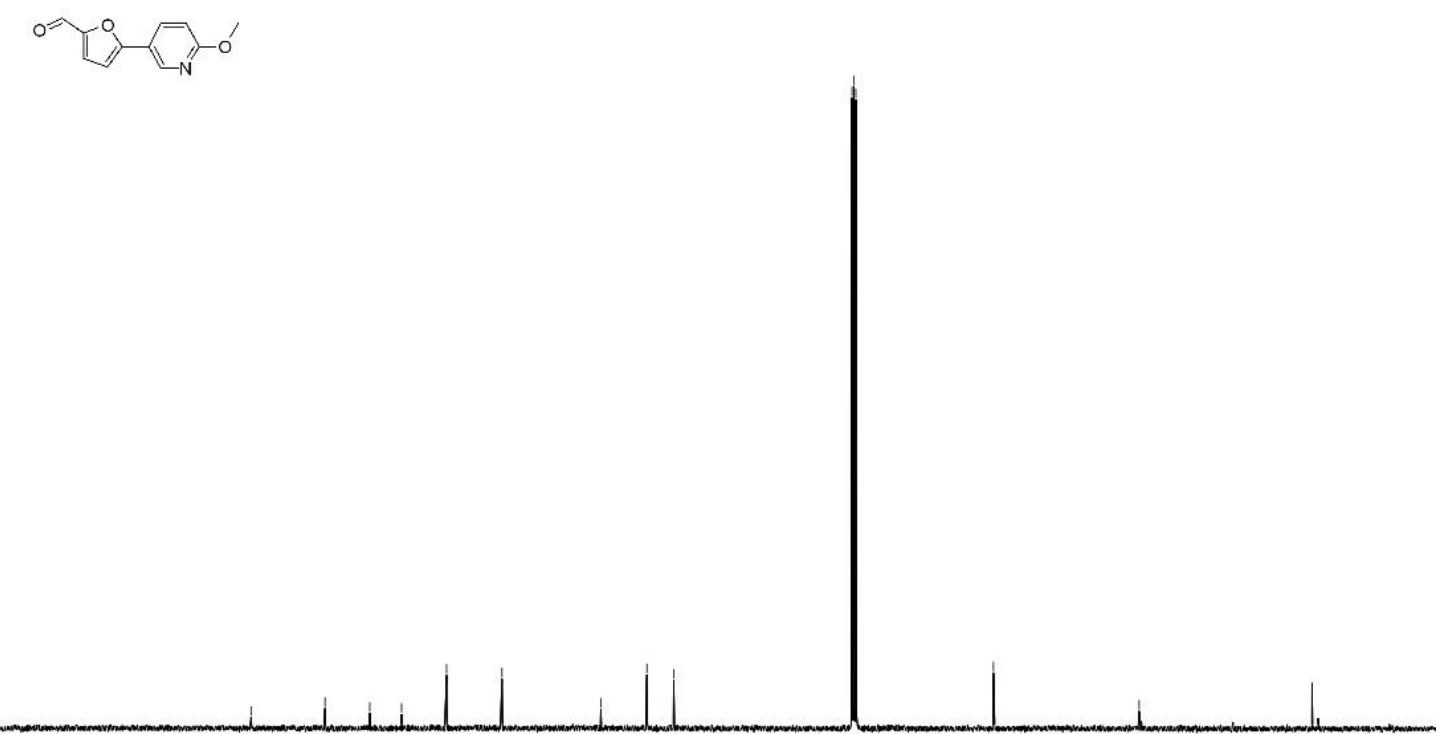

$\begin{array}{lllllllllllllllllllllll}210 & 200 & 190 & 180 & 170 & 160 & 150 & 140 & 130 & 120 & 110 & \begin{array}{c}100 \\ \mathrm{f} 1(\mathrm{ppm})\end{array} & 90 & 80 & 70 & 60 & 50 & 40 & 30 & 20 & 10 & 0 & -10\end{array}$ 
Figure S59. The ${ }^{1} \mathrm{H}$ NMR spectrums of methyl 2-methyl-5-phenylfuran-3-carboxylate (3df)
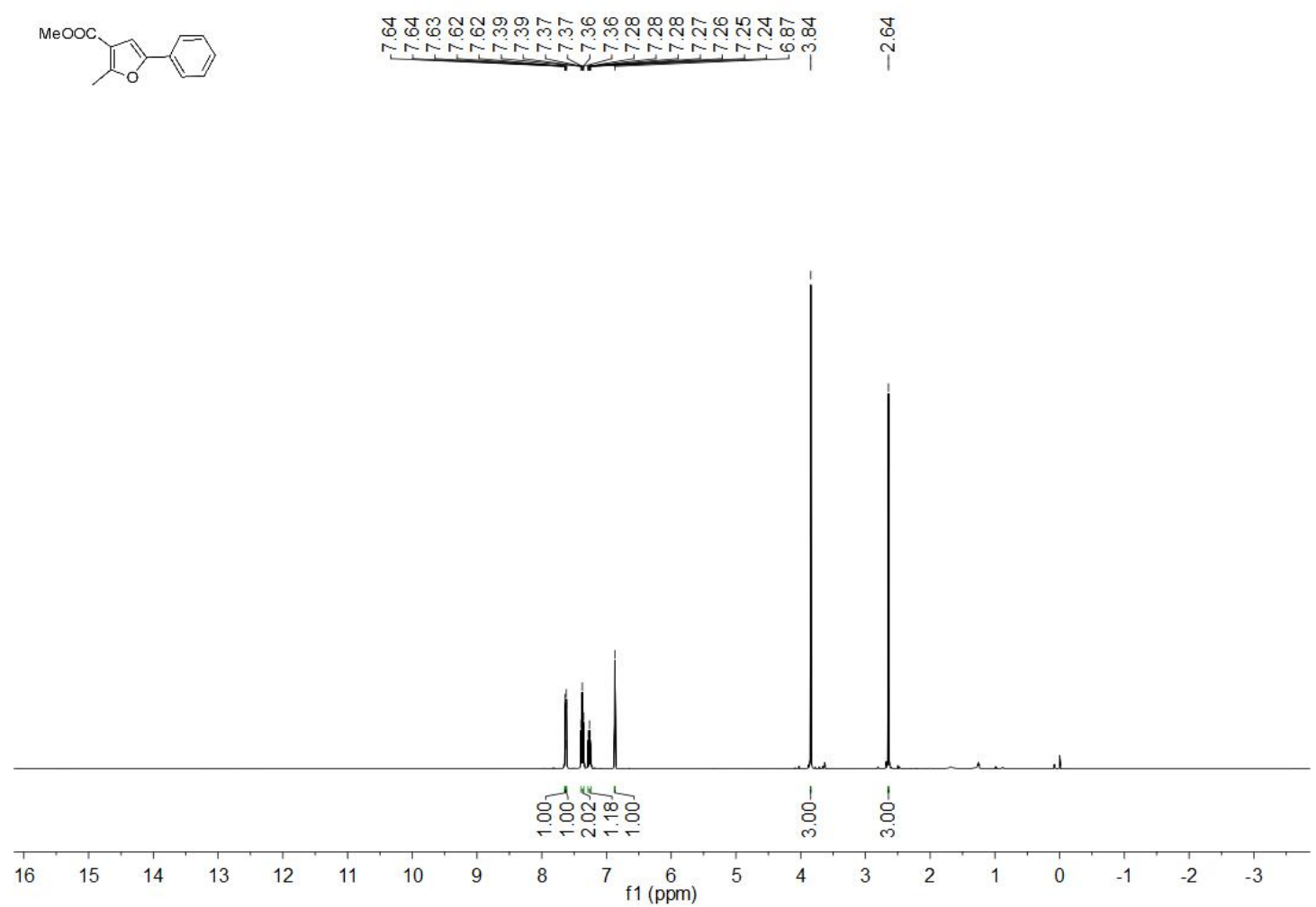

Figure S60. The ${ }^{13} \mathrm{C}$ NMR spectrums of methyl 2-methyl-5-phenylfuran-3-carboxylate (3df)

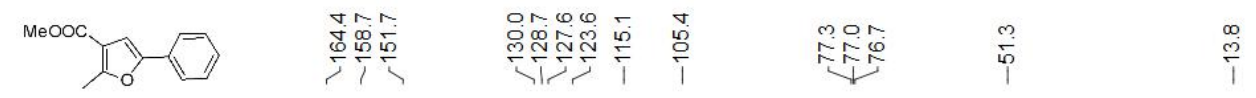

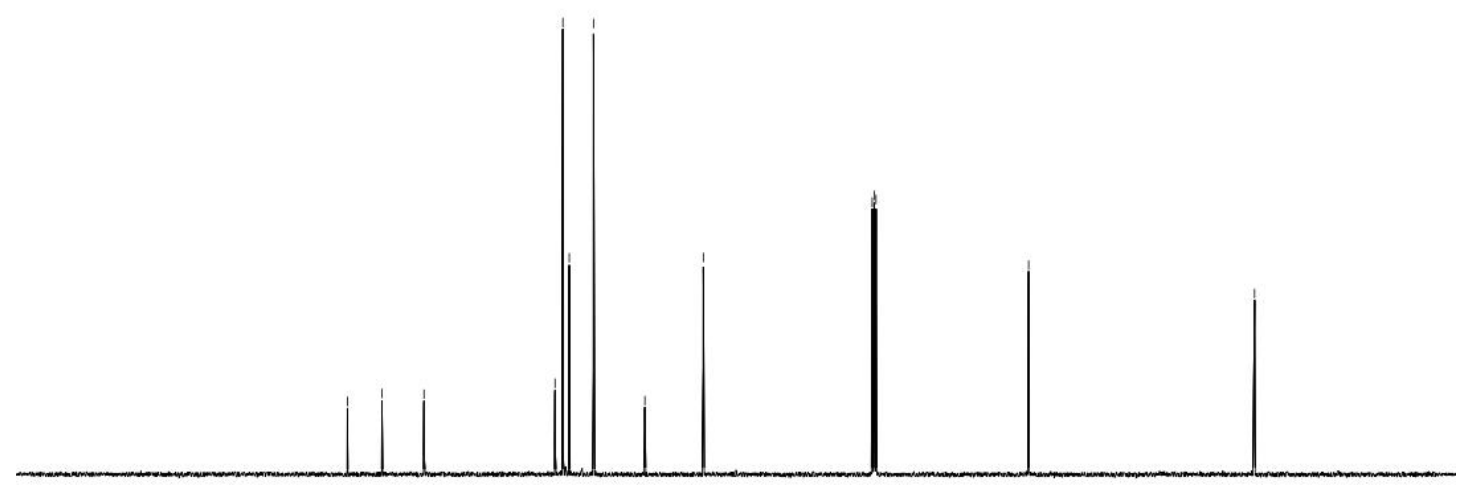

$\begin{array}{llllllllllllllllllllll}210 & 200 & 190 & 180 & 170 & 160 & 150 & 140 & 130 & 120 & 110 \begin{array}{c}100 \\ \mathrm{f} 1(\mathrm{ppm})\end{array} & 90 & 80 & 70 & 60 & 50 & 40 & 30 & 20 & 10 & 0 & -10\end{array}$ 
Figure S61. The ${ }^{1} \mathrm{H}$ NMR spectrums of methyl

5-(6-fluoropyridin-3-yl)-2-methylfuran-3-carboxylate (3dh)
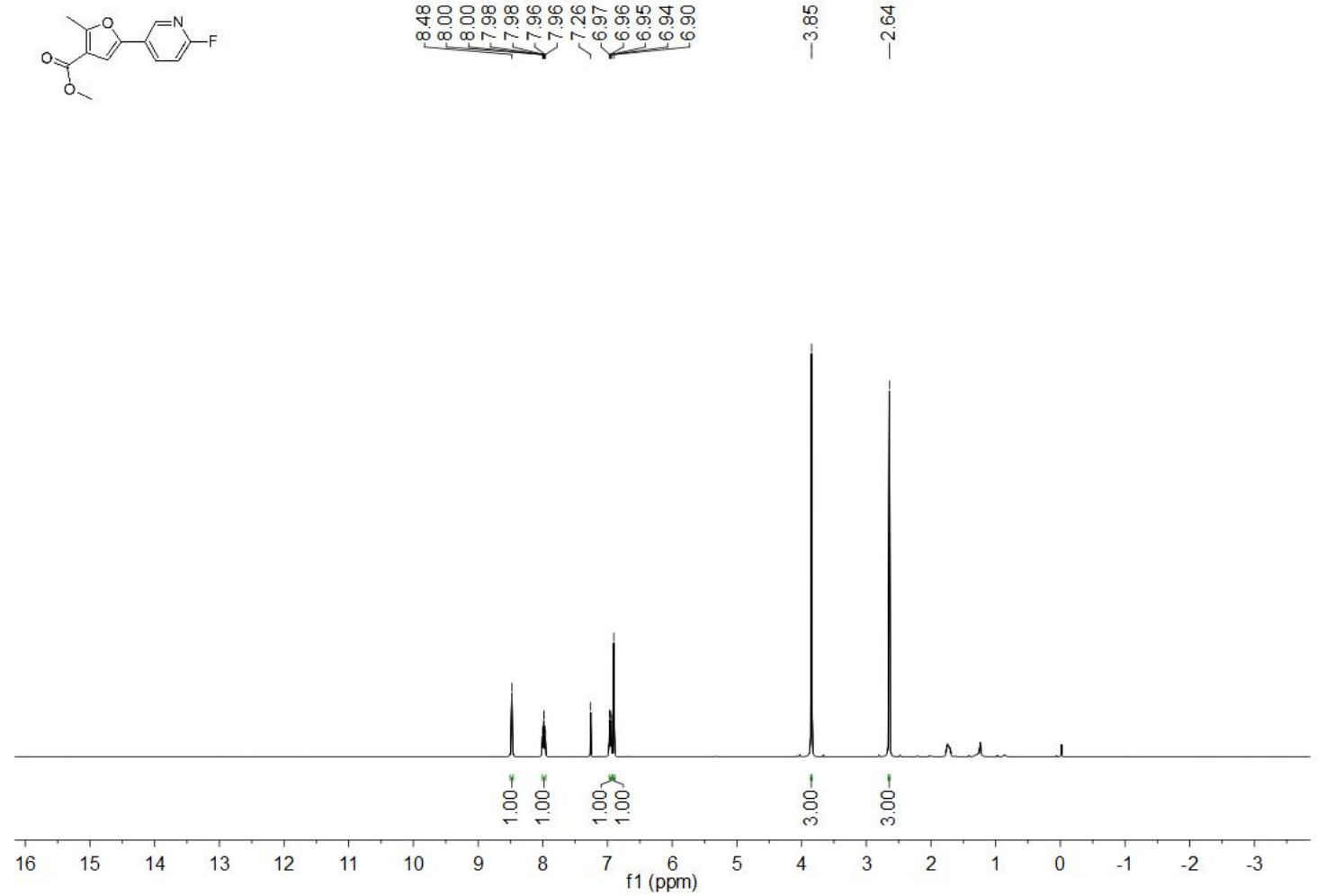

Figure S62. The ${ }^{13} \mathrm{C}$ NMR spectrums of methyl

5-(6-fluoropyridin-3-yl)-2-methylfuran-3-carboxylate (3dh)
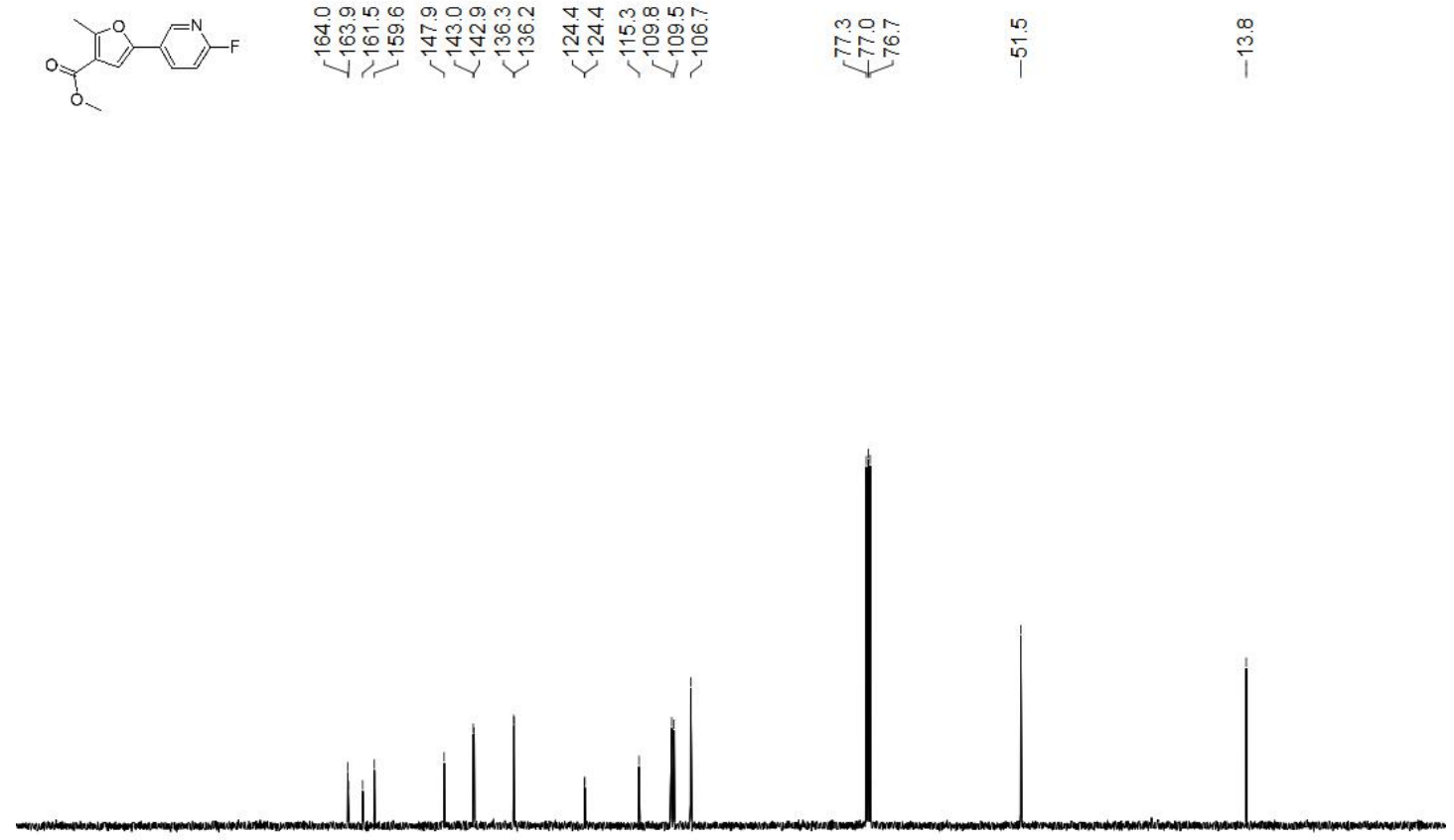

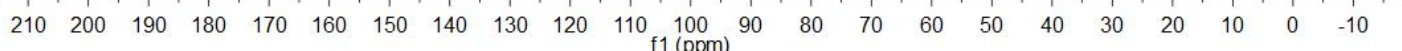


Figure S63. The ${ }^{1}$ H NMR spectrums of methyl 5-(isoquinolin-4-yl)-2-methylfuran-3-carboxylate (3dk)
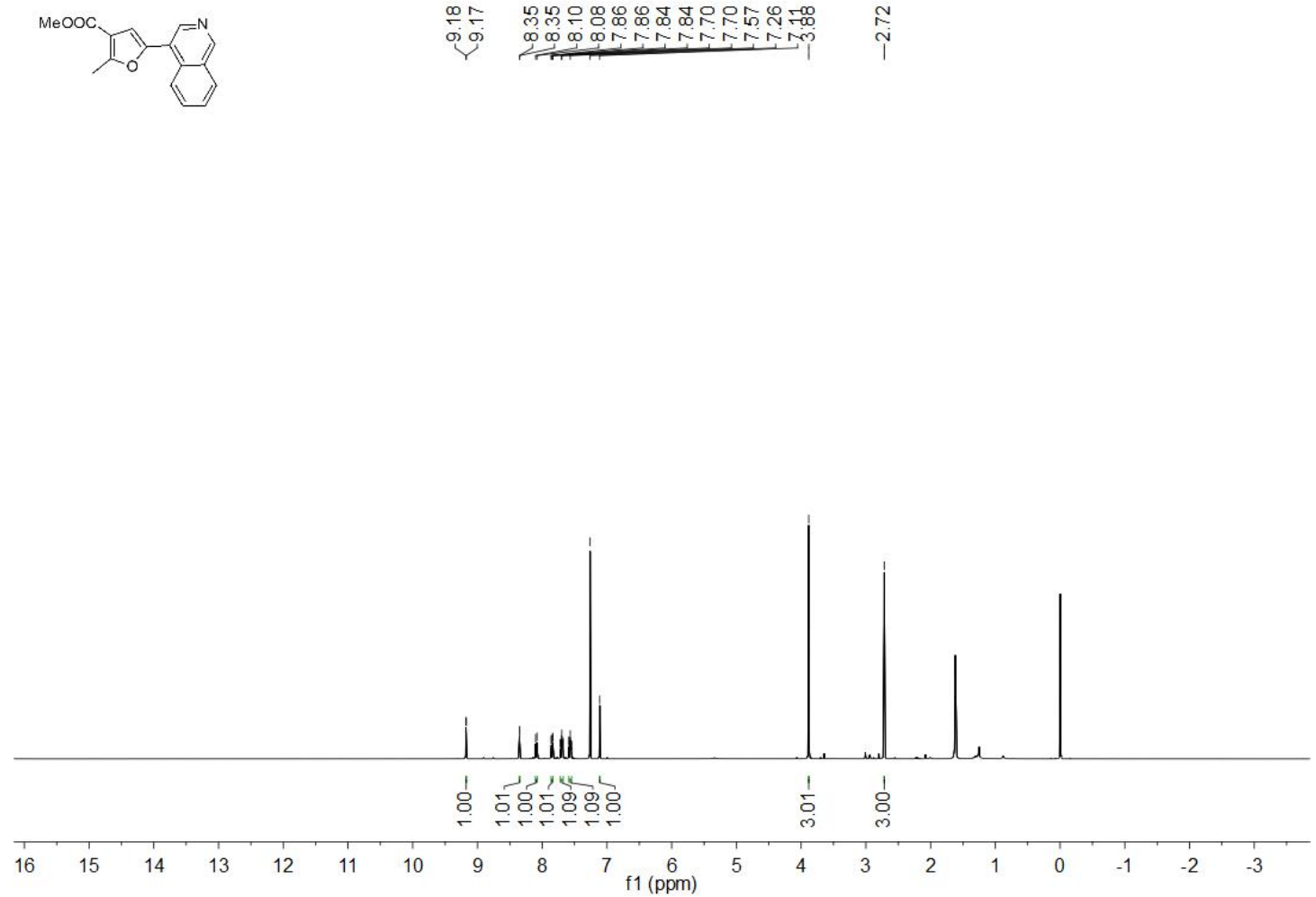

Figure S64. The ${ }^{13} \mathrm{C}$ NMR spectrums of methyl 5-(isoquinolin-4-yl)-2-methylfuran-3-carboxylate (3dk)

MeOcc

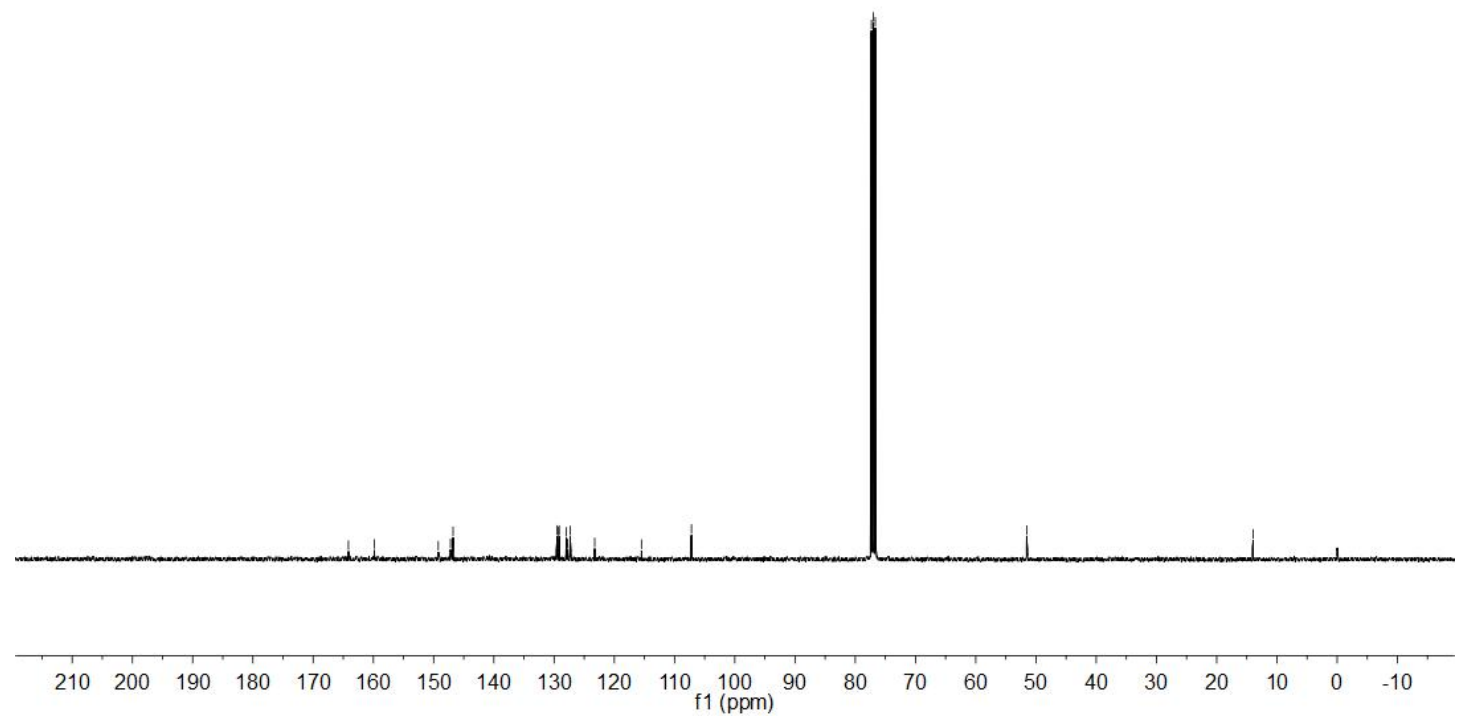


Figure S65. The ${ }^{1} \mathrm{H}$ NMR spectrums of methyl

2-methyl-5-(2-methylnaphthalen-1-yl)furan-3-carboxylate (3dn)

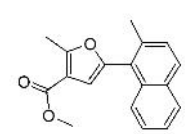

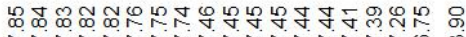

andine m

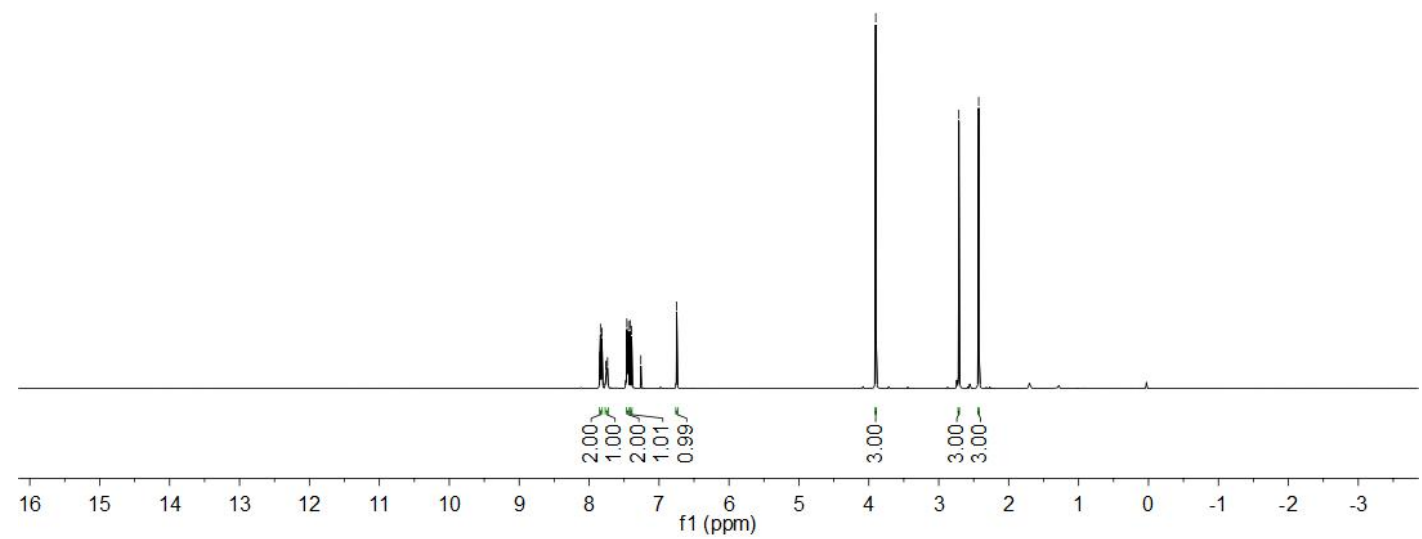

Figure S66. The ${ }^{13} \mathrm{C}$ NMRspectrums of methyl

2-methyl-5-(2-methylnaphthalen-1-yl)furan-3-carboxylate (3dn)

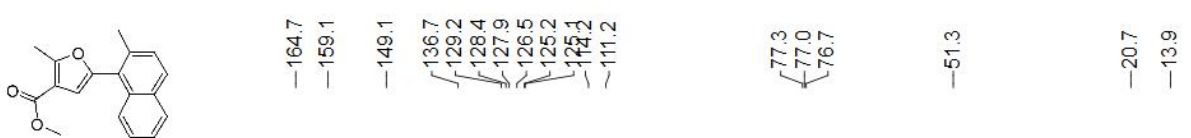

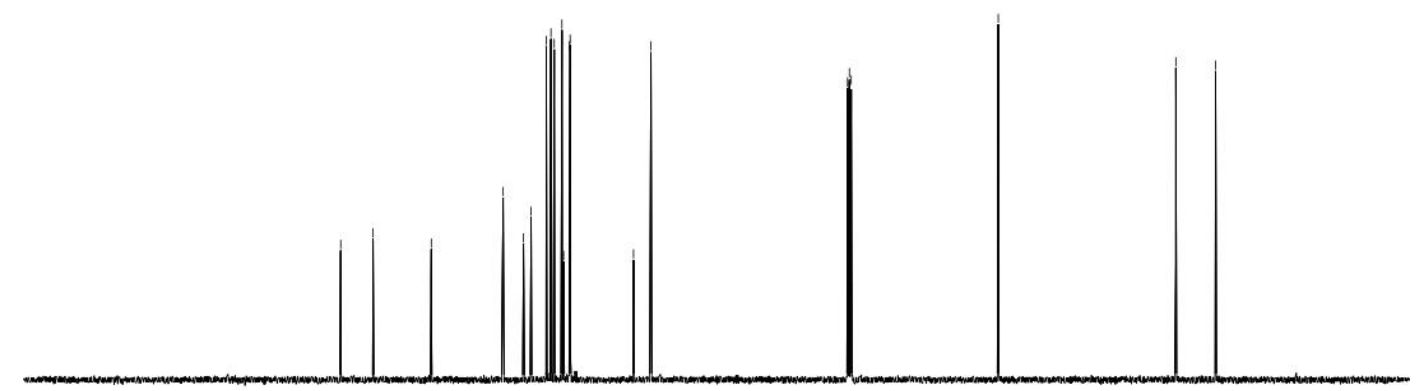

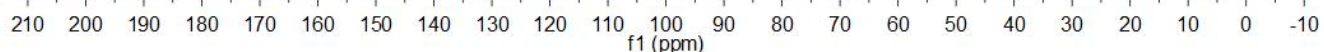


Figure S67. The ${ }^{1} \mathrm{H}$ NMR spectrums of 5-(6-methoxypyridin-3-yl)furan-2-carbaldehyde (3ds)<smiles>COC(=O)c1cc(-c2ccc(OC)nc2)oc1C</smiles>

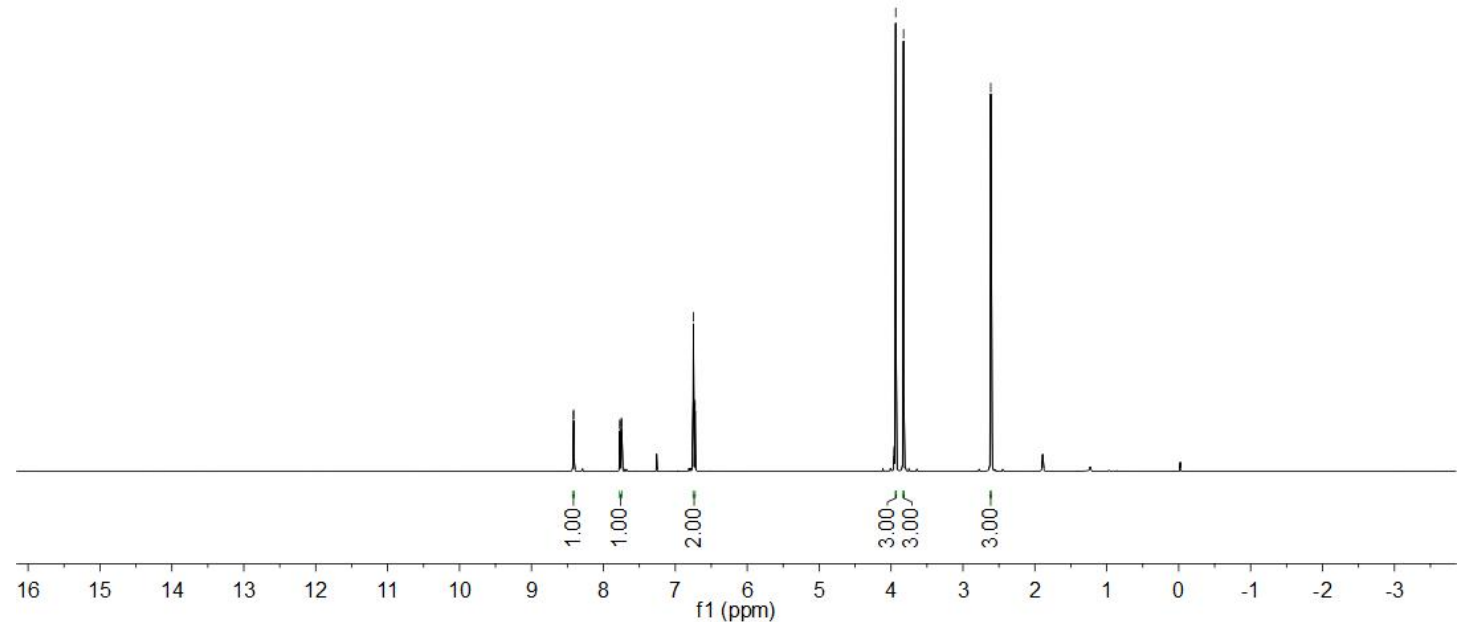

Figure S68. The ${ }^{13} \mathrm{C}$ NMR spectrums of 5-(6-methoxypyridin-3-yl)furan-2-carbaldehyde (3ds)

O.

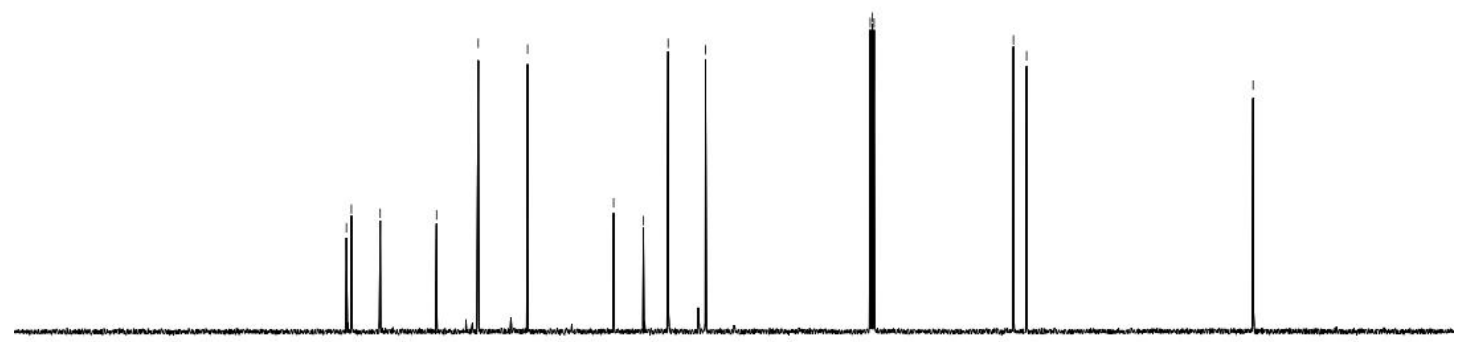

$\begin{array}{llllllllllllllllllllll}210 & 200 & 190 & 180 & 170 & 160 & 150 & 140 & 130 & 120 & 110 \begin{array}{l}100 \\ \mathrm{f} 1(\mathrm{ppm})\end{array} & 90 & 80 & 70 & 60 & 50 & 40 & 30 & 20 & 10 & 0 & -10\end{array}$ 
Figure S69. The ${ }^{1} \mathrm{H}$ NMR spectrums of methyl

5-(4-(methoxycarbonyl)phenyl)-2-methylfuran-3-carboxylate (3dt)
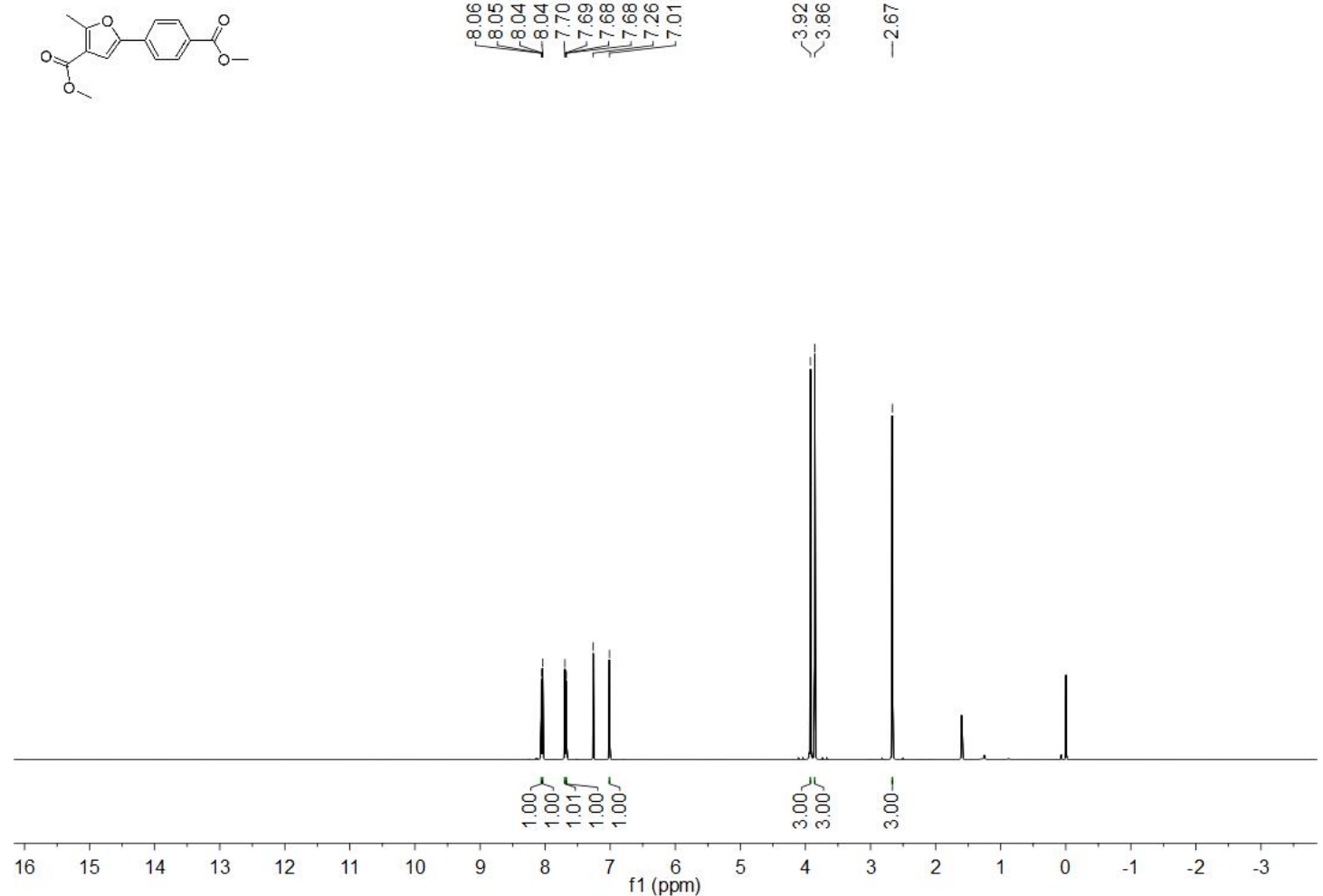

Figure S70. The ${ }^{13} \mathrm{C}$ NMR spectrums of methyl

5-(4-(methoxycarbonyl)phenyl)-2-methylfuran-3-carboxylate (3dt)
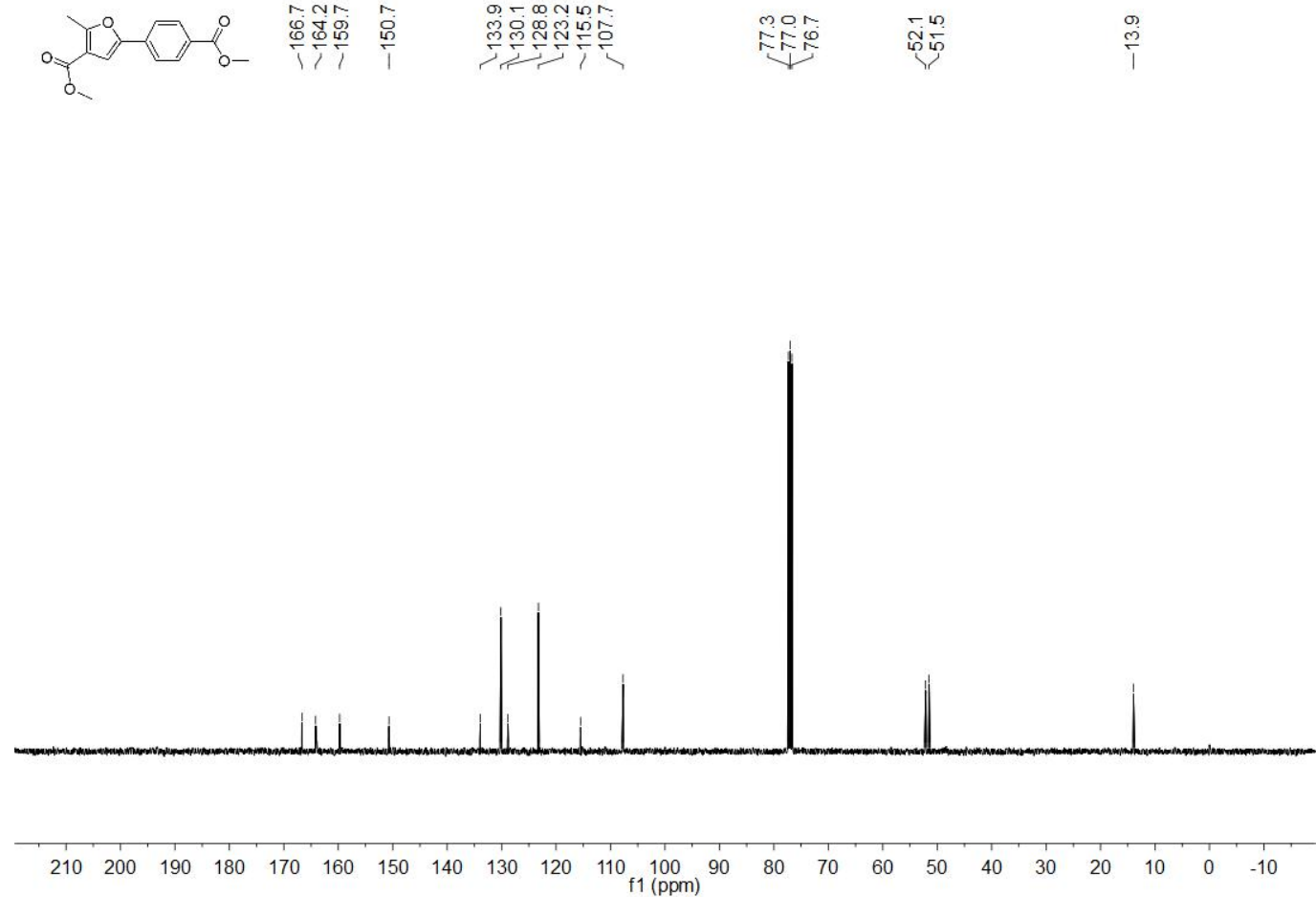
Figure S71. The ${ }^{1} \mathrm{H}$ NMR spectrums of 2-(4-nitrophenyl)benzofuran (3ea)

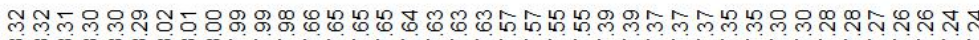

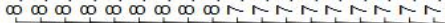
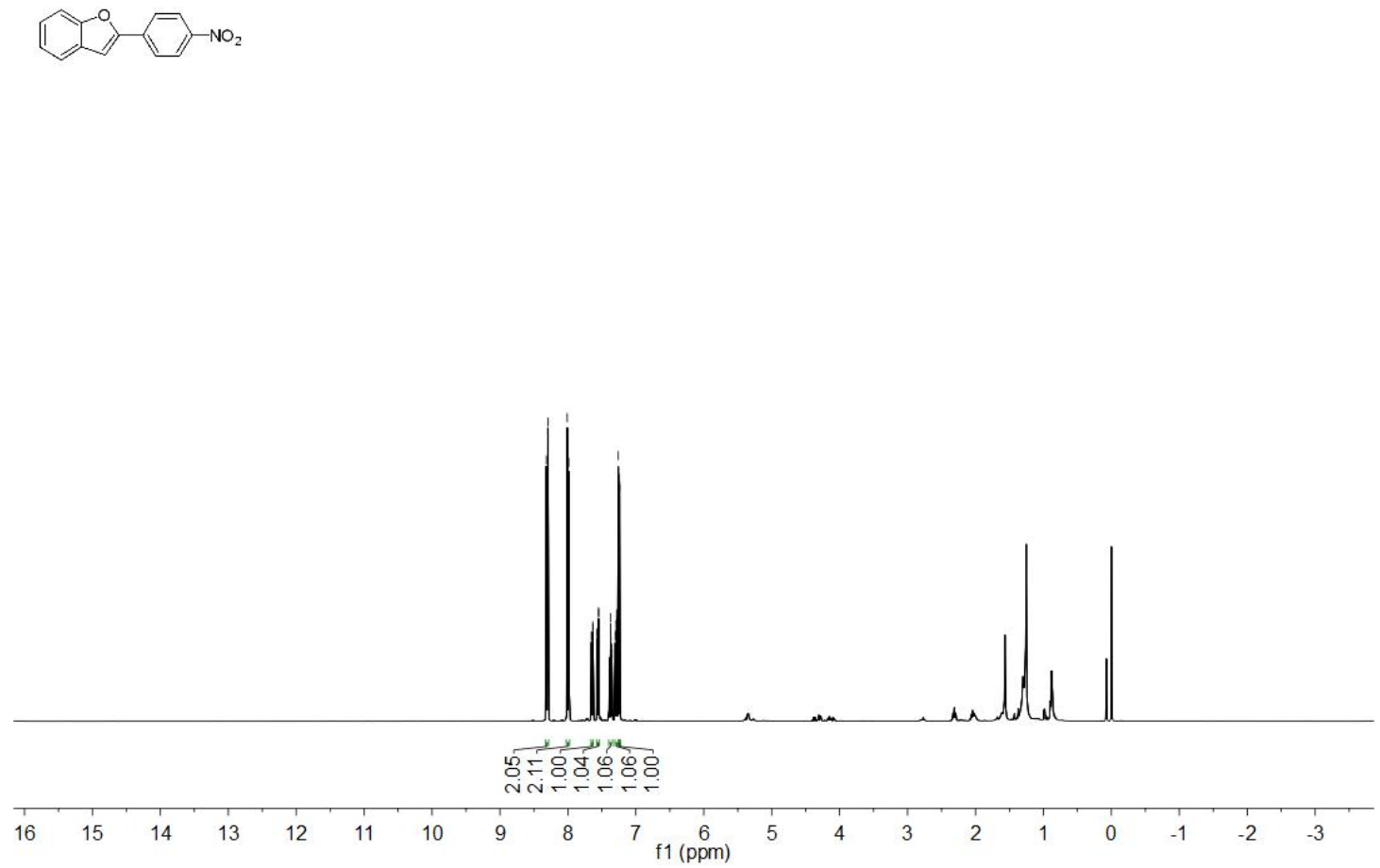

Figure S72. The ${ }^{13} \mathrm{C}$ NMR spectrums of 2-(4-nitrophenyl)benzofuran (3ea)
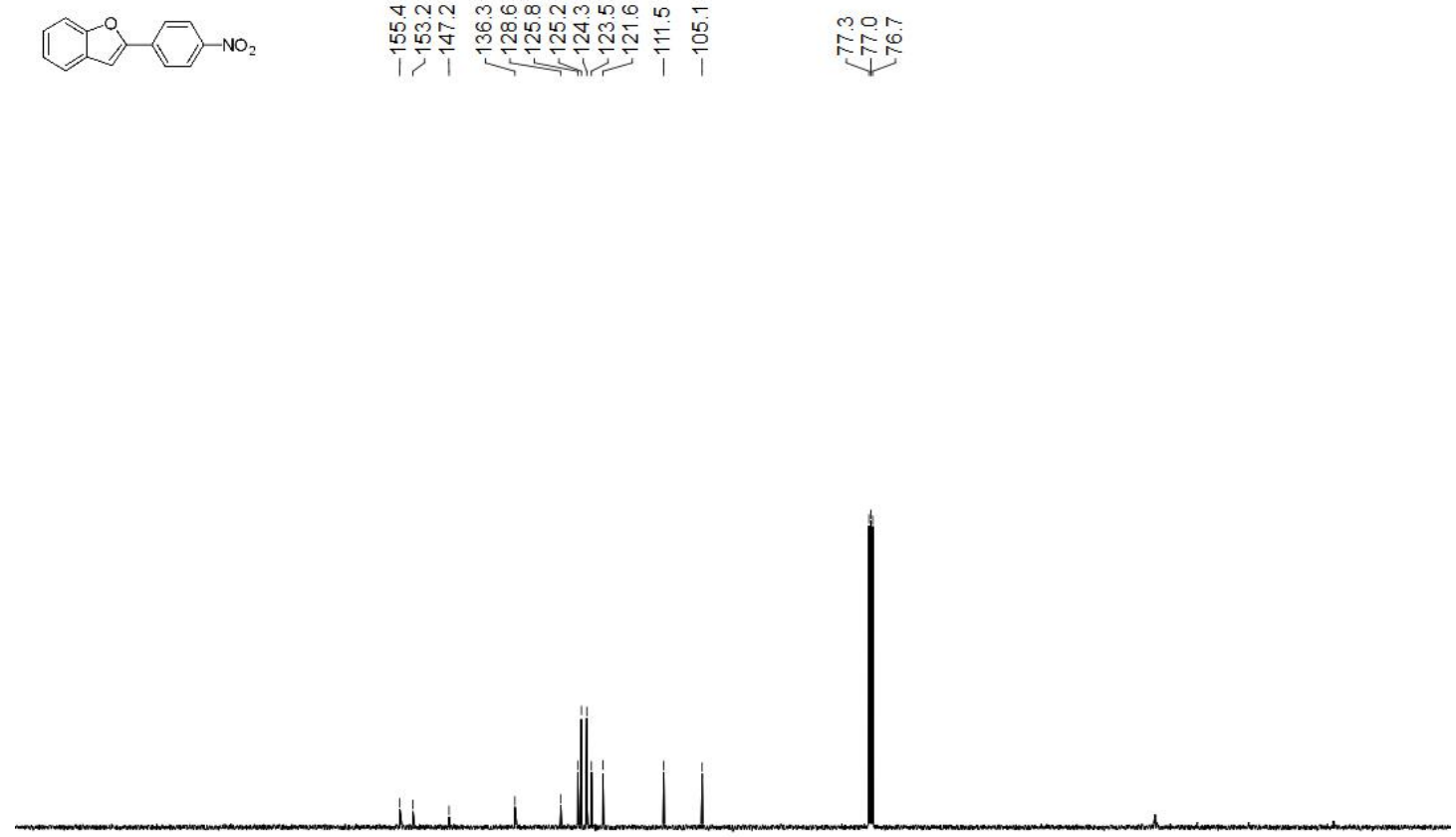

$\begin{array}{llllllllllllllllllllll}210 & 200 & 190 & 180 & 170 & 160 & 150 & 140 & 130 & 120 & 110 \begin{array}{c}100 \\ \mathrm{f} 1(\mathrm{ppm})\end{array} & 90 & 80 & 70 & 60 & 50 & 40 & 30 & 20 & 10 & 0 & -10\end{array}$ 
Figure S73. The ${ }^{1}$ H NMR spectrums of 1-(4-(benzofuran-2-yl)phenyl)ethanone (3ed)
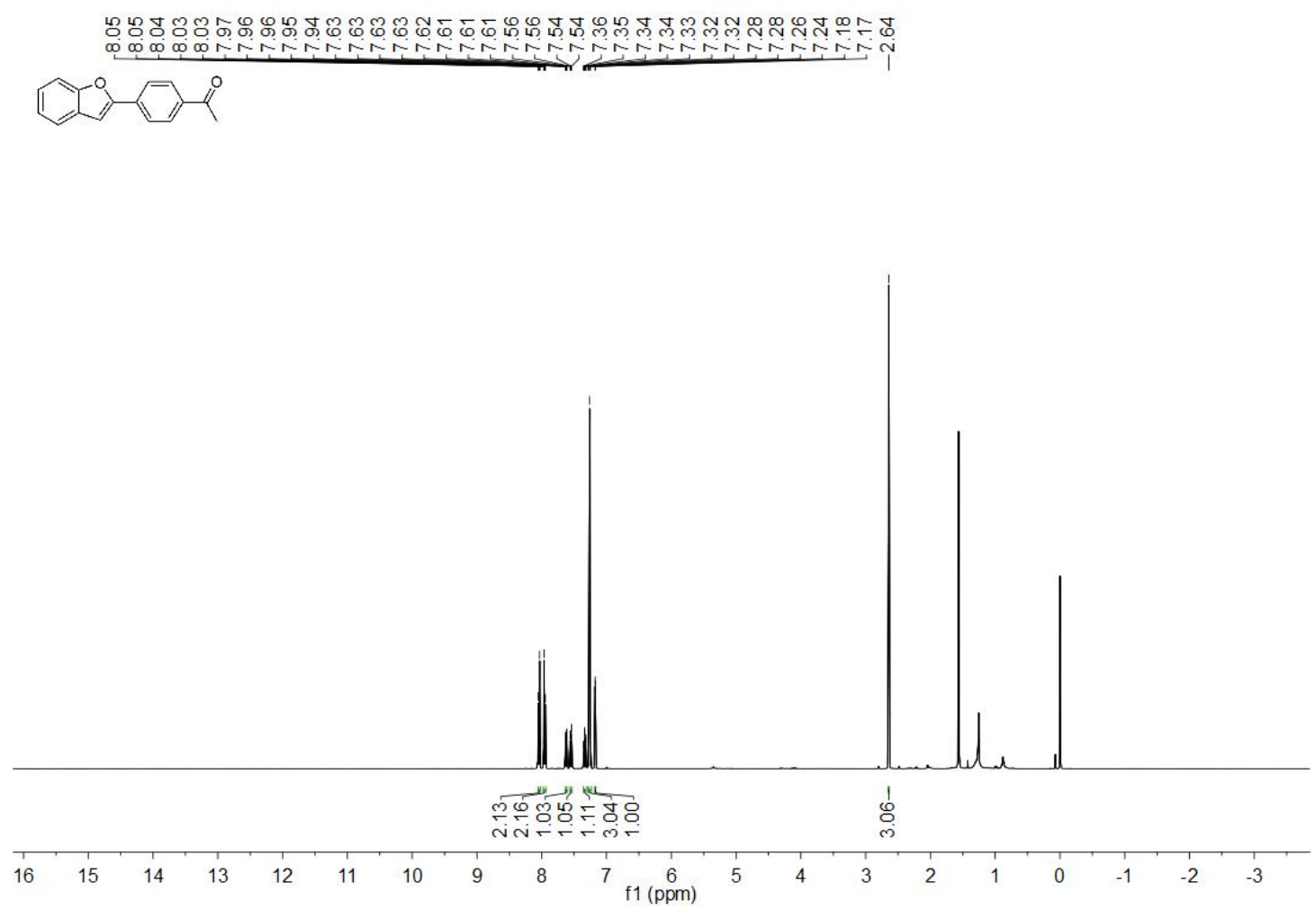

Figure S74. The ${ }^{13} \mathrm{C}$ NMR spectrums of 1-(4-(benzofuran-2-yl)phenyl)ethanone (3ed)

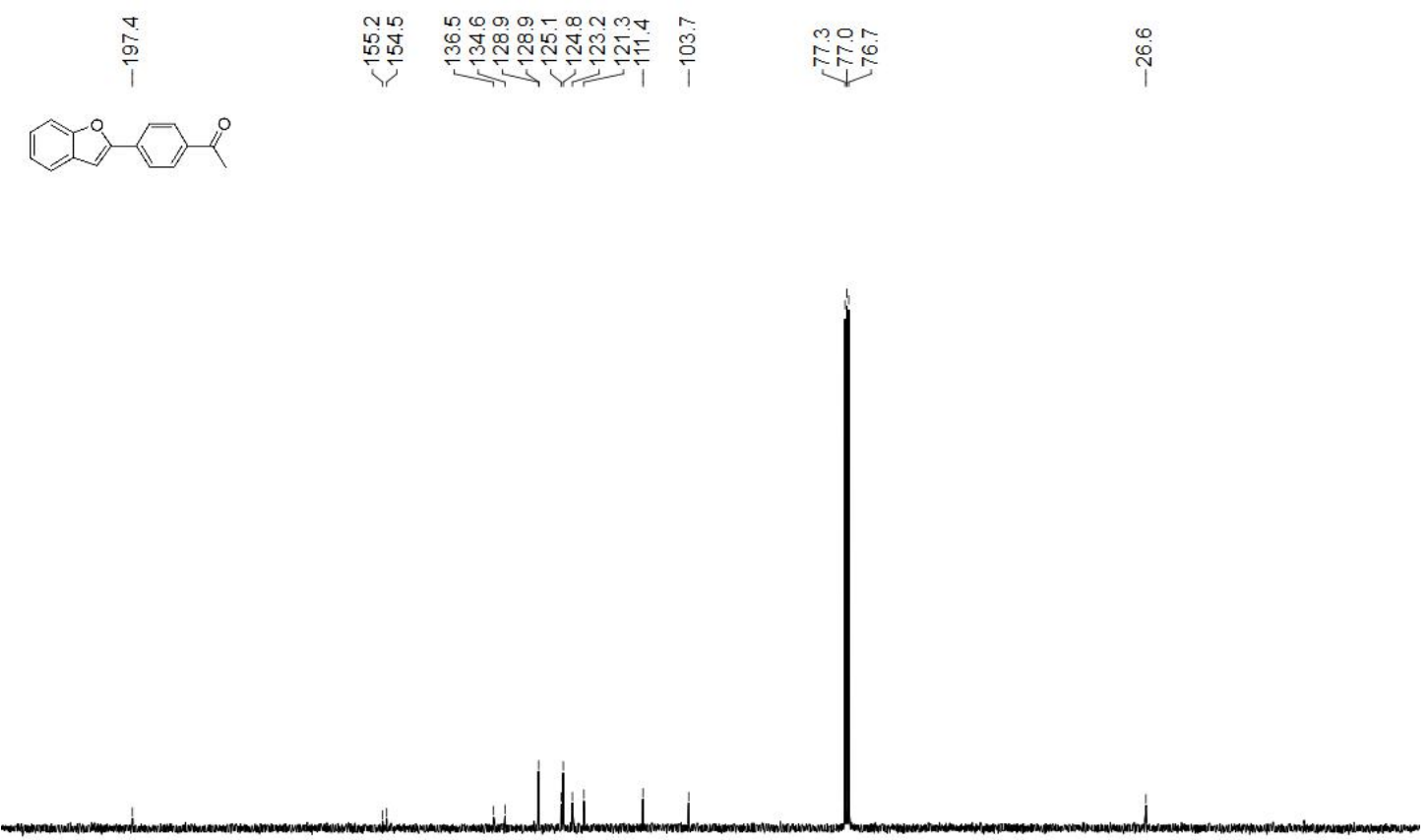

$\begin{array}{llllllllllllllllllllll}210 & 200 & 190 & 180 & 170 & 160 & 150 & 140 & 130 & 120 & 110 \begin{array}{c}100 \\ \mathrm{f} 1(\mathrm{ppm})\end{array} & 90 & 80 & 70 & 60 & 50 & 40 & 30 & 20 & 10 & 0 & -10\end{array}$ 
Figure S75. The ${ }^{1}$ H NMR spectrums of 4-(5-ethylthiophen-2-yl)benzaldehyde (5ac)

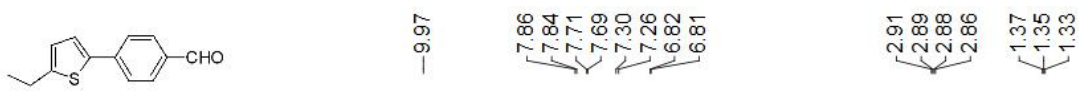

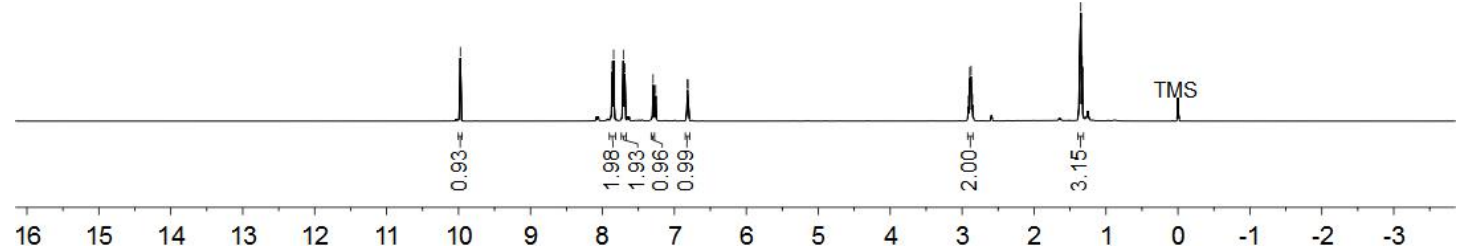

Figure S76. The ${ }^{13} \mathrm{C}$ NMR spectrums of 4-(5-ethylthiophen-2-yl)benzaldehyde (5ac)
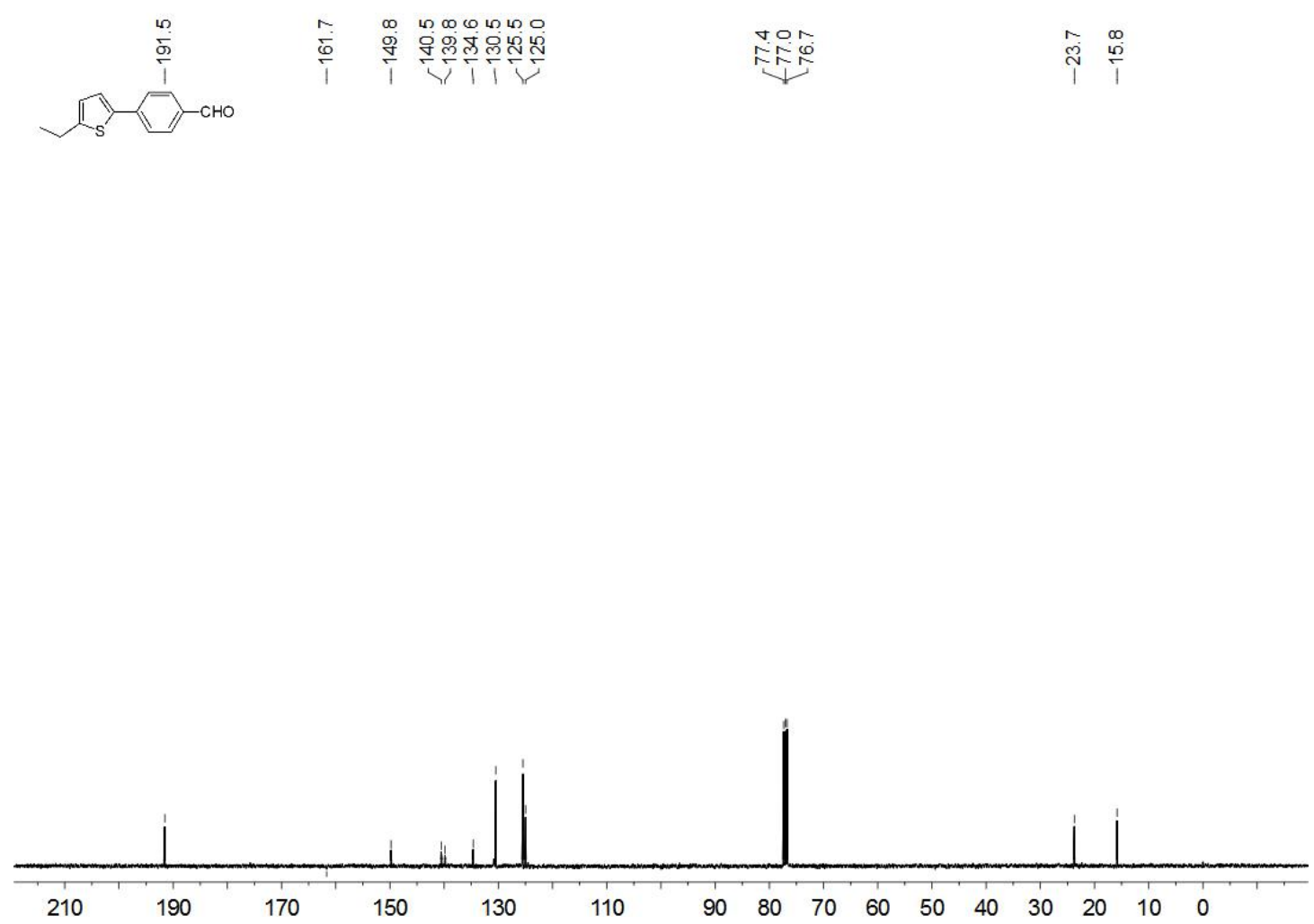
Figure S77. The ${ }^{1} \mathrm{H}$ NMR spectrums of 3-(5-ethylthiophen-2-yl)pyridine (5aj)
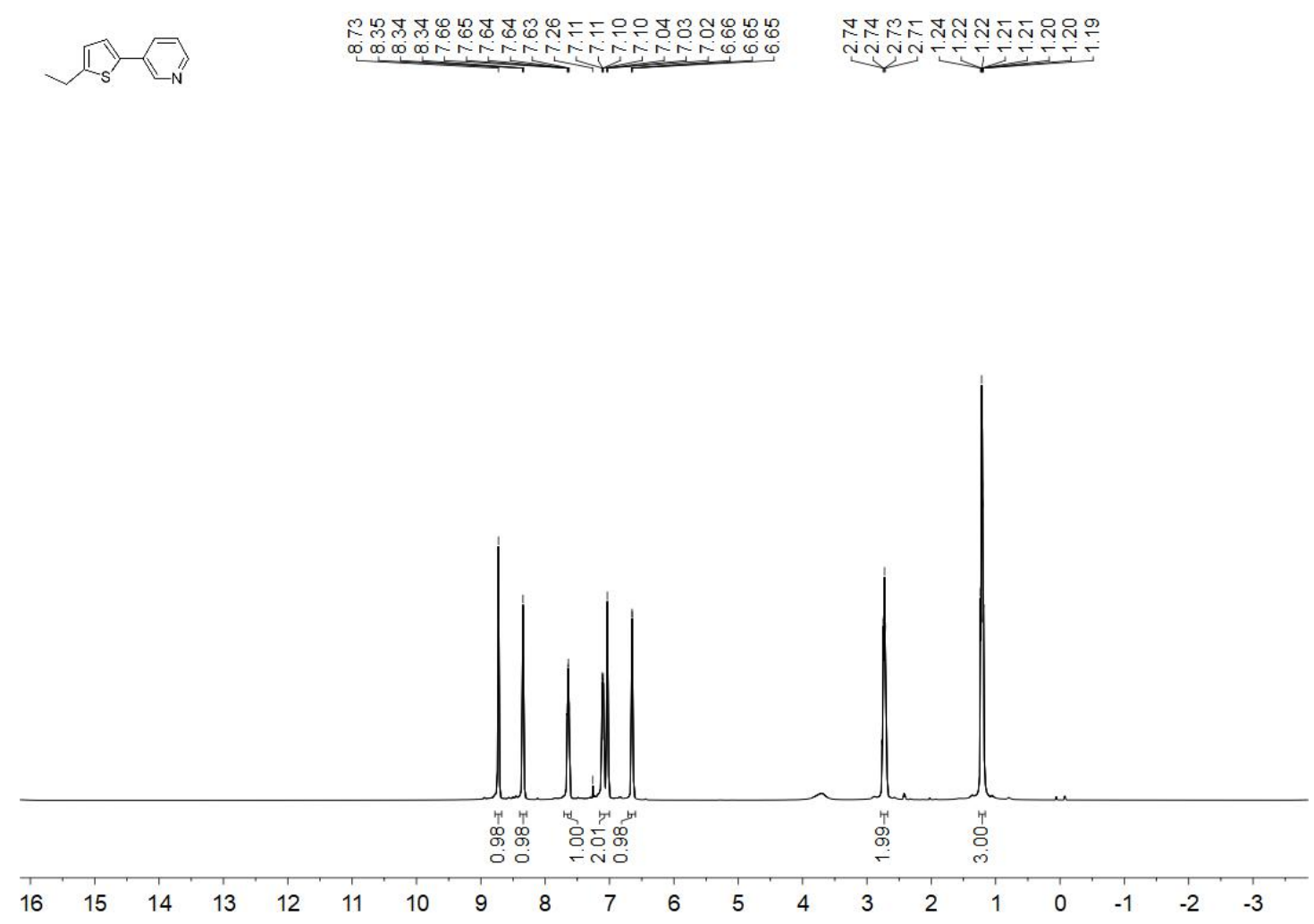

Figure S78. The ${ }^{13} \mathrm{C}$ NMR spectrums of 3-(5-ethylthiophen-2-yl)pyridine (5aj)
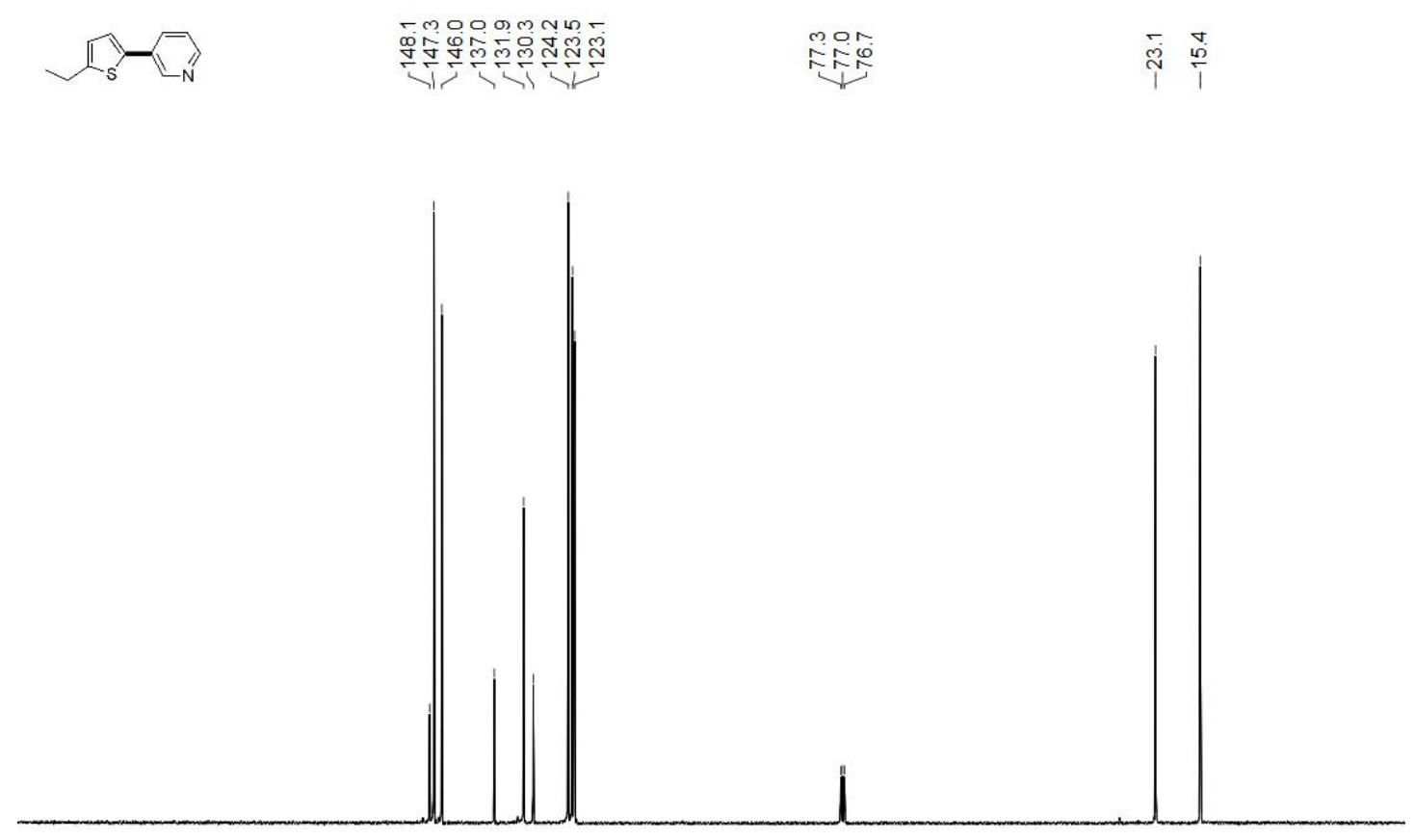

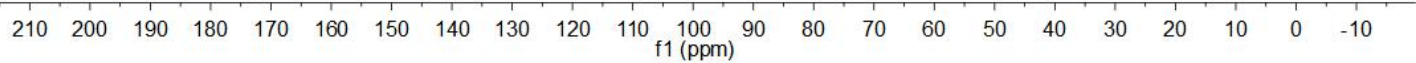


Figure S79. The ${ }^{1}$ H NMR spectrums of 4-(5-ethylthiophen-2-yl)isoquinoline (5ak)
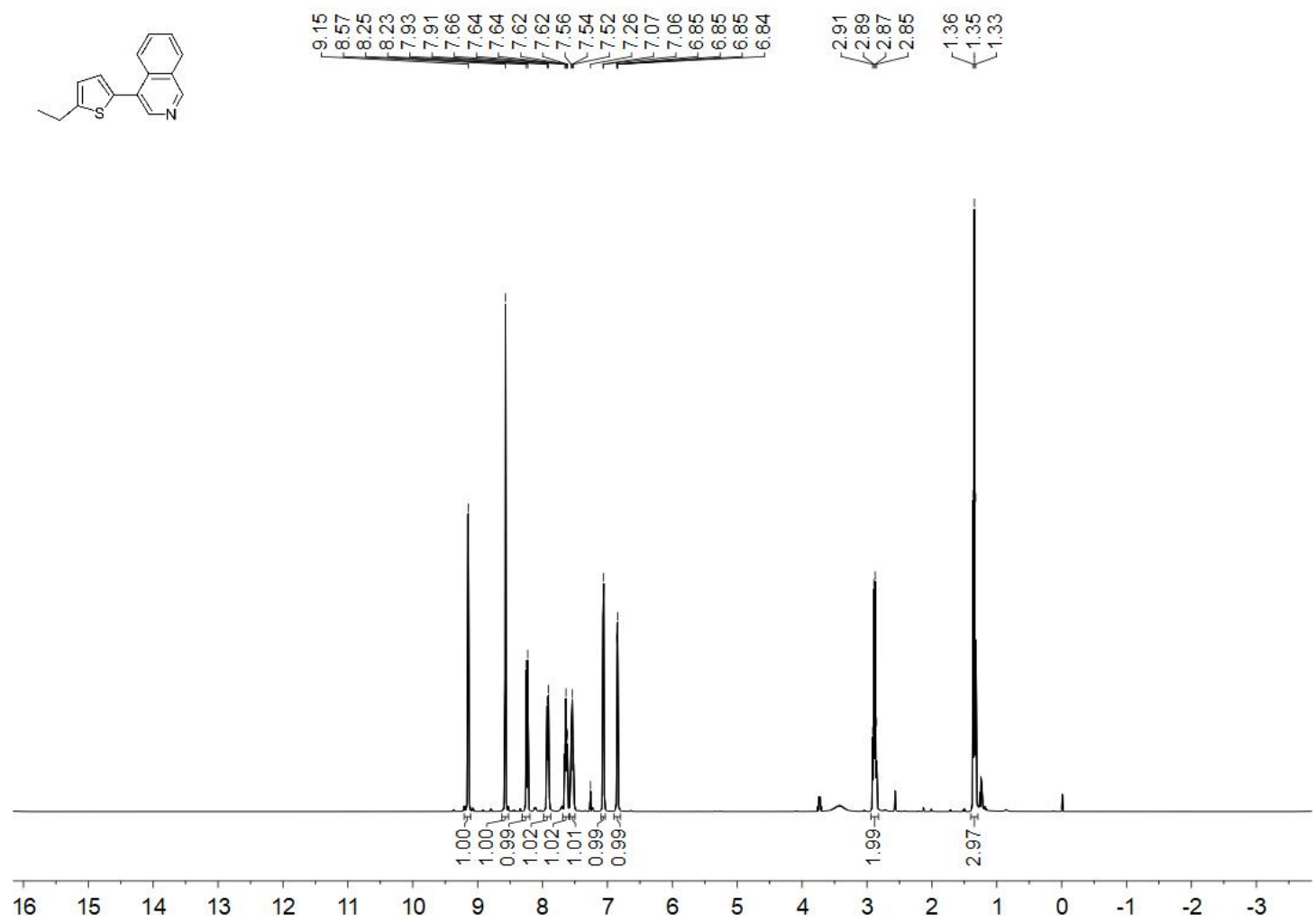

Figure S80. The ${ }^{13} \mathrm{C}$ NMR spectrums of 4-(5-ethylthiophen-2-yl)isoquinoline (5ak)

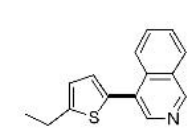

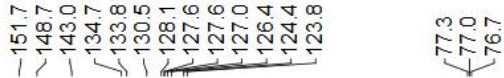

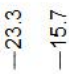

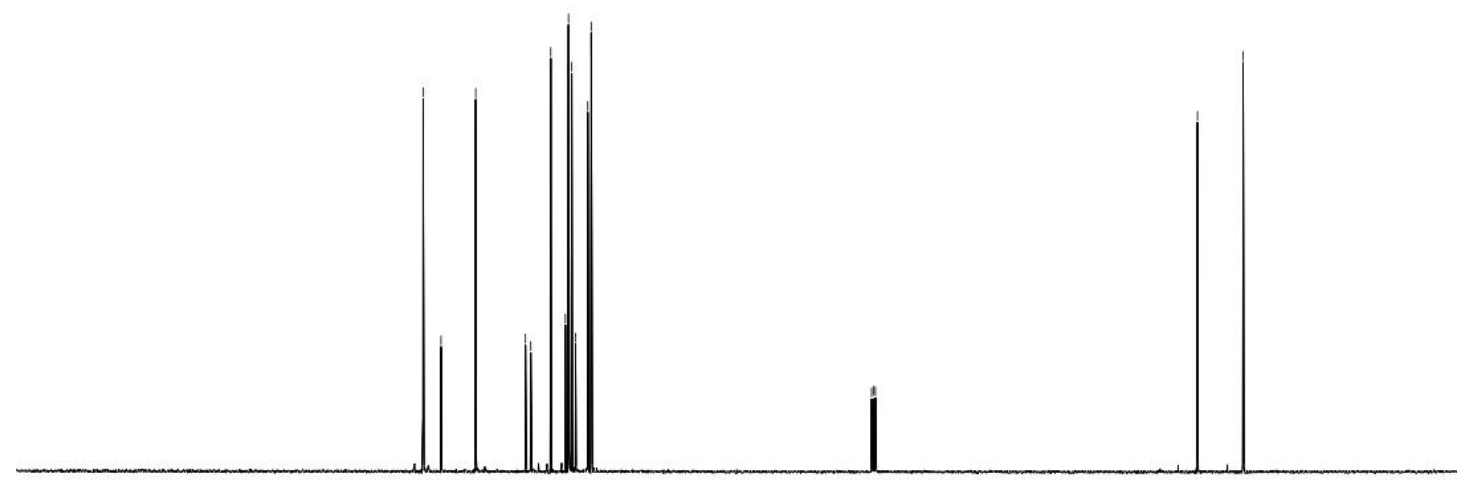

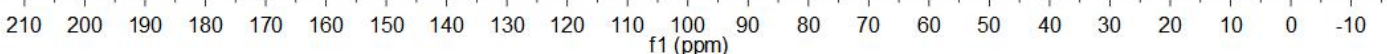


Figure S81. The ${ }^{1} \mathrm{H}$ NMR spectrums of 1-(4-(2,4-dimethylthiazol-5-yl)phenyl)ethanone (5bd)

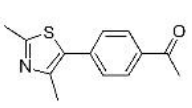

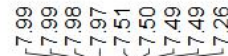

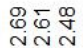

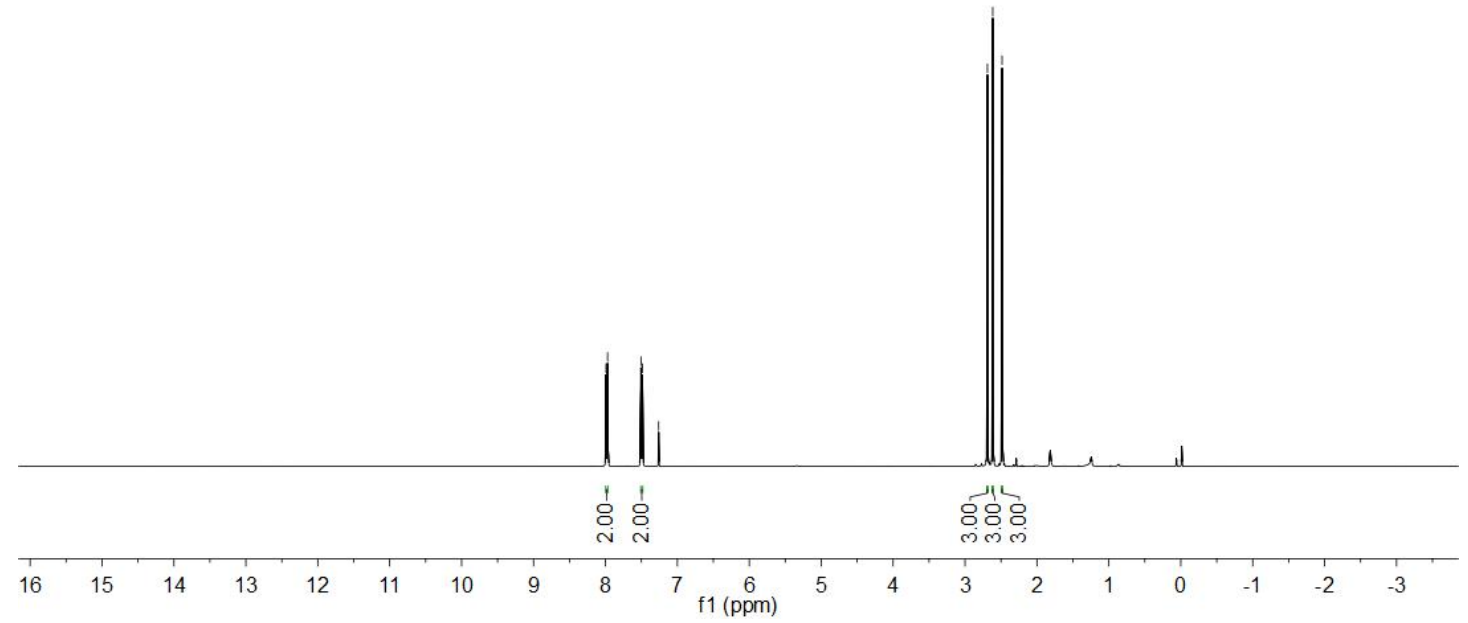

Figure S82. The ${ }^{13} \mathrm{C}$ NMR spectrums of 1-(4-(2,4-dimethylthiazol-5-yl)phenyl)ethanone (5bd)
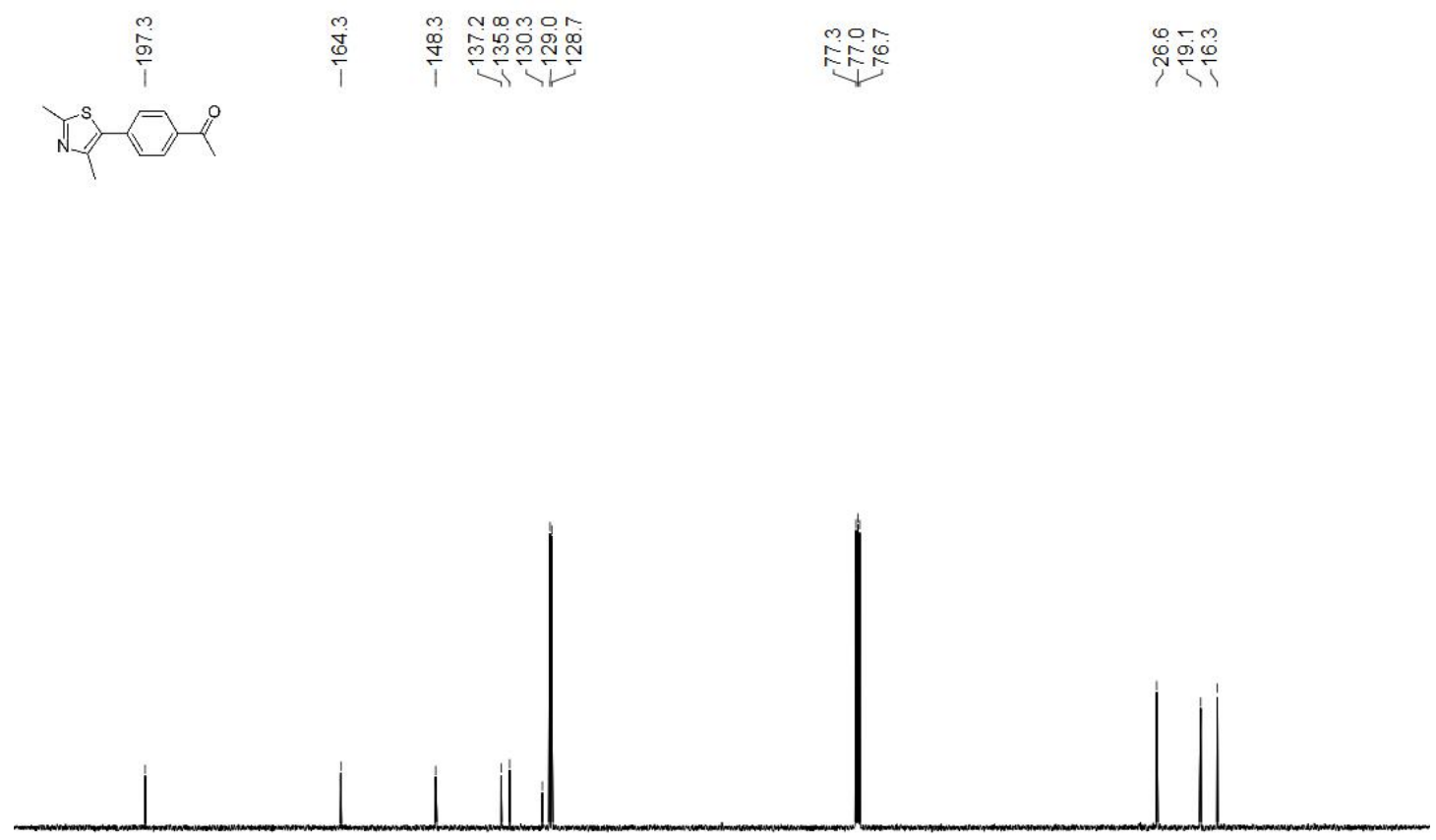

$\begin{array}{llllllllllllllllllllll}210 & 200 & 190 & 180 & 170 & 160 & 150 & 140 & 130 & 120 & 110 \begin{array}{l}100 \\ \mathrm{f} 1(\mathrm{ppm})\end{array} & 90 & 80 & 70 & 60 & 50 & 40 & 30 & 20 & 10 & 0 & -10\end{array}$ 
Figure S83. The ${ }^{1} \mathrm{H}$ NMR spectrums of 2,4-dimethyl-5-phenylthiazole (5bf)
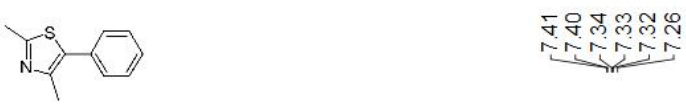

$\stackrel{\substack{i \\ \stackrel{\infty}{i}}}{\infty}$

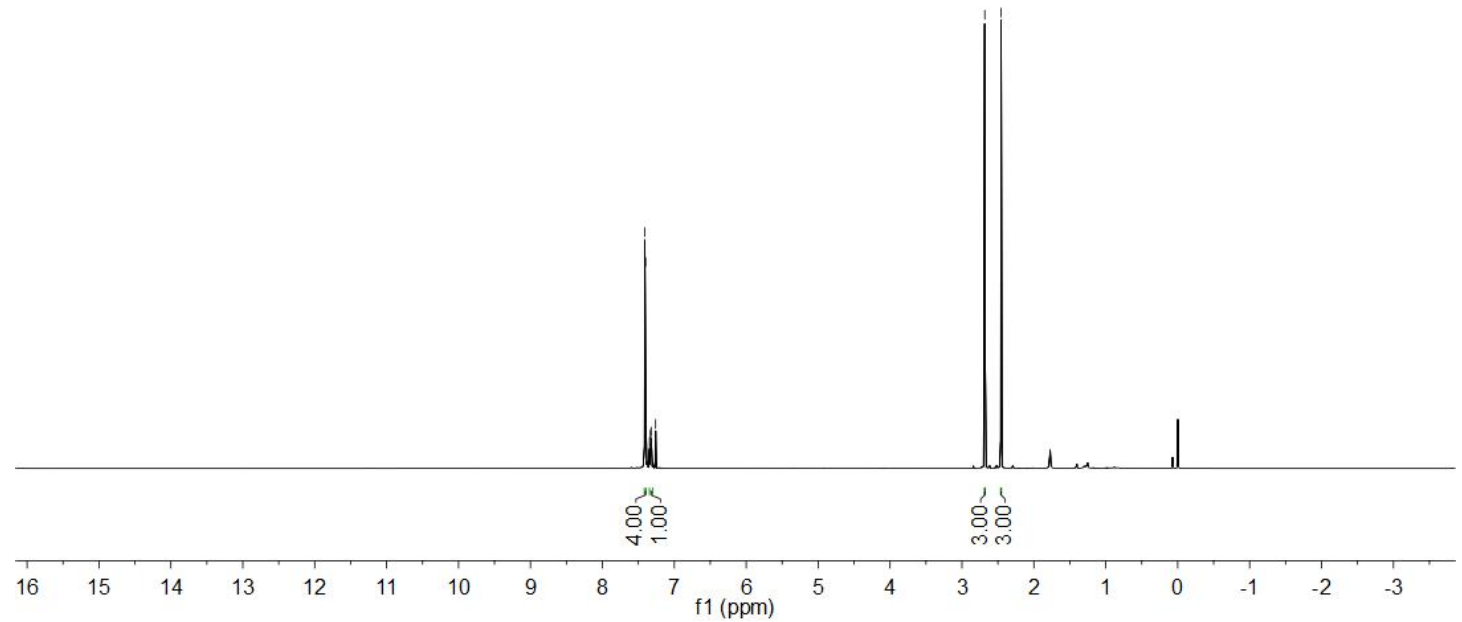

Figure S84. The ${ }^{13} \mathrm{C}$ NMR spectrums of 2,4-dimethyl-5-phenylthiazole (5bf)

N-j|l

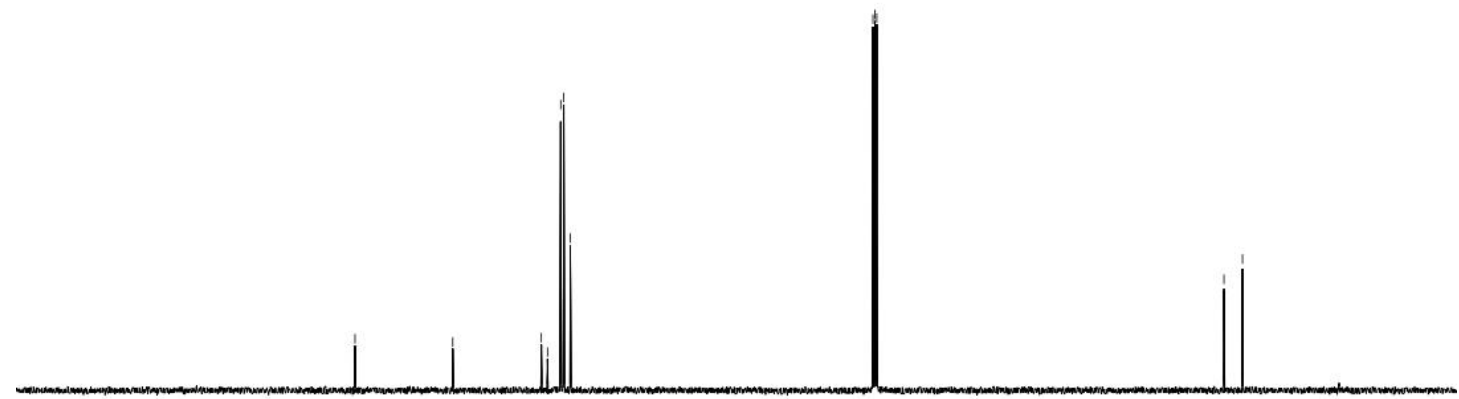

$\begin{array}{lllllllllllllllllllllll}210 & 200 & 190 & 180 & 170 & 160 & 150 & 140 & 130 & 120 & 110 & 100 & 100 & 80 & 70 & 60 & 50 & 40 & 30 & 20 & 10 & 0 & -10\end{array}$ 
Figure S85. The ${ }^{1} \mathrm{H}$ NMR spectrums of 2,4-dimethyl-5-(naphthalen-1-yl)thiazole (5bm)

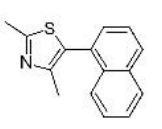

б.

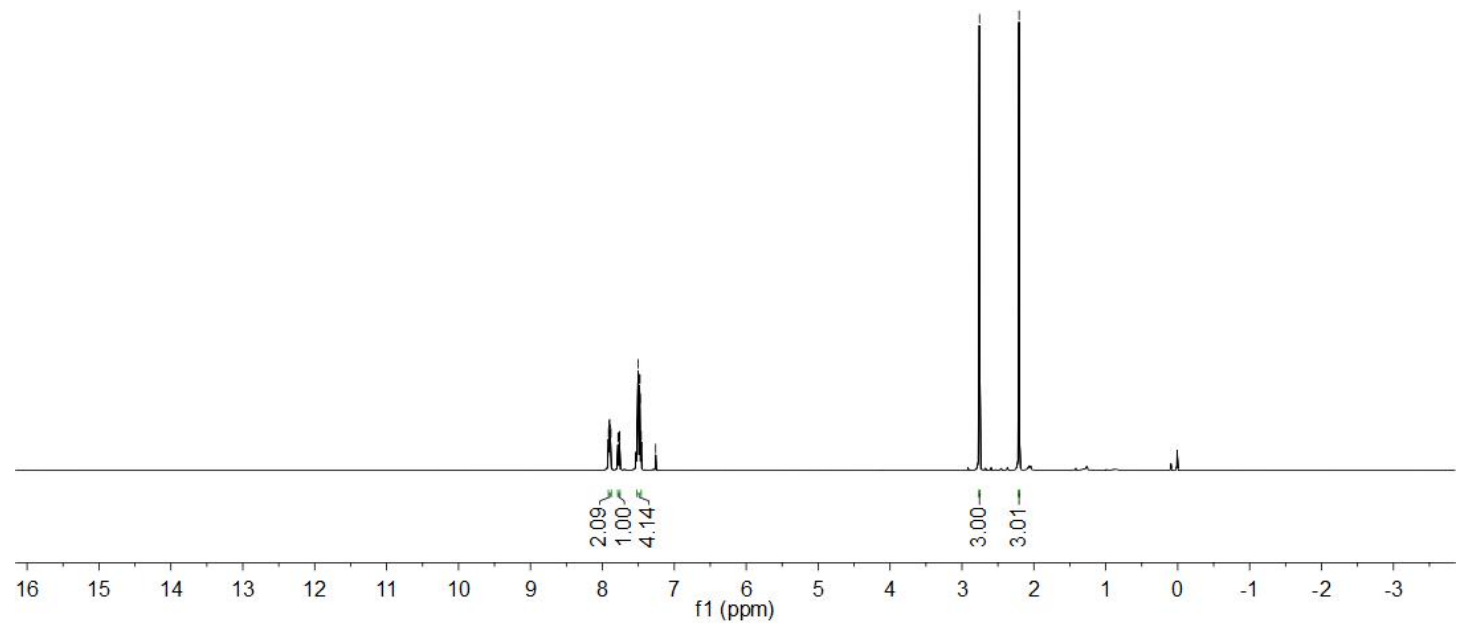

Figure S86. The ${ }^{13} \mathrm{C}$ NMR spectrums of 2,4-dimethyl-5-(naphthalen-1-yl)thiazole (5bm)
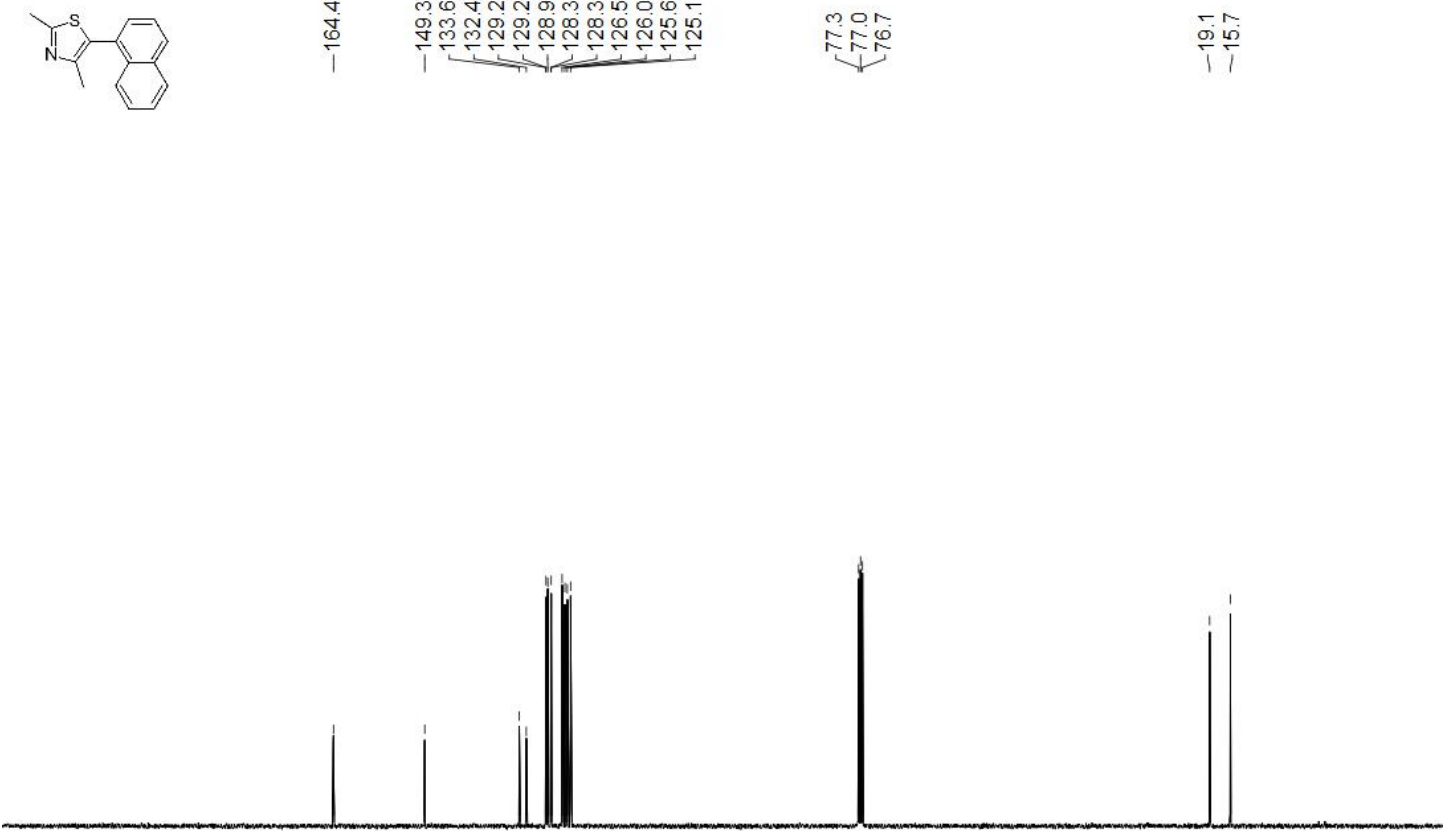

$\begin{array}{lllllllllllllllllllllll}210 & 200 & 190 & 180 & 170 & 160 & 150 & 140 & 130 & 120 & 110 \begin{array}{c}100 \\ \mathrm{f} 1(\mathrm{ppm})\end{array} & 90 & 80 & 70 & 60 & 50 & 40 & 30 & 20 & 10 & 0 & -10\end{array}$ 
Figure S87. The ${ }^{1}$ H NMR spectrums of 2,4-dimethyl-5-(2-methylnaphthalen-1-yl)thiazole (5bn)

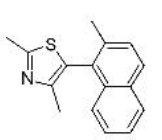

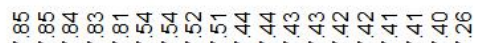

iㅗㅇㅛ

列

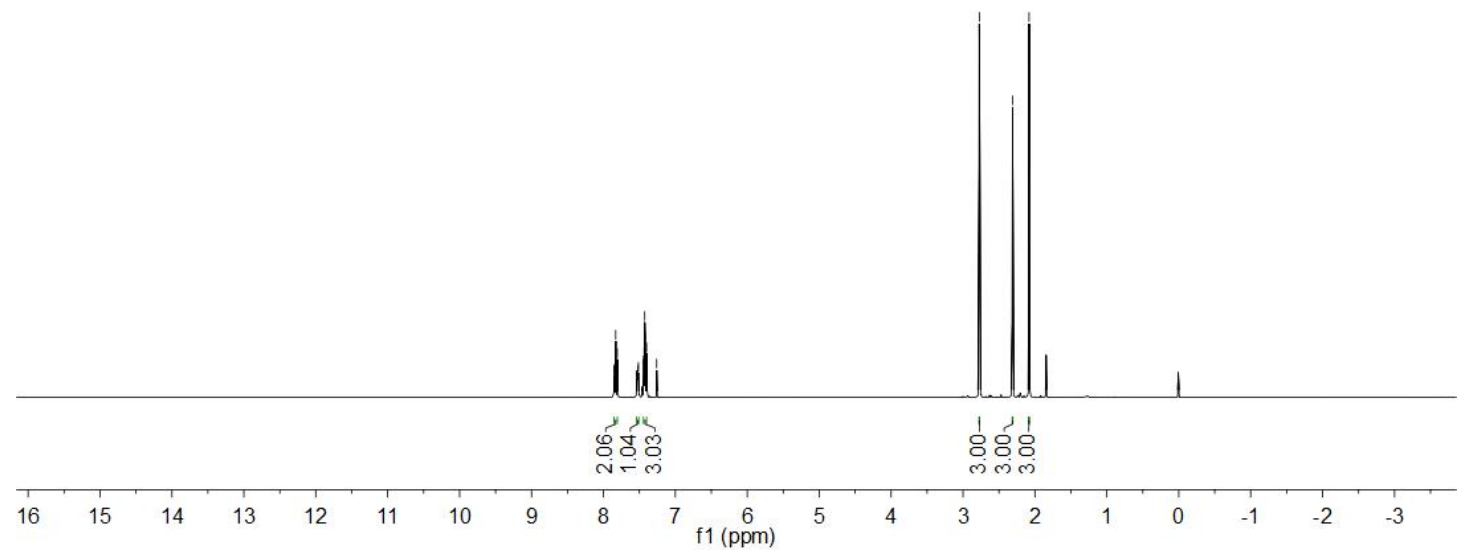

Figure S88. The ${ }^{13} \mathrm{C}$ NMR spectrums of 2,4-dimethyl-5-(2-methylnaphthalen-1-yl)thiazole (5bn)
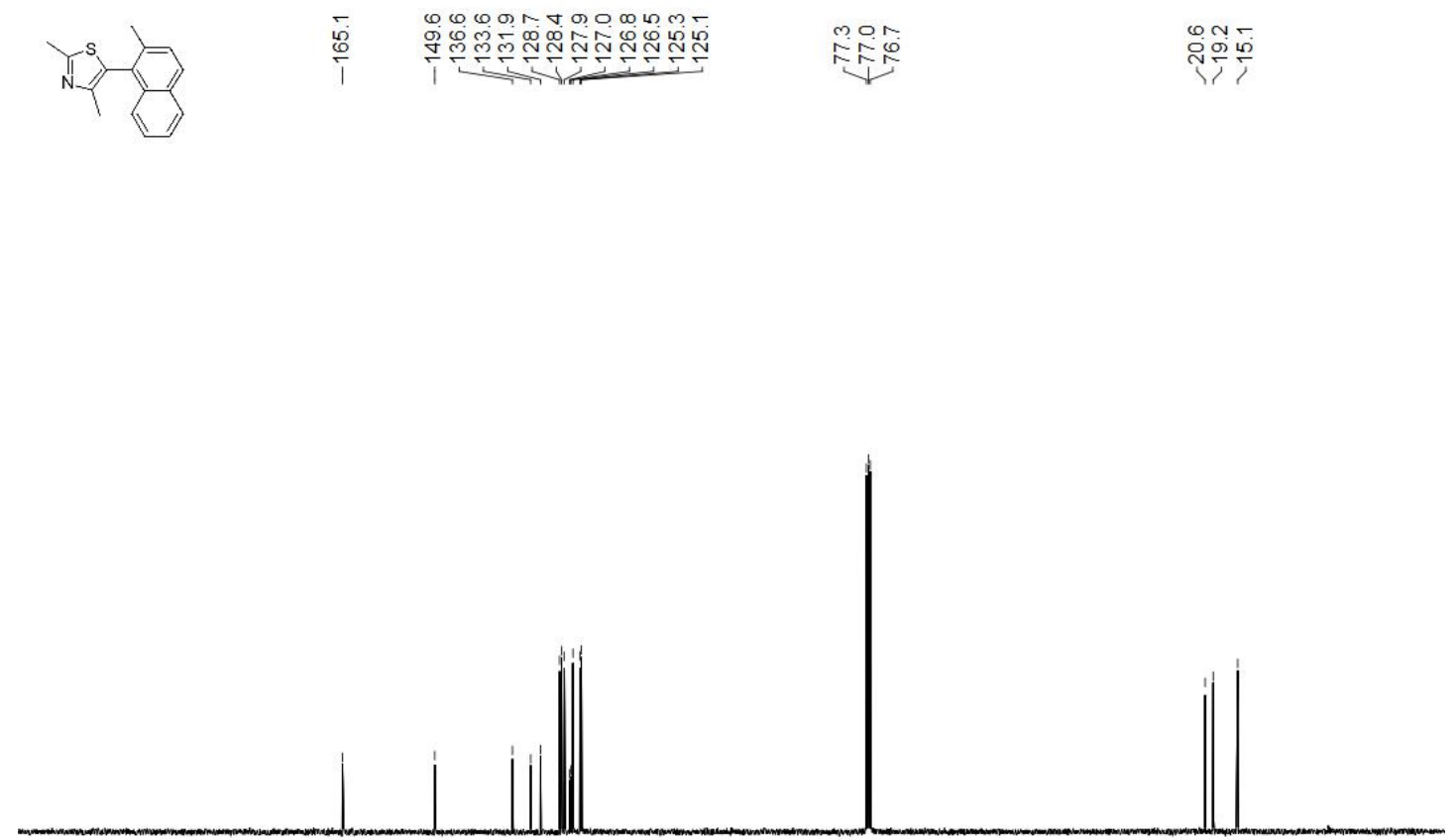

$\begin{array}{lllllllllllllllllllllll}210 & 200 & 190 & 180 & 170 & 160 & 150 & 140 & 130 & 120 & 110 & 100 & 90 & 80 & 70 & 60 & 50 & 40 & 30 & 20 & 10 & 0 & -10\end{array}$ 
Figure S89. The ${ }^{1} \mathrm{H}$ NMR spectrums of methyl 4-(2,4-dimethylthiazol-5-yl)benzoate (5bt)

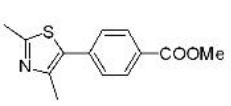

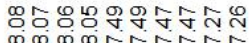

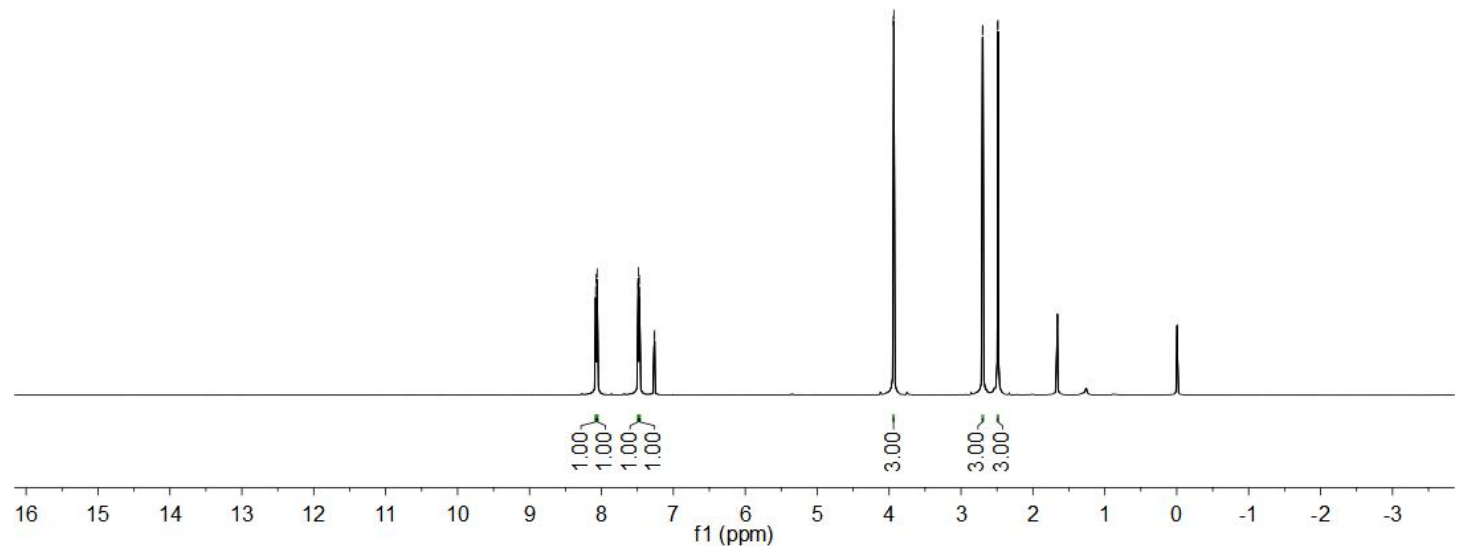

Figure S90. The ${ }^{13} \mathrm{C}$ NMR spectrums of methyl 4-(2,4-dimethylthiazol-5-yl)benzoate (5bt)
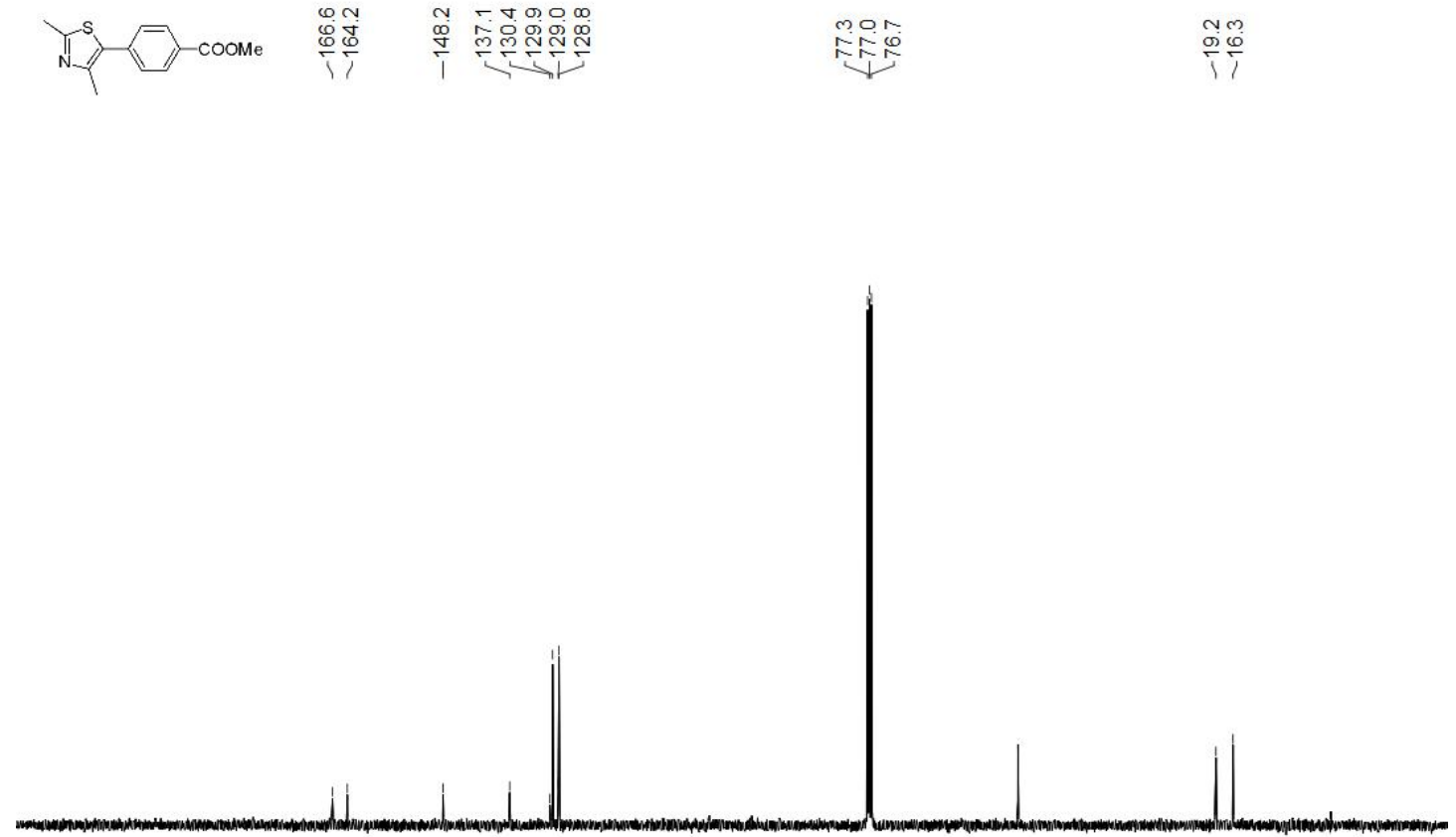

$\begin{array}{lllllllllllllllllllllll}210 & 200 & 190 & 180 & 170 & 160 & 150 & 140 & 130 & 120 & 110 \begin{array}{c}100 \\ \mathrm{f} 1(\mathrm{ppm})\end{array} & 90 & 80 & 70 & 60 & 50 & 40 & 30 & 20 & 10 & 0 & -10\end{array}$ 
Figure S91. The ${ }^{1} \mathrm{H}$ NMR spectrums of 2,4,5-triphenyloxazole (5cf)

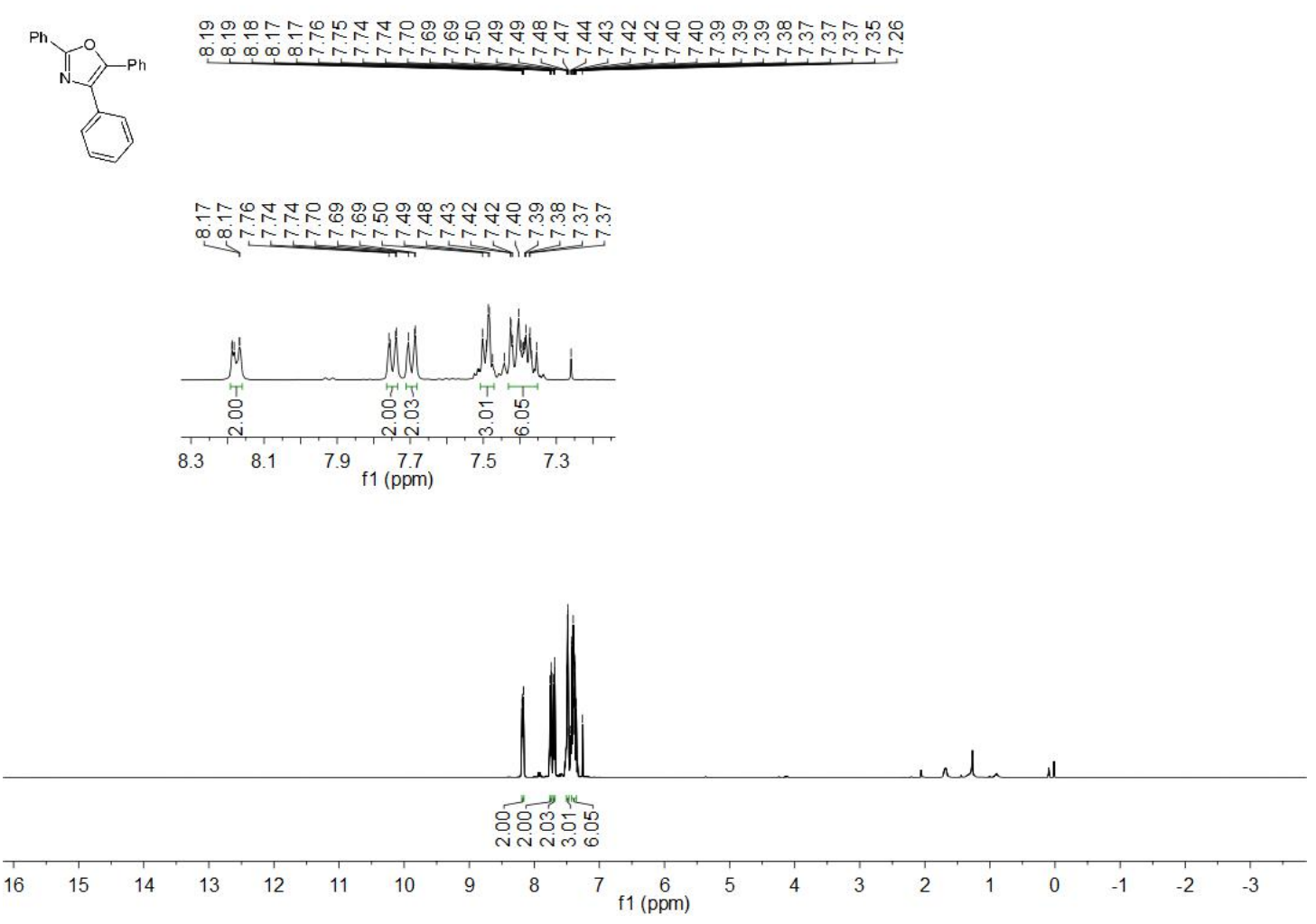

Figure S92. The ${ }^{13} \mathrm{C}$ NMR spectrums of 2,4,5-triphenyloxazole (5cf)
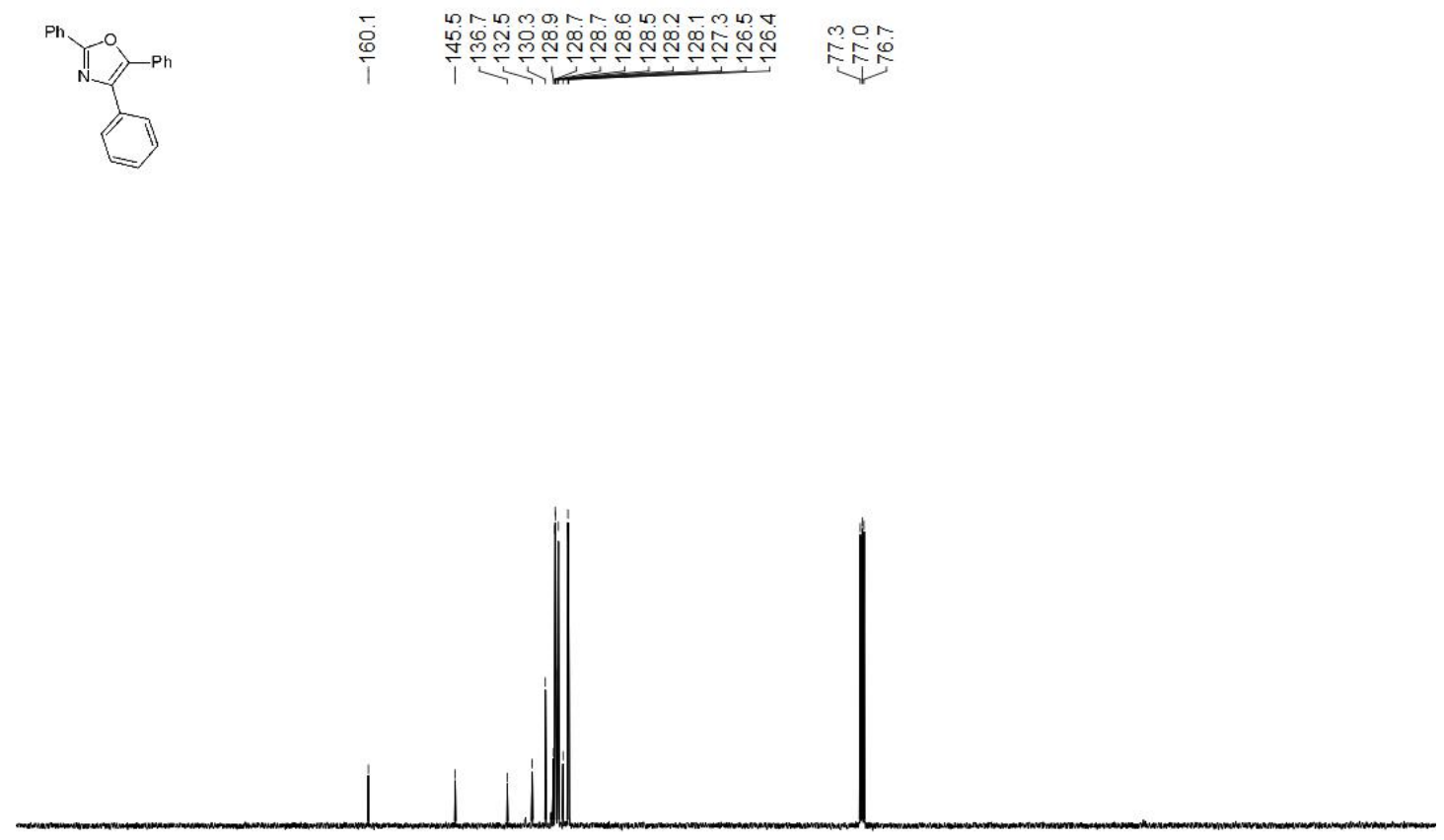

$\begin{array}{lllllllllllllllllllllllll}210 & 200 & 190 & 180 & 170 & 160 & 150 & 140 & 130 & 120 & 110 & 100 & 90 & 80 & 70 & 60 & 50 & 40 & 30 & 20 & 10 & 0 & -10\end{array}$ 
Figure S93. The ${ }^{1} \mathrm{H}$ NMR spectrums of 4-(6-chloropyridin-3-yl)-2,5-diphenyloxazole (5cp)

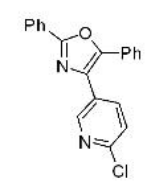

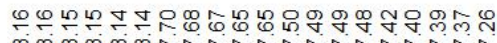

$\infty 0000000$

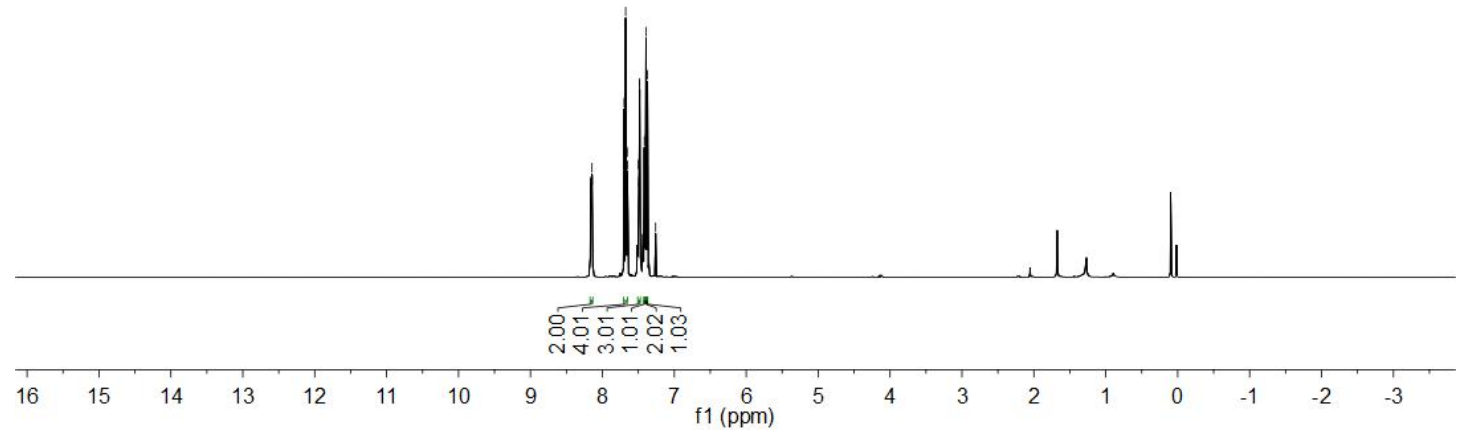

Figure S94. The ${ }^{13} \mathrm{C}$ NMR spectrums of 4-(6-chloropyridin-3-yl)-2,5-diphenyloxazole (5cp)<smiles>Clc1ccc(-c2nc(-c3ccccc3)oc2-c2ccccc2)cn1</smiles>

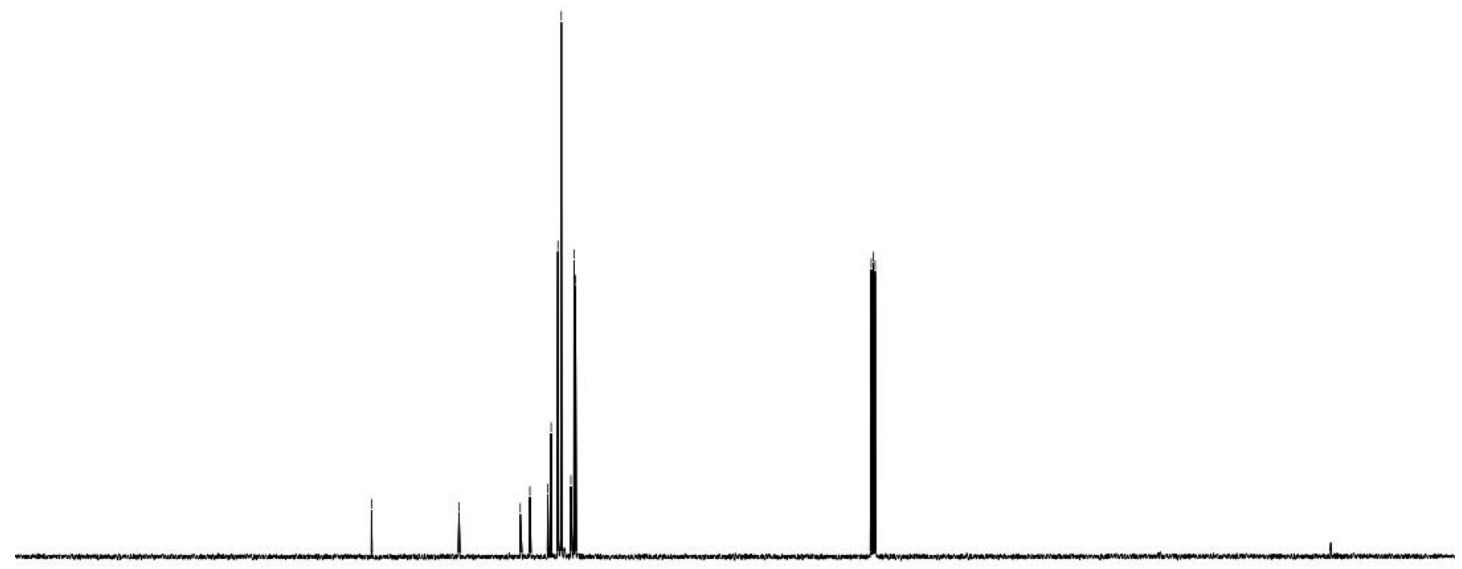

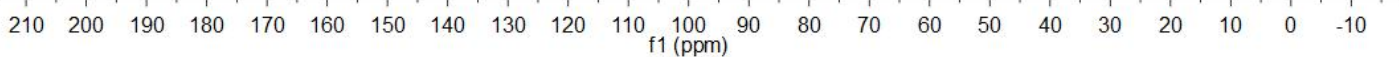


Figure S95. The ${ }^{1} \mathrm{H}$ NMR spectrums of 2,5-diphenyl-4-(p-tolyl)oxazole (5cu)
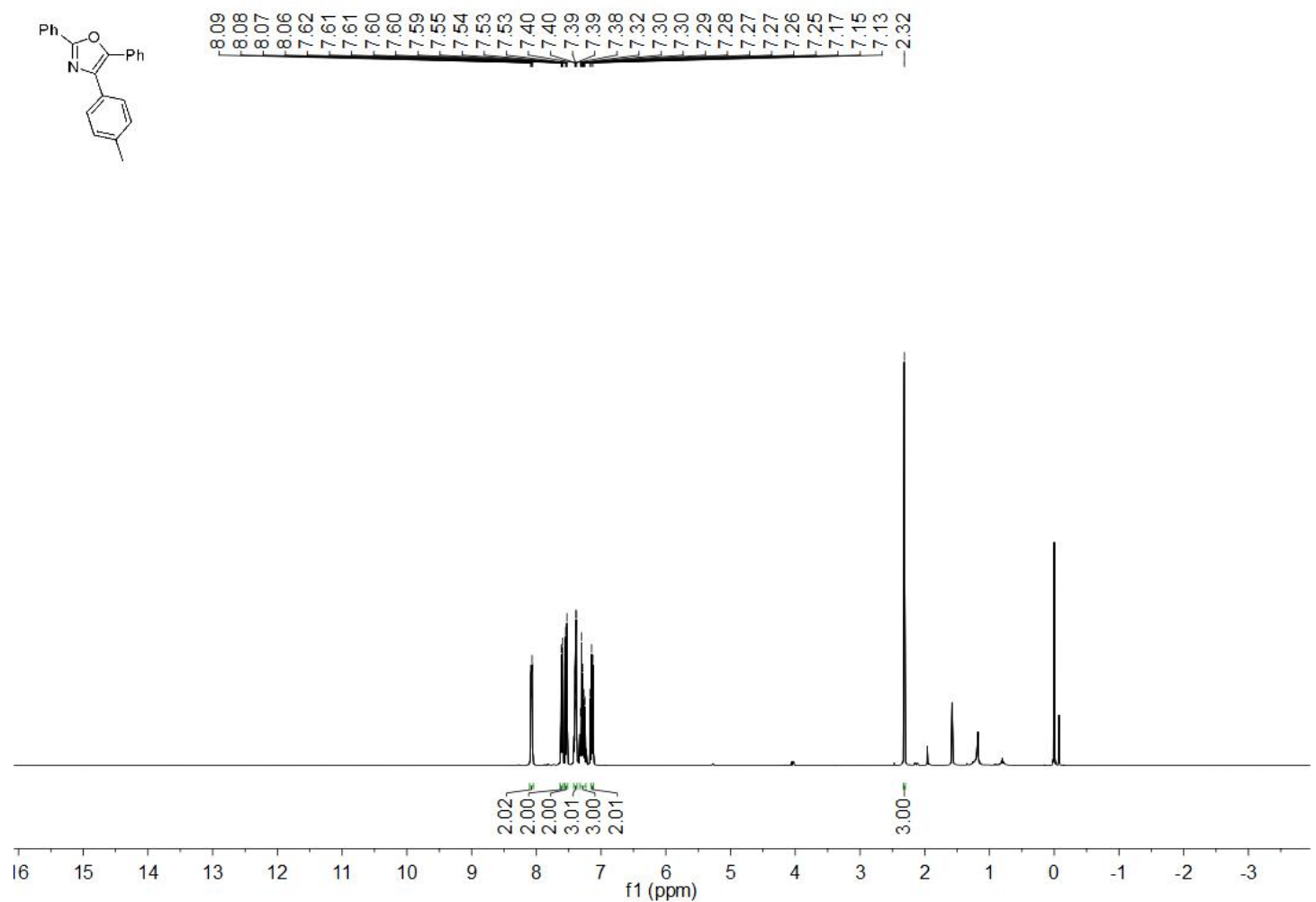

Figure S96. The ${ }^{13} \mathrm{C}$ NMR spectrums of 2,5-diphenyl-4-(p-tolyl)oxazole (5cu)
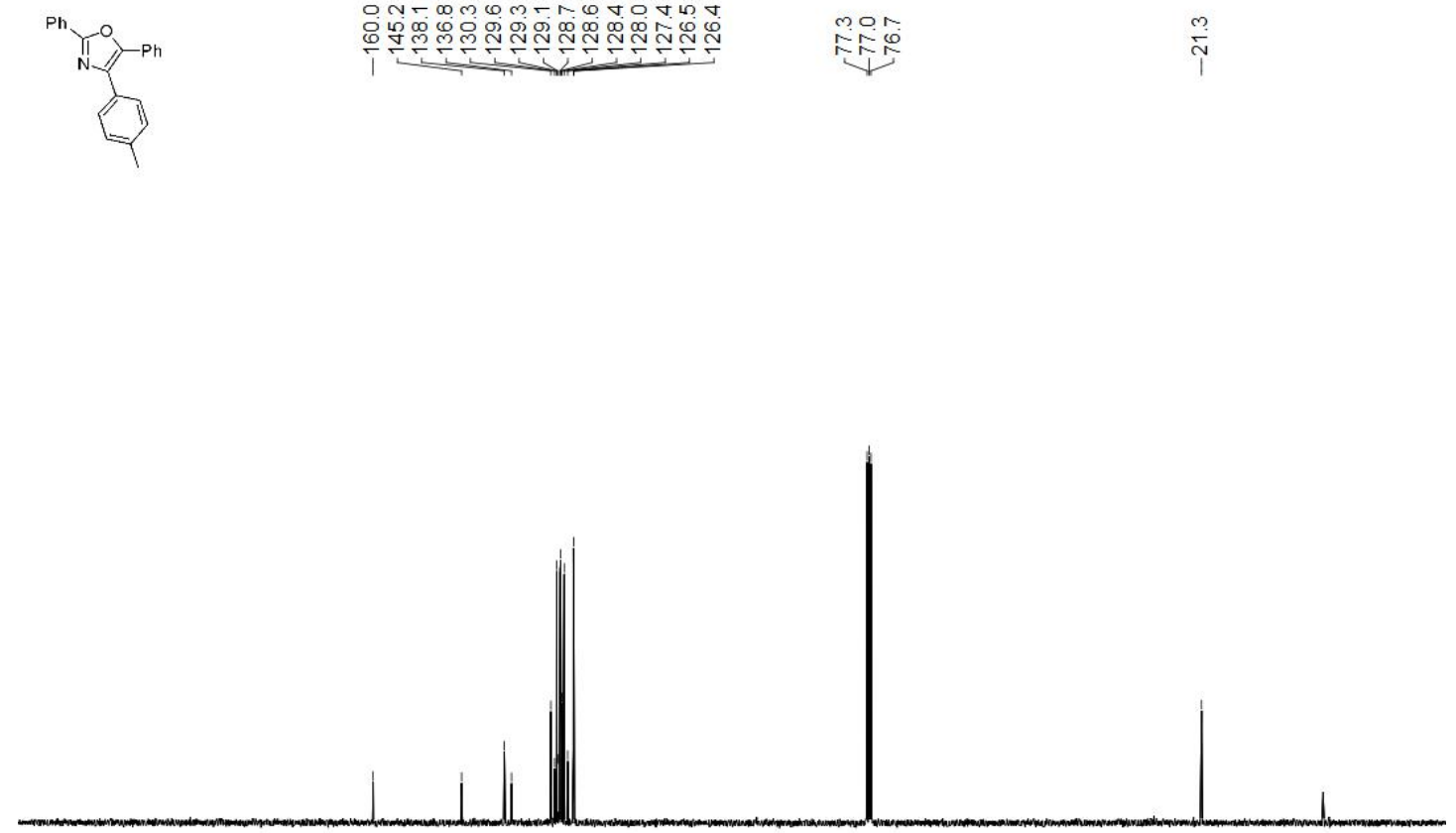

$\begin{array}{lllllllllllllllllllllll}210 & 200 & 190 & 180 & 170 & 160 & 150 & 140 & 130 & 120 & 110 \begin{array}{l}100 \\ \mathrm{f} 1(\mathrm{ppm})\end{array} & 90 & 80 & 70 & 60 & 50 & 40 & 30 & 20 & 10 & 0 & -10\end{array}$ 
Figure S97. The ${ }^{1}$ H NMR spectrums of 4-(4-chlorophenyl)-3,5-dimethylisoxazole (5de)

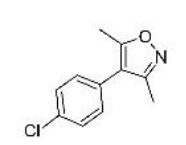

$$
\sqrt{\infty}
$$

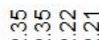

nูing

$\mathrm{Cl}$

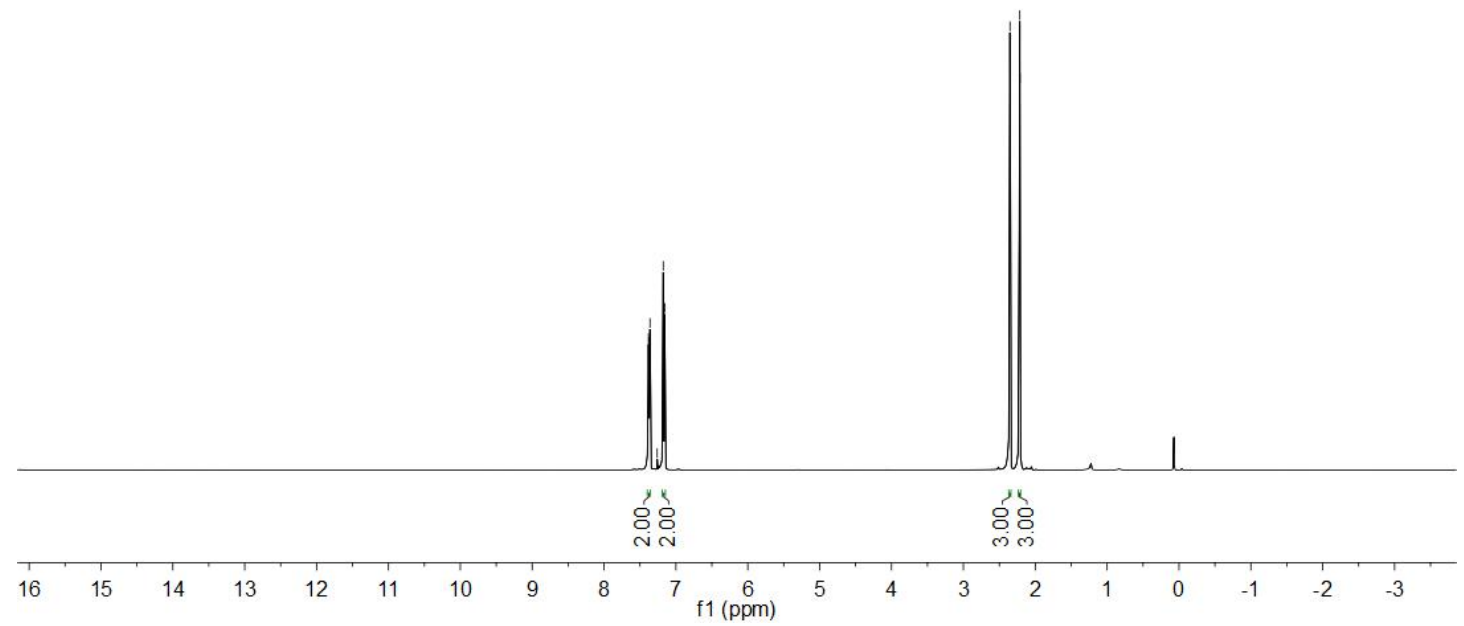

Figure S98. The ${ }^{13} \mathrm{C}$ NMR spectrums of 4-(4-chlorophenyl)-3,5-dimethylisoxazole (5de)
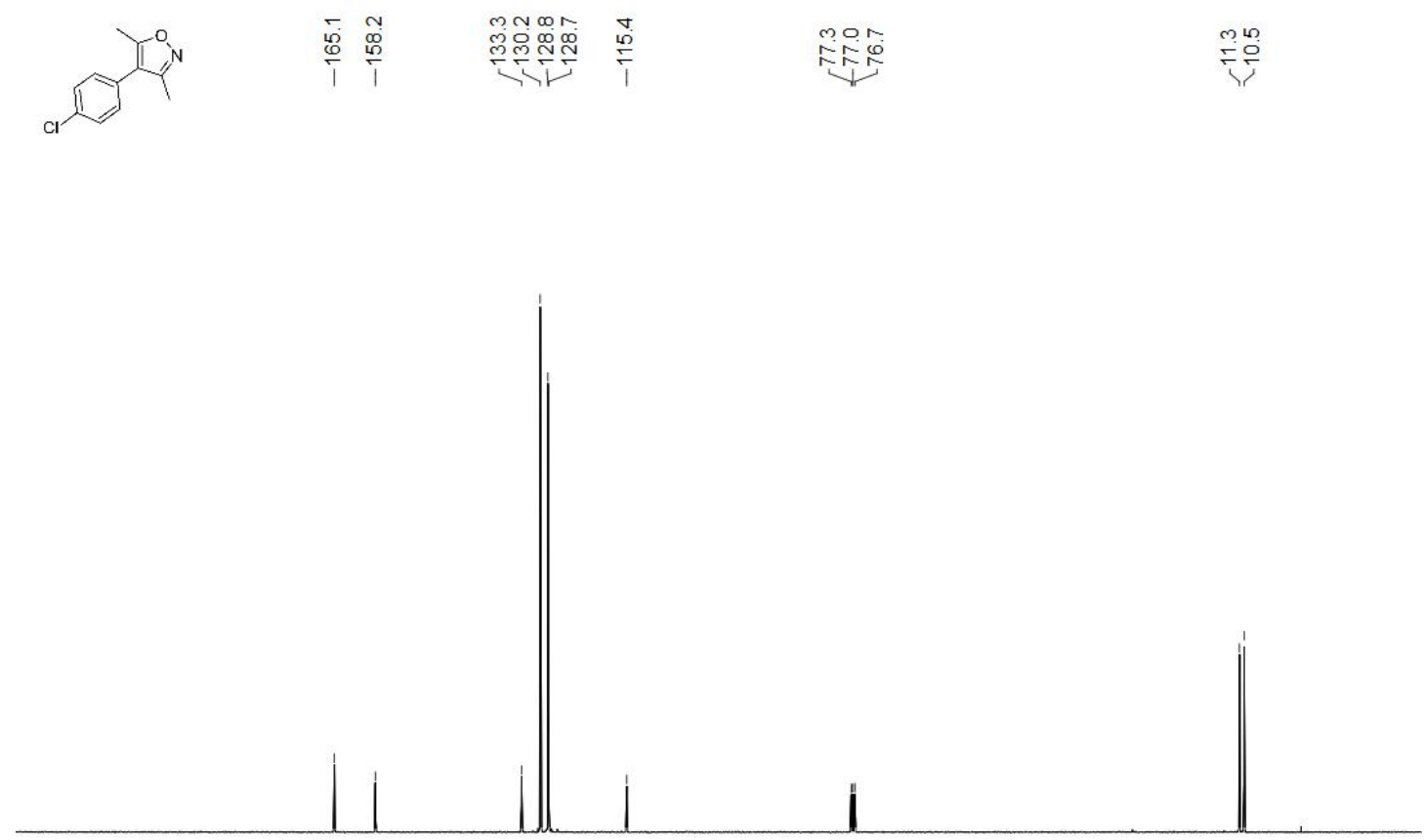

$\begin{array}{llllllllllllllllllllll}210 & 200 & 190 & 180 & 170 & 160 & 150 & 140 & 130 & 120 & 110 \begin{array}{c}100 \\ \mathrm{f} 1(\mathrm{ppm})\end{array} & 90 & 80 & 70 & 60 & 50 & 40 & 30 & 20 & 10 & 0 & -10\end{array}$ 
Figure S99. The ${ }^{1}$ H NMR spectrums of 4-(6-chloropyridin-3-yl)-3,5-dimethylisoxazole (5dp)
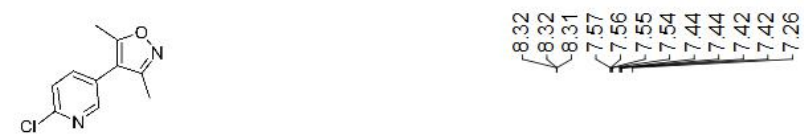

ฟัก

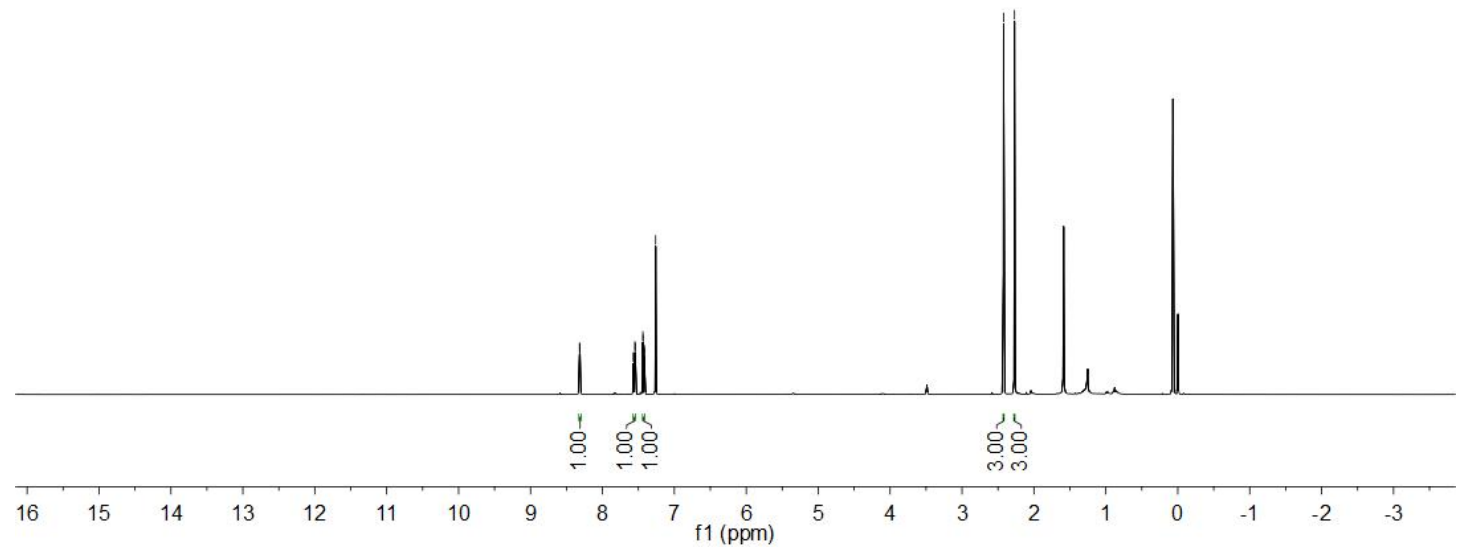

Figure S100. The ${ }^{13} \mathrm{C}$ NMR spectrums of 4-(6-chloropyridin-3-yl)-3,5-dimethylisoxazole (5dp)

近in

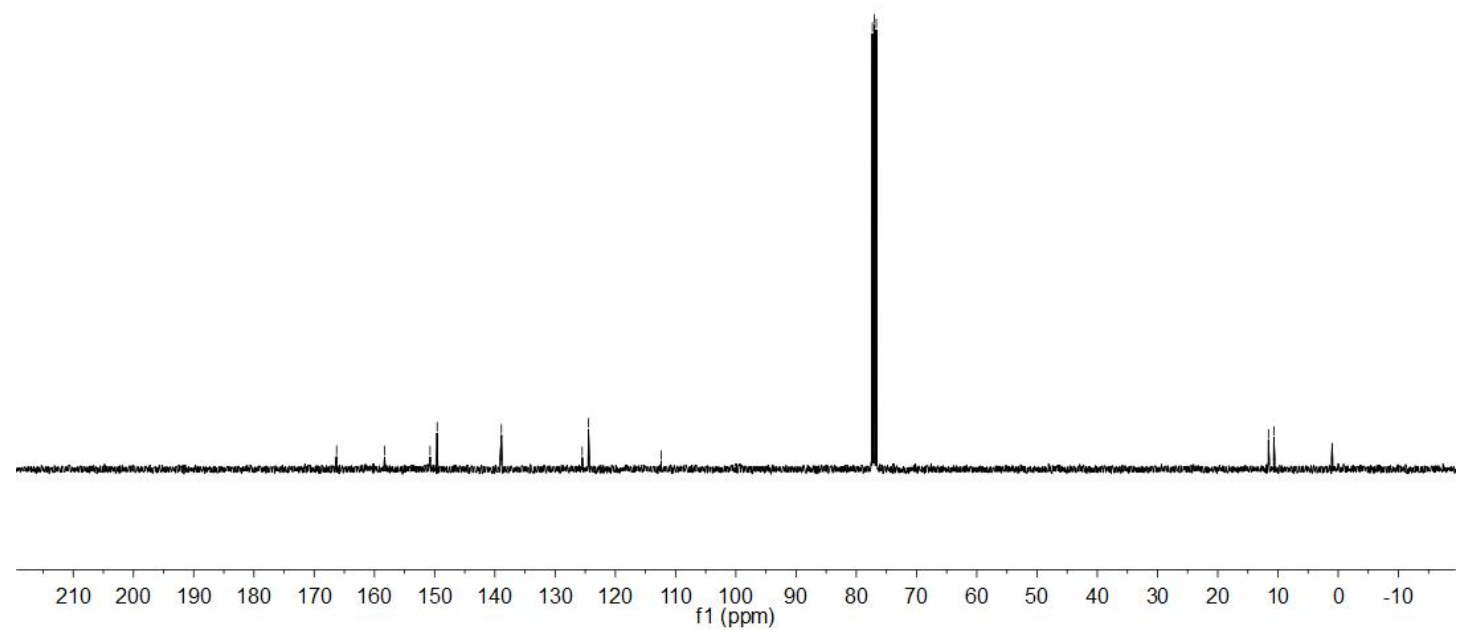


Figure S101. The ${ }^{1} \mathrm{H}$ NMR spectrums of 2-chloro-5-(1-methyl-1H-imidazol-5-yl)pyridine (5ep)

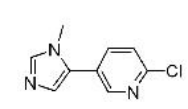

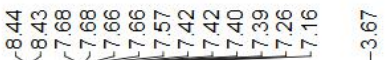

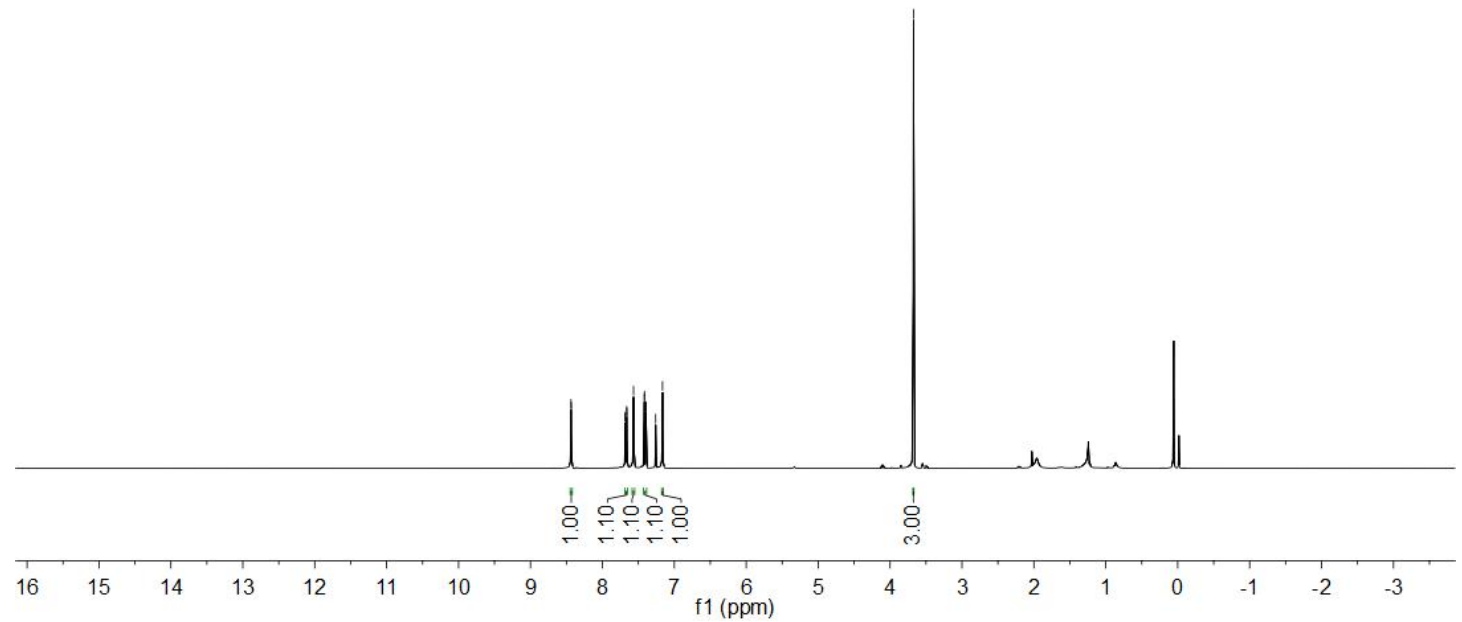

Figure S102. The ${ }^{13} \mathrm{C}$ NMR spectrums of 2-chloro-5-(1-methyl-1H-imidazol-5-yl)pyridine (5ep)
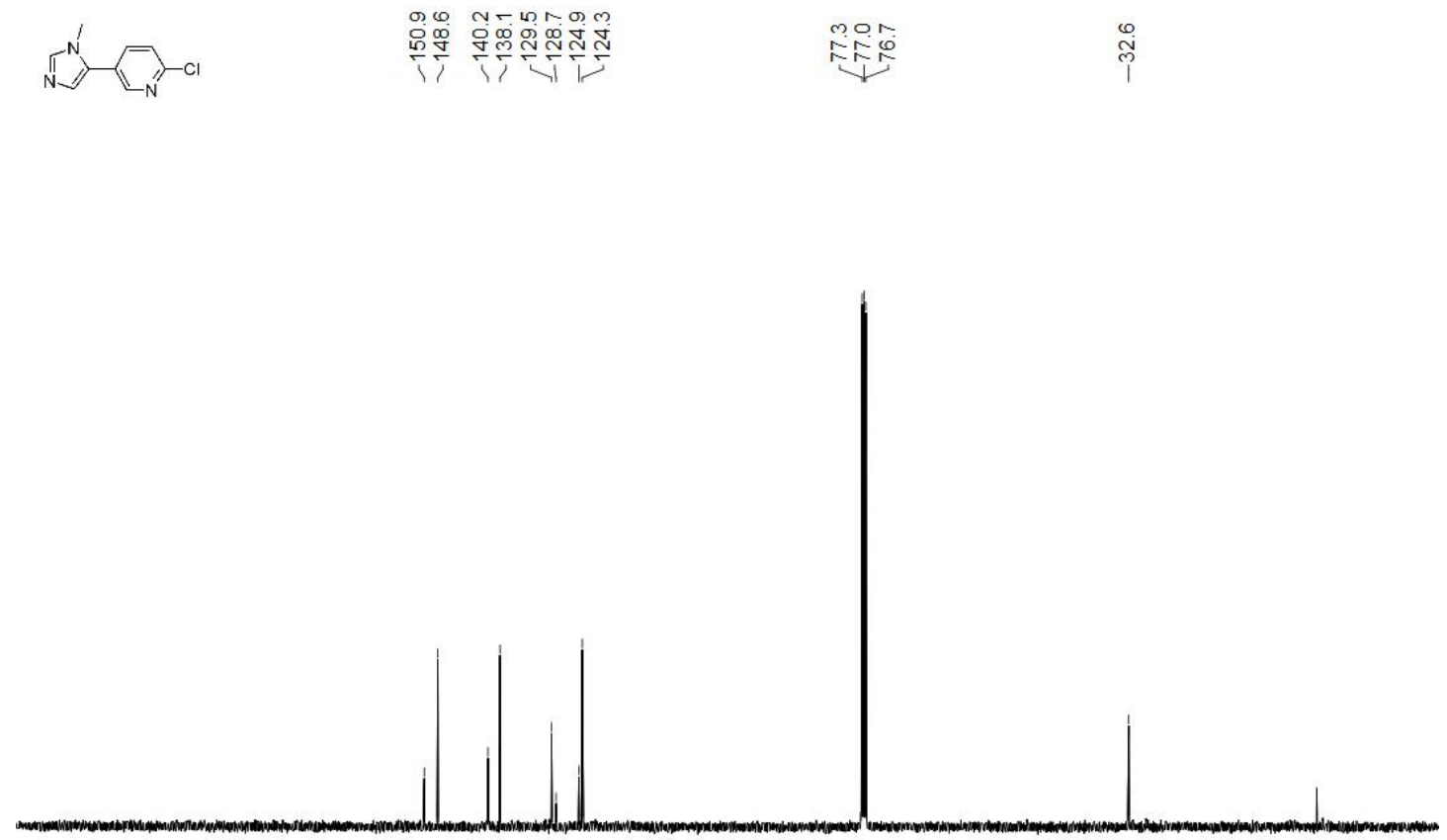

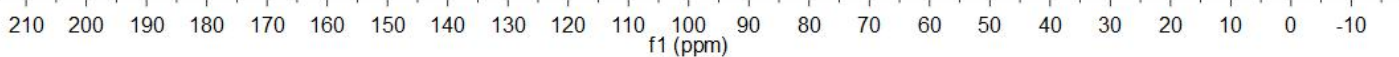


Figure S103. The ${ }^{1} \mathrm{H}$ NMR spectrums of 4-bromo-1-methyl-5-(4-nitrophenyl)-1H-pyrazole (5fa)

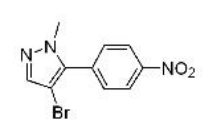

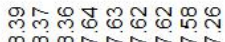

cionorintion

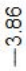

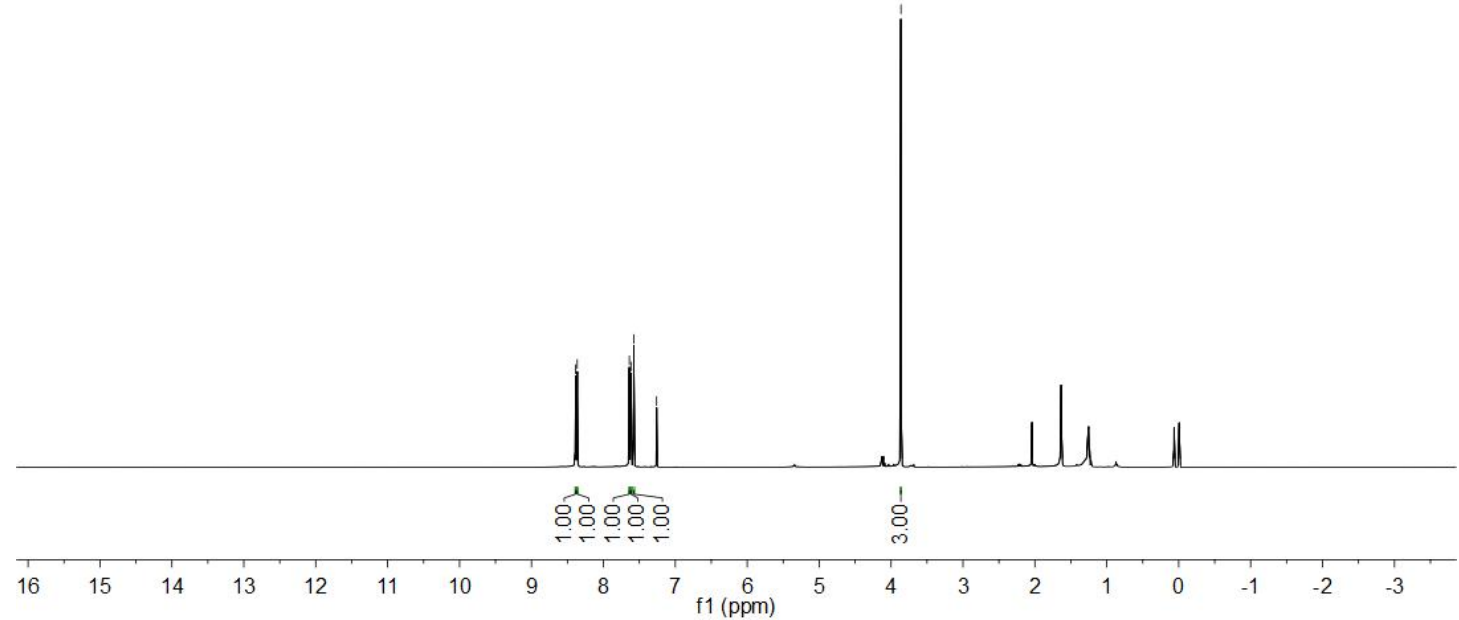

Figure S104. The ${ }^{13} \mathrm{C}$ NMR spectrums of 4-bromo-1-methyl-5-(4-nitrophenyl)-1H-pyrazole (5fa)<smiles>CCCc1cnn(C)c1-c1ccc([N+](=O)[O-])cc1</smiles>

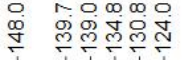

T TंT

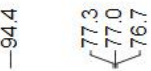

$\stackrel{\infty}{\infty}$

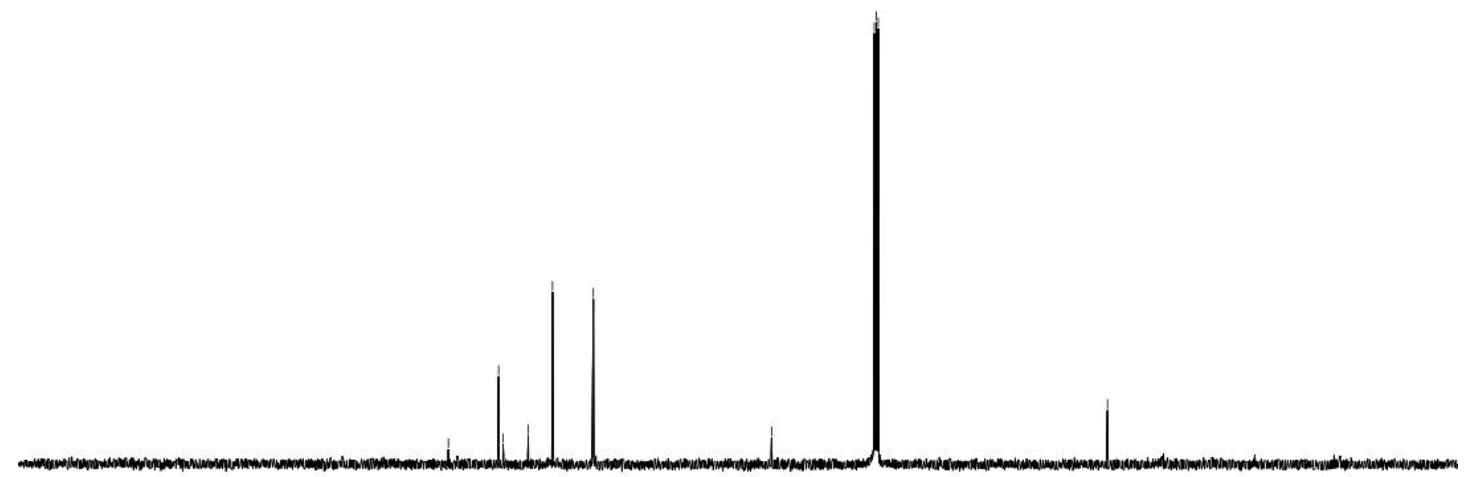

$\begin{array}{lllllllllllllllllllllll}210 & 200 & 190 & 180 & 170 & 160 & 150 & 140 & 130 & 120 & 110 \begin{array}{c}100 \\ \mathrm{f} 1(\mathrm{ppm})\end{array} & 90 & 80 & 70 & 60 & 50 & 40 & 30 & 20 & 10 & 0 & -10\end{array}$ 
Figure S105. The ${ }^{1} \mathrm{H}$ NMR spectrums of 4-(4-bromo-1-methyl-1H-pyrazol-5-yl)benzonitrile (5fb)
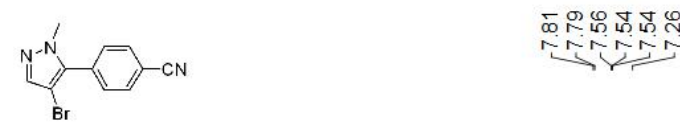

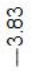

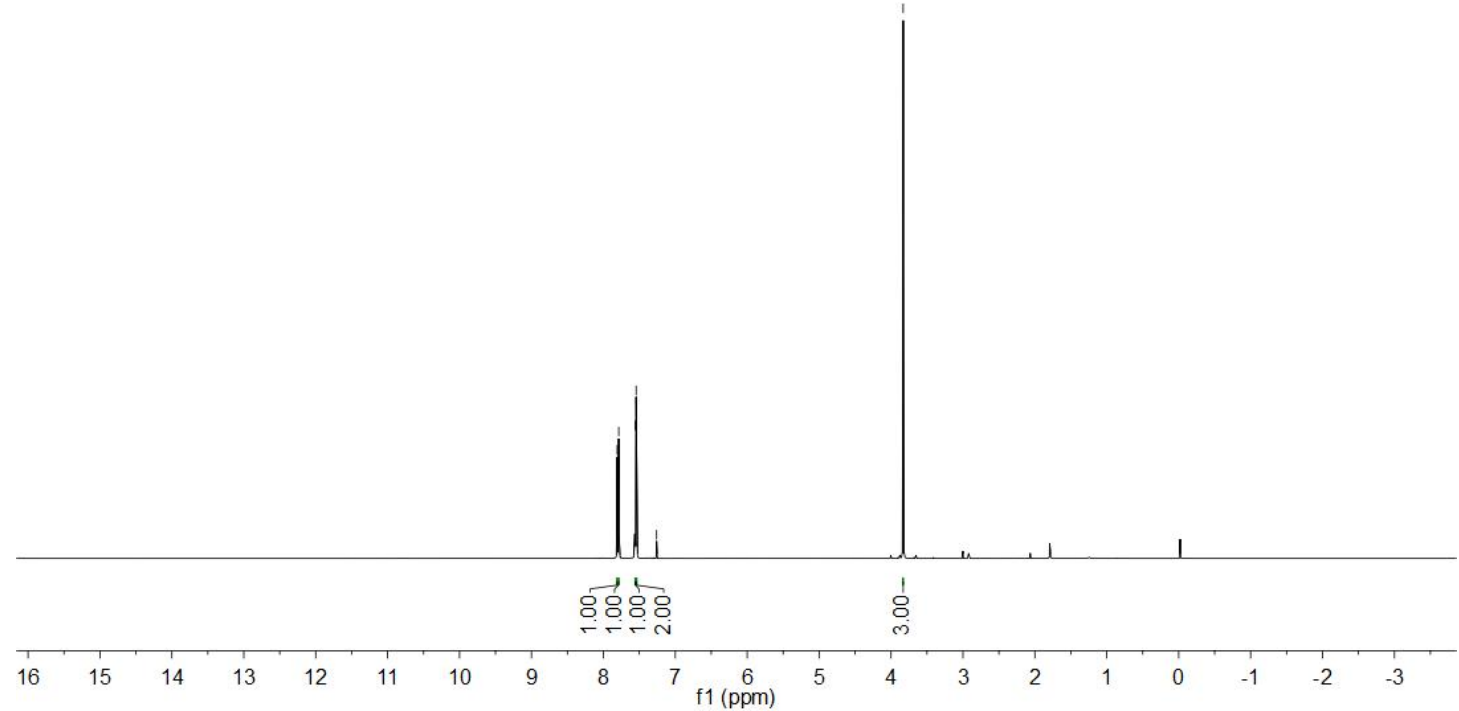

Figure S106. The ${ }^{13} \mathrm{C}$ NMR spectrums of 4-(4-bromo-1-methyl-1H-pyrazol-5-yl)benzonitrile (5fb)<smiles>CCc1cnn(C)c1-c1ccc(C#N)cc1</smiles>
कृ

家

$\stackrel{\substack{\infty \\ \oplus}}{\uparrow}$

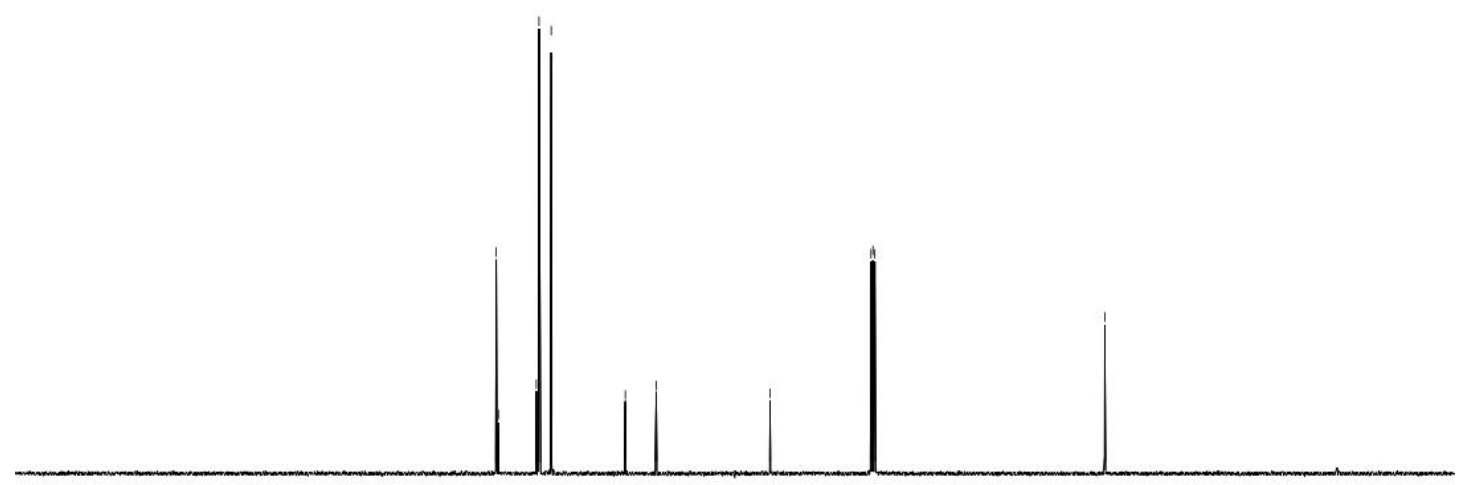

$\begin{array}{lllllllllllllllllllllll}210 & 200 & 190 & 180 & 170 & 160 & 150 & 140 & 130 & 120 & 110 \begin{array}{l}100 \\ \mathrm{f} 1(\mathrm{ppm})\end{array} & 90 & 80 & 70 & 60 & 50 & 40 & 30 & 20 & 10 & 0 & -10\end{array}$ 
Figure S107. The ${ }^{1}$ H NMR spectrums of 4-(4-bromo-1-methyl-1H-pyrazol-5-yl)benzaldehyde (5fc)
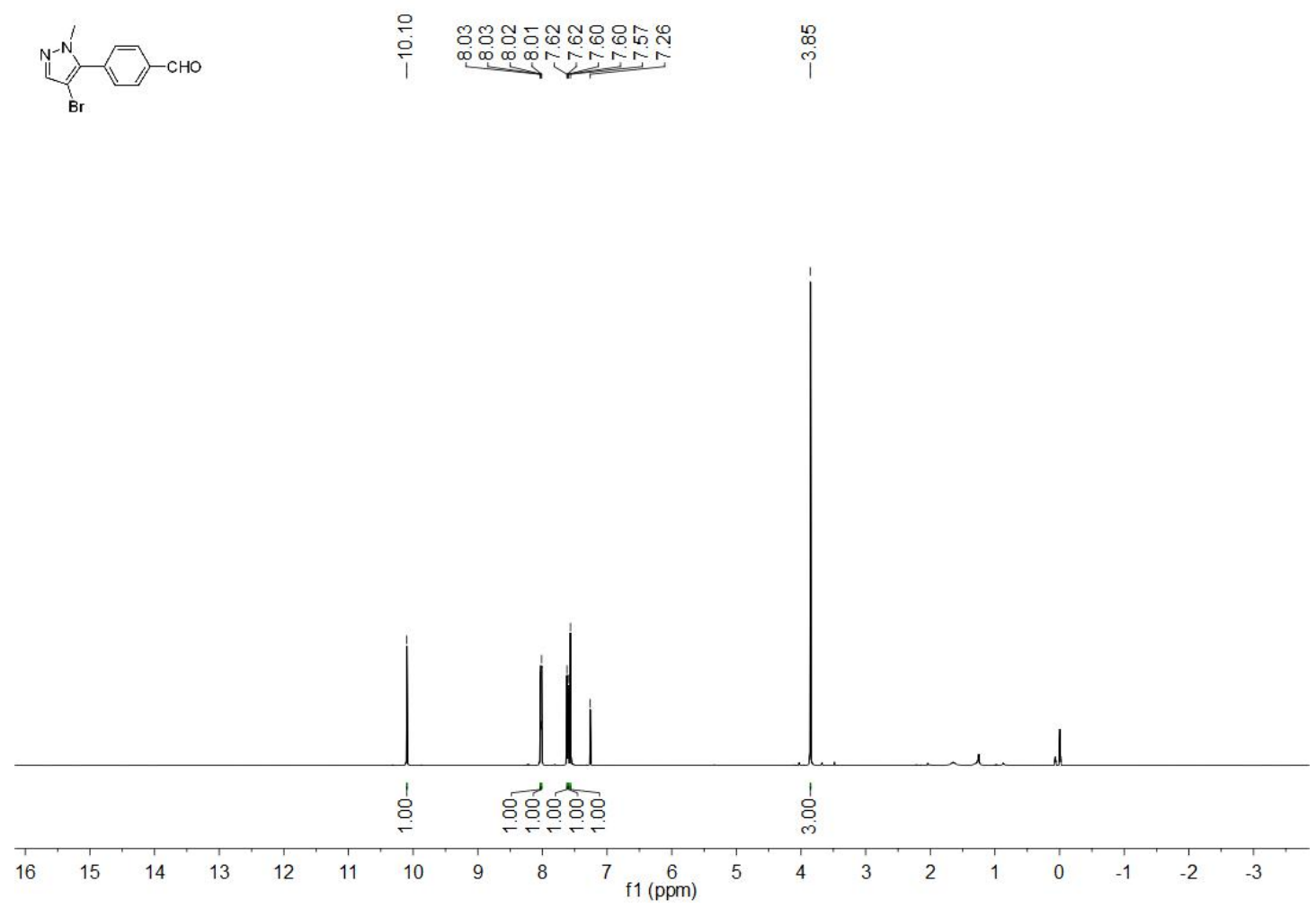

Figure S108. The ${ }^{13} \mathrm{C}$ NMR spectrums of 4-(4-bromo-1-methyl-1H-pyrazol-5-yl)benzaldehyde (5fc)
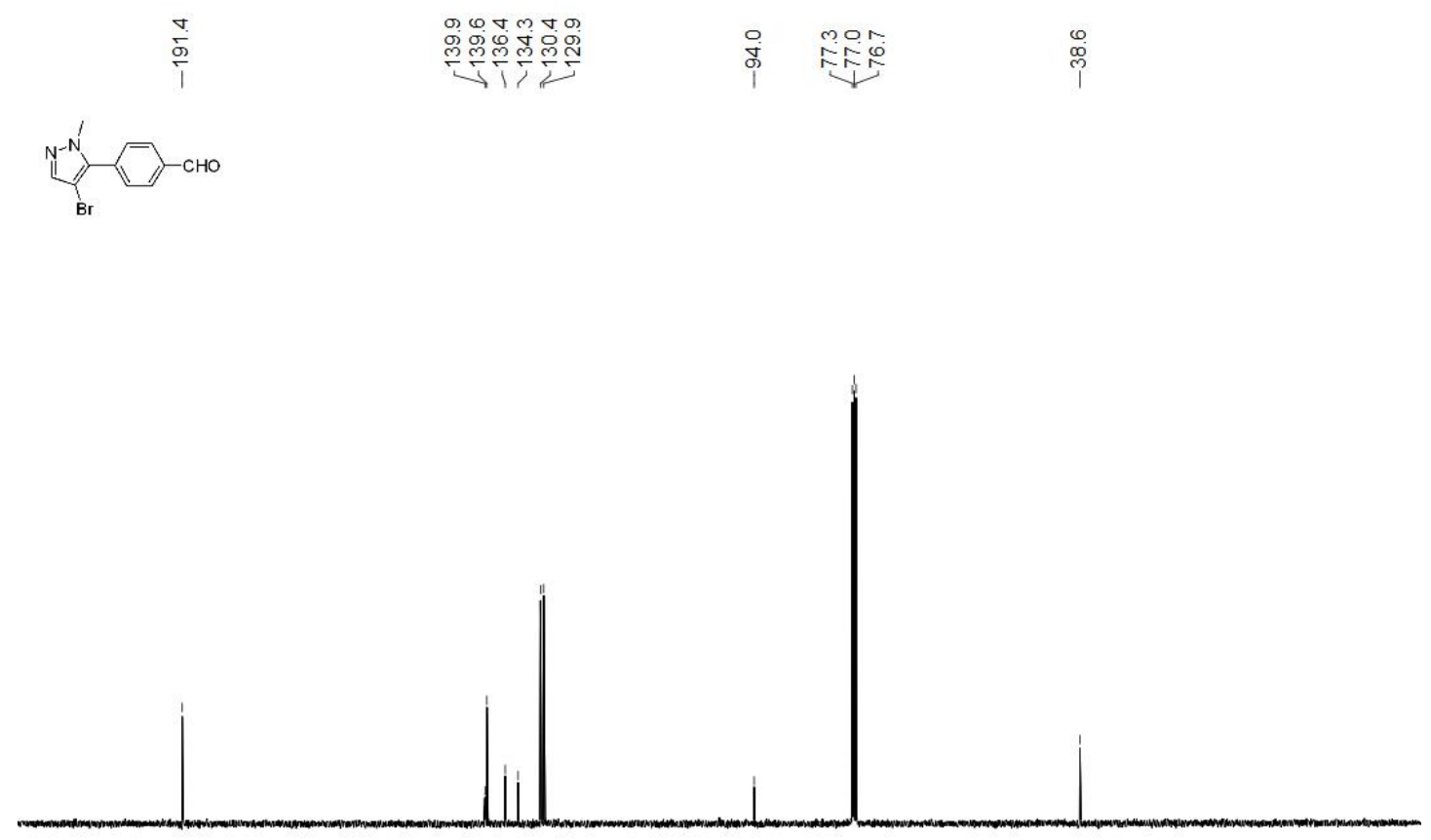

$\begin{array}{lllllllllllllllllllllll}210 & 200 & 190 & 180 & 170 & 160 & 150 & 140 & 130 & 120 & 110 \begin{array}{c}100 \\ \mathrm{f} 1(\mathrm{ppm})\end{array} & 90 & 80 & 70 & 60 & 50 & 40 & 30 & 20 & 10 & 0 & -10\end{array}$ 
Figure S109. The ${ }^{1}$ H NMR spectrums of 4-bromo-1-methyl-5-phenyl-1H-pyrazole (5ff)
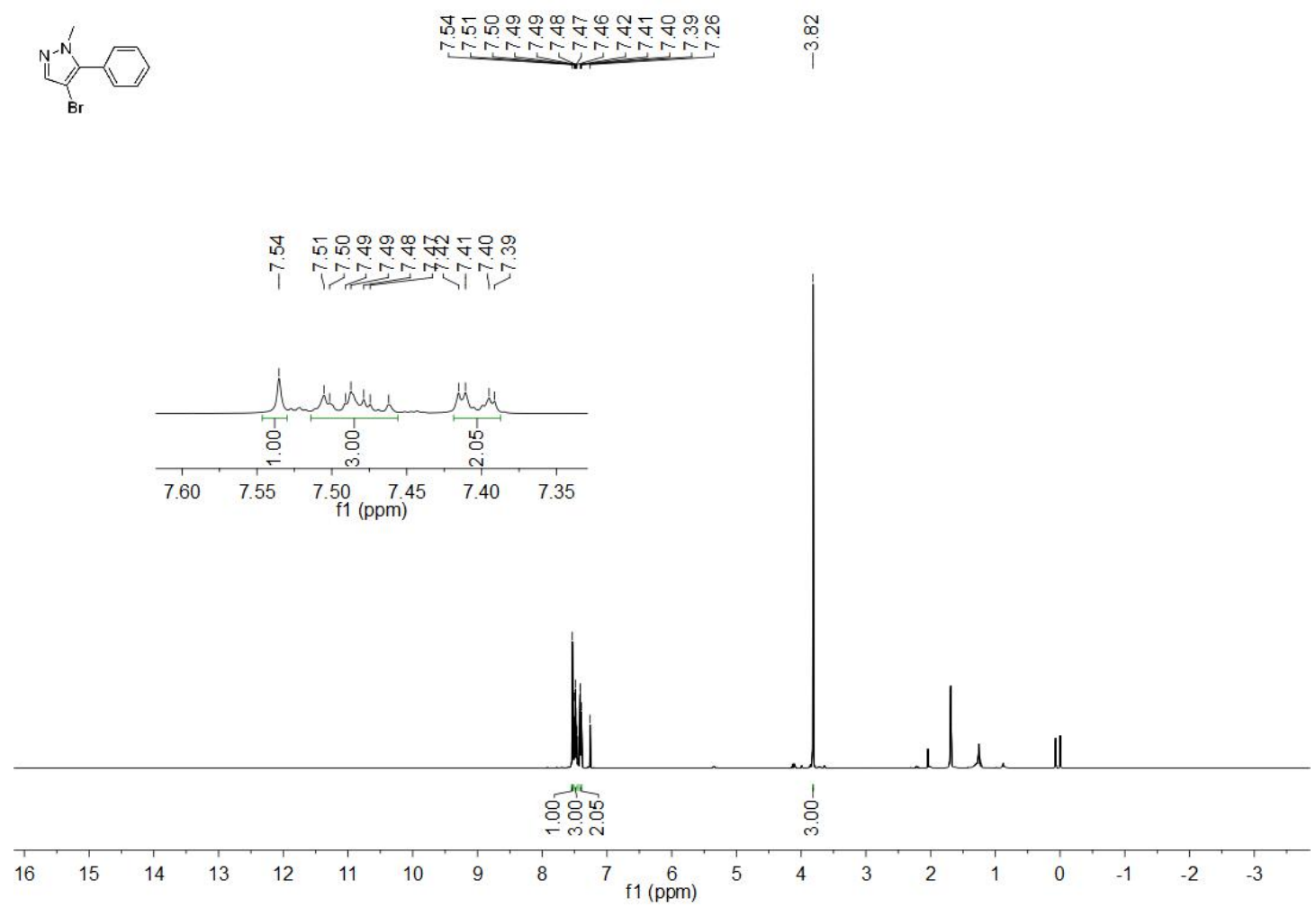

Figure S110. The ${ }^{13} \mathrm{C}$ NMR spectrums of 4-bromo-1-methyl-5-phenyl-1H-pyrazole (5ff)
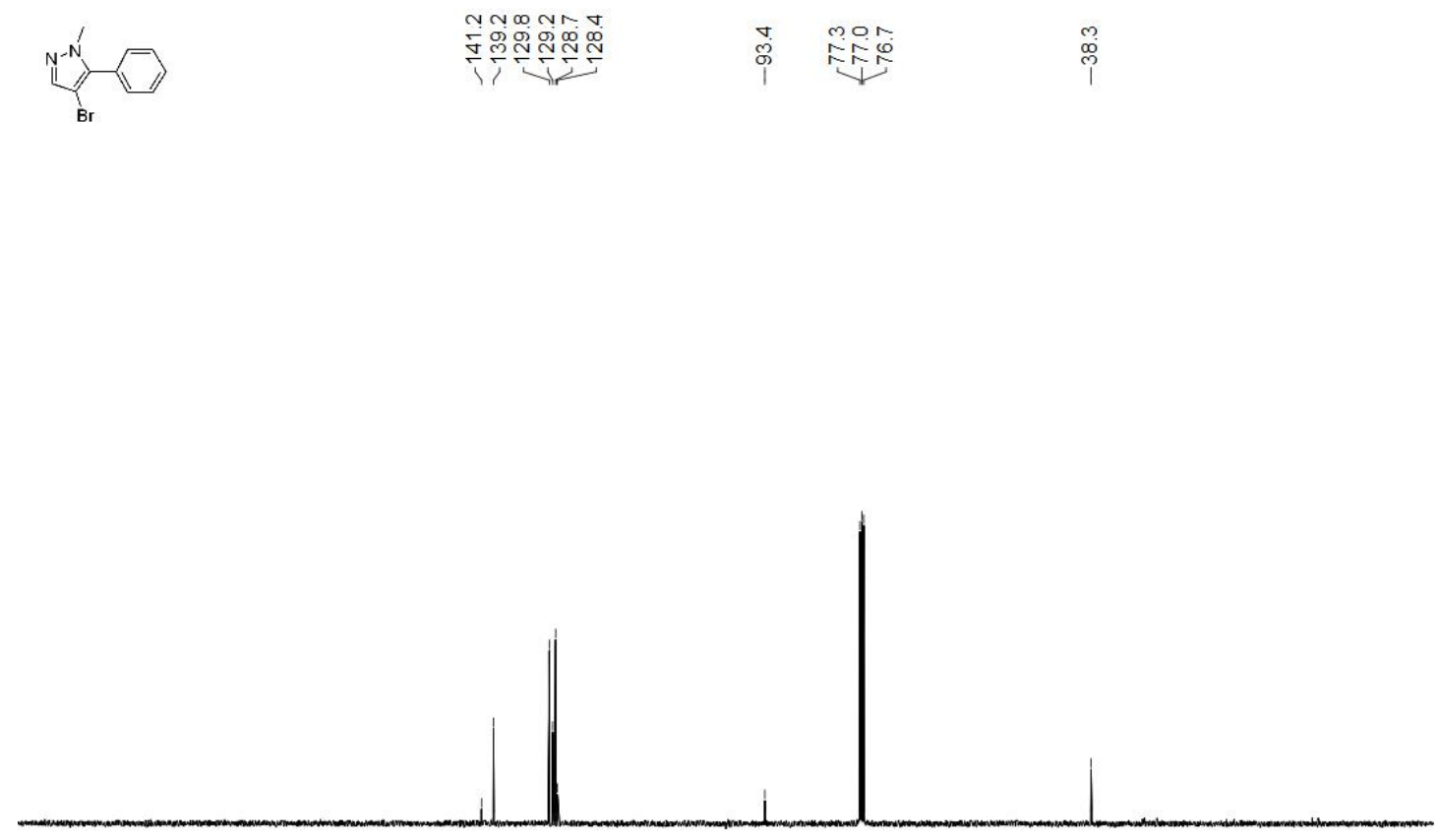

$\begin{array}{lllllllllllllllllllllll}210 & 200 & 190 & 180 & 170 & 160 & 150 & 140 & 130 & 120 & 110 & \begin{array}{c}100 \\ \mathrm{f} 1(\mathrm{ppm})\end{array} & 90 & 80 & 70 & 60 & 50 & 40 & 30 & 20 & 10 & 0 & -10\end{array}$ 
Figure S111. The ${ }^{1}$ H NMR spectrums of 5-(4-bromo-1-methyl-1H-pyrazol-5-yl)-2-methylpyridine (5fi)

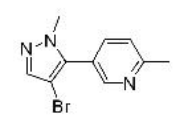

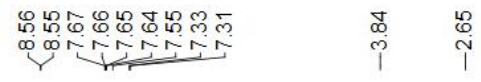

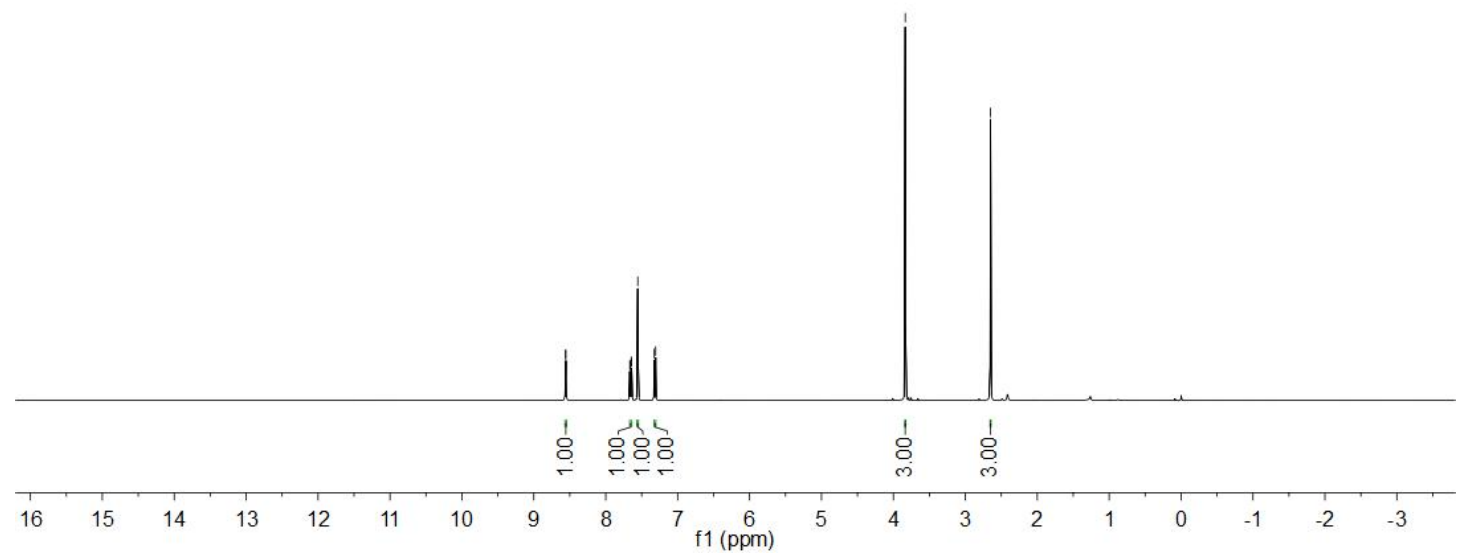

Figure S112. The ${ }^{13} \mathrm{C}$ NMR spectrums of 5-(4-bromo-1-methyl-1H-pyrazol-5-yl)-2-methylpyridine (5fi)
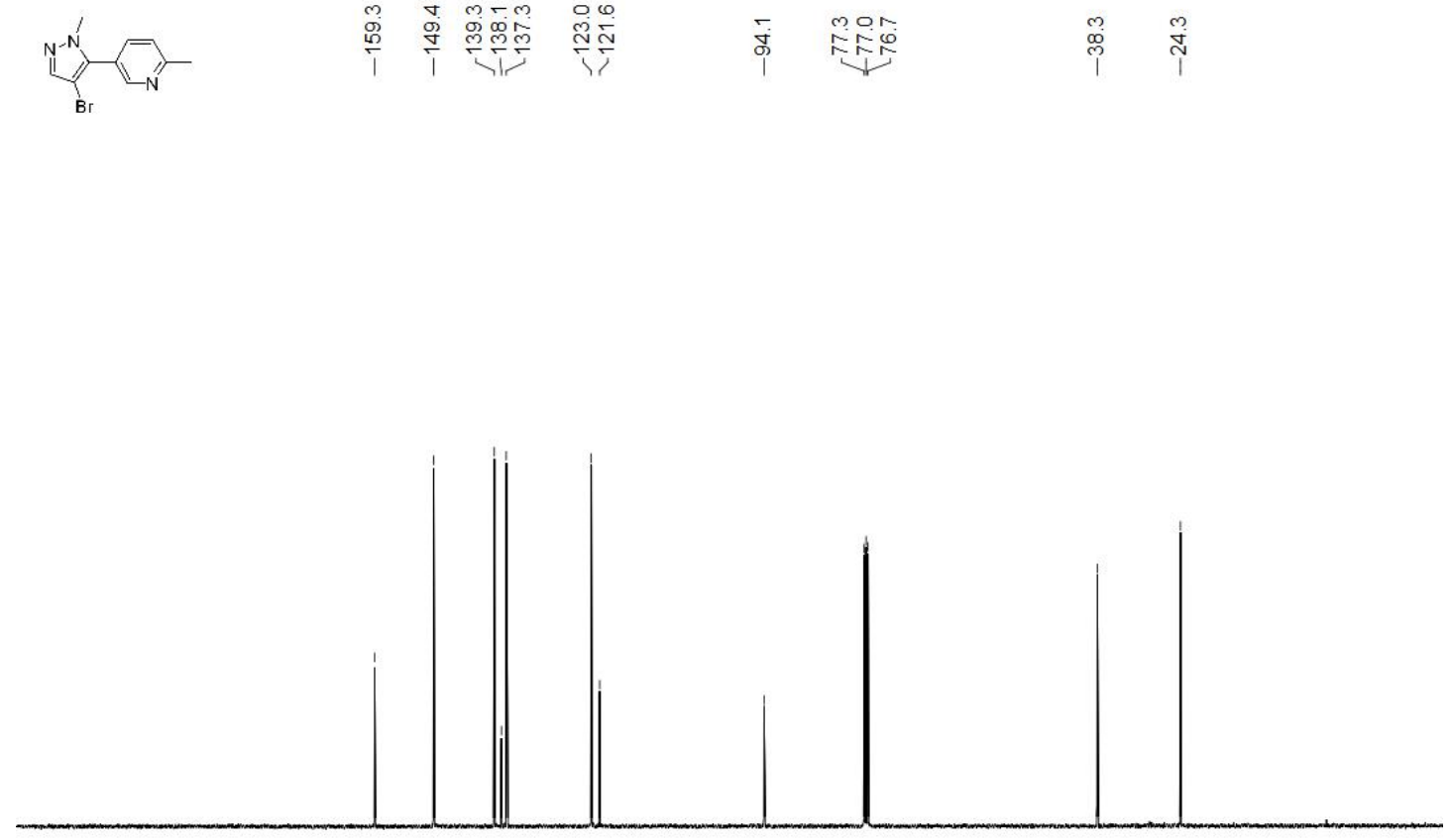

$\begin{array}{lllllllllllllllllllllll}210 & 200 & 190 & 180 & 170 & 160 & 150 & 140 & 130 & 120 & 110 & \begin{array}{c}100 \\ \mathrm{f} 1(\mathrm{ppm})\end{array} & 90 & 80 & 70 & 60 & 50 & 40 & 30 & 20 & 10 & 0 & -10\end{array}$ 
Figure S113. The ${ }^{1} \mathrm{H}$ NMR spectrums of 4-bromo-1-methyl-5-(naphthalen-2-yl)-1H-pyrazole (5fr)
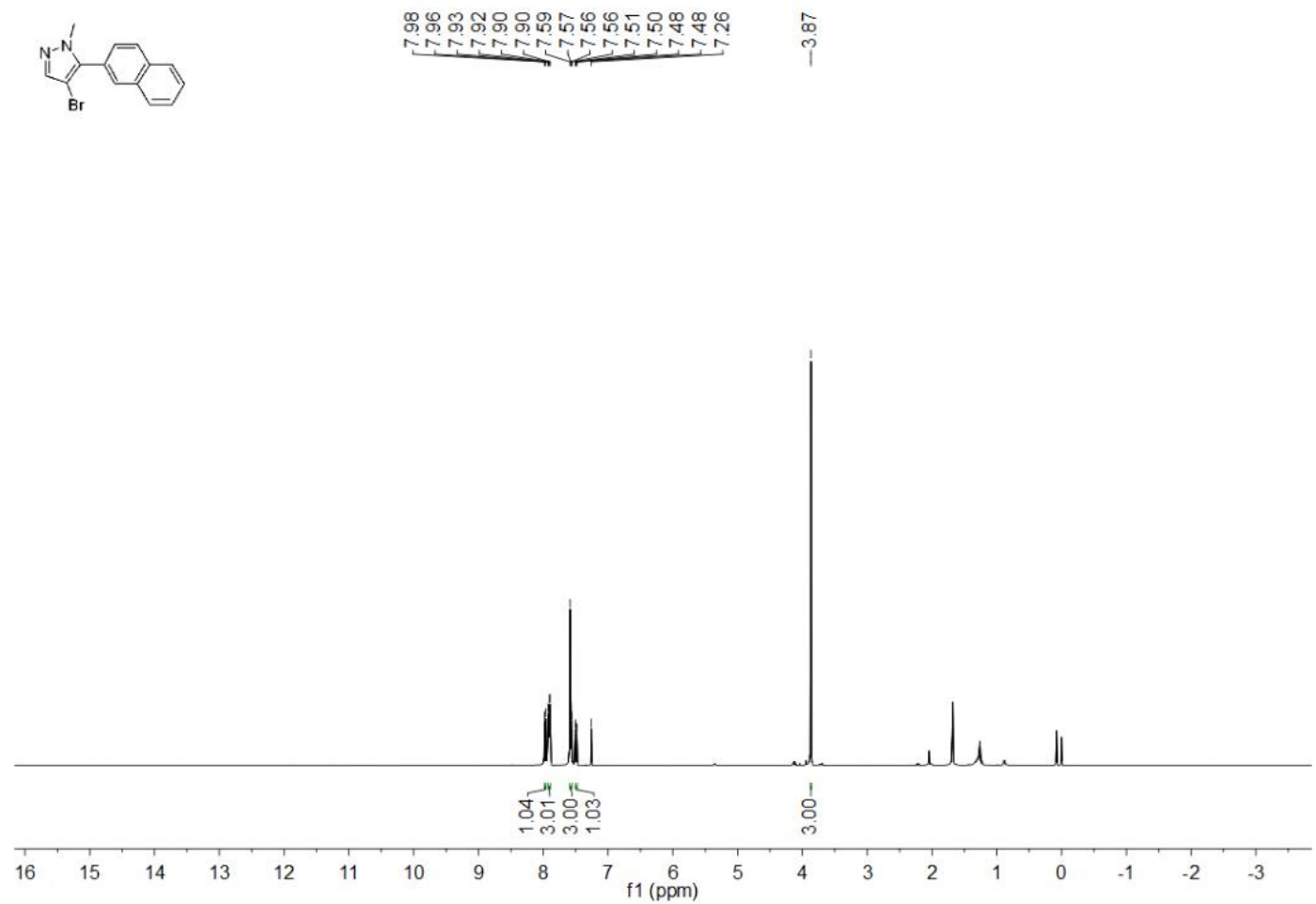

Figure S114. The ${ }^{13} \mathrm{C}$ NMR spectrums of 4-bromo-1-methyl-5-(naphthalen-2-yl)-1H-pyrazole (5fr)

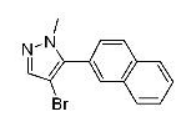

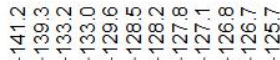

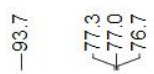

$\underset{\substack{1 \\ \infty}}{\infty}$

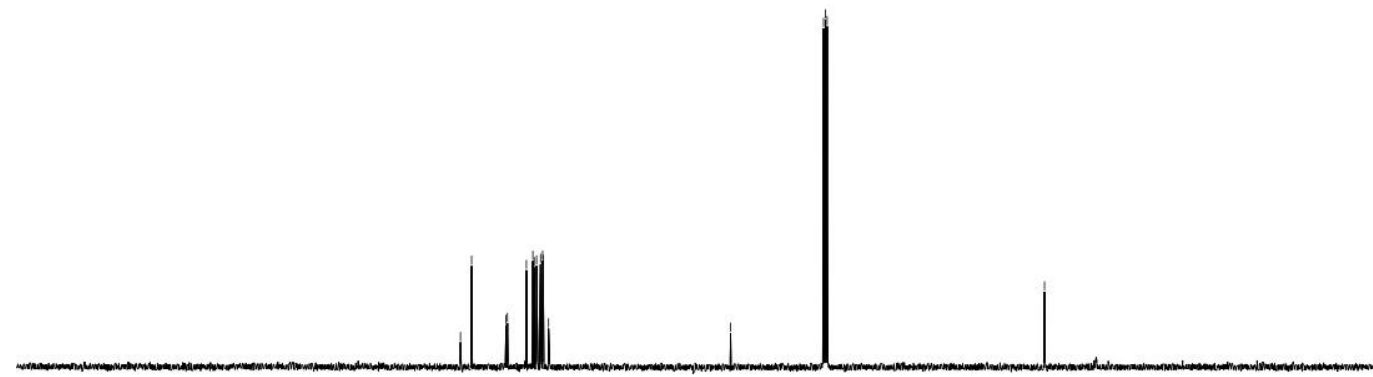

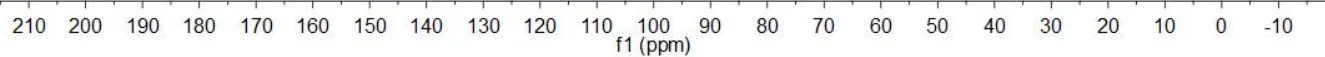


Figure S115. The ${ }^{1}$ H NMR spectrums of 5-(4-formylphenyl)-1-methyl-1H-pyrrole-2-carbaldehyde (5gc)

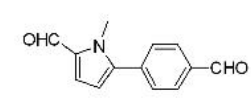

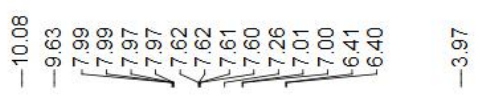

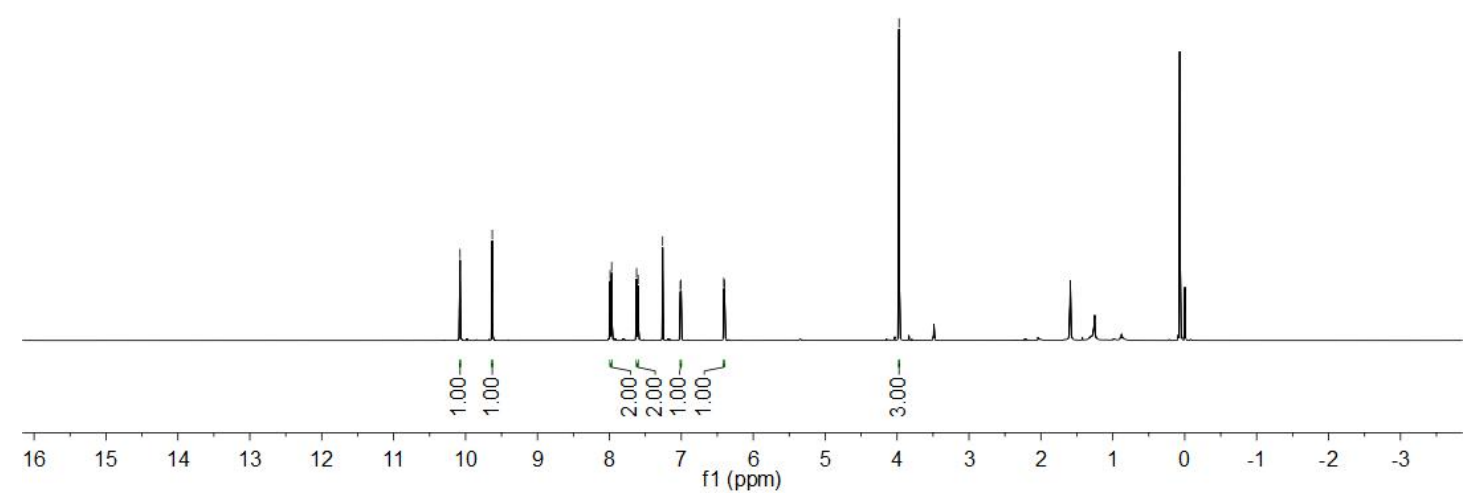

Figure S116. The ${ }^{13} \mathrm{C}$ NMR spectrums of

5-(4-formylphenyl)-1-methyl-1H-pyrrole-2-carbaldehyde (5gc)

章 家
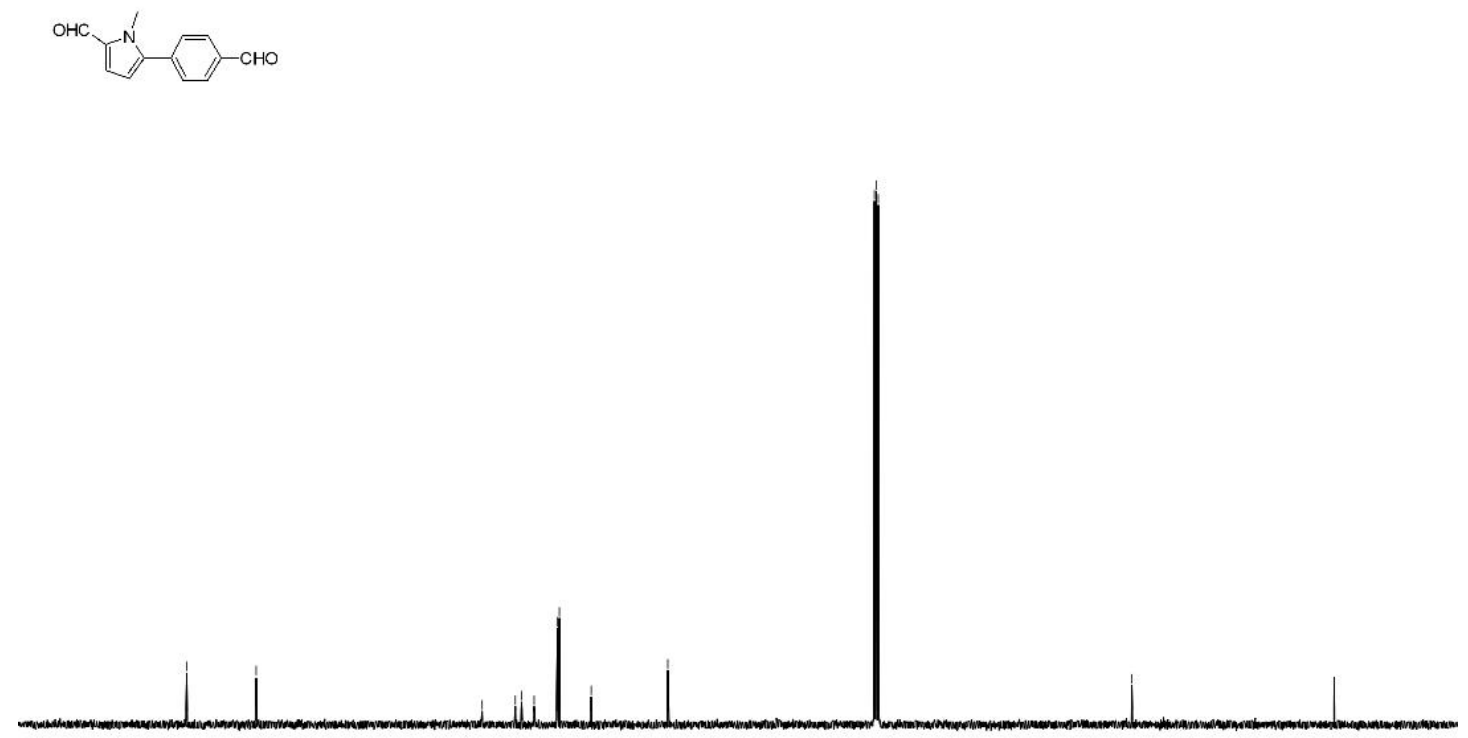

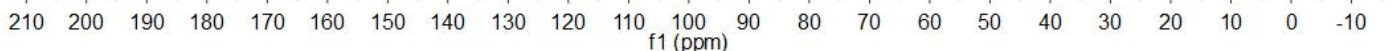


Figure S117. The ${ }^{1}$ H NMR spectrums of 1-methyl-5-(pyridin-3-yl)-1H-pyrrole-2-carbaldehyde (5gj)
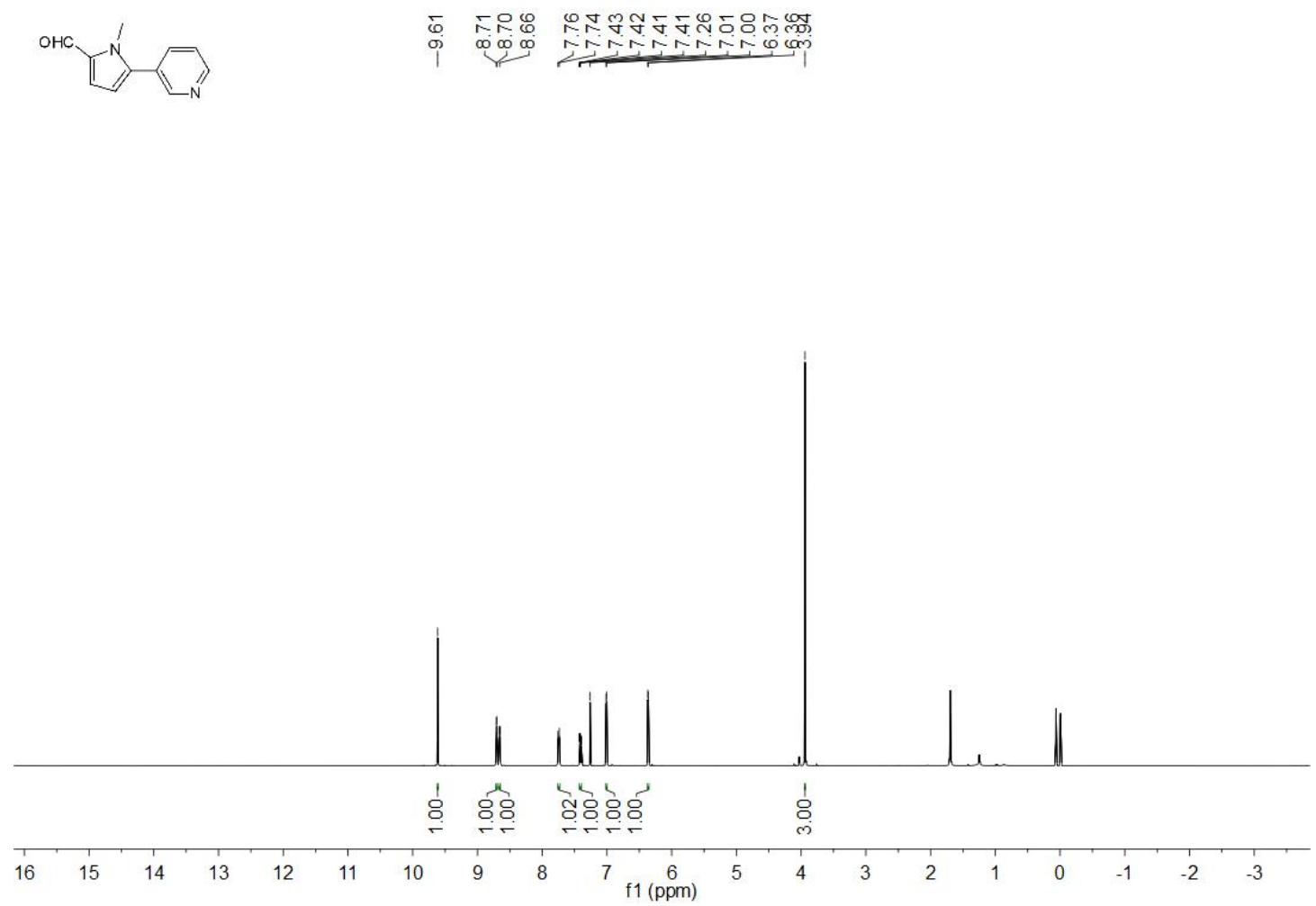

Figure S118. The ${ }^{13} \mathrm{C}$ NMR spectrums of 1-methyl-5-(pyridin-3-yl)-1H-pyrrole-2-carbaldehyde (5gj)

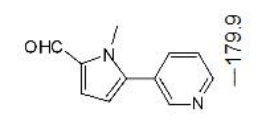
咅
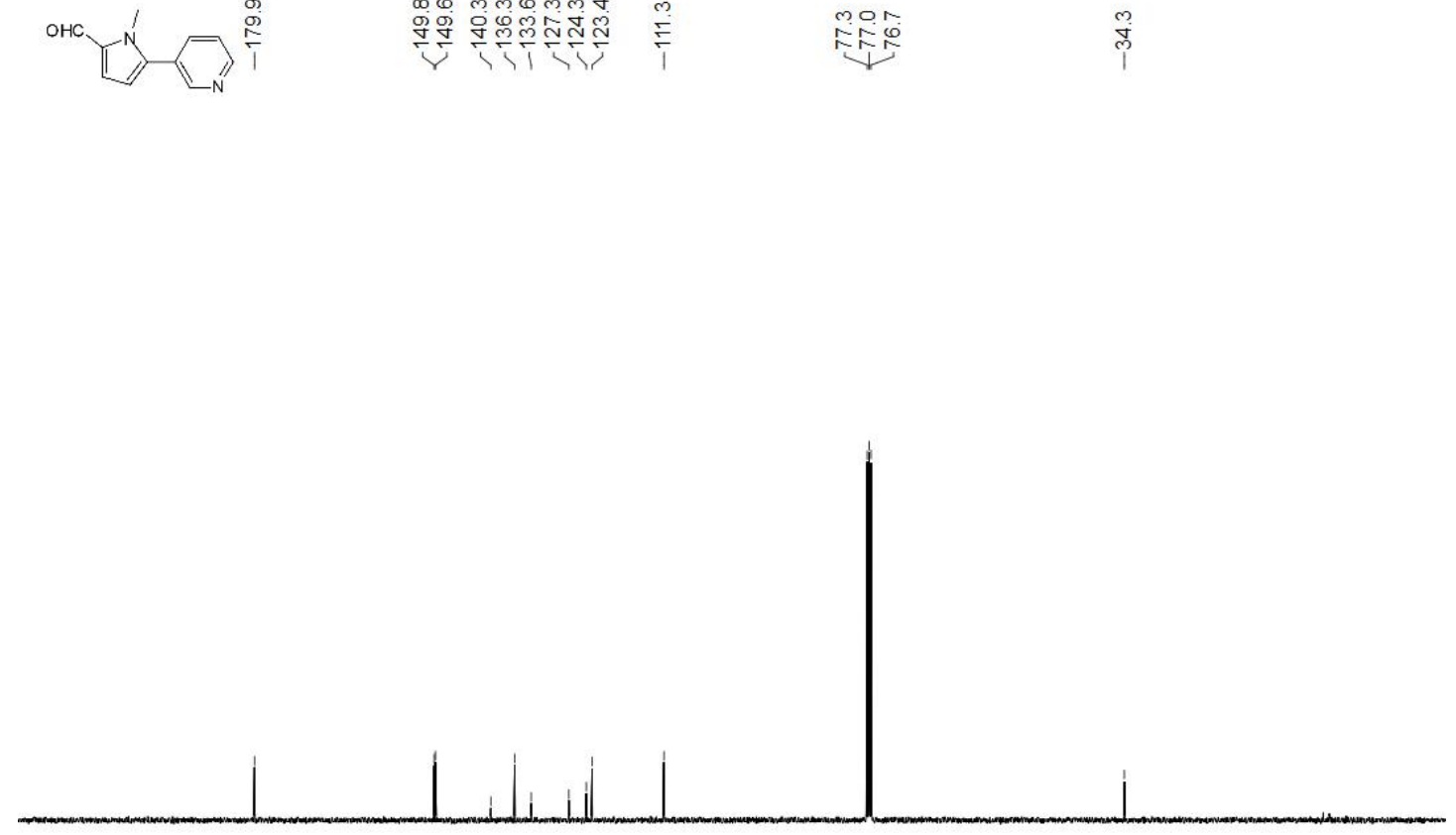

$\begin{array}{lllllllllllllllllllllll}210 & 200 & 190 & 180 & 170 & 160 & 150 & 140 & 130 & 120 & 110 \begin{array}{c}100 \\ \mathrm{f} 1(\mathrm{ppm})\end{array} & 90 & 80 & 70 & 60 & 50 & 40 & 30 & 20 & 10 & 0 & -10\end{array}$ 
Figure S119. The ${ }^{1} \mathrm{H}$ NMR spectrums of (pyrimidin-5-yl)-1H-pyrrole-2-carbaldehyde (5gq)
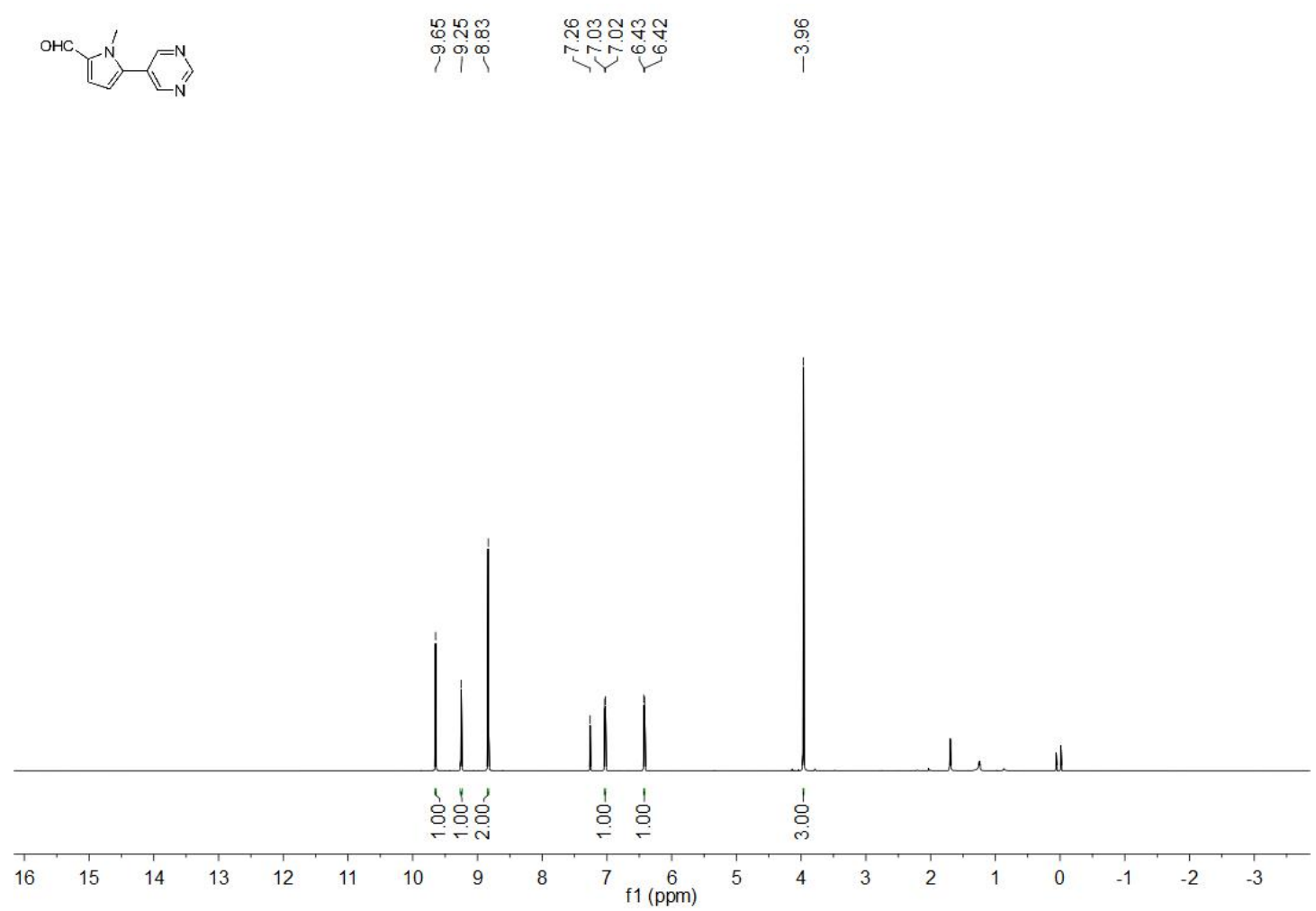

Figure S120. The ${ }^{13} \mathrm{C}$ NMR spectrums of (pyrimidin-5-yl)-1H-pyrrole-2-carbaldehyde (5gq)

OHC

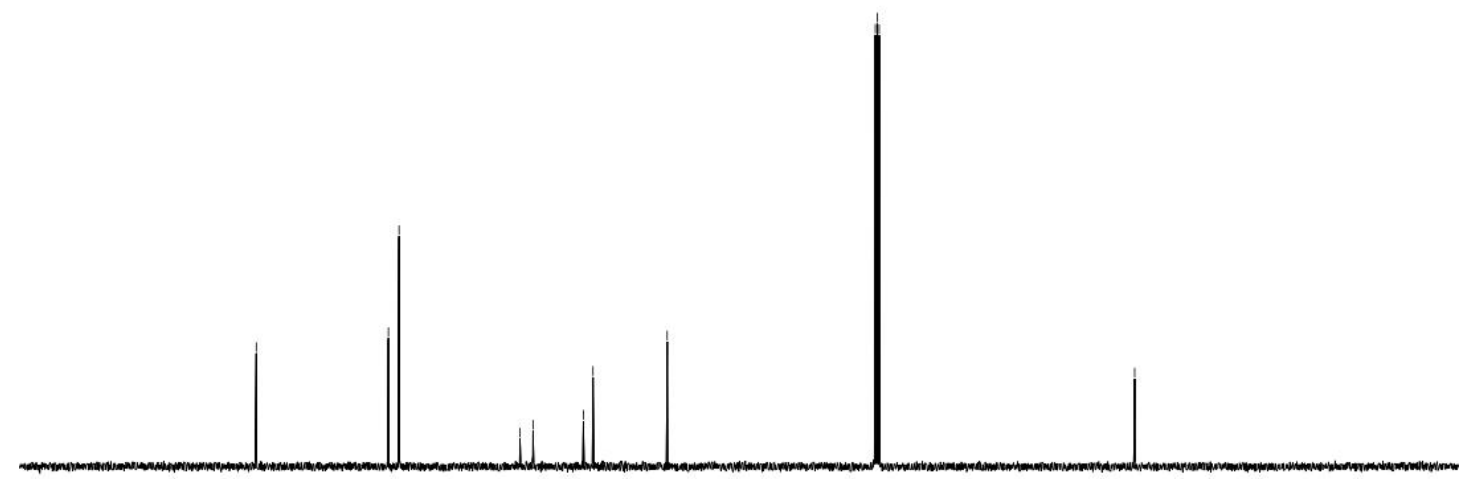

$\begin{array}{lllllllllllllllllllllll}210 & 200 & 190 & 180 & 170 & 160 & 150 & 140 & 130 & 120 & 110 \begin{array}{c}100 \\ \mathrm{f} 1(\mathrm{ppm})\end{array} & 90 & 80 & 70 & 60 & 50 & 40 & 30 & 20 & 10 & 0 & -10\end{array}$ 
Figure S121. The ${ }^{1} \mathrm{H}$ NMR spectrums of 1-methyl-2-(6-methylpyridin-3-yl)-1H-indole (5hi)
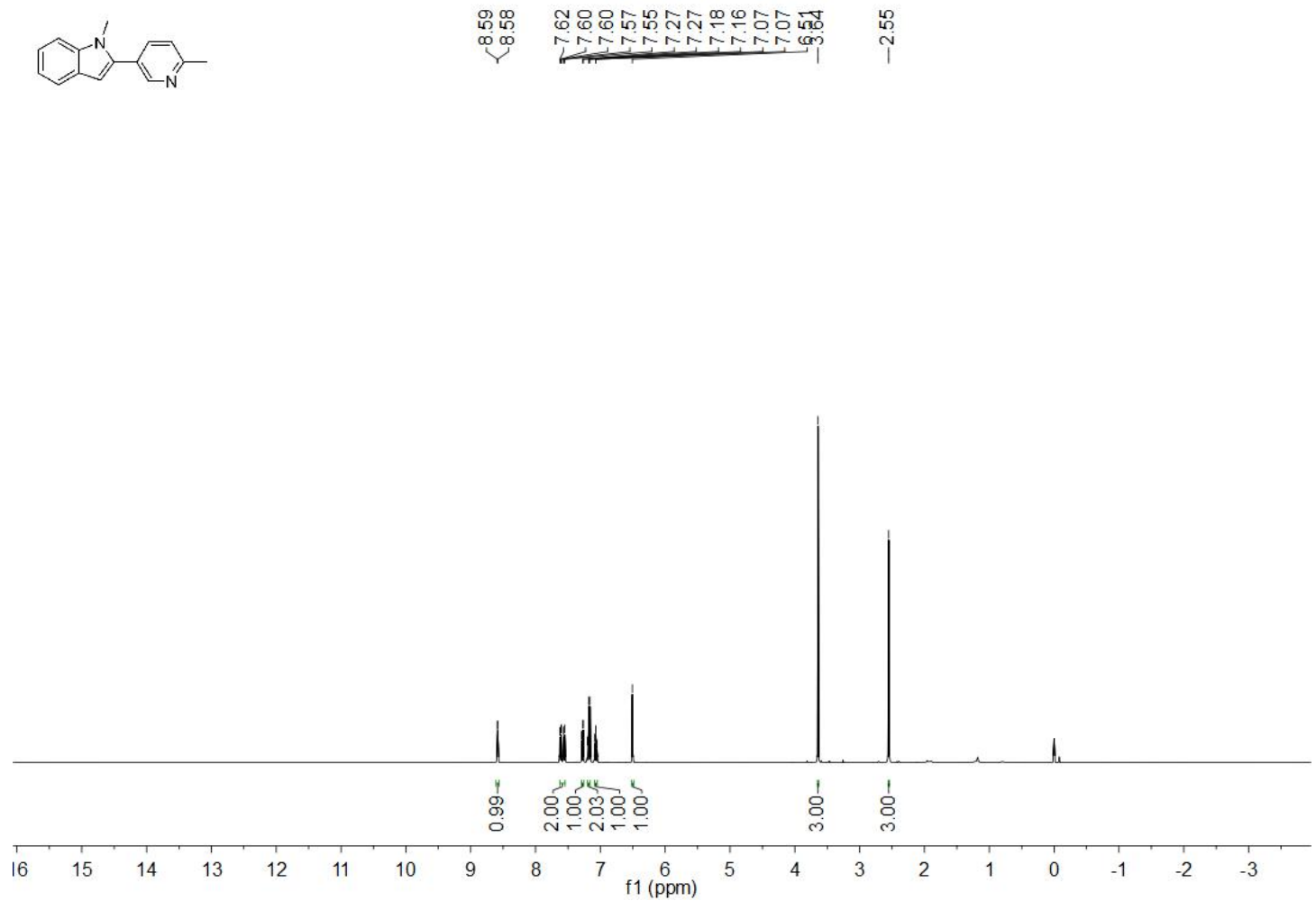

Figure S122. The ${ }^{13} \mathrm{C}$ NMR spectrums of 1-methyl-2-(6-methylpyridin-3-yl)-1H-indole (5hi)
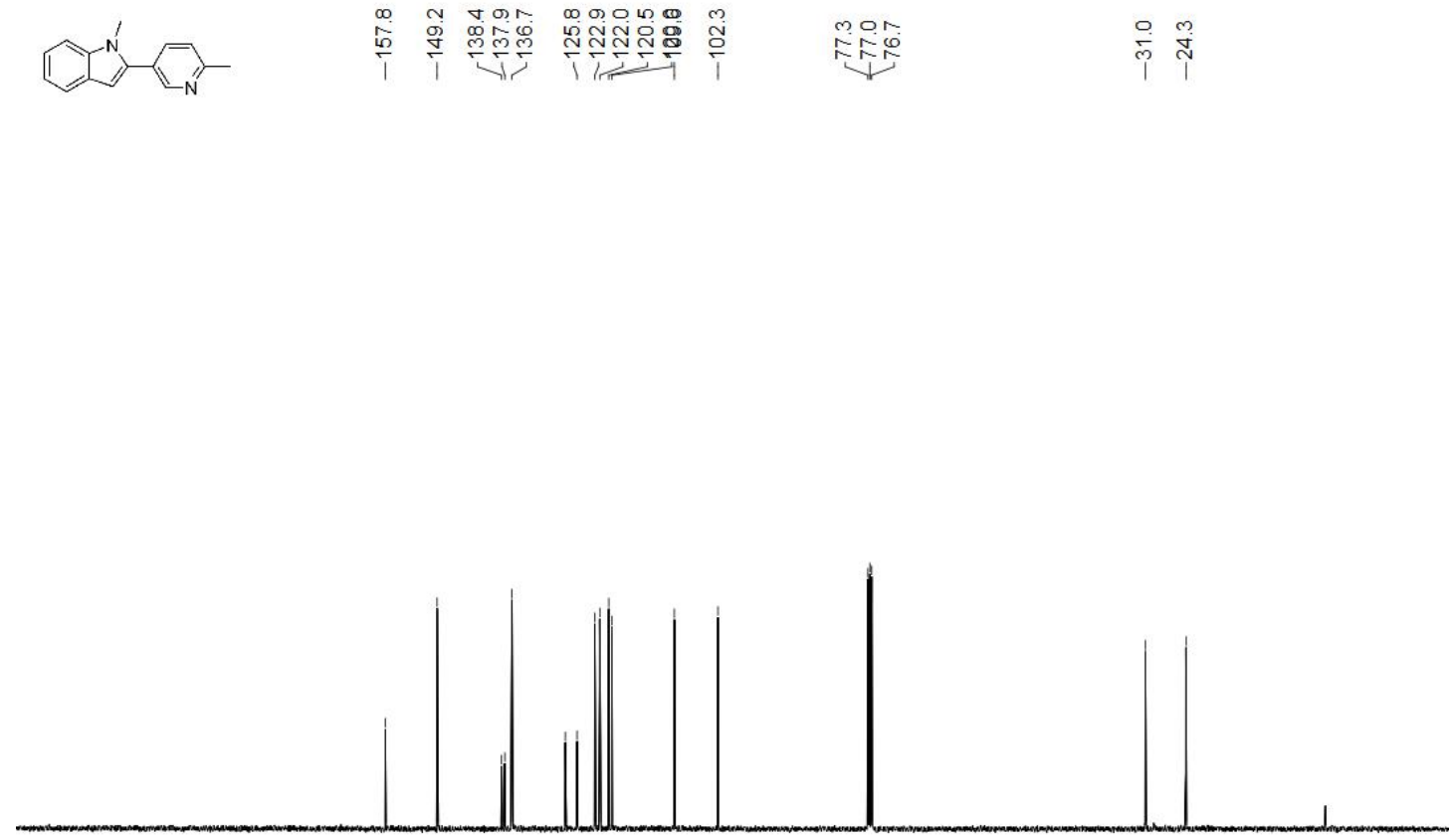

$\begin{array}{llllllllllllllllllllll}210 & 200 & 190 & 180 & 170 & 160 & 150 & 140 & 130 & 120 & 110 \begin{array}{c}100 \\ \mathrm{f} 1(\mathrm{ppm})\end{array} & 90 & 80 & 70 & 60 & 50 & 40 & 30 & 20 & 10 & 0 & -10\end{array}$ 
Figure S123. The ${ }^{1} \mathrm{H}$ NMR spectrums of

2-(4-fluorophenyl)-3-(6-methylpyridin-3-yl)imidazo[1,2-a]pyridine (5ii)
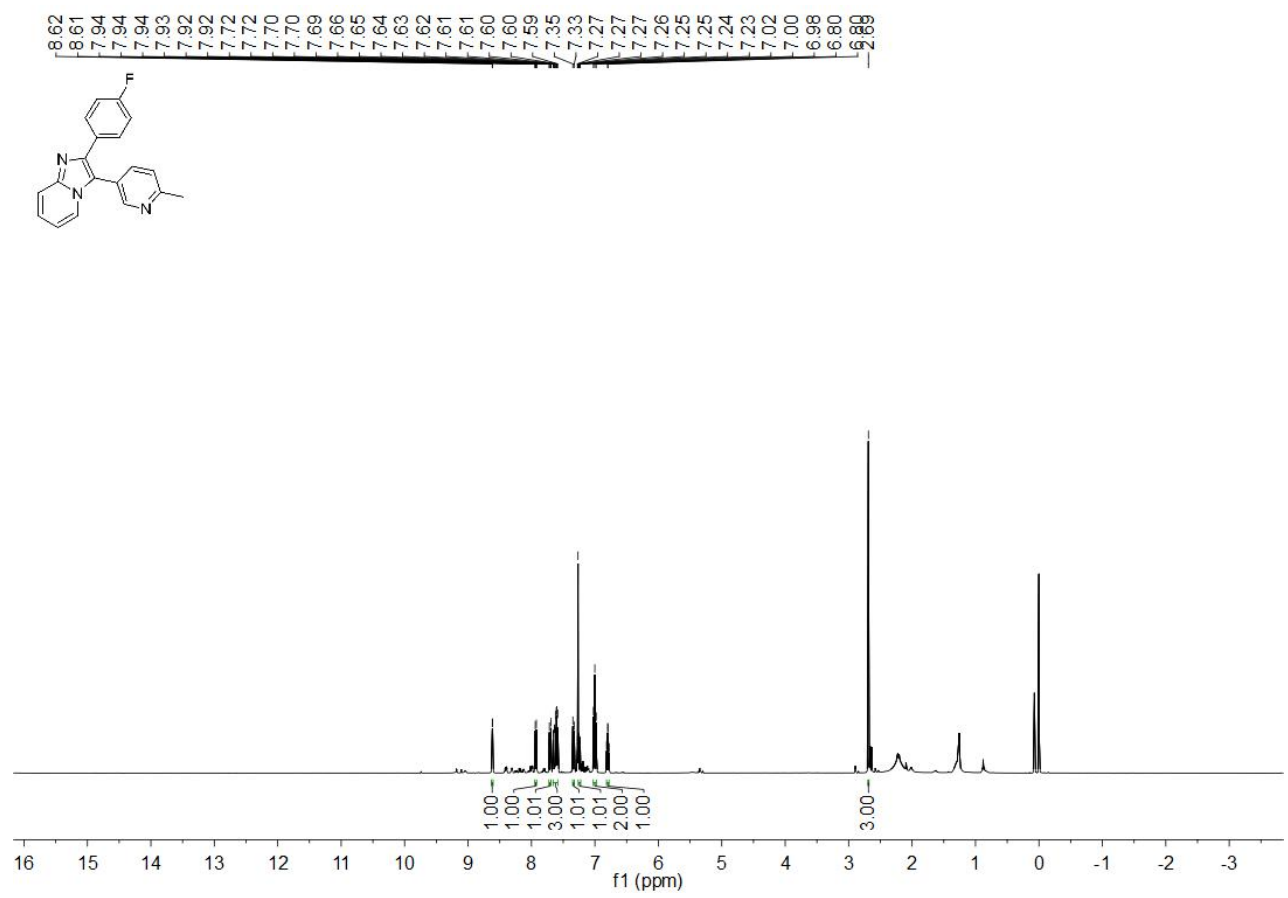

Figure S124. The ${ }^{13} \mathrm{C}$ NMR spectrums of

2-(4-fluorophenyl)-3-(6-methylpyridin-3-yl)imidazo[1,2-a]pyridine (5ii)

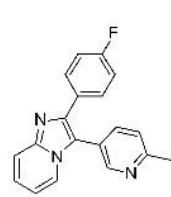

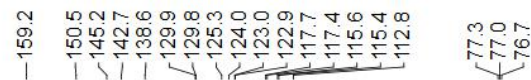

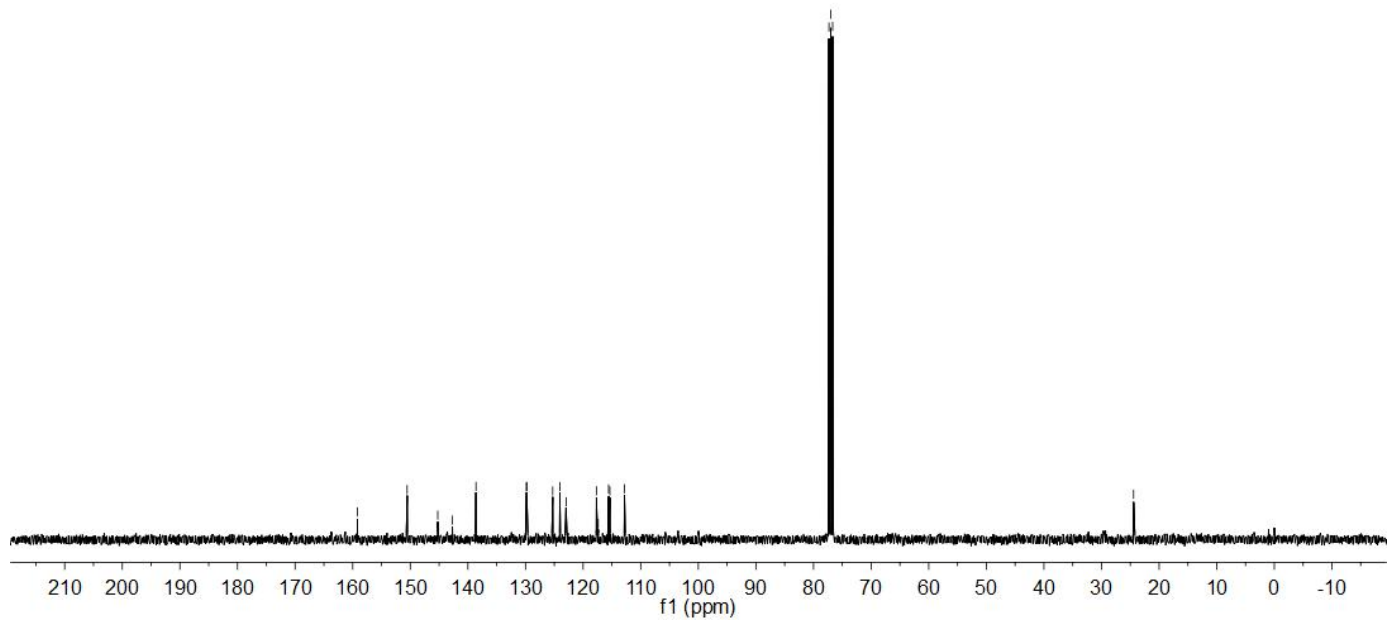


Figure S125. The ${ }^{1} \mathrm{H}$ NMR spectrums of

2-(4-fluorophenyl)-3-(thiophen-2-yl)imidazo[1,2-a]pyridine (5iv)

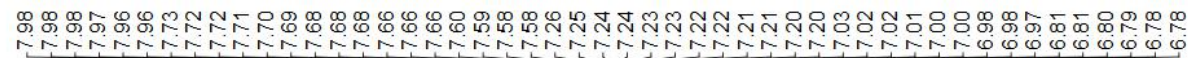
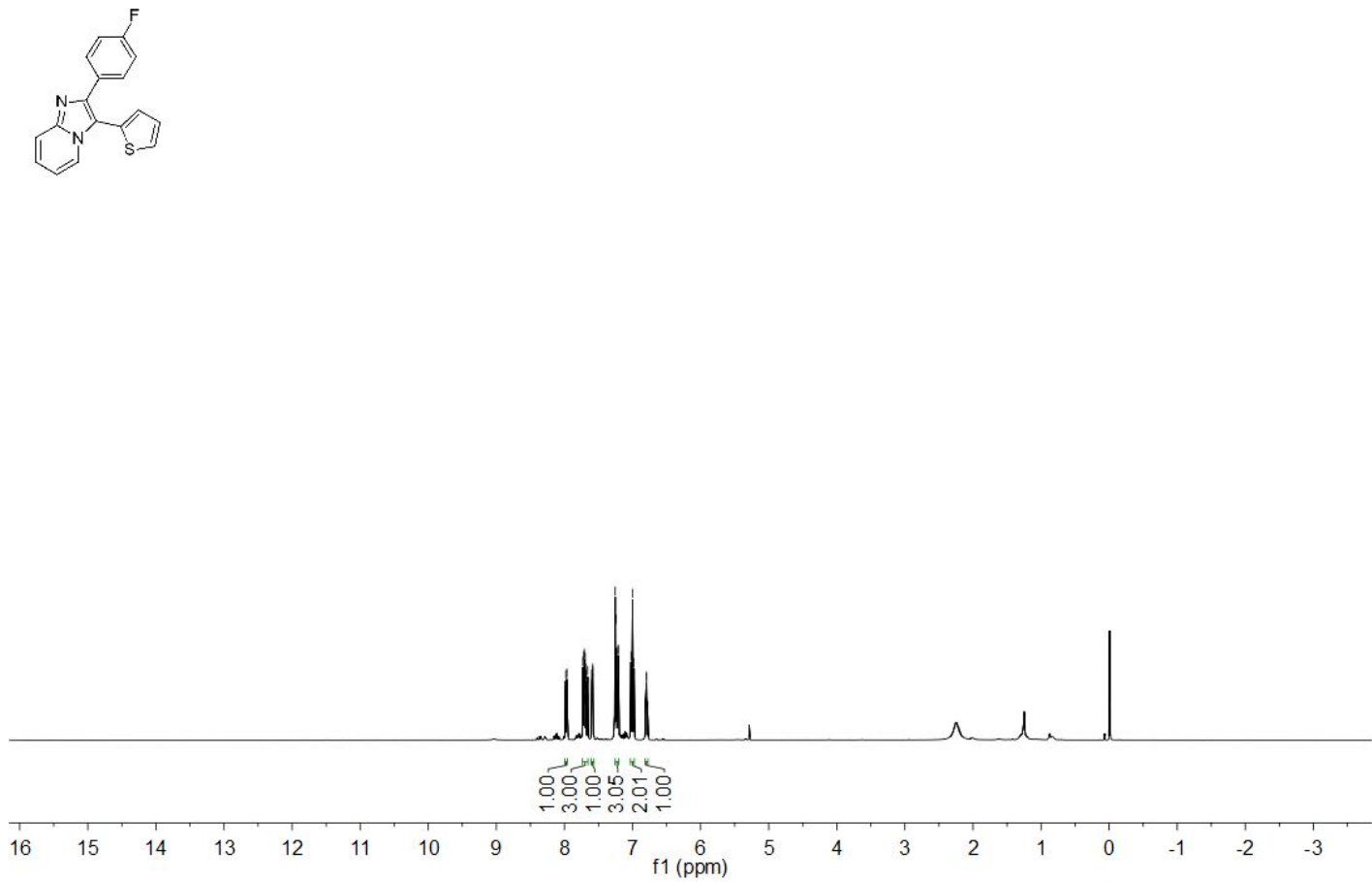

Figure S126. The ${ }^{13} \mathrm{C}$ NMR spectrums of

2-(4-fluorophenyl)-3-(thiophen-2-yl)imidazo[1,2-a]pyridine (5iv)

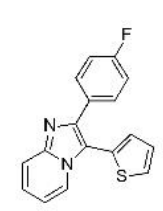

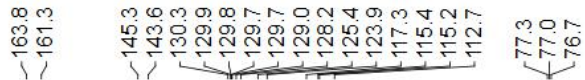

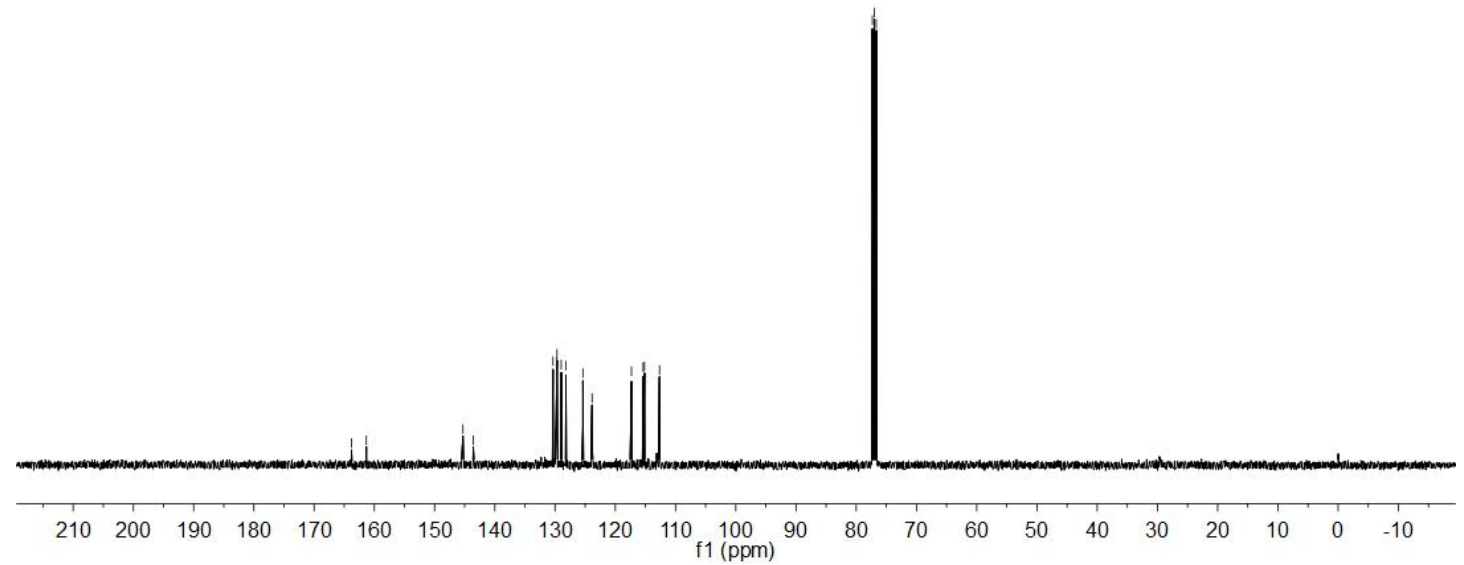


Figure

S127.

The

${ }^{1} \mathbf{H}$

NMR

spectrums

of

3-(6-chloropyridin-3-yl)-2-(4-fluorophenyl)-6-methylimidazo[1,2-a]pyridine(5jp)

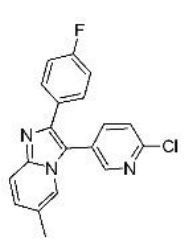

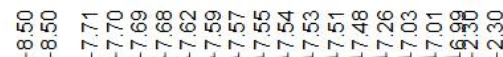

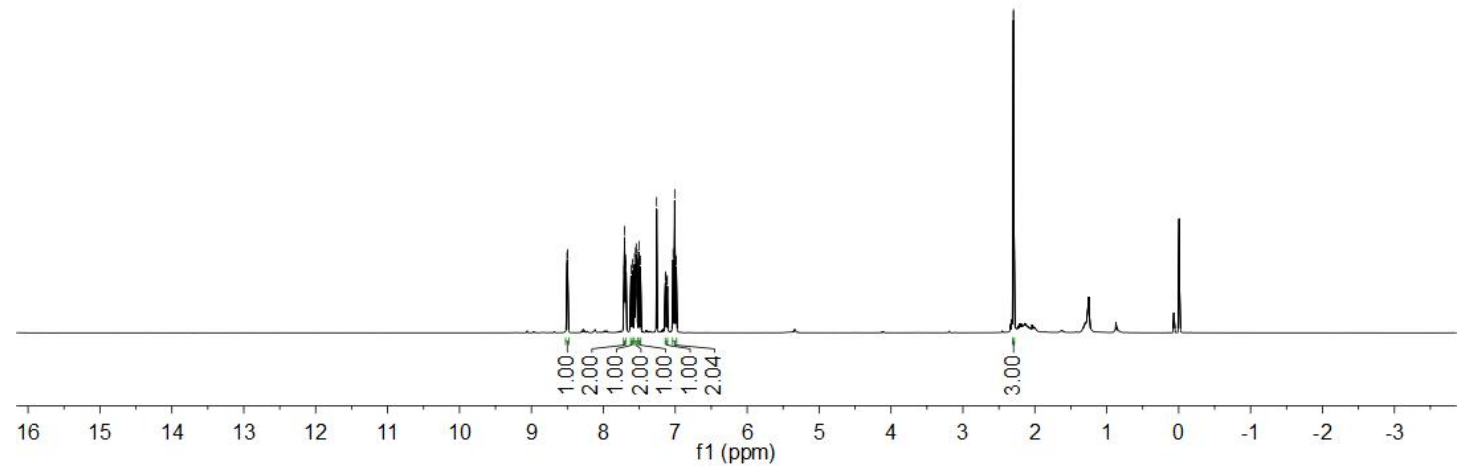

Figure S128. The ${ }^{13} \mathrm{C}$ NMR spectrums of

3-(6-chloropyridin-3-yl)-2-(4-fluorophenyl)-6-methylimidazo[1,2-a]pyridine(5jp)

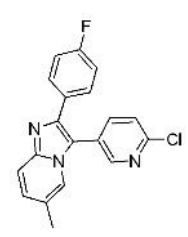

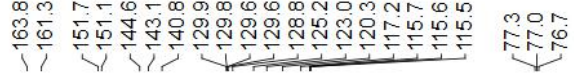

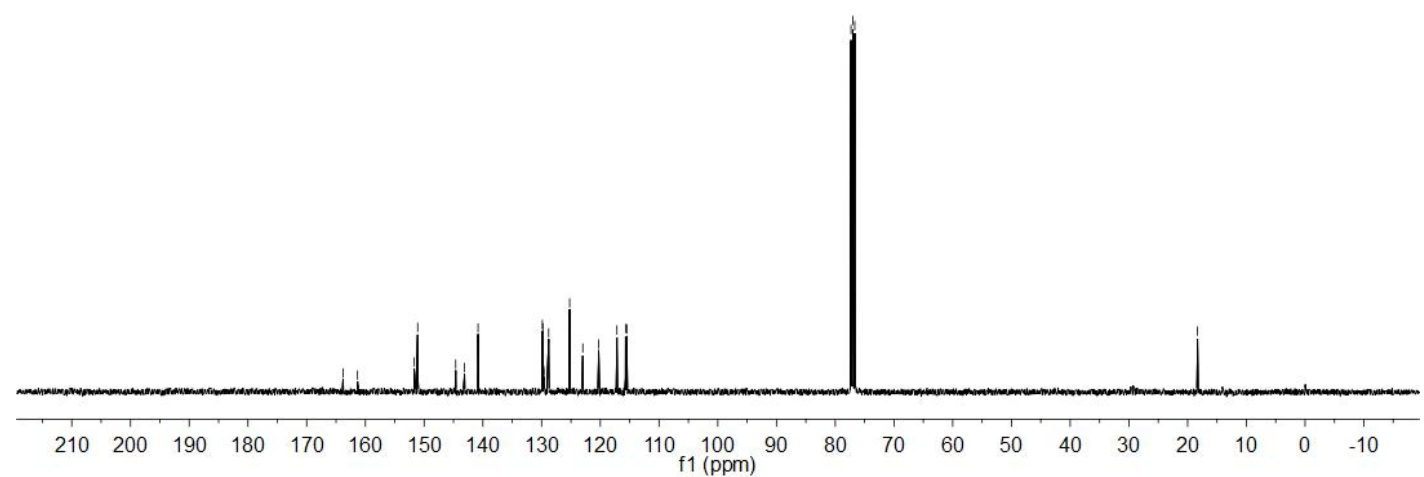


Figure S129. The ${ }^{1} \mathbf{H}$ NMR spectrums of

2-(4-fluorophenyl)-7-methyl-3-(pyrimidin-5-yl)imidazo[1,2-a]pyridine (5kq)

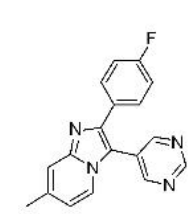

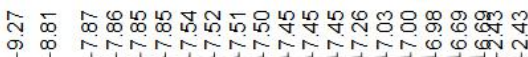

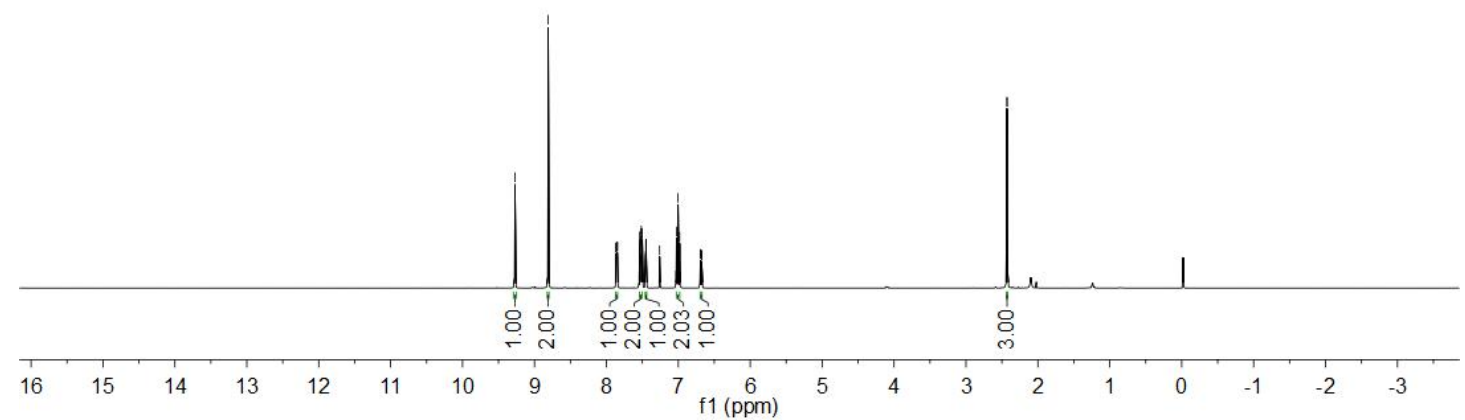

Figure S130. The ${ }^{13} \mathrm{C}$ NMR spectrums of

2-(4-fluorophenyl)-7-methyl-3-(pyrimidin-5-yl)imidazo[1,2-a]pyridine (5kq)

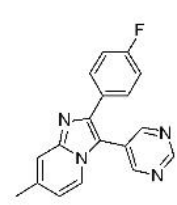

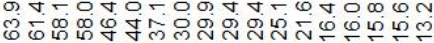

0

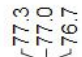

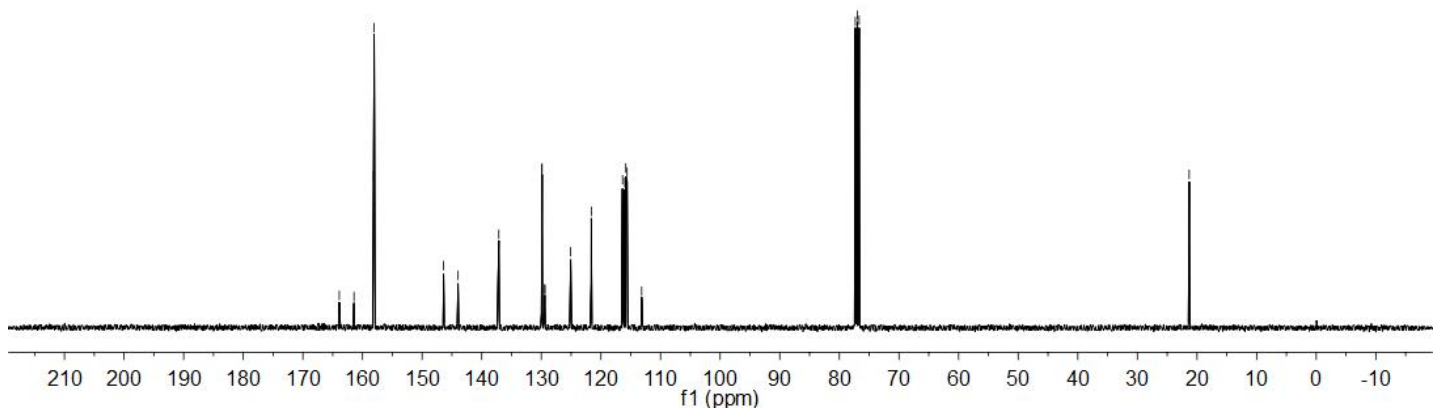


Figure

S131.

The

${ }^{1} \mathbf{H}$

NMRspectrums

of

3-(6-fluoropyridin-3-yl)-6-methyl-2-phenylimidazo[1,2-a]pyridine (5lh)

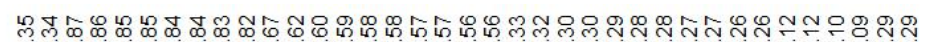

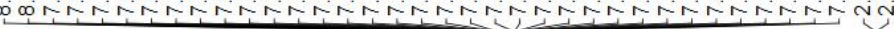
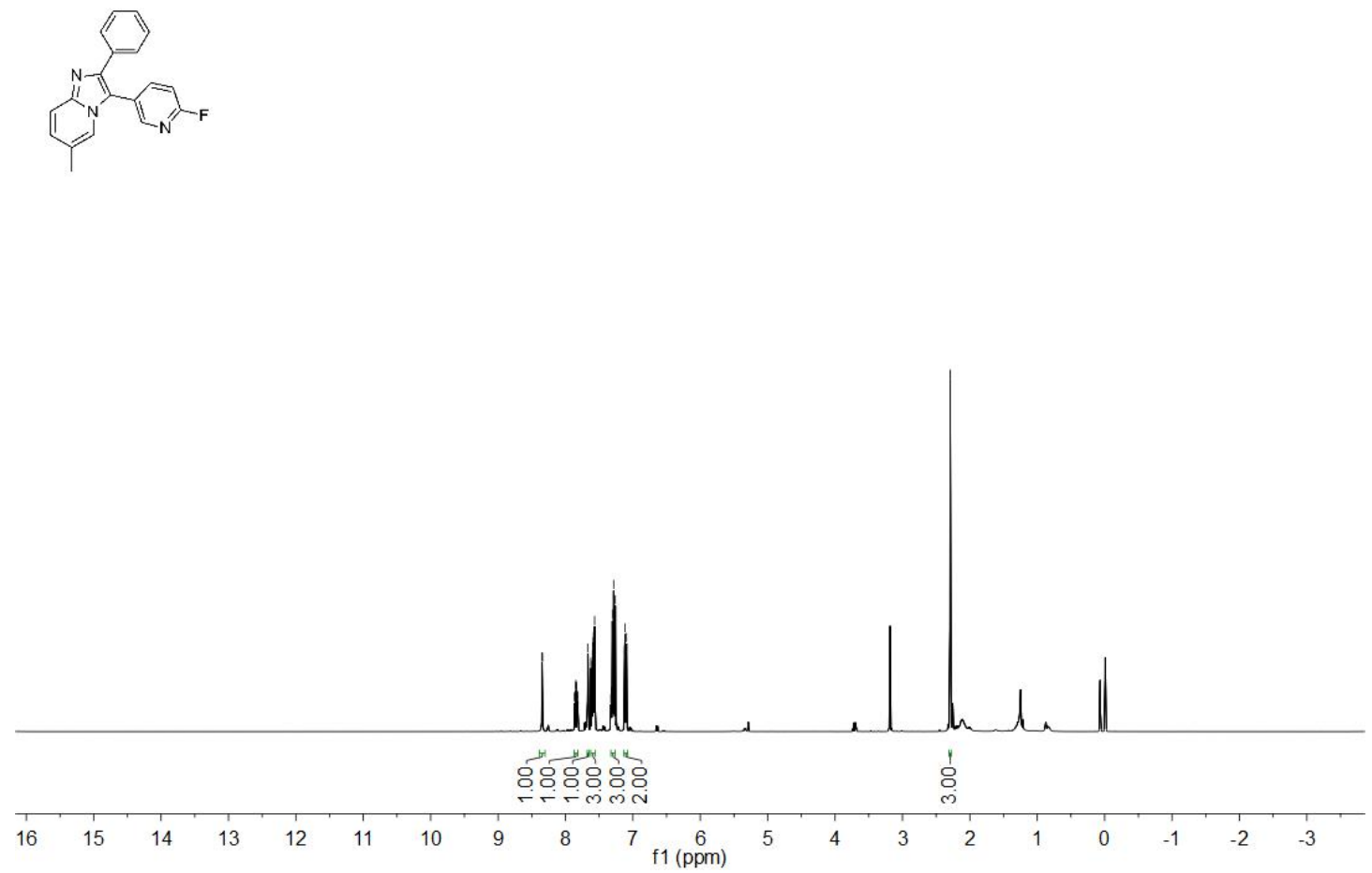

Figure S132. The ${ }^{13} \mathrm{C}$ NMR spectrums of

3-(6-fluoropyridin-3-yl)-6-methyl-2-phenylimidazo[1,2-a]pyridine (5lh)

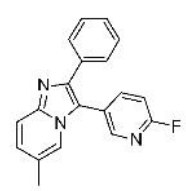

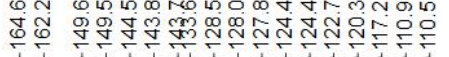
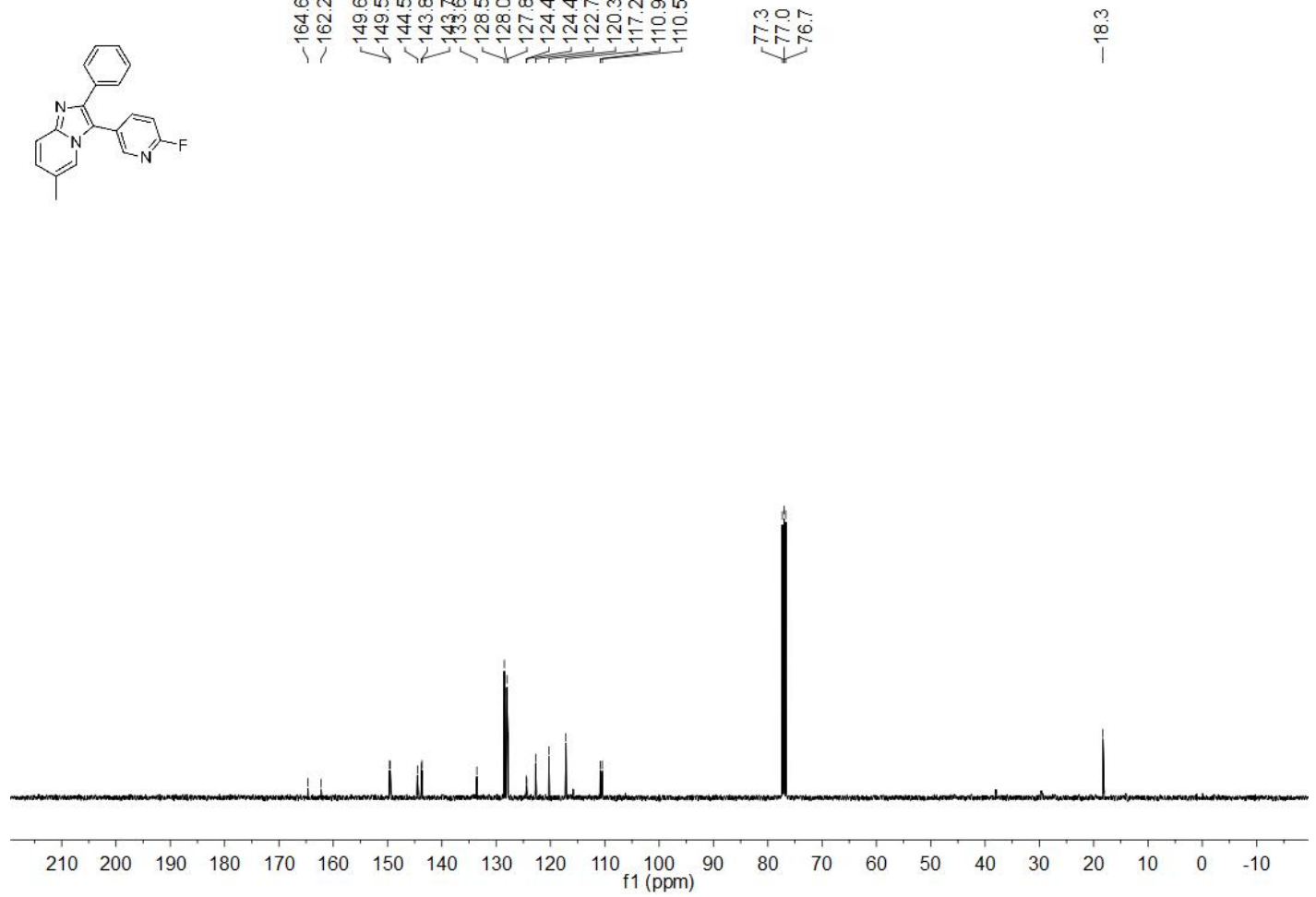
Figure S133. The ${ }^{1} \mathbf{H}$ NMR spectrums of

2-(4-fluorophenyl)-6-methyl-3-(pyrimidin-5-yl)imidazo[1,2-a]pyridine (5lq)
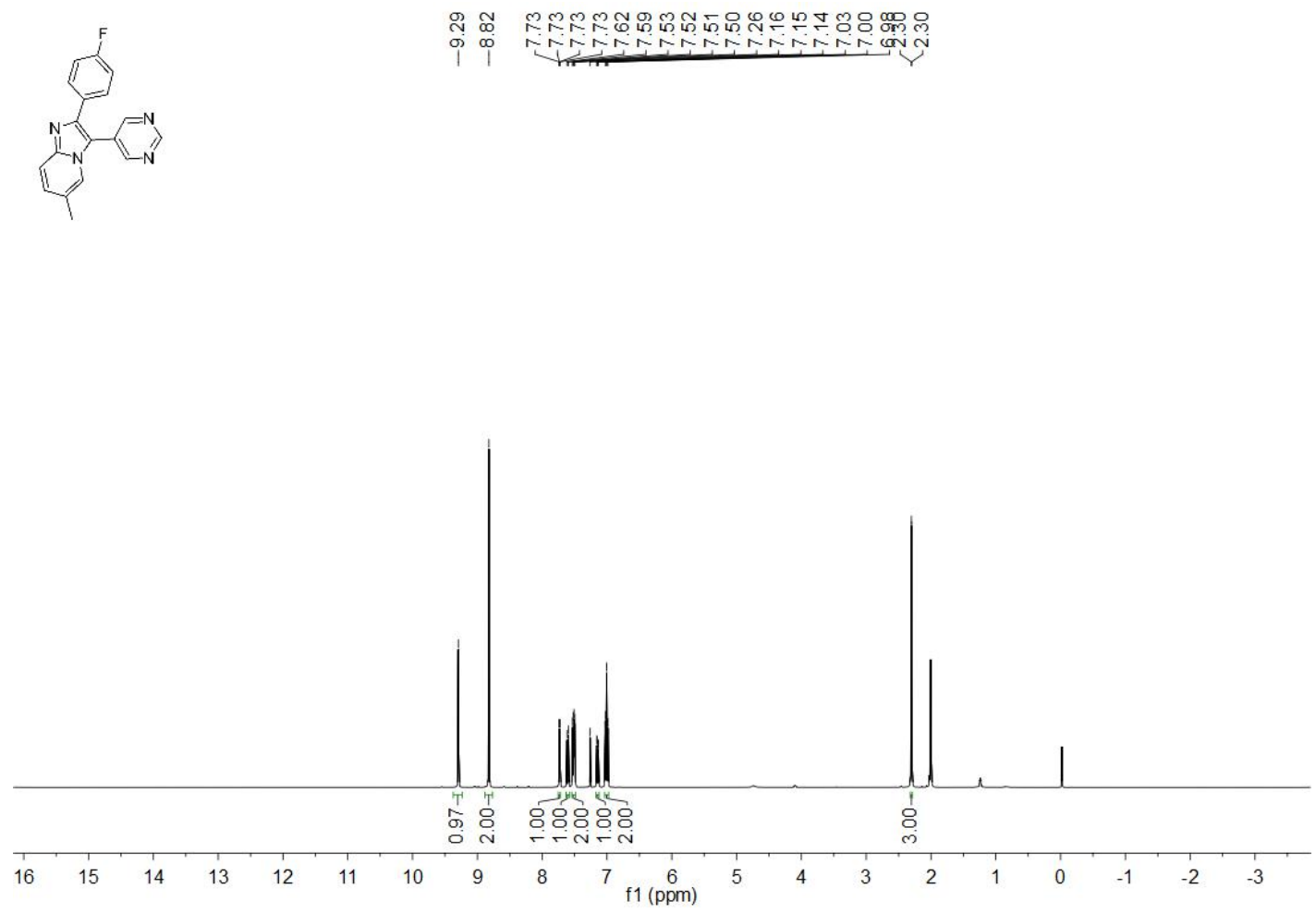

Figure S134. The ${ }^{13} \mathrm{C}$ NMR spectrums of

2-(4-fluorophenyl)-6-methyl-3-(pyrimidin-5-yl)imidazo[1,2-a]pyridine (5lq)
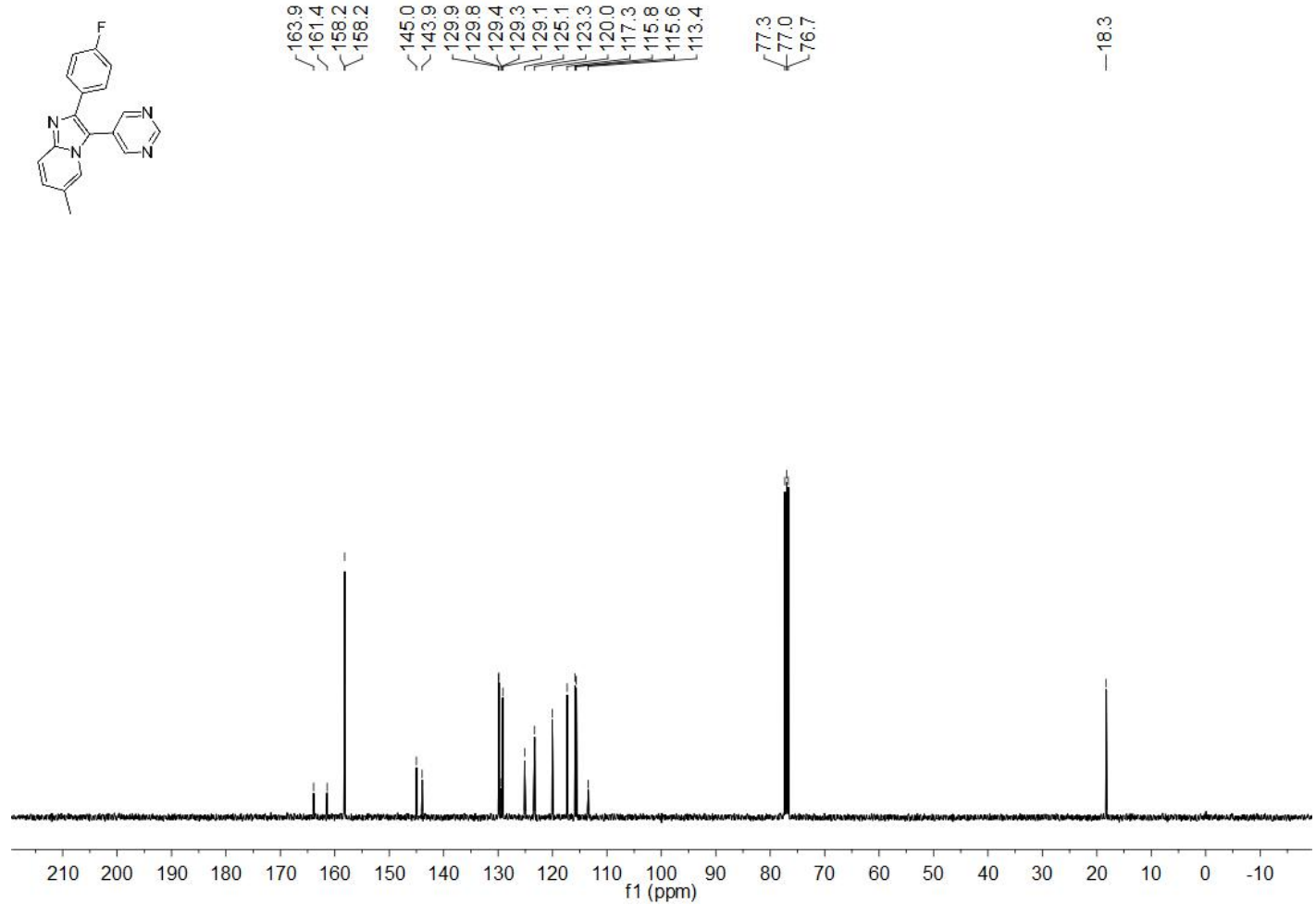
Figure S135. The ${ }^{1} \mathrm{H}$ NMR spectrums of 2-methyl-5-(1-methyl-4-phenyl-1H-pyrazol-5-yl)pyridine (7)
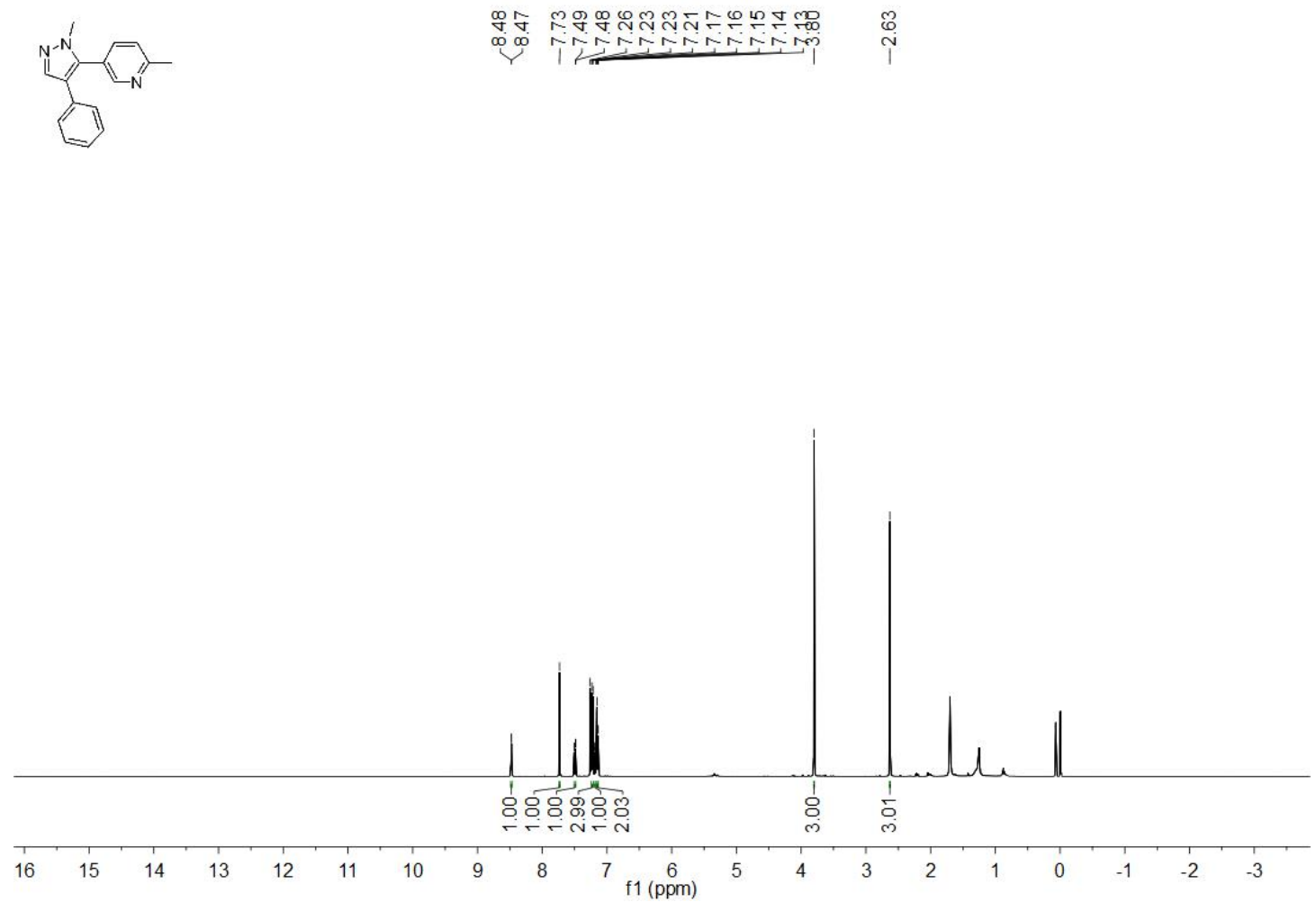

Figure S136. The ${ }^{13} \mathrm{C}$ NMR spectrums of 2-methyl-5-(1-methyl-4-phenyl-1H-pyrazol-5-yl)pyridine (7)

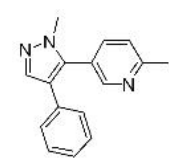

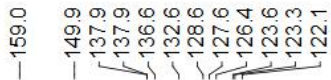

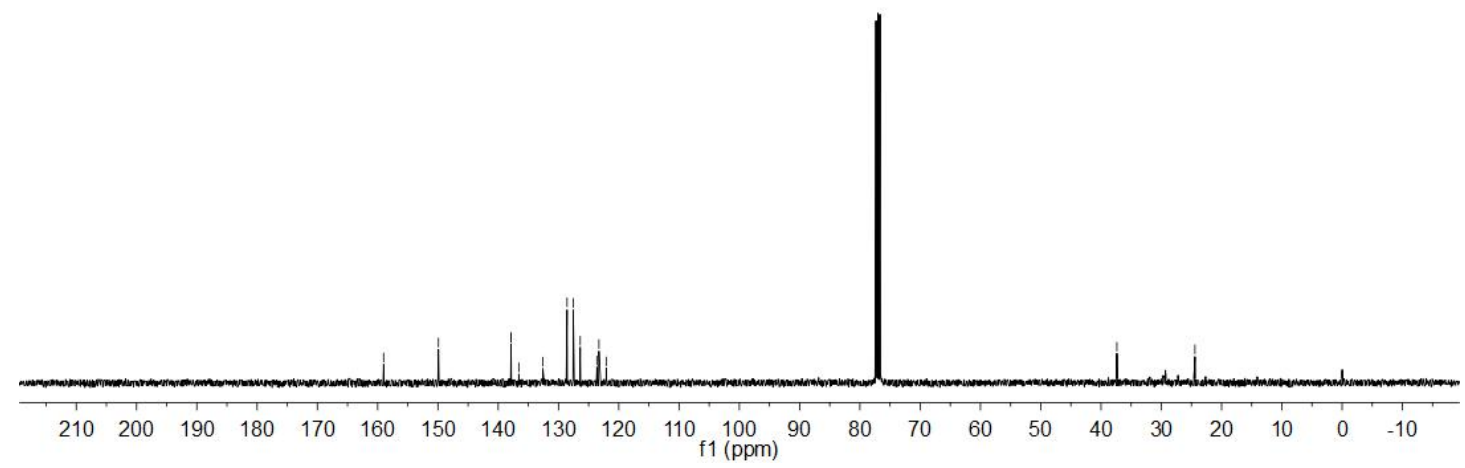


Figure S137. The ${ }^{1}$ H NMR spectrums of Dantrolene
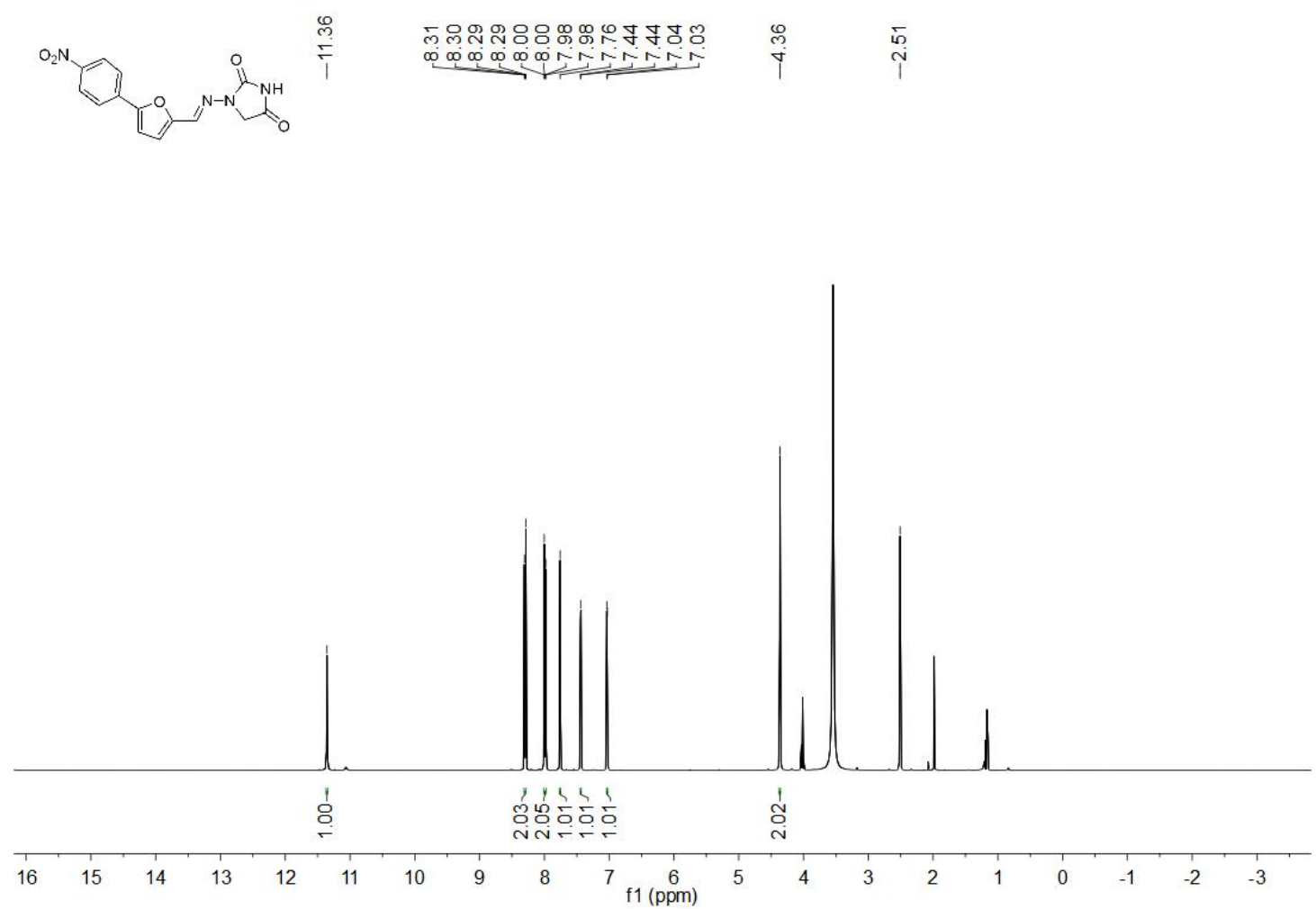

Figure S138. The ${ }^{13} \mathrm{C}$ NMR spectrums of Dantrolene

$$
\text { l }
$$<smiles>O=C1CN(/N=C/c2ccc(-c3ccc([N+](=O)[O-])cc3)o2)C(=O)N1</smiles>

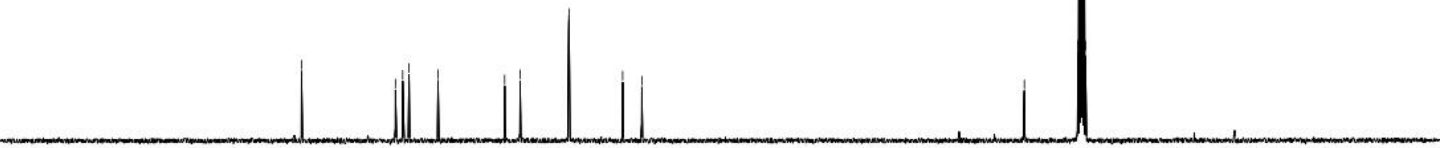

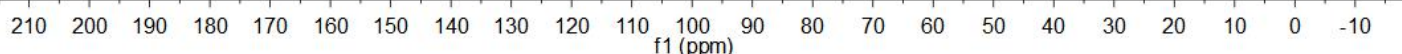


Figure S139. The ${ }^{1} \mathrm{H}$ NMR spectrum of mesopolymers
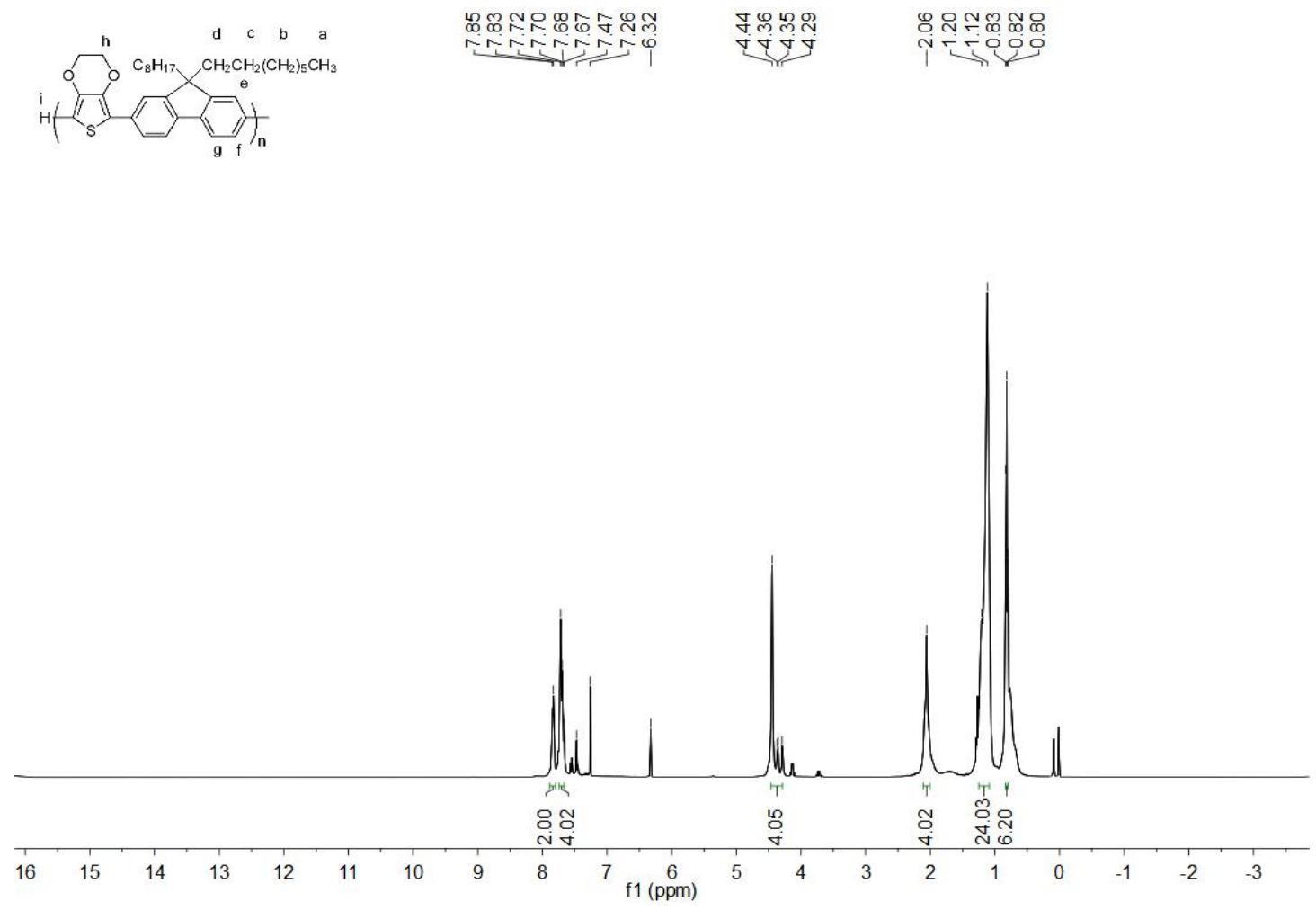

${ }^{1} \mathrm{H}$ NMR (400 MHz, $\left.\mathrm{CDCl}_{3}\right) \delta 7.85-7.83(2 \mathrm{H}), 7.72-7.67(4 \mathrm{H}), 4.44(4 \mathrm{H}), 2.06(4 \mathrm{H}), 1.20-1.12$ $(24 \mathrm{H}), 0.83-0.80(6 \mathrm{H})$.

Figure S140. GPC curves of mesopolymers

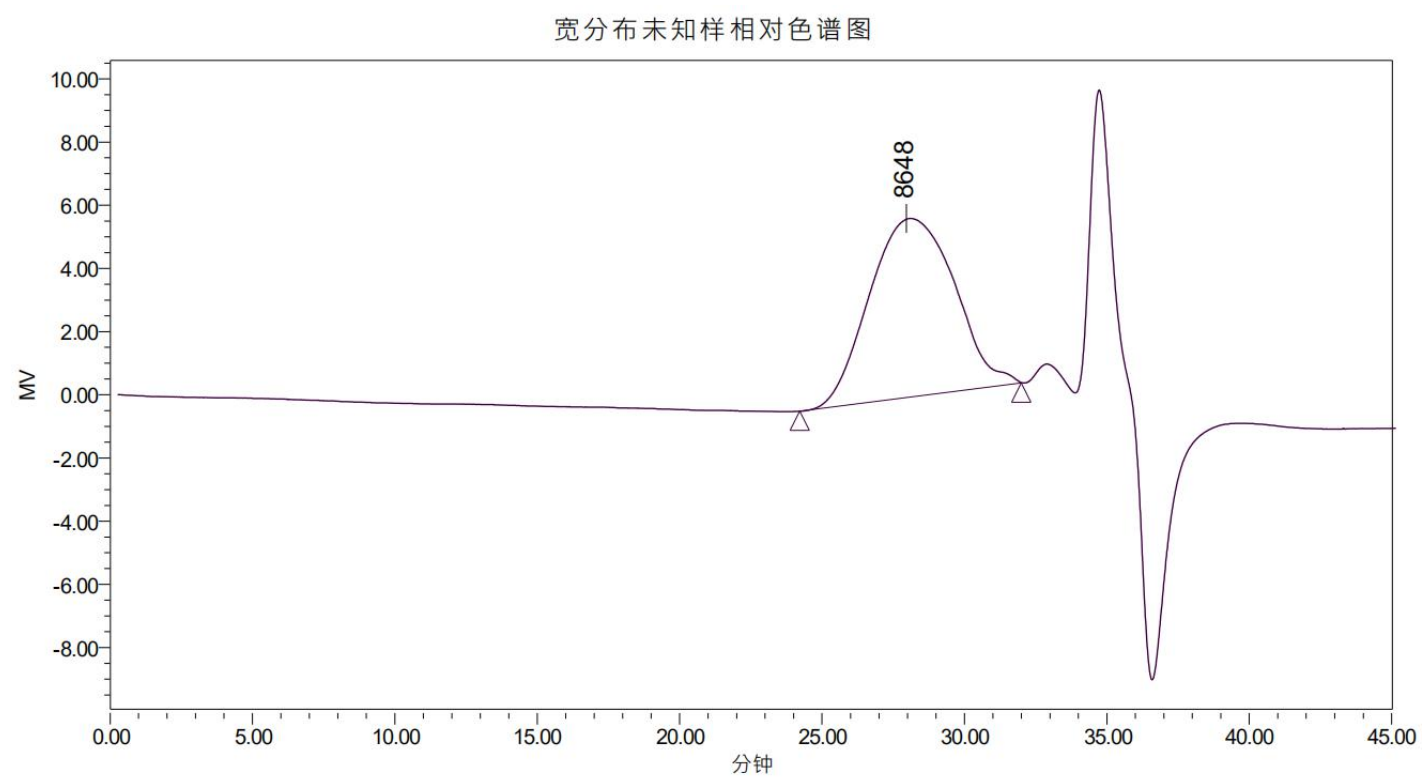

\begin{tabular}{|l|r|r|r|r|r|r|r|r|r|}
\hline & 宽分布未知样相对峰表 \\
\hline 1 & & $\begin{array}{c}M n \\
\text { (道尔顿) }\end{array}$ & $\begin{array}{c}M w \\
\text { (道尔顿) }\end{array}$ & $M P$ & $\begin{array}{c}M z \\
\text { (道尔顿) }\end{array}$ & $\begin{array}{c}M z+1 \\
\text { (道尔顿) }\end{array}$ & 多分散性 & $M z / M w$ & $M z+1 / M w$ \\
\hline
\end{tabular}


Table S1. Pd(OAc)2 catalyzed the direct arylation reaction ${ }^{a, b}$

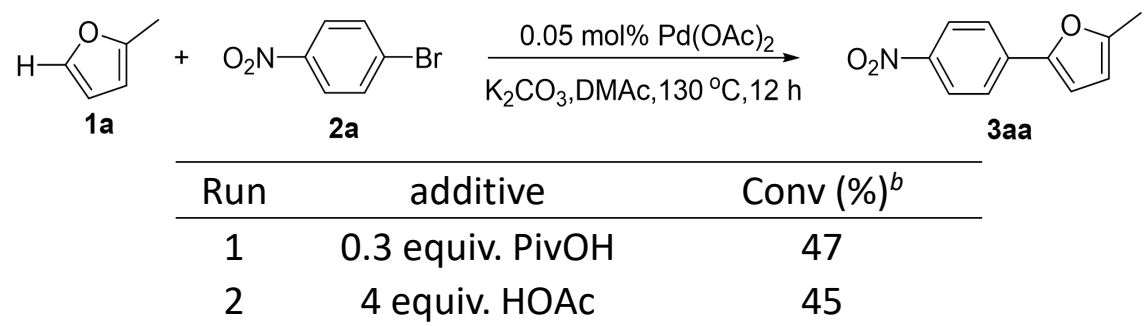

${ }^{a}$ Regents and conditions: 1-Bromo-4-nitrobenzene (1 mmol), 2-methylfuran (3 equiv.), $\mathrm{Pd}(\mathrm{OAc})_{2}(0.05 \mathrm{~mol} \%)$, base: $\mathrm{K}_{2} \mathrm{CO}_{3}$ (2 equiv.), solvent: DMAc $(3 \mathrm{~mL}), 130^{\circ} \mathrm{C}, 12 \mathrm{~h}$, under aerobic conditions. ${ }^{b}$ Isolated yields.

Table S2. Screening of acid additives for the direct arylation reaction $^{a, b}$

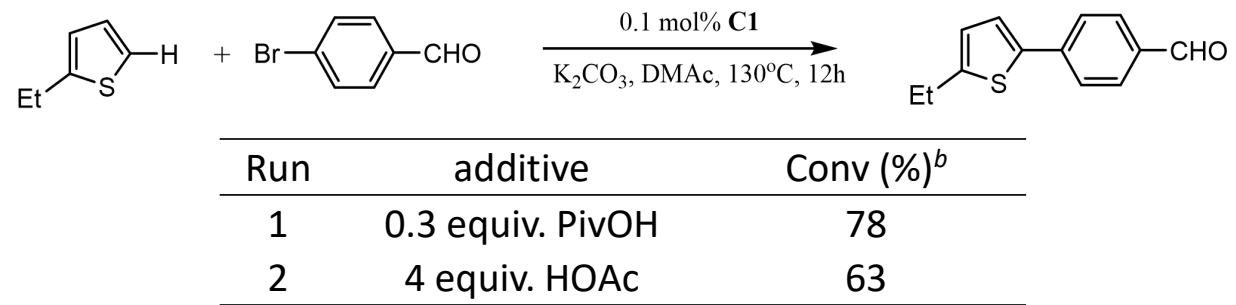

${ }^{a}$ Regents and conditions: 4-bromobenzaldehyde $(1 \mathrm{mmol})$, 2-ethylthiophene (3 equiv.), palladium source $(0.1$ mol\%), base: $\mathrm{K}_{2} \mathrm{CO}_{3}$ (2 equiv.), solvent: DMAc $(3 \mathrm{~mL}), 130{ }^{\circ} \mathrm{C}, 12 \mathrm{~h}$, under aerobic conditions. ${ }^{b}$ Isolated yields.

\section{Table S3. Direct (hetero)arylation of (hetero)arenes with (hetero)aryl chlorides catalyzed by $\mathrm{C} 1$}

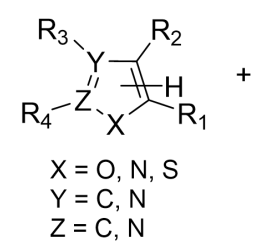

4<smiles>Cc1ccc(-c2ccc(C#N)cc2)o1</smiles>

$3 a b: 16 \%$

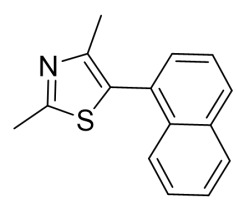

5bm: $<5 \%$

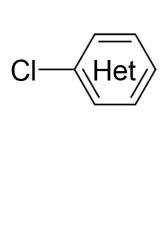

2

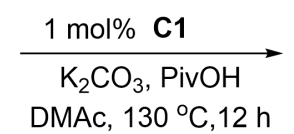

DMAc, $130{ }^{\circ} \mathrm{C}, 12 \mathrm{~h}$

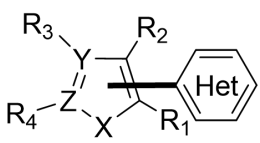

$\mathrm{X}=\mathrm{O}, \mathrm{N}, \mathrm{S}$

$\mathrm{Y}=\mathrm{C}, \mathrm{N}$
$\mathrm{Z}=\mathrm{C}, \mathrm{N}$

5

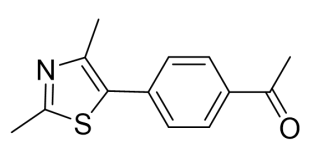

5bd: $8 \%$<smiles>Cn1ncc(Br)c1-c1ccc(C#N)cc1</smiles>

$5 \mathrm{fb}:<5 \%$

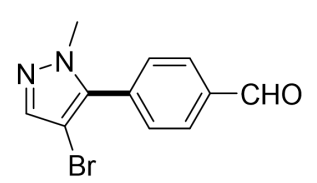

5fc: $31 \%$

Reaction conditions: (hetero)aryl chlorides ( $1 \mathrm{mmol}$ ), heteroaryl (3 equiv.), $\mathbf{C 1}(1 \mathrm{~mol} \%), \mathrm{K}_{2} \mathrm{CO}_{3}$ ( 2 equiv.), DMAc ( $3 \mathrm{~mL}$ ), PivOH (30 mol\%), $130{ }^{\circ} \mathrm{C}, 12 \mathrm{~h}$, in air. Isolated yields. 